\title{
Stochastic Perron for Stochastic Target Problems
}

\author{
by \\ Jiaqi Li \\ A dissertation submitted in partial fulfillment \\ of the requirements for the degree of \\ Doctor of Philosophy \\ (Mathematics) \\ in The University of Michigan \\ 2016
}

Doctoral Committee:

Professor Erhan Bayraktar, Co-Chair

Professor Uday Rajan, Co-Chair

Professor Joseph G. Conlon

Associate Professor Johannes Muhle-Karbe

Assistant Professor Sergey Nadtochiy

Professor Virginia R. Young 
Jiaqi Li 2016

All Rights Reserved 
To my wife, mom and dad 


\section{ACKNOWLEDGEMENTS}

This thesis would not have been possible without the enormous support and guidance from my Ph.D. advisor Professor Erhan Bayraktar. From him, not only I learnt mathematics, but also the right way to approach research and how to write and present. I also thank him for providing many opportunities on my professional growth, for his patience and support and for many other things and beyond.

I would like to thank Professors Uday Rajan, Joseph Conlon, Johannes MuhleKarbe, Sergey Nadtochiy and Virginia Young, for serving on my dissertation committee and reading my thesis carefully. In particular, I am deeply grateful to my co-advisor Professor Uday Rajan for providing me with research freedom, for his guidance when necessary and for his effort and time in reading and correcting my thesis carefully.

I owe many thanks to the Department of Mathematics, University of Michigan for providing me an academic home and the financial support during last five years.

I want to thank Zhou Zhou and Yuchong Zhang for discussing research problems and sharing their graduate study experiences, and Alexander Munk for his help in revising my paper, which resulted in much improvement of the work. I also thank Xueying Hu for her advice and help in my future career.

Furthermore, I would like to express my appreciation to Weichen Gu, Wei Li, Bowei Wu, Feng Wei, Yitong Sun, Yiwei Zhang, Yingchuan Wang, Yebin Tao and many others who have have been by my side encouraging me during difficult times, 
and who have also been sharing fun and joy in life in the past five years.

I cannot finish without saying how grateful I am to my family: my mother Yaping Qi, my father Baoliang Li and most importantly, my wife Jingjing Ma. Their unconditional love and support have made me who I am today. To them I dedicate this thesis. 


\section{TABLE OF CONTENTS}

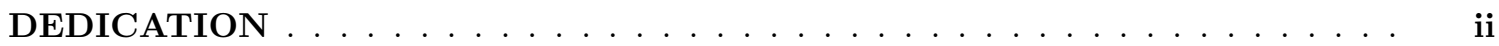

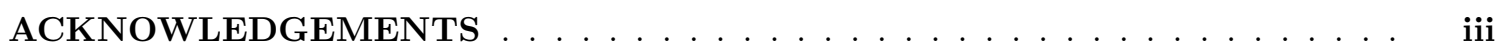

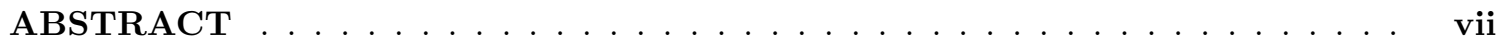

\section{CHAPTER}

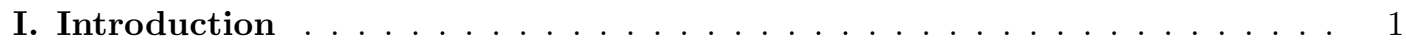

II. Stochastic Perron for Stochastic Target Problems in a Jump Diffusion

Model . . . . . . . . . . . . . . . . . . . . . 6

2.1 Outline of this chapter $\ldots \ldots \ldots \ldots \ldots \ldots$

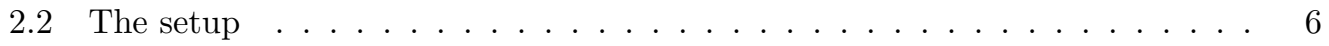

2.2.1 The Hamilton-Jacobi-Bellman equation $\ldots \ldots \ldots \ldots$

2.2 .2 Stochastic solutions . . . . . . . . . . . . . . . . 10

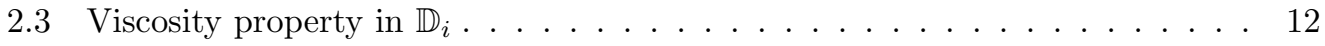

2.4 Boundary conditions . . . . . . . . . . . . . . . . . . . . . 22

2.5 Verification by comparison . . . . . . . . . . . . . . . . 29

2.6 Stochastic control as a stochastic target problem . . . . . . . . . . . . . 33

2.7 Appendix: the nonemptiness of $\mathbb{U}^{+}$and $\mathbb{U}^{-} \ldots \ldots \ldots \ldots$. . . . . . . 37

III. Stochastic Perron for Stochastic Target Problems with a Stopper in a Jump Diffusion Model . . . . . . . . . . . . . . . . . . . . . . . . . . . . 42

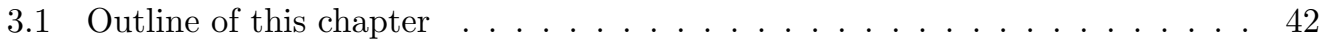

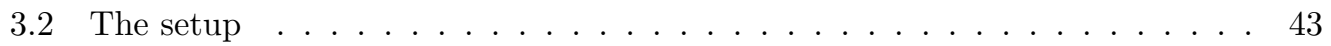

3.2.1 The Hamilton-Jacobi-Bellman equation _. . . . . . . . . . . . 46

3.3 The stochastic target problem with a non-cooperative stopper . . . . . . . 47

3.3.1 Viscosity property in $\mathbb{D}_{i} \ldots \ldots \ldots \ldots \ldots$. . . . . . . . 48

3.3 .2 Boundary conditions . . . . . . . . . . . . . . . . 55

3.3 .3 Verification by comparison . . . . . . . . . . . . . . . 60

3.3.4 Stochastic controller-stopper game as a stochastic target problem with a non-cooperative stopper . . . . . . . . . . . . 62

3.4 The stochastic target problem with a cooperative stopper . . . . . . . . . 66

3.4 .1 Viscosity property in $\mathbb{D}_{i} \ldots \ldots \ldots \ldots$. . . . . . . 67

3.4 .2 Boundary conditions . . . . . . . . . . . . . . . 77

3.4 .3 Verification by comparison . . . . . . . . . . . . . . 79

3.4.4 Stochastic cooperative controller-stopper problem as a stochastic target problem with a cooperative stopper . . . . . . . . . . 81

3.5 Appendix . . . . . . . . . . . . . . . . . . . 84

3.5.1 The nonemptiness of $\mathbb{U}_{\text {unco }}^{+}, \mathbb{U}_{\text {unco }}^{-}, \mathbb{U}_{\text {co }}^{+}$and $\mathbb{U}_{\text {co }}^{-} \ldots \ldots \ldots . . .84$ 
3.5.2 The equivalence results . . . . . . . . . . . . . . . . 88

IV. Stochastic Perron for Stochastic Target Games . . . . . . . . . . . . . . . . 91

4.1 Outline of this chapter . . . . . . . . . . . . . . . . . . 91

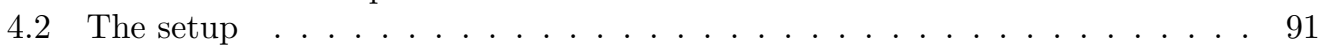

4.2 .1 The value function . . . . . . . . . . . . . . . . 91

4.2.2 The Hamilton-Jacobi-Bellman equation . . . . . . . . . . . . . . 95

4.2.3 Stochastic solutions . . . . . . . . . . . . . . . . . 95

4.2.4 Additional technical assumptions. . . . . . . . . . . . . . . . . 97

4.2.5 The nonemptiness of $\mathbb{U}^{+}$and $\mathbb{U}^{-} \ldots \ldots \ldots \ldots$

4.3 The main results . . . . . . . . . . . . . . . . . . . . . . . . . 99

4.4 Appendix . . . . . . . . . . . . . . . . . . . . . . 120

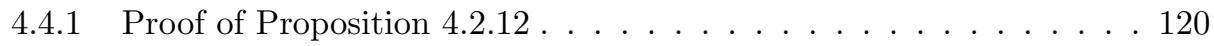

4.4 .2 Proof of Proposition 4.2.17 . . . . . . . . . . . . . . 122

4.4.3 Some well-known results from the theory of viscosity solutions . . . 124

V. Stochastic Perron for Stochastic Target Games with a Stopper . . . . . . 126

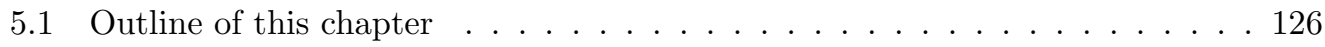

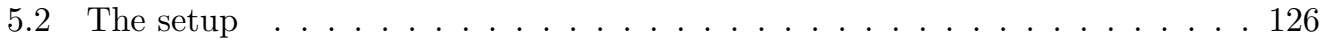

5.3 The super-hedging problem . . . . . . . . . . . . . . . . . 131

5.4 The sub-hedging problem . . . . . . . . . . . . . . . . 138

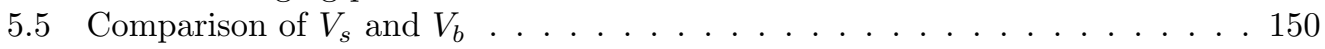

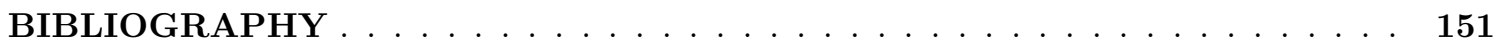




\begin{abstract}
Stochastic Perron for Stochastic Target Problems

by

Jiaqi Li
\end{abstract}

Chairs: Erhan Bayraktar and Uday Rajan

This thesis is devoted to the application of stochastic Perron's method in stochastic target problems. In Chapters II-V, we study different stochastic target problems in various setup. For each target problem, stochastic Perron's method produces a viscosity sub-solution and super-solution to its associated Hamilton-Jacobi-Bellman (HJB) equation. We then characterize the value function in each problem as the unique viscosity solution to the associated HJB equation using a comparison result.

In Chapter II, we investigate stochastic target problems in a jump diffusion setup, where the controls are unbounded. Since classical control problems can be analyzed under the framework of stochastic target problems, we use our results to generalize the results of Bayraktar and Sîrbu (SIAM J Control Optim 51(6): 4274-4294, 2013) to problems with controlled jumps.

In Chapter III, we study stochastic target problems with a stopper under the setup as in Chapter II. We prove that the target problem with a cooperative stopper (resp. with a non-cooperative stopper) can be expressed in terms of a cooperative controller-stopper problem (resp. a controller-stopper game).

In Chapter IV, we analyze the framework of stochastic target games, in which one 
player tries to find a strategy such that the state processes reach a given target at a deterministic time no matter which action is chosen by the other player (Nature). Besides obtaining the PDE characterization of the value function, we also prove the dynamic programming principle as a corollary.

In Chapter V, we study two types of stochastic target games with a stopper under the framework of Chapter IV. We show that the value function in each problem is the unique viscosity solution of a variational HJB equation. We also compare the value functions and prove that they coincide when the control set of Nature is a singleton. 


\section{CHAPTER I}

\section{Introduction}

Introduced by the seminal papers [31], [32] and [33], the stochastic target problem is a new type of optimal control problem. Unlike in the usual stochastic control problem, the goal in a stochastic target problem is to drive a controlled process to a given target at a pre-specified time almost surely by choosing an appropriate admissible control. Thus, these problems are viewed as generalizations of the superhedging problems in mathematical finance. Later, the previous work in $[31,32,33]$ are generalized in $[12,25]$ (to jump diffusions), [14] (to unbounded controls), [15] (to stochastic target games with controlled loss) and [16] (to stochastic target games with almost sure target). These papers provide a characterization of the associated value function as a viscosity solution to a non-linear HJB equation using dynamic programming principle. However, the rigorous proof of the dynamic programming principle is often difficult and contains subtle technical issues.

In this thesis, we will investigate stochastic target problems with various setup using a new methodology, namely stochastic Perron's method. This method was first introduced in [8] for analyzing linear problems, in [10] for Dynkin games involving free-boundary games, and in [9] for stochastic control problems. More recently, it was adjusted to solve exit time problems in [28], state constraint problems in [27], 
singular control problems in [2], stochastic games in [30], and control problems with model uncertainty in [29] and [3].

Stochastic Perron's method is a verification approach in that it does not use the dynamic programming principle to show that the value function is a viscosity solution. The main difficulty of this approach as well as the conceptual contribution is to construct two classes of functions that envelope the value function and that are stable under pairwise minimization and maximization, respectively. Once this is established, the technical contribution is to demonstrate that the supremum over the first class is a lower semi-continuous (LSC) viscosity super-solution and the infimum over the second class (the functions larger than the value function) is an upper semi-continuous (USC) viscosity sub-solution. Assuming that a comparison principle holds, we show that the infimum over the second class and the supremum over the first class (which sandwich the value function) are equal, and hence, the value function is the unique viscosity solution. Since we only work with the envelopes, not the value function itself, we never use the dynamic programming principle. Our result can be seen as an elementary alternative based only on Itô's Lemma and the comparison principle, which also has to be proved to identify the value function as the unique viscosity solution of the HJB partial differential equation.

In each of Chapters II-IV, stochastic Perron's method produces a viscosity subsolution and super-solution of an HJB equation. The value function is then characterized as the unique viscosity solution to the associated HJB equation using a comparison result.

In Chapter II, we consider a stochastic target problem with a general stochastic target setup from [25]. Our controls are unbounded and the controlled processes are jump diffusions. The main reason for using unbounded controls is that we are able 
convert an ordinary control problem into a stochastic target problem with unbounded admissible controls, using the embedding result of [13]. With such a result, we generalize [9] to the setting of controlled jumps.

We also generalize our earlier result in $[6]$ in the sense that we consider unbounded controls and controlled jumps. The presence of the jumps and the unbounded control set brings new technical difficulties: in contrast to [6], the relaxed semi-limits are introduced for the PDE characterization, which have a nontrivial impact on the formulation of the associated PDEs and the derivation of viscosity properties of the value function using stochastic Perron's method, especially at the boundary. Of particular importance is the relaxation with respect to the test function, which appears because we consider jumps. This chapter is based on [7].

In Chapter III, we study two types of stochastic target problems with a stopper in the jump diffusion model as presented in Chapter II. One type of the target problems involves a cooperative stopper (Section 3.3), while the other involves a noncooperative stopper, which might play against the controller in a non-anticipative way (Section 3.4). Besides the PDE characterization, another major contribution in this chapter is the we are able to establish the "equivalence" between a cooperative controller-stopper problem (resp. a controller-stopper game) and the stochastic target problem with a cooperative stopper (resp. with a non-cooperative stopper) introduced in this chapter. We show that

1. The HJB equations associated to a (semi) controller-stopper game can be deduced from a stochastic target problem with a non-cooperative stopper.

2. Any cooperative controller-stopper problem admits a natural representation in terms of a stochastic target problem with a cooperative stopper.

Such equivalence results, along with the analysis in stochastic target problems in 
this chapter, provide us with an alternative to solve the cooperative controllerstopper problem and controller-stopper game. For the discussion about the cooperative controller-stopper problem and controller-stopper game, we refer the readers to $[19,21]$ and $[22,23,24,17,11]$. This chapter is based on a working paper by Bayraktar and Li.

In Chapter IV, we will analyze a stochastic game where a controller tries to find a strategy such that the controlled state process almost-surely reaches a given target at a pre-specified time, no matter which control is chosen by an adverse player (Nature). More precisely, the controller has access to a filtration generated by a Brownian motion and can observe and react to nature, who may choose a parametrization of the model to be totally adverse to the controller, in a non-anticipative way. This stochastic target game was introduced and analyzed in [16].

With stochastic Perron's method, we obtain the PDE characterization of the value function without going through the geometric dynamic programming principle first. This enables us to avoid using Krylov's method of shaken coefficients which requires the concavity of the Hamiltonian. This way, we provide a more elementary proof to the results in [16] and obtain dynamic programming principle as a byproduct. This chapter is based on [6]. Parts of the work have been presented at the Financial/Actuarial Mathematics Seminar, University of Michigan, September 3, 2014.

In Chapter V, we study two types of stochastic target games with a stopper. In the first type of the target games, both Nature and the stopper might be totally adverse to the controller in a non-anticipative way. The controller aims to drive the controlled processes to a target no matter what action is chosen by Nature or when the game is stopped. However, in the other type, the controller's goal is to beat the target by applying both a control strategy and a stopping strategy, regardless of 
Nature's action. With such a formulation, Nature's control can be interpreted as a parametrization of the model. Thus, the first problem can be interpreted as superhedging American options with model uncertainty in the context of mathematical finance, while the other can be understood as sub-hedging American options with model uncertainty. Besides obtaining the PDE characterization of the value functions, we also compare the two value functions without proving any duality results and verify that they coincide when the control set of Nature is a singleton. This chapter is based on a working paper by Bayraktar and Li. 


\section{CHAPTER II}

\section{Stochastic Perron for Stochastic Target Problems in a Jump Diffusion Model}

\subsection{Outline of this chapter}

In this chapter, we analyze a stochastic target problem in a general stochastic target setup from [25]. In Section 2.2, the setup of the problem, the related HJB equation and the definitions of the stochastic semi-solutions are first introduced. In Sections 2.3 and 2.4, we prove the viscosity properties in the parabolic interior and at the boundary, respectively. In Section 2.5, we use the comparison principle to close the gap between the viscosity super-solution and sub-solution and demonstrate the uniqueness of the viscosity solution to the associated HJB equation. In Section 2.6, we see how an optimal control problem can be converted into a stochastic target problem. Some technical results are delegated to the appendix (Section 2.7). Our main results are Theorems 2.3.3, 2.4.1, 2.5.5 and 2.6.2.

\subsection{The setup}

Throughout this thesis, the superscript ${ }^{\top}$ stands for transposition, $|\cdot|$ for the Euclidean norm of a vector in $\mathbb{R}^{n}$ and $\|\cdot\|$ for the Frobenius norm of a matrix. For a subset of $\mathcal{O}$ of $\mathbb{R}^{n}$, we denote by $\operatorname{Int}(\mathcal{O})$ its interior. We also denote the open ball of radius $r>0$ centered at $x \in \mathbb{R}^{n}$ by $B_{r}(x)$ and the set of $n \times n$ matrices (resp. 
symmetric matrices) by $\mathbb{M}^{n}$ (resp. $\left.\mathbb{S}^{n}\right)$. Inequalities and inclusion between random variables and random sets, respectively, are in the almost sure sense unless otherwise stated.

Given a complete probability space $(\Omega, \mathcal{F}, \mathbb{P})$, let $\left\{\lambda_{i}(\cdot, d e)\right\}_{i=1}^{I}$ be a collection of independent integer-valued $E$-marked right-continuous point processes defined on this space. Here, $E$ is a Borel subset of $\mathbb{R}$ equipped with the Borel sigma field $\mathcal{E}$. Let $\lambda=\left(\lambda_{1}, \lambda_{2}, \cdots, \lambda_{I}\right)^{\top}$ and $W=\left\{W_{s}\right\}_{0 \leq s \leq T}$ be a $d$-dimensional Brownian motion defined on the same probability space such that $W$ and $\lambda$ are independent. Given $t \in[0, T]$, let $\mathbb{F}^{t}=\left\{\mathcal{F}_{s}^{t}, t \leq s \leq T\right\}$ be $\mathbb{P}$-completed filtration generated by $W$. $-W_{t}$ and $\lambda([0, \cdot], d e)-\lambda([0, t], d e)$. Set $\mathcal{F}_{s}^{t}=\mathcal{F}_{t}^{t}$ for $0 \leq s<t$. We will use $\mathcal{T}_{t}$ to denote the set of $\mathbb{F}^{t}$-stopping times valued in $[t, T]$. Given $\tau \in \mathcal{T}_{t}$, the set of $\mathbb{F}^{t}$-stopping times valued in $[\tau, T]$ will be denoted by $\mathcal{T}_{\tau}$.

Assumption 2.2.1. $\lambda$ satisfies the following:

1. $\lambda(d s, d e)$ has intensity kernel $m(d e) d s$ such that $m_{i}$ is a Borel measure on $(E, \mathcal{E})$ for any $i=1, \cdots, I$ and $\hat{m}(E)<\infty$, where $m=\left(m_{1}, \cdots, m_{I}\right)^{\top}$ and $\hat{m}=$ $\sum_{i=1}^{I} m_{i}$.

2. $E=\operatorname{supp}\left(m_{i}\right)$ for all $i=1,2, \cdots, I$. Here, $\operatorname{supp}\left(m_{i}\right):=\left\{e \in E: e \in N_{e} \in\right.$ $\left.T_{E} \Longrightarrow m_{i}\left(N_{e}\right)>0\right\}$, where $T_{E}$ is the topology on $E$ induced by the Euclidean topology

3. There exists a constant $C>0$ such that

$$
\mathbb{P}(\{\hat{\lambda}(\{s\}, E) \leq C \text { for all } s \in[0, T]\})=1 \text {, where } \hat{\lambda}=\sum_{i=1}^{I} \lambda_{i} \text {. }
$$

The above assumption implies that there are a finite number of jumps during any finite time interval. Let $\tilde{\lambda}(d s, d e):=\lambda(d s, d e)-m(d e) d s$ be the associated compensated random measure. 
Let $\mathcal{U}_{1}^{t}$ be the collection of all the $\mathbb{F}^{t}$-predictable processes in $\mathbb{L}^{2}(\Omega \times[0, T], \mathcal{F} \otimes$ $\left.\mathcal{B}[0, T], \mathbb{P} \otimes \lambda_{L} ; U_{1}\right)$, where $\lambda_{L}$ is the Lebesgue measure on $\mathbb{R}$ and $U_{1} \subset \mathbb{R}^{q}$ for some $q \in \mathbb{N}$. Define $\mathcal{U}_{2}^{t}$ to be the collection of all the maps $\nu_{2}: \Omega \times[0, T] \times E \rightarrow \mathbb{R}^{n}$ which are $\mathcal{P}^{t} \otimes \mathcal{E}$ measurable such that

$$
\left\|\nu_{2}\right\|_{\mathcal{U}_{2}^{t}}:=\left(\mathbb{E}\left[\int_{t}^{T} \int_{E}\left|\nu_{2}(s, e)\right|^{2} \hat{m}(d e) d s\right]\right)^{\frac{1}{2}}<\infty
$$

where $\mathcal{P}^{t}$ is the $\mathbb{F}^{t}$-predictable sigma-algebra on $\Omega \times[0, T] . \nu=\left(\nu_{1}, \nu_{2}\right) \in \mathcal{U}_{0}^{t}:=\mathcal{U}_{1}^{t} \times \mathcal{U}_{2}^{t}$ takes value in the set $U:=U_{1} \times \mathbb{L}^{2}\left(E, \mathcal{E}, \hat{m} ; \mathbb{R}^{n}\right)$. Let

$$
\mathbb{D}=[0, T] \times \mathbb{R}^{d}, \mathbb{D}_{i}=[0, T) \times \mathbb{R}^{d} \text { and } \mathbb{D}_{T}=\{T\} \times \mathbb{R}^{d}
$$

Given $z=(x, y) \in \mathbb{R}^{d} \times \mathbb{R}, t \in[0, T]$ and $\nu \in \mathcal{U}_{0}^{t}$, we consider the stochastic differential equations (SDEs)

$$
\begin{aligned}
d X(s) & =\mu_{X}(s, X(s), \nu(s)) d s+\sigma_{X}(s, X(s), \nu(s)) d W_{s} \\
& +\int_{E} \beta\left(s, X(s-), \nu_{1}(s), \nu_{2}(s, e), e\right) \lambda(d s, d e), \\
d Y(s) & =\mu_{Y}(s, Z(s), \nu(s)) d s+\sigma_{Y}^{\top}(s, Z(s), \nu(s)) d W_{s} \\
& +\int_{E} b^{\top}\left(s, Z(s-), \nu_{1}(s), \nu_{2}(s, e), e\right) \lambda(d s, d e),
\end{aligned}
$$

with $(X(t), Y(t))=(x, y)$. Here, $Z=(X, Y)$. In (2.2.1),

$$
\mu_{X}: \mathbb{D} \times U \rightarrow \mathbb{R}^{d}, \quad \sigma_{X}: \mathbb{D} \times U \rightarrow \mathbb{R}^{d \times d}, \quad \beta: \mathbb{D} \times U_{1} \times \mathbb{R}^{n} \times E \rightarrow \mathbb{R}^{d \times I},
$$

$\mu_{Y}: \mathbb{D} \times \mathbb{R} \times U \rightarrow \mathbb{R}, \quad \sigma_{Y}: \mathbb{D} \times \mathbb{R} \times U \rightarrow \mathbb{R}^{d}, \quad b: \mathbb{D} \times \mathbb{R} \times U_{1} \times \mathbb{R}^{n} \times E \rightarrow \mathbb{R}^{I}$.

Assumption 2.2.2. Let $z=(x, y)$ and $u=\left(u_{1}, u_{2}\right) \in U=U_{1} \times \mathbb{L}^{2}\left(E, \mathcal{E}, \hat{m} ; \mathbb{R}^{n}\right)$. We use the notation $\|u\|_{U}:=\left|u_{1}\right|+\left\|u_{2}\right\|_{\hat{m}}$ and $u(e):=\left(u_{1}, u_{2}(e)\right)$ for the rest of this chapter.

1. $\mu_{X}, \sigma_{X}, \mu_{Y}$ and $\sigma_{Y}$ are all continuous; 
2. $\mu_{X}, \sigma_{X}, \mu_{Y}, \sigma_{Y}$ are Lipschitz in $z$ and locally Lipschitz in other variables. In addition,

$$
\begin{array}{r}
\left|\mu_{X}(t, x, u)\right|+\left|\sigma_{X}(t, x, u)\right| \leq L\left(1+|x|+\|u\|_{U}\right), \\
\left|\mu_{Y}(t, x, y, u)\right|+\left|\sigma_{Y}(t, x, y, u)\right| \leq L\left(1+|y|+\|u\|_{U}\right) .
\end{array}
$$

3. $b$ and $\beta$ are Lipschitz and grow linearly in all variables except e, but uniformly in e.

Remark 2.2.3. Assumptions 2.2.1 and 2.2.2 guarantee that there exists a unique strong solution $\left(X_{t, x}^{\nu}, Y_{t, x, y}^{\nu}\right)$ to $(2.2 .1)$ for any $\nu \in \mathcal{U}_{0}^{t}$. Moreover, the processes $\left(X_{t, x}^{\nu}\right.$, $\left.Y_{t, x, y}^{\nu}\right)$ are càdlàg.

Besides the measurability and the integrability conditions for $\mathcal{U}_{0}^{t}$, we impose another condition on the admissible control set. Let $\mathcal{U}^{t}$ be the admissible control set, which consists of all $\nu \in \mathcal{U}_{0}^{t}$ such that for any compact set $C \subset \mathbb{R}^{d} \times \mathbb{R}$, there exists a constant $K_{C, \nu}>0$ such that

$$
\left|\int_{E} b^{\top}\left(\tau, x, y, \nu_{1}(\tau), \nu_{2}(\tau, e), e\right) \lambda(\{\tau\}, e)\right| \leq K_{C, \nu} \text { for }(x, y) \in C, \tau \in \mathcal{T}_{t} .
$$

We now define the value function of the stochastic target problem. Let $g: \mathbb{R}^{d} \rightarrow \mathbb{R}$ be a measurable function with polynomial growth. The value function of the target problem is defined by

$$
u(t, x):=\inf \left\{y: \exists \nu \in \mathcal{U}^{t} \text { s.t. } Y_{t, x, y}^{\nu}(T) \geq g\left(X_{t, x}^{\nu}(T)\right) \mathbb{P}-\text { a.s. }\right\} .
$$

\subsubsection{The Hamilton-Jacobi-Bellman equation}

Denote $b=\left(b_{1}, b_{2}, \cdots, b_{I}\right)^{\top}$ and $\beta=\left(\beta_{1}, \beta_{2}, \cdots, \beta_{I}\right)$. For a given $\varphi \in C(\mathbb{D})$, we define the relaxed semi-limits

$$
H^{*}(\Theta, \varphi):=\limsup _{\substack{\varepsilon \searrow 0, \Theta^{\prime} \rightarrow \Theta \\ \eta \searrow 0, \psi \stackrel{\text { u.c } \rightarrow \varphi}{\rightarrow} \varphi}} H_{\varepsilon, \eta}\left(\Theta^{\prime}, \psi\right) \text { and } H_{*}(\Theta, \varphi):=\liminf _{\substack{\varepsilon \searrow 0, \Theta^{\prime} \rightarrow \Theta \\ \eta \searrow 0, \psi \stackrel{\text { u... }}{\longrightarrow} \varphi}} H_{\varepsilon, \eta}\left(\Theta^{\prime}, \psi\right){ }^{1}
$$

\footnotetext{
${ }^{1}$ The convergence $\psi \stackrel{\text { u.c. }}{\longrightarrow} \varphi$ is understood in the sense that $\psi$ converges uniformly on compact subsets to $\varphi$.
} 
Here, for $\Theta=(t, x, y, p, A) \in \mathbb{D} \times \mathbb{R} \times \mathbb{R}^{d} \times \mathbb{M}^{d}, \varphi \in C(\mathbb{D}), \varepsilon \geq 0$ and $\eta \in[-1,1]$,

$$
\begin{gathered}
H_{\varepsilon, \eta}(\Theta, \varphi):=\sup _{u \in \mathcal{N}_{\varepsilon, \eta}(t, x, y, p, \varphi)} \mathbf{L}^{u}(\Theta), \text { where, } \\
\mathbf{L}^{u}(\Theta):=\mu_{Y}(t, x, y, u)-\mu_{X}^{\top}(t, x, u) p-\frac{1}{2} \operatorname{Tr}\left[\sigma_{X} \sigma_{X}^{\top}(t, x, u) A\right], \\
N^{u}(t, x, y, p):=\sigma_{Y}(t, x, y, u)-\sigma_{X}^{\top}(t, x, u) p, \\
\Delta^{u, e}(t, x, y, \varphi):=\min _{1 \leq i \leq I}\left\{b_{i}(t, x, y, u(e), e)-\varphi\left(t, x+\beta_{i}(t, x, u(e), e)\right)+\varphi(t, x)\right\}, \\
\mathcal{N}_{\varepsilon, \eta}(t, x, y, p, \varphi):=\left\{u \in U:\left|N^{u}(t, x, y, p)\right| \leq \varepsilon, \Delta^{u, e}(t, x, y, \varphi) \geq \eta \hat{m} \text {-a.s. } e \in E\right\} .
\end{gathered}
$$

For our later use, we also define the following:

$$
\begin{gathered}
J_{i}^{u, e}(t, x, y, \varphi):=b_{i}(t, x, y, u(e), e)-\varphi\left(t, x+\beta_{i}(t, x, u(e), e)\right)+\varphi(t, x), \\
\bar{J}^{u, e}(t, x, y, \varphi):=\left(J_{1}^{u, e}(t, x, y, \varphi), \cdots, J_{I}^{u, e}(t, x, y, \varphi)\right)^{\top} \\
\mathscr{L}^{u} \varphi(t, x):=\varphi_{t}(t, x)+\mu_{X}^{\top}(t, x, u) D \varphi(t, x)+\frac{1}{2} \operatorname{Tr}\left[\sigma_{X} \sigma_{X}^{\top}(t, x, u) D^{2} \varphi(t, x)\right] .
\end{gathered}
$$

Remark 2.2.4. For simplicity, we denote $H^{*}\left(t, x, \varphi(t, x), D \varphi(t, x), D^{2} \varphi(t, x), \varphi\right)$ by $H^{*} \varphi(t, x)$ for $\varphi \in C^{1,2}(\mathbb{D})$. For $\varphi \in C^{2}\left(\mathbb{R}^{d}\right)$, denote $H^{*}\left(T, x, \varphi(x), D \varphi(x), D^{2} \varphi(x), \varphi\right)$ by $H^{*} \varphi(x)$. We will use similar notation for $H_{*}$ and other operators in later sections.

Later, we will produce a viscosity super-solution and sub-solution, respectively, to

$$
\begin{gathered}
-\partial_{t} \varphi(t, x)+H^{*} \varphi(t, x) \geq 0 \text { in } \mathbb{D}_{i} \text { and } \\
-\partial_{t} \varphi(t, x)+H_{*} \varphi(t, x) \leq 0 \text { in } \mathbb{D}_{i} .
\end{gathered}
$$

\subsubsection{Stochastic solutions}

Before we introduce the definitions of the stochastic semi-solutions, we define the concatenation of the admissible controls.

Definition 2.2.5 (Concatenation). Let $\nu_{1}, \nu_{2} \in \mathcal{U}^{t}, \tau \in \mathcal{T}_{t}$. The concatenation of $\nu_{1}$ and $\nu_{2}$ at $\tau$ is defined as $\nu_{1} \otimes_{\tau} \nu_{2}:=\nu_{1} \mathbb{1}_{[0, \tau)}+\nu_{2} \mathbb{1}_{[\tau, T]} \in \mathcal{U}^{t}{ }^{2}$

\footnotetext{
${ }^{2}$ This can be easily checked.
} 
Definition 2.2.6 (Stochastic super-solutions). A continuous function $w: \mathbb{D} \rightarrow \mathbb{R}$ is called a stochastic super-solution if

1. $w(T, x) \geq g(x)$ and for some $C>0$ and $n \in \mathbb{N},{ }^{3}|w(t, x)| \leq C\left(1+|x|^{n}\right)$ for all $(t, x) \in \mathbb{D}$.

2. Given $(t, x, y) \in \mathbb{D} \times \mathbb{R}$, for any $\tau \in \mathcal{T}_{t}$ and $\nu \in \mathcal{U}^{t}$, there exists $\tilde{\nu} \in \mathcal{U}^{t}$ such that

$$
Y(\rho) \geq w(\rho, X(\rho)) \mathbb{P}-\text { a.s. on }\{Y(\tau) \geq w(\tau, X(\tau))\}
$$

for all $\rho \in \mathcal{T}_{\tau}$, where $X:=X_{t, x}^{\nu \otimes_{\tau} \tilde{\nu}}$ and $Y:=Y_{t, x, y}^{\nu \otimes_{\tau} \tilde{\nu}}$.

Definition 2.2.7 (Stochastic sub-solutions). A continuous function $w: \mathbb{D} \rightarrow \mathbb{R}$ is called a stochastic sub-solution if

1. $w(T, x) \leq g(x)$ and for some $C>0$ and $n \in \mathbb{N},|w(t, x)| \leq C\left(1+|x|^{n}\right)$ for all $(t, x) \in \mathbb{D}$.

2. Given $(t, x, y) \in \mathbb{D} \times \mathbb{R}$, for any $\tau \in \mathcal{T}_{t}$ and $\nu \in \mathcal{U}^{t}$, we have

$$
\mathbb{P}(Y(\rho)<w(\rho, X(\rho)) \mid B)>0
$$

for all $\rho \in \mathcal{T}_{\tau}$ and $B \subset\{Y(\tau)<w(\tau, X(\tau))\}$ satisfying $B \in \mathcal{F}_{\tau}^{t}$ and $\mathbb{P}(B)>0$. Here, we use the notation $X:=X_{t, x}^{\nu}$ and $Y:=Y_{t, x, y}^{\nu}$.

Denote the sets of stochastic super-solutions and sub-solutions by $\mathbb{U}^{+}$and $\mathbb{U}^{-}$, respectively.

Assumption 2.2.8. $\mathbb{U}^{+}$and $\mathbb{U}^{-}$are not empty.

Remark 2.2.9. Let $u^{+}:=\inf _{w \in \mathbb{U}^{+}} w$. For any stochastic super-solution $w$, choose $\tau=t$ and $\rho=T$. Then there exists $\tilde{\nu} \in \mathcal{U}^{t}$ such that $Y_{t, x, y}^{\tilde{\nu}}(T) \geq w\left(T, X_{t, x}^{\tilde{\nu}}(T)\right) \geq$ $g\left(X_{t, x}^{\tilde{\nu}}(T)\right) \mathbb{P}-$ a.s. if $y \geq w(t, x)$. Hence, $y \geq w(t, x)$ implies that $y \geq u(t, x)$ from 
(2.2.3). This means that $w \geq u$ and $u^{+} \geq u$. By the definition of $\mathbb{U}^{+}$, we know that $u^{+}(T, x) \geq g(x)$ for all $x \in \mathbb{R}^{d}$.

Remark 2.2.10. Let $u^{-}:=\sup _{w \in \mathbb{U}^{-}} w$. For any stochastic sub-solution $w$, if $y<$ $w(t, x)$, by choosing $\tau=t$ and $\rho=T$, we get that for any $\nu \in \mathcal{U}^{t}$,

$$
\mathbb{P}\left(Y_{t, x, y}^{\nu}(T)<g\left(X_{t, x}^{\nu}(T)\right)\right) \geq \mathbb{P}\left(Y_{t, x, y}^{\nu}(T)<w\left(T, X_{t, x}^{\nu}(T)\right)\right)>0
$$

Therefore, from (2.2.3), $y<w(t, x)$ implies that $y \leq u(t, x)$. This means that $w \leq u$ and $u^{-} \leq u$. By the definition of $\mathbb{U}^{-}$, it holds that $u^{-}(T, x) \leq g(x)$ for all $x \in \mathbb{R}^{d}$.

In short,

$$
u^{-}=\sup _{w \in \mathbb{U}^{-}} w \leq u \leq \inf _{w \in \mathbb{U}^{+}} w=u^{+} .
$$

We will provide sufficient conditions which guarantee Assumption 2.2.8 in the Appendix A. As in [12] and [25], the proof of the sub-solution property requires a regularity assumption on the set-valued map $\mathcal{N}_{0, \eta}(\cdot, \psi)$.

Assumption 2.2.11. For $\psi \in C(\mathbb{D}), \eta>0$, let $B$ be a subset of $\mathbb{D} \times \mathbb{R} \times \mathbb{R}^{d}$ such that $\mathcal{N}_{0, \eta}(\cdot, \psi) \neq \emptyset$ on $B$. Then for every $\varepsilon>0,\left(t_{0}, x_{0}, y_{0}, p_{0}\right) \in \operatorname{Int}(B)$ and $u_{0} \in \mathcal{N}_{0, \eta}\left(t_{0}, x_{0}, y_{0}, p_{0}, \psi\right)$, there exists an open neighborhood $B^{\prime}$ of $\left(t_{0}, x_{0}, y_{0}, p_{0}\right)$ and a locally Lipschitz continuous map $\hat{\nu}$ defined on $B^{\prime}$ such that $\left\|\hat{\nu}\left(t_{0}, x_{0}, y_{0}, p_{0}\right)-u_{0}\right\|_{U} \leq \varepsilon$ and $\hat{\nu}(t, x, y, p) \in \mathcal{N}_{0, \eta}(t, x, y, p, \psi)$.

\subsection{Viscosity property in $\mathbb{D}_{i}$}

In this section, we state and prove the theorem which characterizes $u^{+}$(resp. $\left.u^{-}\right)$as a viscosity sub-solution (resp. super-solution) of (2.2.6) (resp. (2.2.5)). The boundary conditions will be discussed in Theorem 2.4.1. Before we give the main result, we state two preparatory lemmas without proof. These two lemmas are easy to check and we refer the readers to [6] for their proofs. 
Lemma 2.3.1. $\mathbb{U}^{+}$and $\mathbb{U}^{-}$are closed under pairwise minimization and maximization, respectively. That is,

1. If $w_{1}, w_{2} \in \mathbb{U}^{+}$, then $w_{1} \wedge w_{2} \in \mathbb{U}^{+} ;$2. If $w_{1}, w_{2} \in \mathbb{U}^{-}$, then $w_{1} \vee w_{2} \in \mathbb{U}^{-}$.

Lemma 2.3.2. There exists a non-increasing sequence $\left\{w_{n}\right\}_{n=1}^{\infty} \subset \mathbb{U}^{+}$such that $w_{n} \searrow u^{+}$and a non-decreasing sequence $\left\{v_{n}\right\}_{n=1}^{\infty} \subset \mathbb{U}^{-}$such that $v_{n} \nearrow u^{-}$.

Theorem 2.3.3. Under Assumptions 2.2.1, 2.2.2, 2.2.8 and 2.2.11, $u^{+}$is a USC viscosity sub-solution of (2.2.6). On the other hand, under Assumptions 2.2.1, 2.2.2 and 2.2.8, $u^{-}$is an LSC viscosity super-solution of (2.2.5).

Proof. Step 1 ( $u^{+}$is a viscosity sub-solution). Assume, on the contrary, that for some $\left(t_{0}, x_{0}\right) \in \mathbb{D}_{i}$ and $\varphi \in C^{1,2}(\mathbb{D})$ satisfying $0=\left(u^{+}-\varphi\right)\left(t_{0}, x_{0}\right)=\max _{\mathbb{D}_{i}}\left(u^{+}-\varphi\right)$, we have

$$
4 \eta:=-\partial_{t} \varphi\left(t_{0}, x_{0}\right)+H_{*} \varphi\left(t_{0}, x_{0}\right)>0 .
$$

From Lemma 2.3.2, there exists a non-increasing sequence $\mathbb{U}^{+} \ni w_{k} \searrow u^{+}$. Fix such a sequence $\left\{w_{k}\right\}_{k=1}^{\infty}$ and an arbitrary stochastic sub-solution $w_{-}$. Let $\tilde{\varphi}(t, x)=$ $\varphi(t, x)+\iota\left|x-x_{0}\right|^{n_{0}} \cdot{ }^{4}$ We can choose $n_{0} \geq 2$ such that for any $\iota>0$,

$$
\min _{0 \leq t \leq T}\left(\tilde{\varphi}(t, x)-w_{1}(t, x)\right) \rightarrow \infty \text { as }|x| \rightarrow \infty
$$

We can do this because $\varphi(t, x)$ is bounded from below by $w_{-}$(which has polynomial growth in $x)$ and $w_{1}$ has polynomial growth in $x$. Since $\left(\mathcal{N}_{\varepsilon, \eta}\right)_{\varepsilon \geq 0}$ is non-decreasing in $\varepsilon$, we know

$$
H_{*}(\Theta, \varphi)=\liminf _{\substack{\Theta^{\prime} \rightarrow \Theta, \psi \\ \eta \searrow 0}} H_{0, \eta}\left(\Theta^{\prime}, \psi\right) .
$$

By (2.2.4) and (2.3.1), we can find $\varepsilon>0, \eta>0$ and $\iota>0$ such that for all $(t, x, y)$ satisfying $(t, x) \in B_{\varepsilon}\left(t_{0}, x_{0}\right)$ and $|y-\tilde{\varphi}(t, x)| \leq \varepsilon, \mu_{Y}(t, x, y, u)-\mathcal{L}^{u} \tilde{\varphi}(t, x) \geq$

\footnotetext{
${ }^{4}$ Since we will fix $n_{0}$ and $\iota$ later, we still use the notation $\tilde{\varphi}$ when without ambiguity despite the fact that the function depends on $n_{0}$ and $\iota$.
} 
$2 \eta$ for some $u \in \mathcal{N}_{0, \eta}(t, x, y, D \tilde{\varphi}(t, x), \tilde{\varphi})$. Fix $\iota$. Note that $\left(t_{0}, x_{0}\right)$ is still a strict maximizer of $u^{+}-\tilde{\varphi}$ over $\mathbb{D}_{i}$. For $\varepsilon$ sufficiently small, Assumption 2.2.11 implies that there exists a locally Lipschitz map $\hat{\nu}$ such that

$$
\begin{gathered}
\hat{\nu}(t, x, y, D \tilde{\varphi}(t, x)) \in \mathcal{N}_{0, \eta}(t, x, y, D \tilde{\varphi}(t, x), \tilde{\varphi}) \text { and } \\
\qquad \mu_{Y}(t, x, y, \hat{\nu}(t, x, y, D \tilde{\varphi}(t, x)))-\mathcal{L}^{\hat{\nu}(t, x, y, D \tilde{\varphi}(t, x))} \tilde{\varphi}(t, x) \geq \eta \\
\text { for all }(t, x, y) \in \mathbb{D}_{i} \times \mathbb{R} \text { s.t. }(t, x) \in B_{\varepsilon}\left(t_{0}, x_{0}\right) \text { and }|y-\tilde{\varphi}(t, x)| \leq \varepsilon .
\end{gathered}
$$

In the arguments above, choose $\varepsilon$ small enough such that $\bar{B}_{\varepsilon}\left(t_{0}, x_{0}\right) \cap \mathbb{D}_{T}=\emptyset$. Since (2.3.2) holds, there exists $R_{0}>\varepsilon$ such that $\tilde{\varphi}>w_{1}+\varepsilon \geq w_{k}+\varepsilon$ on $\mathbb{O}:=$ $\mathbb{D}-[0, T] \times \bar{B}_{R_{0}}\left(x_{0}\right)$ for all $k$. On the compact set $\mathbb{T}:=[0, T] \times \bar{B}_{R_{0}}\left(x_{0}\right)-B_{\varepsilon / 2}\left(t_{0}, x_{0}\right)$, we know that $\tilde{\varphi}>u^{+}$and the minimum of $\tilde{\varphi}-u^{+}$is attained since $u^{+}$is USC. Therefore, $\tilde{\varphi}>u^{+}+2 \alpha$ on $\mathbb{T}$ for some $\alpha>0$. By a Dini-type argument, for large enough $n$, we have $\tilde{\varphi}>w_{n}+\alpha$ on $\mathbb{T}$ and $\tilde{\varphi}>w_{n}-\varepsilon$ on $\bar{B}_{\varepsilon / 2}\left(t_{0}, x_{0}\right)$. For simplicity, fix such an $n$ and set $w=w_{n}$. In short,

$$
\tilde{\varphi}>w+\varepsilon \text { on } \mathbb{O}, \tilde{\varphi}>w+\alpha \text { on } \mathbb{T} \text { and } \tilde{\varphi}>w-\varepsilon \text { on } \bar{B}_{\varepsilon / 2}\left(t_{0}, x_{0}\right)
$$

For $\kappa \in(0, \varepsilon \wedge \alpha)$, define

$$
w^{\kappa}:=\left\{\begin{array}{l}
(\tilde{\varphi}-\kappa) \wedge w \text { on } \bar{B}_{\varepsilon}\left(t_{0}, x_{0}\right), \\
w \text { outside } \bar{B}_{\varepsilon}\left(t_{0}, x_{0}\right) .
\end{array}\right.
$$

Observing that $w^{\kappa}\left(t_{0}, x_{0}\right)=\tilde{\varphi}\left(t_{0}, x_{0}\right)-\kappa<u^{+}\left(t_{0}, x_{0}\right)$, we could obtain a contradiction if we could show that $w^{\kappa} \in \mathbb{U}^{+}$. Obviously, $w^{\kappa}$ is continuous, has polynomial growth in $x$ and $w^{\kappa}(T, x) \geq g(x)$ for all $x \in \mathbb{R}^{d}$.

Fix $(t, x, y) \in \mathbb{D}_{i} \times \mathbb{R}, \nu \in \mathcal{U}^{t}$ and $\tau \in \mathcal{T}_{t}{ }^{5}$. Now our goal is to construct an admissible control $\widetilde{\nu}$ such that $w^{\kappa}$ and the processes $(X, Y)$ controlled by $\nu \otimes_{\tau} \widetilde{\nu}$ satisfy the property in the definition of stochastic super-solutions.

\footnotetext{
${ }^{5}$ Here we choose $(t, x) \in \mathbb{D}_{i}$ since the case $(t, x) \in \mathbb{D}_{T}$ is trivial.
} 
Let $A=\left\{w^{\kappa}\left(\tau, X_{t, x}^{\nu}(\tau)\right)=w\left(\tau, X_{t, x}^{\nu}(\tau)\right)\right\}$. On $A$, let $\widetilde{\nu}$ be $\widetilde{\nu}_{1}$, which is "optimal" for $w$ starting at $\tau$. We get the existence of $\widetilde{\nu}_{1}$ since $w \in \mathbb{U}^{+}$. On $A^{c}$, by an argument similar to that in [6] (see Step 1.1 of Theorem 3.1's proof), we can construct an admissible control $\nu_{0} \in \mathcal{U}^{t}$ such that

$$
\begin{aligned}
\nu_{0}(s):= & \hat{\nu}\left(s, X_{t, x}^{\nu \otimes_{\tau} \nu_{0}}(s), Y_{t, x, y}^{\nu \otimes_{\tau} \nu_{0}}(s), D \tilde{\varphi}\left(s, X_{t, x}^{\nu \otimes_{\tau} \nu_{0}}(s)\right) \text { for } \tau \leq s<\theta:=\theta_{1} \wedge \theta_{2},\right. \\
& \text { where } \theta_{1}:=\inf \left\{s \in[\tau, T]:\left(s, X_{t, x}^{\nu \otimes_{\tau} \nu_{0}}(s)\right) \notin B_{\varepsilon / 2}\left(t_{0}, x_{0}\right)\right\} \wedge T, \\
& \theta_{2}:=\inf \left\{s \in[\tau, T]:\left|Y_{t, x, y}^{\nu \otimes_{\tau} \nu_{0}}(s)-\tilde{\varphi}\left(s, X_{t, x}^{\nu \otimes_{\tau} \nu_{0}}(s)\right)\right| \geq \varepsilon\right\} \wedge T .
\end{aligned}
$$

In the construction of $\nu_{0}$, we take advantage of Assumption 2.2.2 and the Lipschitz continuity of $\hat{\nu}$ which guarantee the existence of $X_{t, x}^{\nu \otimes_{\tau} \nu_{0}}$ and $Y_{t, x, y}^{\nu \otimes_{\tau} \nu_{0}}$. Since $X_{t, x}^{\nu \otimes_{\tau} \nu_{0}}$ and $Y_{t, x, y}^{\nu \otimes_{\tau} \nu_{0}}$ are càdlàg, it is easy to check that $\theta \in \mathcal{T}_{\tau}$. We also see that

$$
\begin{gathered}
\left(\theta_{1}, X_{t, x}^{\nu \otimes_{\tau} \nu_{0}}\left(\theta_{1}\right)\right) \notin B_{\varepsilon / 2}\left(t_{0}, x_{0}\right), \quad\left|Y_{t, x, y}^{\nu \otimes_{\tau} \nu_{0}}\left(\theta_{2}\right)-\tilde{\varphi}\left(\theta_{2}, X_{t, x}^{\nu \otimes_{\tau} \nu_{0}}\left(\theta_{2}\right)\right)\right| \geq \varepsilon \\
\left(\theta_{1}, X_{t, x}^{\nu \otimes_{\tau} \nu_{0}}\left(\theta_{1}-\right)\right) \in \bar{B}_{\varepsilon / 2}\left(t_{0}, x_{0}\right), \quad\left|Y_{t, x, y}^{\nu \otimes_{\tau} \nu_{0}}\left(\theta_{2}-\right)-\tilde{\varphi}\left(\theta_{2}, X_{t, x}^{\nu \otimes_{\tau} \nu_{0}}\left(\theta_{2}-\right)\right)\right| \leq \varepsilon
\end{gathered}
$$

Let $\widetilde{\nu}^{\theta}$ be the "optimal" control for $w$ starting at $\theta$. We define $\tilde{\nu}$ on $A^{c}$ by $\nu_{0} \otimes_{\theta} \widetilde{\nu}^{\theta}$. In short,

$$
\widetilde{\nu}:=\left(\mathbb{1}_{A} \widetilde{\nu}_{1}+\mathbb{1}_{A^{c}}\left(\nu_{0} \mathbb{1}_{[t, \theta[}+\mathbb{1}_{[\theta, T]} \widetilde{\nu}^{\theta}\right)\right) \mathbb{1}_{[\tau, T]}
$$

It is not difficult to check that $\widetilde{\nu} \in \mathcal{U}^{t}$. To prove that the above construction works, we next show that

$$
Y(\rho) \geq w^{\kappa}(\rho, X(\rho)) \text { on } B:=\left\{Y(\tau) \geq w^{\kappa}(\tau, X(\tau))\right\}
$$

where $X:=X_{t, x}^{\nu \otimes_{\tau} \tilde{\nu}}$ and $Y:=Y_{t, x, y}^{\nu \otimes_{\tau} \tilde{\nu}}$. Corresponding to the construction of $\widetilde{\nu}$ on $A$ and $A^{c}$, we consider the following two cases:

(i) On the set $A \cap B$. We have $Y(\tau) \geq w(\tau, X(\tau))$. From the definition of $\nu$ on $A$ and the fact that $w \in \mathbb{U}^{+}$, we know

$$
Y(\rho)=Y_{t, x, y}^{\nu \otimes_{\tau} \widetilde{\nu}_{1}}(\rho) \geq w\left(\rho, X_{t, x}^{\nu \otimes_{\tau} \widetilde{\nu}_{1}}(\rho)\right) \geq w^{\kappa}(\rho, X(\rho)) \text { on } A \cap B
$$


(ii) On the set $A^{c} \cap B$. Letting $\Gamma(s):=Y(s)-\tilde{\varphi}(s, X(s))$, we use Itô's formula and the definition of $\nu_{0}$ to obtain

$$
\begin{aligned}
\Gamma(\cdot \wedge \theta) & =\Gamma(\tau)+\int_{\tau}^{\cdot \wedge \theta} \int_{E} \bar{J}^{\nu_{0}(s), e}(s, Z(s-), \tilde{\varphi})^{\top} \lambda(d s, d e) \\
& +\int_{\tau}^{\cdot \wedge \theta}\left(\mu_{Y}\left(s, Z(s), \nu_{0}(s)\right)-\mathscr{L}^{\nu_{0}(s)} \tilde{\varphi}(s, X(s))\right) d s \text { on } A^{c} \cap B
\end{aligned}
$$

Therefore, by $(2.3 .3),(2.3 .4),(2.3 .7)$ and the definition of $\theta$, we know that $\Gamma(\cdot \wedge \theta)$ is non-decreasing on $[\tau, T]$. This implies that

$$
Y(\theta)-\tilde{\varphi}(\theta, X(\theta))+\kappa \geq Y(\tau)-\tilde{\varphi}(\tau, X(\tau))+\kappa \geq 0 \text { on } A^{c} \cap B
$$

Since $\left(\theta_{1}, X\left(\theta_{1}\right)\right) \notin B_{\varepsilon / 2}\left(t_{0}, x_{0}\right)$, we know from $(2.3 .5)$

$$
0 \leq Y\left(\theta_{1}\right)-\tilde{\varphi}\left(\theta_{1}, X\left(\theta_{1}\right)\right)+\kappa \leq Y\left(\theta_{1}\right)-w\left(\theta_{1}, X\left(\theta_{1}\right)\right) \text { on }\left\{\theta_{1} \leq \theta_{2}\right\} \cap A^{c} \cap B
$$

On the other hand, due to (2.3.6) and (2.3.8), it holds that

$$
Y\left(\theta_{2}\right)-\tilde{\varphi}\left(\theta_{2}, X\left(\theta_{2}\right)\right) \geq \varepsilon \text { on }\left\{\theta_{1}>\theta_{2}\right\} \cap A^{c} \cap B
$$

Therefore, since $\tilde{\varphi}>w-\varepsilon$ on $\bar{B}_{\varepsilon / 2}\left(t_{0}, x_{0}\right)$ and (2.3.7) holds,

$Y\left(\theta_{2}\right)-w\left(\theta_{2}, X\left(\theta_{2}\right)\right) \geq \varepsilon+\tilde{\varphi}\left(\theta_{2}, X\left(\theta_{2}\right)\right)-w\left(\theta_{2}, X\left(\theta_{2}\right)\right)>0$ on $\left\{\theta_{1}>\theta_{2}\right\} \cap A^{c} \cap B$.

Combining the equation above and (2.3.9), we obtain $Y(\theta)-w(\theta, X(\theta)) \geq 0$ on $A^{c} \cap B$. Therefore, from the definition of $\widetilde{\nu}^{\theta}$,

$$
Y(\rho \vee \theta)-w^{\kappa}(\rho \vee \theta, X(\rho \vee \theta)) \geq Y(\rho \vee \theta)-w(\rho \vee \theta, X(\rho \vee \theta)) \geq 0 \text { on } A^{c} \cap B
$$

Also, the monotonicity of $\Gamma(\cdot \wedge \theta)$ implies that

$$
Y(\rho \wedge \theta)-\tilde{\varphi}(\rho \wedge \theta, X(\rho \wedge \theta))+\kappa \geq 0 \text { on } A^{c} \cap B
$$

This means that

$$
\mathbb{1}_{\{\rho<\theta\}}\left(Y(\rho)-w^{\kappa}(\rho, X(\rho))\right) \geq 0 \text { on } A^{c} \cap B
$$


From (2.3.10) and (2.3.11), we get $Y(\rho)-w^{\kappa}(\rho, X(\rho)) \geq 0$ on $A^{c} \cap B$.

Step $2\left(u^{-}\right.$is a viscosity super-solution). Let $\left(t_{0}, x_{0}\right) \in \mathbb{D}_{i}$ satisfy $0=\left(u^{-}-\right.$ $\varphi)\left(t_{0}, x_{0}\right)=\min _{\mathbb{D}_{i}}\left(u^{-}-\varphi\right)$ for some $\varphi \in C^{1,2}(\mathbb{D})$. For the sake of contradiction, assume that

$$
-2 \eta:=-\partial_{t} \varphi\left(t_{0}, x_{0}\right)+H^{*} \varphi\left(t_{0}, x_{0}\right)<0
$$

Let $\left\{w_{k}\right\}_{k=1}^{\infty}$ be a sequence in $\mathbb{U}^{-}$such that $w_{k} \nearrow u^{-}$and $\tilde{\varphi}(t, x):=\varphi(t, x)-\iota \mid x-$ $\left.x_{0}\right|^{n_{0}}$, where we choose $n_{0} \geq 2$ such that for all $\iota>0$,

$$
\max _{0 \leq t \leq T}\left(\tilde{\varphi}(t, x)-w_{1}(t, x)\right) \rightarrow-\infty \text { and } \max _{0 \leq t \leq T} \tilde{\varphi}(t, x) \rightarrow-\infty \text { as }|x| \rightarrow \infty .^{6}
$$

By (2.3.12), the upper semi-continuity of $H^{*}$ and the fact that $\tilde{\varphi} \stackrel{\text { u.c. }}{\longrightarrow} \varphi$ as $\iota \rightarrow 0$, we can find $\varepsilon>0, \eta>0$ and $\iota>0$ such that

$$
\begin{aligned}
& \mu_{Y}(t, x, y, u)-\mathscr{L}^{u} \tilde{\varphi}(t, x) \leq-\eta \text { for all } u \in \mathcal{N}_{\varepsilon,-\eta}(t, x, y, D \tilde{\varphi}(t, x), \tilde{\varphi}) \\
& \text { and }(t, x, y) \in \mathbb{D}_{i} \times \mathbb{R} \text { s.t. }(t, x) \in B_{\varepsilon}\left(t_{0}, x_{0}\right) \text { and }|y-\tilde{\varphi}(t, x)| \leq \varepsilon .
\end{aligned}
$$

Fix $\iota$. Note that $\left(t_{0}, x_{0}\right)$ is still a strict minimizer of $u^{-}-\tilde{\varphi}$. Since $(2.3 .13)$ holds, there exists $R_{0}>\varepsilon$ such that

$$
\tilde{\varphi}<w_{1}-\varepsilon \leq w_{k}-\varepsilon \text { on } \mathbb{O}:=\mathbb{D}-[0, T] \times \bar{B}_{R_{0}}\left(x_{0}\right)
$$

On the compact set $\mathbb{T}:=[0, T] \times \bar{B}_{R_{0}}\left(x_{0}\right)-B_{\varepsilon / 2}\left(t_{0}, x_{0}\right)$, we know that $\tilde{\varphi}<u^{-}$and the maximum of $\tilde{\varphi}-u^{-}$is attained since $u^{-}$is LSC. Therefore, $\tilde{\varphi}<u^{-}-2 \alpha$ on $\mathbb{T}$ for some $\alpha>0$. By a Dini-type argument, for large enough $n$, we have $\tilde{\varphi}<w_{n}-\alpha$ on $\mathbb{T}$ and $\tilde{\varphi}<w_{n}+\varepsilon$ on $\bar{B}_{\varepsilon / 2}\left(t_{0}, x_{0}\right)$. For simplicity, fix such an $n$ and set $w=w_{n}$. In short,

$$
\tilde{\varphi}<w-\varepsilon \text { on } \mathbb{O}, \tilde{\varphi}<w-\alpha \text { on } \mathbb{T} \text { and } \tilde{\varphi}<w+\varepsilon \text { on } \bar{B}_{\varepsilon / 2}\left(t_{0}, x_{0}\right) .
$$

\footnotetext{
${ }^{6}$ The existence of $n_{0}$ follows as in Step1.
} 
For $\kappa \in(0, \alpha \wedge \varepsilon)$, define

$$
w^{\kappa}:=\left\{\begin{array}{l}
(\tilde{\varphi}+\kappa) \vee w \text { on } \bar{B}_{\varepsilon}\left(t_{0}, x_{0}\right), \\
w \text { outside } \bar{B}_{\varepsilon}\left(t_{0}, x_{0}\right) .
\end{array}\right.
$$

Noticing that $w^{\kappa}\left(t_{0}, x_{0}\right) \geq \tilde{\varphi}\left(t_{0}, x_{0}\right)+\kappa>u^{-}\left(t_{0}, x_{0}\right)$, we will obtain a contradiction if we show that $w^{\kappa} \in \mathbb{U}^{-}$. Obviously, $w^{\kappa}$ is continuous, has polynomial growth in $x$ and $w^{\kappa}(T, x) \leq g(x)$ for all $x \in \mathbb{R}^{d}$. Fix $(t, x, y) \in \mathbb{D}_{i} \times \mathbb{R}, \nu \in \mathcal{U}^{t}$ and $\tau \in \mathcal{T}_{t}$. Our goal is to show that

$$
\mathbb{P}\left(Y(\rho)<w^{\kappa}(\rho, X(\rho)) \mid B\right)>0
$$

for all $\rho \in \mathcal{T}_{\tau}$ and $B \subset\left\{Y(\tau)<w^{\kappa}(\tau, X(\tau))\right\}$ satisfying $B \in \mathcal{F}_{\tau}^{t}$ and $\mathbb{P}(B)>0$, where $X:=X_{t, x}^{\nu}$ and $Y:=Y_{t, x, y}^{\nu}$. Let $A=\left\{w^{\kappa}(\tau, X(\tau))=w(\tau, X(\tau))\right\}$ and set

$$
\begin{gathered}
E=\left\{Y(\tau)<w^{\kappa}(\tau, X(\tau))\right\}, \quad E_{0}=E \cap A, \quad E_{1}=E \cap A^{c}, \\
G=\left\{Y(\rho)<w^{\kappa}(\rho, X(\rho)\}, \quad G_{0}=\{Y(\rho)<w(\rho, X(\rho)\} .\right.
\end{gathered}
$$

Then $E=E_{0} \cup E_{1}, E_{0} \cap E_{1}=\emptyset$ and $G_{0} \subset G$. To prove that $w^{\kappa} \in \mathbb{U}^{-}$, it suffices to show that $\mathbb{P}(G \cap B)>0$. As in [33], we will show

$$
\mathbb{P}\left(B \cap E_{0}\right)>0 \Longrightarrow \mathbb{P}\left(G \cap B \cap E_{0}\right)>0, \mathbb{P}\left(B \cap E_{1}\right)>0 \Longrightarrow \mathbb{P}\left(G \cap B \cap E_{1}\right)>0 .
$$

This, together with the facts $\mathbb{P}(B)=\mathbb{P}\left(B \cap E_{0}\right)+\mathbb{P}\left(B \cap E_{1}\right)>0$ and $\mathbb{P}(G \cap B)=$ $\mathbb{P}\left(G \cap B \cap E_{0}\right)+\mathbb{P}\left(G \cap B \cap E_{1}\right)$, implies that $\mathbb{P}(G \cap B)>0$.

(i)Assume that $\mathbb{P}\left(B \cap E_{0}\right)>0$. Since $B \cap E_{0} \subset\{Y(\tau)<w(\tau, X(\tau))\}$ and $B \cap E_{0} \in \mathcal{F}_{\tau}^{t}, \mathbb{P}\left(G_{0} \mid B \cap E_{0}\right)>0$ from the definition of $\mathbb{U}^{-}$. This further implies that $\mathbb{P}\left(G \cap B \cap E_{0}\right) \geq \mathbb{P}\left(G_{0} \cap B \cap E_{0}\right)>0$.

(ii) Assume that $\mathbb{P}\left(B \cap E_{1}\right)>0$. Let $\theta=\theta_{1} \wedge \theta_{2}$, where

$$
\begin{aligned}
& \theta_{1}:=\inf \left\{s \in[\tau, T]:(s, X(s)) \notin B_{\varepsilon / 2}\left(t_{0}, x_{0}\right)\right\} \wedge T, \\
& \theta_{2}:=\inf \{s \in[\tau, T]:|Y(s)-\tilde{\varphi}(s, X(s))| \geq \varepsilon\} \wedge T .
\end{aligned}
$$


Since $X$ and $Y$ are càdlàg processes, we know that $\theta \in \mathcal{T}_{\tau}$. The following also hold:

$$
\begin{gathered}
\left(\theta_{1}, X\left(\theta_{1}\right)\right) \notin B_{\varepsilon / 2}\left(t_{0}, x_{0}\right), \quad\left|Y\left(\theta_{2}\right)-\tilde{\varphi}\left(\theta_{2}, X\left(\theta_{2}\right)\right)\right| \geq \varepsilon, \\
\left(\theta_{1}, X\left(\theta_{1}-\right)\right) \in \bar{B}_{\varepsilon / 2}\left(t_{0}, x_{0}\right), \quad\left|Y\left(\theta_{2}-\right)-\tilde{\varphi}\left(\theta_{2}, X\left(\theta_{2}-\right)\right)\right| \leq \varepsilon .
\end{gathered}
$$

Let

$$
\begin{gathered}
a(s)=\mu_{Y}(s, X(s), Y(s), \nu(s))-\mathscr{L}^{\nu(s)} \tilde{\varphi}(s, X(s)), \\
c_{i}^{e}(s)=J_{i}^{u, e}(s, X(s-), Y(s-), \tilde{\varphi}), d_{i}(s)=\int_{E} c_{i}^{e}(s) m_{i}(d e), d(s)=\sum_{i=1}^{I} d_{i}(s), \\
\pi(s)=N^{\nu(s)}(s, X(s), Y(s), D \tilde{\varphi}(s, X(s))), A_{0}=\{s \in[\tau, \theta]:|\pi(s)| \leq \varepsilon\}, \\
A_{1}=\left\{s \in[\tau, \theta]: c_{i}^{e}(s) \geq-\eta \text { for } \hat{m}-\text { a.s. } e \in E \text { for all } i=1, \cdots, I\right\}, A_{2}=\left(A_{1}\right)^{c}, \\
A_{3, i}=\left\{(s, e) \in[\tau, \theta] \times E: c_{i}^{e}(s) \leq-\eta / 2\right\} .
\end{gathered}
$$

We then set

$$
L(\cdot):=\mathcal{E}\left(\int_{t}^{\cdot \wedge \theta} \int_{E} \sum \delta_{i}^{e}(s) \tilde{\lambda}_{i}(d s, d e)+\int_{t}^{\cdot \wedge \theta} \alpha^{\top}(s) d W_{s}\right),
$$

where $\mathcal{E}(\cdot)$ denotes the Doléans-Dade exponential and

$$
\begin{gathered}
x^{+}:=\max \{0, x\}, \quad x^{-}:=\max \{0,-x\}, \quad \alpha(s):=-\frac{a(s)+d(s)}{|\pi(s)|^{2}} \pi(s) \mathbb{1}_{A_{0}^{c}}(s), \\
M_{i}(s):=\int_{E} \mathbb{1}_{A_{3, i}}(s, e) m_{i}(d e), \quad K_{i}(s, e):= \begin{cases}\frac{\mathbb{1}_{A_{3, i}}(s, e)}{M_{i}(s)} & \text { if } M_{i}(s)=0 \\
0 & \text { otherwise }\end{cases} \\
\delta_{i}^{e}(s):=\left(\frac{\eta}{2(1+|d(s)|)}-1+\mathbb{1}_{A_{2}}(s) \cdot \frac{2 a(s)^{+}+\eta}{\eta} \cdot K_{i}(s, e)\right) \mathbb{1}_{A_{0}}(s) .
\end{gathered}
$$

If $s \in A_{2}$, then it follows from Assumption 2.2.1 and definitions of $A_{2}$ and $A_{3, i}$ that

$$
M_{i_{0}}(s)>0 \text { for some } i_{0} \in\{1,2, \cdots, I\} \text {. }
$$

Obviously, $L$ is a nonnegative local martingale on $[t, T]$. Therefore, it is a super- 
martingale. Let $\Gamma(s):=Y(s)-\tilde{\varphi}(s, X(s))-\kappa$. Applying Itô's formula, we get

$$
\begin{aligned}
\Gamma(\cdot \wedge \theta) L(\cdot \wedge \theta) & =\Gamma(\tau) L(\tau)+\int_{\tau}^{\cdot \wedge \theta} L(s)(\pi(s)+\Gamma(s) \alpha(s))^{\top} d W_{s} \\
& +\int_{\tau}^{\cdot \wedge \theta} \int_{E} \sum L(s)\left\{c_{i}^{e}(s)+\Gamma(s) \delta_{i}^{e}(s)+c_{i}^{e}(s) \delta_{i}^{e}(s)\right\} \tilde{\lambda}(d s, d e) \\
& +\int_{\tau}^{\cdot \wedge \theta} L(s)\left\{(a(s)+d(s)) \mathbb{1}_{A_{0}}(s)+\int_{E} \sum c_{i}^{e}(s) \delta_{i}^{e}(s) m_{i}(d e)\right\} d s
\end{aligned}
$$

By the definition of $\delta_{i}^{e}$ and the fact that $\mathbb{1}_{A_{1}}+\mathbb{1}_{A_{2}}=1$ on $[\tau, \theta]$, the last integral in the equation above is

$$
\begin{aligned}
& \int_{\tau}^{\cdot \wedge \theta} L(s)\left\{\left(a(s)+\frac{\eta d(s)}{2(|d(s)|+1)}\right) \mathbb{1}_{A_{0} \cap A_{1}}(s)+\mathbb{1}_{A_{0} \cap A_{2}}(s)\right. \\
& \left.\times\left(a(s)+\frac{\eta d(s)}{2(|d(s)|+1)}+\frac{2 a(s)^{+}+\eta}{\eta} \int_{E} \sum c_{i}^{e}(s) K_{i}(s, e) m_{i}(d e)\right)\right\} d s .
\end{aligned}
$$

By (2.3.14), $a(s) \leq-\eta$ on $A_{0} \cap A_{1}$. Then,

$$
\left(a(s)+\frac{\eta d(s)}{2(|d(s)|+1)}\right) \mathbb{1}_{A_{0} \cap A_{1}}(s) \leq\left(-\eta+\frac{\eta}{2}\right) \mathbb{1}_{A_{0} \cap A_{1}}(s) \leq 0 .
$$

By the definition of $A_{3, i}$ and (2.3.18), it holds that

$$
\begin{aligned}
& \mathbb{1}_{A_{0} \cap A_{2}}(s)\left(a(s)+\frac{\eta d(s)}{2(|d(s)|+1)}+\frac{2 a(s)^{+}+\eta}{\eta} \int_{E} \sum c_{i}^{e}(s) K_{i}(s, e) m_{i}(d e)\right) \\
\leq & \mathbb{1}_{A_{0} \cap A_{2}}(s)\left(a(s)+\frac{\eta}{2}-\frac{2 a(s)^{+}+\eta}{\eta} \cdot \frac{\eta}{2}\right)=-\mathbb{1}_{A_{0} \cap A_{2}}(s) a(s)^{-} .
\end{aligned}
$$

Therefore, (2.3.19) and (2.3.20) imply that $\Gamma L$ is a local super-martingale on $[\tau, \theta]$. Note that

$$
\Gamma(\theta)-\Gamma(\theta-)=\int_{E} \bar{J}^{\nu(\theta), e}(\theta, X(\theta-), Y(\theta-), \tilde{\varphi})^{\top} \lambda(\{\theta\}, d e) .
$$

Since $\tilde{\varphi} \in C(\mathbb{D})$ and $(2.3 .13)$ holds, $\tilde{\varphi}$ is locally bounded and globally bounded from above. This, together with (2.3.17) and the admissibility condition (2.2.2), implies that $\Gamma(\theta)-\Gamma(\theta-) \geq-K$ almost surely for some $K>0$ ( $K$ may depend on $\left(t_{0}, x_{0}\right), \varepsilon$, $\nu$ and $\tilde{\varphi})$. Since $\Gamma(s)=Y(s)-\tilde{\varphi}(s, X(s))-\kappa \geq-(\varepsilon+\kappa)$ on $[\tau, \theta), \Gamma L$ is bounded 
from below by a sub-martingale $-(\varepsilon+\kappa+K) L$ on $[\tau, \theta]$. This further implies that $\Gamma L$ is a super-martingale by Fatou's Lemma. Since $\Gamma(\tau) L(\tau)<0$ on $B \cap E_{1}$, the super-martingale property implies that there exists $F \subset B \cap E_{1}$ such that $F \in \mathcal{F}_{\tau}^{t}$ and $\Gamma(\theta \wedge \rho) L(\theta \wedge \rho)<0$ on $F$. The non-negativity of $L$ then yields $\Gamma(\theta \wedge \rho)<0$. Therefore,

$$
\begin{gathered}
Y\left(\theta_{1}\right)<\tilde{\varphi}\left(\theta_{1}, X\left(\theta_{1}\right)\right)+\kappa \text { on } F \cap\left\{\theta_{1} \leq \theta_{2}, \theta<\rho\right\}, \\
Y\left(\theta_{2}\right)<\tilde{\varphi}\left(\theta_{2}, X\left(\theta_{2}\right)\right)+\kappa \text { on } F \cap\left\{\theta_{1}>\theta_{2}, \theta<\rho\right\}, \\
Y(\rho)-(\tilde{\varphi}(\rho, X(\rho))+\kappa)<0 \text { on } F \cap\{\theta \geq \rho\} .
\end{gathered}
$$

Since $\left(\theta_{1}, X\left(\theta_{1}\right)\right) \notin B_{\varepsilon / 2}\left(t_{0}, x_{0}\right)$, it follows from the first two inequalities in $(2.3 .15)$ that

$$
Y\left(\theta_{1}\right)<\tilde{\varphi}\left(\theta_{1}, X\left(\theta_{1}\right)\right)+\kappa<w\left(\theta_{1}, X\left(\theta_{1}\right)\right) \text { on } F \cap\left\{\theta_{1} \leq \theta_{2}, \theta<\rho\right\}
$$

On the other hand, since $Y\left(\theta_{2}\right)<\tilde{\varphi}\left(\theta_{2}, X\left(\theta_{2}\right)\right)+\kappa$ on $F \cap\left\{\theta_{1}>\theta_{2}, \theta<\rho\right\}$ and (2.3.16) holds,

$$
Y\left(\theta_{2}\right)-\tilde{\varphi}\left(\theta_{2}, X\left(\theta_{2}\right)\right) \leq-\varepsilon \text { on } F \cap\left\{\theta_{1}>\theta_{2}, \theta<\rho\right\}
$$

Observing that $\left(\theta_{2}, X\left(\theta_{2}\right)\right) \in B_{\varepsilon / 2}\left(t_{0}, x_{0}\right)$ on $\left\{\theta_{1}>\theta_{2}\right\}$, we get from the last inequality of $(2.3 .15)$ that

$$
Y\left(\theta_{2}\right) \leq \tilde{\varphi}\left(\theta_{2}, X\left(\theta_{2}\right)\right)-\varepsilon<w\left(\theta_{2}, X\left(\theta_{2}\right)\right) \text { on } F \cap\left\{\theta_{1}>\theta_{2}, \theta<\rho\right\}
$$

From (2.3.22) and (2.3.23), we get that $Y(\theta)<w(\theta, X(\theta))$ on $F \cap\{\theta<\rho\}$. Therefore, from the definition of $\mathbb{U}^{-}$,

$$
\mathbb{P}\left(G_{0} \mid F \cap\{\theta<\rho\}\right)>0 \text { if } \mathbb{P}(F \cap\{\theta<\rho\})>0 .
$$

From (2.3.21), it holds that

$$
\mathbb{P}(G \mid F \cap\{\theta \geq \rho\})>0 \text { if } \mathbb{P}(F \cap\{\theta \geq \rho\})>0
$$


Since $G_{0} \subset G,(2.3 .24)$ and (2.3.25) imply that $\mathbb{P}(G \cap F)>0$. Therefore,

$$
\mathbb{P}\left(G \cap B \cap E_{1}\right)>0 .
$$

\subsection{Boundary conditions}

In this section, we discuss the boundary conditions at $T$. From the definition of the value function $u$, it holds that $u(T, x)=g(x)$ for all $x \in \mathbb{R}^{d}$. However, $u^{+}$and $u^{-}$ may not satisfy this boundary condition. Define

$\mathbf{N}(t, x, y, p, \psi):=\left\{(r, s): \exists u \in U\right.$ s.t. $r=N^{u}(t, x, y, p), s \leq \Delta^{u, e}(t, x, y, \psi) \hat{m}$-a.s. $\}$

and $\delta:=\operatorname{dist}\left(0, \mathbf{N}^{c}\right)-\operatorname{dist}(0, \mathbf{N})$, where dist denotes the Euclidean distance. It holds that

$$
0 \in \operatorname{int}(N(t, x, y, p, \psi)) \text { iff } \delta(t, x, y, p, \psi)>0
$$

The upper (resp. lower) semi-continuous envelope of $\delta$ is denoted by $\delta^{*}$ (resp. $\delta_{*}$ ). Let

$$
u^{+}(T-, x)=\limsup _{\left(t<T, x^{\prime}\right) \rightarrow(T, x)} u^{-}\left(t, x^{\prime}\right), \quad u^{-}(T-, x)=\liminf _{\left(t<T, x^{\prime}\right) \rightarrow(T, x)} u^{-}\left(t, x^{\prime}\right) .
$$

The following theorem is an adaptation of the results in $[32,33,12,13]$.

Theorem 2.4.1. Under Assumptions 2.2.1, 2.2.2, 2.2.8 and 2.2.11, if $g$ is $U S C$, then $u^{+}(T-, \cdot)$ is a USC viscosity sub-solution of

$$
\min \left\{\varphi(x)-g(x), \delta_{*} \varphi(x)\right\} \leq 0 \text { on } \mathbb{R}^{d}
$$

On the other hand, under Assumptions 2.2.1, 2.2.2 and 2.2.8, if $g$ is $L S C$, $u^{-}(T-, \cdot)$ is an LSC viscosity super-solution of

$$
\min \left\{(\varphi(x)-g(x)) \mathbb{1}_{\left\{H^{*} \varphi(x)<\infty\right\}}, \delta^{*} \varphi(x)\right\} \geq 0 \text { on } \mathbb{R}^{d}
$$


Proof Step 1 (The sub-solution property on $\mathbb{D}_{T}$ ). For the sake of contradiction, we assume that for some $x_{0} \in \mathbb{R}^{d}$ and $\varphi \in C^{2}\left(\mathbb{R}^{d}\right)$ satisfying $0=u^{+}\left(T-, x_{0}\right)-\varphi\left(x_{0}\right)=$ $\max _{x \in \mathbb{R}^{d}}\left(u^{+}(T-, x)-\varphi(x)\right)$, it holds that

$$
\varphi\left(x_{0}\right)-g\left(x_{0}\right)>2 \eta \text { and } \delta_{*} \varphi\left(x_{0}\right)>2 \eta \text { for some } \eta>0 \text {. }
$$

Let $\left\{w_{k}\right\}_{k=1}^{\infty}$ be a sequence in $\mathbb{U}^{+}$such that $w_{k} \searrow u^{+}$. Set $\tilde{\varphi}(t, x)=\varphi(x)+\iota \mid x-$ $\left.x_{0}\right|^{n_{0}}+\iota \sqrt{T-t}$ for $\iota>0$, where $\iota$ will be fixed later and $n_{0}$ satisfies

$$
\min _{0 \leq t \leq T}\left(\tilde{\varphi}(t, x)-w_{1}(t, x)\right) \rightarrow \infty \text { as }|x| \rightarrow \infty \text { for any } \iota>0
$$

By the lower semi-continuity of $\delta_{*}$ and the upper semi-continuity of $g$, we can find $\iota>0$ and $\varepsilon>0$ such that for $(t, x) \in[T-\varepsilon, T] \times \bar{B}_{\varepsilon}\left(x_{0}\right)$ and $y \in \mathbb{R}$ satisfying $|y-\tilde{\varphi}(t, x)| \leq \varepsilon$

$$
\begin{gathered}
\tilde{\varphi}(t, x)-g(x)>\eta \text { and } \\
\delta_{*}(t, x, y, D \tilde{\varphi}(t, x), \tilde{\varphi}) \geq \eta .
\end{gathered}
$$

By Assumption 2.2.11, the fact that $\delta \geq \delta_{*},(2.4 .1)$ and (2.4.3), we can find a locally Lipschitz map $\hat{\nu}$ such that

$$
\begin{gathered}
\hat{\nu}(t, x, y, D \tilde{\varphi}(t, x)) \in \mathcal{N}_{0, \eta}(t, x, y, \tilde{\varphi}(t, x), \tilde{\varphi}) \text { for all } \\
\left.(t, x, y) \in \mathbb{D} \times \mathbb{R} \text { s.t. }(t, x) \in[T-\varepsilon, T] \times \bar{B}_{\varepsilon}\left(x_{0}\right)\right) \text { and }|y-\tilde{\varphi}(t, x)| \leq \varepsilon
\end{gathered}
$$

In (2.4.4), we may need to choose smaller values of $\varepsilon, \iota$ and $\eta$. Fix $\iota$. Since $\partial_{t} \tilde{\varphi}(t, x) \rightarrow$ $-\infty$ as $t \rightarrow T$, by the continuity of $\mu_{Y}, \mu_{X}, \sigma_{X}$ and $\nu$,

$$
\begin{gathered}
\mu_{Y}(t, x, y, \hat{\nu}(t, x, y, D \tilde{\varphi}(t, x)))-\mathcal{L}^{\hat{\nu}(t, x, y, D \tilde{\varphi}(t, x))} \tilde{\varphi}(t, x) \geq \eta \text { for } \\
(t, x) \in[T-\varepsilon, T] \times \bar{B}_{\varepsilon}\left(x_{0}\right) \text { and } y \in \mathbb{R} \text { such that }|y-\tilde{\varphi}(t, x)| \leq \varepsilon
\end{gathered}
$$

Here we may need to shrink $\varepsilon>0$ again. Since $u^{+}$is USC and $\tilde{\varphi}\left(T, x_{0}\right)=u^{+}\left(T-, x_{0}\right)$, there exists $\alpha>0$ such that $\tilde{\varphi}>u^{+}-2 \alpha$ on $\left.[T-\varepsilon, T) \times \bar{B}_{\varepsilon / 2}\left(x_{0}\right)\right)$ after possibly 
shrinking $\varepsilon$ another time. Since $w_{k} \searrow u^{+}$, there exists $n_{0} \in \mathbb{N}$ such that

$$
\tilde{\varphi}>w_{n_{0}}-\alpha \text { on }[T-\varepsilon, T) \times \bar{B}_{\varepsilon / 2}\left(x_{0}\right) .
$$

Since $\min _{0 \leq t \leq T}\left(\tilde{\varphi}(t, x)-w_{1}(t, x)\right) \rightarrow \infty$ as $|x| \rightarrow \infty$, we can find $R_{0}>\varepsilon$ such that

$$
\tilde{\varphi}>w_{n_{0}}+\varepsilon \text { on } \mathbb{O}:=[T-\varepsilon, T] \times\left(\mathbb{R}^{d}-\bar{B}_{R_{0}}\left(x_{0}\right)\right) \text {. }
$$

Notice that $\tilde{\varphi}(T, \cdot)-u^{+}(T-, \cdot)$ is strictly positive on the compact set $\mathbb{T}^{*}:=\bar{B}_{R_{0}}\left(x_{0}\right)$ $B_{\varepsilon / 2}\left(x_{0}\right)$. Hence, by the upper semi-continuity of $u^{+}(T-, \cdot)$, there exists $\zeta>0$ such that

$$
\tilde{\varphi}(T, \cdot)>u^{+}(T-, \cdot)+4 \zeta \text { on } \mathbb{T}^{*}
$$

From (2.4.8), we conclude that there exists $\sigma>0$ such that

$$
\tilde{\varphi}>u^{+}+2 \zeta \text { on }[T-\sigma, T) \times \mathbb{T}^{*}
$$

More precisely, if (2.4.9) does not hold for any $\sigma>0$, then there exists a sequence $\left(t_{n}, x_{n}\right) \in \mathbb{D}_{i}$ such that $t_{n} \rightarrow T, x_{n} \in \mathbb{T}^{*}$ and $\tilde{\varphi}\left(t_{n}, x_{n}\right) \leq u^{+}\left(t_{n}, x_{n}\right)+2 \zeta$. The compactness of $\mathbb{T}^{*}$ implies that there is a subsequence of $\left(t_{n}, x_{n}\right)$ which converges to $\left(T, x^{\prime}\right)$ for some $x^{\prime} \in \mathbb{T}^{*}$. By taking the limsup of the above equation over the subsequence, we get $\tilde{\varphi}\left(T, x^{\prime}\right) \leq u^{+}\left(T-, x^{\prime}\right)+2 \zeta$. This contradicts (2.4.8). Therefore, (2.4.9) holds.

In (2.4.9), we choose $\sigma<\varepsilon$. By a Dini-type argument, there exists $n_{1} \geq n_{0}$ such that

$$
\tilde{\varphi}>w_{n_{1}}+\zeta \text { on }[T-\sigma, T) \times \mathbb{T}^{*}
$$

Set $w=w_{n_{1}}$. For $\kappa \in(0, \varepsilon \wedge \alpha \wedge \zeta)$, define

$$
w^{\kappa}:=\left\{\begin{array}{l}
(\tilde{\varphi}-\kappa) \wedge w \text { on }[T-\sigma, T] \times \bar{B}_{\varepsilon}\left(x_{0}\right), \\
w \text { outside }[T-\sigma, T] \times \bar{B}_{\varepsilon}\left(x_{0}\right) .
\end{array}\right.
$$


Since $w(T, x) \geq g(x)$ and (2.4.2) holds, we get that $w^{\kappa}(T, x) \geq g(x)$ for all $x \in \mathbb{R}^{d}$. We also notice that

$$
w^{\kappa}\left(T, x_{0}\right) \leq \varphi\left(x_{0}\right)-\kappa<u^{+}\left(T-, x_{0}\right) \leq u^{+}\left(T, x_{0}\right)
$$

Using (2.4.4), (2.4.5), (2.4.6), (2.4.7) and (2.4.10) in a manner that is similar to Step 1 in Theorem 2.3.3's proof, we can show that $w^{\kappa}$ is a stochastic super-solution, which contradicts (2.4.11).

Step 2 (The super-solution property on $\mathbb{D}_{T}$ ). We will divide the proof into two steps:

Step 2.A. We will show that $u^{-}(T-, \cdot)$ is a viscosity super-solution of

$$
(\varphi(x)-g(x)) \mathbb{1}_{\left\{H^{*} \varphi(x)<\infty\right\}} \geq 0 \text { on } \mathbb{R}^{d}
$$

Let $x_{0} \in \mathbb{R}^{d}$ and $\varphi \in C^{2}\left(\mathbb{R}^{d}\right)$ be such that

$$
0=\left(u^{-}\left(T-, x_{0}\right)-\varphi\left(x_{0}\right)\right)=\min _{x \in \mathbb{R}^{d}}\left(u^{-}(T-, x)-\varphi(x)\right) .
$$

Assuming that $H^{*} \varphi\left(x_{0}\right)=C<\infty$ and that $g\left(x_{0}\right)>u^{-}\left(T-, x_{0}\right)=\varphi\left(x_{0}\right)$, we will work towards a contradiction. Let $\left\{w_{k}\right\}_{1}^{\infty}$ be a sequence in $\mathbb{U}^{-}$such that $w_{n} \nearrow u^{-}$. Let $\tilde{\varphi}(t, x)=\varphi(x)-\iota\left|x-x_{0}\right|^{n_{0}}-(C+2)(T-t)$ and $\tilde{\varphi}^{\prime}(x)=\varphi(x)-\iota\left|x-x_{0}\right|^{n_{0}}$ for $\iota>0$, where $\iota$ will be fixed later. $n_{0} \geq 2$ is chosen such that for any $\iota>0$,

$$
\max _{0 \leq t \leq T}\left(\tilde{\varphi}(t, x)-w_{1}(t, x)\right) \rightarrow-\infty, \max _{0 \leq t \leq T} \tilde{\varphi}(t, x) \rightarrow-\infty \text { as }|x| \rightarrow \infty
$$

Note that $D \tilde{\varphi}^{\prime}(x)=D \tilde{\varphi}(t, x)$ and $D^{2} \tilde{\varphi}^{\prime}(x)=D^{2} \tilde{\varphi}(t, x)$. From $g\left(x_{0}\right)>\varphi\left(x_{0}\right)=$ $\tilde{\varphi}\left(T, x_{0}\right)=u^{-}\left(T-, x_{0}\right)$ and the lower semi-continuity of $g$ and $u^{-}$, we can find $\varepsilon>0$ and $\eta \in(0,1)$ such that

$$
\begin{aligned}
& g(x)-\tilde{\varphi}(t, x)>\varepsilon \text { for }(t, x) \in \bar{B}_{\varepsilon}\left(T, x_{0}\right), \\
& \tilde{\varphi}<u^{-}+2 \eta \text { on }[T-\varepsilon, T) \times \bar{B}_{\varepsilon / 2}\left(x_{0}\right) .
\end{aligned}
$$


By the locally boundedness of $\mu_{X}, \sigma_{X}, \mu_{Y}, b$ and $\beta$, and $H^{*} \varphi\left(x_{0}\right)=C$, there exists $\iota>0$ such that

$$
\begin{gathered}
\mu_{Y}(t, x, y, u)-\mu_{X}^{\top}(t, x, u) D \tilde{\varphi}(t, x)-\frac{1}{2} \operatorname{Tr}\left[\sigma_{X} \sigma_{X}^{\top}(t, x, u) D^{2} \tilde{\varphi}(t, x)\right] \leq C+1 \text { for all } \\
(t, x) \in[T-\varepsilon, T] \times \bar{B}_{\varepsilon}\left(x_{0}\right),(y, u) \in \mathbb{R} \times \mathbb{R} \text { such that }|y-\tilde{\varphi}(t, x)| \leq \varepsilon \text { and } \\
u \in \mathcal{N}_{\varepsilon,-\eta}\left(t, x, y, D \tilde{\varphi}(t, x), \tilde{\varphi}^{\prime}\right)
\end{gathered}
$$

Here, we may need to choose smaller values of $\varepsilon$ and $\eta$. Therefore, by the definition of $\Delta^{u, e}$,

$$
\begin{aligned}
& \mu_{Y}(t, x, y, u)-\mathscr{L}^{u} \tilde{\varphi}(t, x) \leq C+1-C-2 \leq-\eta \text { for }(t, x, y) \in \mathbb{D} \times \mathbb{R} \times U \text { s.t. } \\
& (t, x) \in[T-\varepsilon, T] \times \bar{B}_{\varepsilon}\left(x_{0}\right),|y-\tilde{\varphi}(t, x)| \leq \varepsilon \text { and } u \in \mathcal{N}_{\varepsilon,-\eta}(t, x, y, D \tilde{\varphi}(t, x), \tilde{\varphi}) .
\end{aligned}
$$

Fix $\iota$. Since $w_{k} \nearrow u^{-}$, there exists $n_{0} \in \mathbb{N}$ such that

$$
\tilde{\varphi}<w_{n_{0}}+\eta \text { on }[T-\varepsilon, T) \times \bar{B}_{\varepsilon / 2}\left(x_{0}\right)
$$

due to (2.4.13). By (2.4.12), there exists $R_{0}>\varepsilon$ such that

$$
\tilde{\varphi}(t, x)<w_{n_{0}}(t, x)+\varepsilon \leq w_{n}(t, x)+\varepsilon \text { on } \mathbb{O} \text { for } n \geq n_{0},
$$

where $\mathbb{O}:=[T-\varepsilon, T] \times\left(\mathbb{R}^{d}-\bar{B}_{R_{0}}\left(x_{0}\right)\right)$. Since $\tilde{\varphi}(T, x) \leq \varphi(x), u^{-}(T-, \cdot)-\tilde{\varphi}(T, \cdot)$ is strictly positive on the compact set $\mathbb{T}^{*}:=\bar{B}_{R_{0}}\left(x_{0}\right)-B_{\varepsilon / 2}\left(x_{0}\right)$. Hence, by the lower semi-continuity of $u^{-}(T-, \cdot)$, there exists $\alpha>0$ such that

$$
\tilde{\varphi}(T, \cdot)<u^{-}(T-, \cdot)-4 \alpha \text { on } \mathbb{T}^{*}
$$

Similar to Step 1 in this proof, we can find $\sigma \in(0, \varepsilon)$ and $n_{1} \geq n_{0}$ such that $\tilde{\varphi}<w_{n_{1}}-\alpha$ on $[T-\sigma, T) \times \mathbb{T}^{*}$. Set $w=w_{n_{1}}$. For $\kappa \in(0, \varepsilon \wedge \delta \wedge \alpha)$, define

$$
w^{\kappa}:=\left\{\begin{array}{l}
(\tilde{\varphi}+\kappa) \vee w \text { on }[T-\sigma, T] \times \bar{B}_{\varepsilon}\left(x_{0}\right), \\
w \text { outside }[T-\sigma, T] \times \bar{B}_{\varepsilon}\left(x_{0}\right) .
\end{array}\right.
$$


As in Step 2 of Theorem 2.3.3's proof, we can show that $w^{\kappa} \in \mathbb{U}^{-}$, which yields a contradiction.

Step 2.B: In this step, we prove that $u^{-}(T-, \cdot)$ is a viscosity super-solution of $\delta^{*} \varphi(x) \geq 0$. Let $x_{0} \in \mathbb{R}^{d}$ and $\varphi \in C^{2}\left(\mathbb{R}^{d}\right)$ be such that

$$
0=\left(u^{-}\left(T-, x_{0}\right)-\varphi\left(x_{0}\right)\right)=\min _{R^{d}}\left(u^{-}(T-, x)-\varphi(x)\right) .
$$

Let $\left(s_{n}, \xi_{n}\right)$ be a sequence in $\mathbb{D}_{i}$ satisfying $\left(s_{n}, \xi_{n}\right) \rightarrow\left(T, x_{0}\right)$ and $u^{-}\left(s_{n}, \xi_{n}\right) \rightarrow$ $u^{-}\left(T-, x_{0}\right)=\varphi\left(x_{0}\right)$. For all $n \in \mathbb{N}, k \geq 0$ and $\iota \geq 0$, define

$$
\varphi_{n}^{k, \iota}(t, x)=\varphi(x)-\iota\left|x-x_{0}\right|^{4}+k \frac{T-t}{\left(T-s_{n}\right)}, \varphi^{\iota}(x)=\varphi(x)-\iota\left|x-x_{0}\right|^{4} .
$$

Notice that

$$
\lim _{\iota \rightarrow 0} \lim _{k \rightarrow 0} \limsup _{n \rightarrow \infty} \sup _{(t, x) \in\left[s_{n}, T\right] \times \bar{B}_{1}\left(x_{0}\right)}\left|\varphi_{n}^{k, \iota}(t, x)-\varphi(x)\right|=0 .
$$

Let $\left(t_{n}^{k, \iota}, x_{n}^{k, \iota}\right)$ be the minimizer of $u^{-}-\varphi_{n}^{k, \iota}$ on $\left[s_{n}, T\right] \times \bar{B}_{1}\left(x_{0}\right)$. We claim that for any $k>0$ and $\iota>0$, there exists $N^{k, \iota} \in \mathbb{N}$ such that

$$
s_{n} \leq t_{n}^{k, \iota}<T \text { for all } n \geq N^{k, \iota} \text {, and } x_{n}^{k, \iota} \rightarrow x_{0} \text { as } n \rightarrow \infty
$$

We now prove (2.4.14). Since $\left(s_{n}, \xi_{n}\right) \rightarrow\left(T, x_{0}\right)$, we can find $N^{k, \iota} \in \mathbb{N}$ such that for $n \geq N^{k, \iota}$

$$
\left(u^{-}-\varphi_{n}^{k, \iota}\right)\left(s_{n}, \xi_{n}\right)=u^{-}\left(s_{n}, \xi_{n}\right)-\varphi\left(\xi_{n}\right)+\iota\left|\xi_{n}-x_{0}\right|^{4}-\frac{1}{k} \leq-\frac{1}{2 k}<0 .
$$

On the other hand,

$$
\liminf _{t \uparrow T, x^{\prime} \rightarrow x}\left(u^{-}-\varphi_{n}^{k, \iota}\right)\left(t, x^{\prime}\right)=u^{-}(T-, x)-\varphi(x)+\iota\left|x-x_{0}\right|^{4} \geq 0 \text { for }\left|x-x_{0}\right| \leq 1 .
$$

By (2.4.15) and (2.4.16), the first part of (2.4.14) holds. By an argument similar to Step 4 in Theorem 3.1's proof in [9], we know that the second part of (2.4.14) also holds. From (2.4.14) and the definition of $\varphi_{n}^{k, \iota}$, we also see that

$$
\varphi_{n}^{k, \iota}\left(t_{n}^{k, \iota}, x_{n}^{k, \iota}\right) \rightarrow u^{-}\left(T-, x_{0}\right)=\varphi\left(x_{0}\right) \text { as } n \rightarrow \infty, \text { then } k \rightarrow 0, \iota \rightarrow 0
$$


By (2.4.14), (2.4.17) and the facts that $\liminf _{(t<T, x) \rightarrow\left(T, x_{0}\right)} u^{-}(t, x)=u^{-}\left(T-, x_{0}\right)$ and $u^{-}\left(t_{n}^{k, \iota}, x_{n}^{k, \iota}\right) \leq \varphi_{n}^{k, \iota}\left(t_{n}^{k, \iota}, x_{n}^{k, \iota}\right)$, it holds that $u^{-}\left(t_{n}^{k, \iota}, x_{n}^{k, \iota}\right) \rightarrow u^{-}\left(T-, x_{0}\right)=\varphi\left(x_{0}\right)$ as $n \rightarrow \infty$ then $k \rightarrow 0, \iota \rightarrow 0$. Since for all $k>0, \iota>0$ and $n \geq N^{k, \iota},\left(t_{n}^{k, \iota}, x_{n}^{k, \iota}\right)$ is a local minimizer of $u^{-}-\varphi_{n}^{k, \iota}$ and $t_{n}^{k, \iota}<T$, we get

$$
-\partial_{t} \varphi_{n}^{k, \iota}\left(t_{n}^{k, \iota}, x_{n}^{k, \iota}\right)+H^{*}\left(t_{n}^{k, \iota}, x_{n}^{k, \iota}, u^{-}\left(t_{n}^{k, \iota}, x_{n}^{k, \iota}\right), D \varphi_{n}^{k, \iota}\left(t_{n}^{k, \iota}, x_{n}^{k, \iota}\right), D^{2} \varphi_{n}^{k, \iota}\left(t_{n}^{k, \iota}, x_{n}^{k, \iota}\right)\right) \geq 0
$$

from Theorem 2.3.3. By the definition of $H^{*}$, for any $k>0, \iota>0$ and $n \geq N_{n}^{k, \iota}$, there exists a sequence $\left\{\left(\varepsilon_{m}, \eta_{m}, \Theta_{m}, \varphi_{m}\right)\right\} \subset \mathbb{R}_{+} \times[-1,1] \times \mathbb{D} \times \mathbb{R} \times \mathbb{R}^{d} \times \mathbb{M}^{d} \times C(\mathbb{D})^{7}$ such that $\left(\varepsilon_{m}, \eta_{m}\right) \rightarrow(0,0)$,

$$
\begin{aligned}
\varphi_{m} \stackrel{\text { u.c. }}{\longrightarrow} & \varphi_{n}^{k, \iota}, \Theta_{m} \rightarrow\left(\cdot, u^{-}(\cdot), D \varphi_{n}^{k, \iota}(\cdot), D^{2} \varphi_{n}^{k, \iota}(\cdot)\right)\left(t_{n}^{k, \iota}, x_{n}^{k, \iota}\right) \text { and } \\
H_{\varepsilon_{m}, \eta_{m}}\left(\Theta_{m}, \varphi_{m}\right) & \rightarrow H^{*}\left(\cdot, u^{-}(\cdot), D \varphi_{n}^{k, \iota}(\cdot), D^{2} \varphi_{n}^{k, \iota}(\cdot), \varphi_{n}^{k, \iota}\right)\left(t_{n}^{k, \iota}, x_{n}^{k, \iota}\right)>-\infty .
\end{aligned}
$$

This implies that $\mathcal{N}_{\varepsilon_{m}, \eta_{m}}\left(t_{m}, x_{m}, y_{m}, p_{m}, \varphi_{m}\right) \neq \emptyset$ since $\sup \emptyset=-\infty$. By the definition of $\delta$, it holds that $\delta\left(t_{m}, x_{m}, y_{m}, p_{m}, \varphi_{m}\right) \geq-\sqrt{\varepsilon_{m}^{2}+\eta_{m}^{2}}$. From (2.4.18) and the definition of $\delta^{*}$, we get

$$
\delta^{*}\left(t_{n}^{k, \iota}, x_{n}^{k, \iota}, u^{-}\left(t_{n}^{k, \iota}, x_{n}^{k, \iota}\right), D \varphi_{n}^{k, \iota}\left(t_{n}^{k, \iota}, x_{n}^{k, \iota}\right), \varphi_{n}^{k, \iota}\right) \geq \limsup _{m \rightarrow \infty} \delta\left(t_{m}, x_{m}, y_{m}, p_{m}, \varphi_{m}\right) \geq 0
$$

By the definition of $\Delta^{u, e}$ in the set-valued map $\mathbf{N}$, the equation above implies that

$$
\delta^{*}\left(\cdot, u^{-}(\cdot), D \varphi_{n}^{k, \iota}(\cdot), \varphi^{\iota}\right)\left(t_{n}^{k, \iota}, x_{n}^{k, \iota}\right)=\delta^{*}\left(\cdot, u^{-}(\cdot), D \varphi_{n}^{k, \iota}(\cdot), \varphi_{n}^{k, \iota}\right)\left(t_{n}^{k, \iota}, x_{n}^{k, \iota}\right) \geq 0
$$

Note that $\varphi^{\iota} \stackrel{\text { u.c. }}{\longrightarrow} \varphi$ as $\iota \rightarrow 0$. Moreover, for $\iota>0, u^{-}\left(t_{n}^{k, \iota}, x_{n}^{k, \iota}\right) \rightarrow \varphi\left(x_{0}\right)$ and $D \varphi_{n}^{k, \iota}\left(t_{n}^{k, \iota}, x_{n}^{k, \iota}\right) \rightarrow D \varphi\left(x_{0}\right)$ as $n \rightarrow \infty$ then $k \rightarrow 0$. Taking the limsup of (2.4.19) by first sending $n \rightarrow \infty$ then $k \rightarrow 0$ and $\iota \rightarrow 0$, we have

$$
\delta^{*} \varphi\left(x_{0}\right)=\delta^{*} \varphi\left(T, x_{0}, \varphi\left(x_{0}\right), D \varphi\left(x_{0}\right), \varphi\right) \geq 0
$$

from the upper semi-continuity of $\delta^{*}$,

\footnotetext{
${ }^{7} \Theta_{m}:=\left(t_{m}, x_{m}, y_{m}, p_{m}, A_{m}\right)$ takes value in $\mathbb{D} \times \mathbb{R} \times \mathbb{R}^{d} \times \mathbb{M}^{d}$
} 


\subsection{Verification by comparison}

We now carry out the verification for non-smooth functions assuming the comparison principle as in [9].

Assumption 2.5.1. Let $H=H_{*}$. Assume that $H=H^{*}$ on the set $\{H<\infty\}$ and that there exists an LSC function $G: \mathbb{D} \times \times \mathbb{R} \times \mathbb{R}^{d} \times \mathbb{M}^{d} \times C(\mathbb{D}) \rightarrow \mathbb{R}$ such that

$$
\begin{aligned}
& \text { (a) } H(t, x, y, p, A, \varphi)<\infty \Longrightarrow G(t, x, y, p, A, \varphi) \leq 0 \\
& \text { (b) } G(t, x, y, p, A, \varphi)<0 \Longrightarrow H(t, x, y, p, A, \varphi)<\infty
\end{aligned}
$$

Proposition 2.5.2. Under Assumptions 2.2.1, 2.2.2, 2.2.8, 2.2.11 and 2.5.1, $u^{+}$ (resp. $\left.u^{-}\right)$is a USC (resp. an LSC) viscosity sub-solution (resp. super-solution) of

$$
\max \left\{-\partial_{t} \varphi(t, x)+H \varphi(t, x), G \varphi(t, x)\right\}=0 \text { on } \mathbb{D}_{i}
$$

Moreover, if $g$ is $U S C, u^{+}(T-, \cdot)$ is a USC viscosity sub-solution of

$$
\min \left\{\max \{\varphi(x)-g(x), G \varphi(x)\}, \delta_{*} \varphi(x)\right\} \leq 0 \text { on } \mathbb{R}^{d}
$$

If $g$ is $L S C, u^{-}(T-, \cdot)$ is an LSC viscosity super-solution of

$$
\min \left\{\max \{\varphi(x)-g(x), G \varphi(x)\}, \delta^{*} \varphi(x)\right\} \geq 0 \text { on } \mathbb{R}^{d}
$$

Proof (1) The sub-solution property in $\mathbb{D}_{i}$. Suppose

$$
0=\left(u^{+}-\varphi\right)\left(t_{0}, x_{0}\right)=\max _{\mathbb{D}_{i}}\left(u^{+}-\varphi\right) \text { for some }\left(t_{0}, x_{0}\right) \in \mathbb{D}_{i} \text { and } \varphi \in C^{1,2}(\mathbb{D})
$$

Then $-\partial_{t} \varphi\left(t_{0}, x_{0}\right)+H \varphi\left(t_{0}, x_{0}\right)=-\partial_{t} \varphi\left(t_{0}, x_{0}\right)+H_{*} \varphi\left(t_{0}, x_{0}\right) \leq 0$ from Theorem 2.3.3. From (a) in Assumption 2.5.1, $G \varphi\left(t_{0}, x_{0}\right) \leq 0$. Therefore, the sub-solution property holds for $u^{+}$in the parabolic interior.

(2) The super-solution property in $\mathbb{D}_{i}$. Suppose

$$
0=\left(u^{-}-\varphi\right)\left(t_{0}, x_{0}\right)=\min _{\mathbb{D}_{i}}\left(u^{-}-\varphi\right) \text { for some }\left(t_{0}, x_{0}\right) \in \mathbb{D}_{i} \text { and } \varphi \in C^{1,2}(\mathbb{D})
$$


If $H \varphi\left(t_{0}, x_{0}\right)<\infty,-\partial_{t} \varphi\left(t_{0}, x_{0}\right)+H \varphi\left(t_{0}, x_{0}\right)=-\partial_{t} \varphi\left(t_{0}, x_{0}\right)+H^{*} \varphi\left(t_{0}, x_{0}\right) \geq 0$ from Assumption 2.5.1 and Theorem 2.3.3. On the other hand, if $H \varphi\left(t_{0}, x_{0}\right)=\infty$, $G \varphi\left(t_{0}, x_{0}\right) \geq 0$ from (b) in Assumption 2.5.1. Therefore, the viscosity super-solution property holds for $u^{-}$in the parabolic interior.

(3) The sub-solution property on $\mathbb{D}_{T}$. From Theorem 2.4.1, we know that $u^{+}(T-, \cdot)$ is viscosity sub-solution of

$$
\min \left\{\varphi(x)-g(x), \delta_{*} \varphi(x)\right\} \leq 0 \text { on } \mathbb{R}^{d}
$$

Therefore, it suffices to show that $G u^{+}(T-, \cdot) \leq 0$ in the viscosity sense. Let $x_{0} \in \mathbb{R}^{d}$ and $\varphi \in C^{2}\left(\mathbb{R}^{d}\right)$ be such that

$$
0=\left(u^{+}\left(T-, x_{0}\right)-\varphi\left(x_{0}\right)\right)=\max _{x \in \mathbb{R}^{d}}\left(u^{+}(T-, x)-\varphi(x)\right) .
$$

Let $\left(s_{n}, \xi_{n}\right)$ be a sequence in $\mathbb{D}_{i}$ satisfying $\left(s_{n}, \xi_{n}\right) \rightarrow\left(T, x_{0}\right)$ and $u^{+}\left(s_{n}, \xi_{n}\right) \rightarrow$ $u^{+}\left(T-, x_{0}\right)$. For all $n \in \mathbb{N}, k \geq 0$ and $\iota \geq 0$, define

$$
\varphi_{n}^{k, \iota}(t, x)=\varphi(x)+\iota\left|x-x_{0}\right|^{4}-k \frac{T-t}{\left(T-s_{n}\right)}, \varphi^{\iota}(x)=\varphi(x)+\iota\left|x-x_{0}\right|^{4}
$$

Let $\left(t_{n}^{k, \iota}, x_{n}^{k, \iota}\right)$ be the maximizer of $u^{+}-\varphi_{n}^{k, \iota}$ on $\left[s_{n}, T\right] \times \bar{B}_{1}\left(x_{0}\right)$. Similar to the arguments in Step 2B of Theorem 2.4.1's proof, we can show that

$$
\lim _{k \rightarrow 0, \iota \rightarrow 0} \lim _{n \rightarrow \infty} u^{+}\left(t_{n}^{k, \iota}, x_{n}^{k, \iota}\right)=\varphi\left(x_{0}\right)
$$

We also know that for any $k>0$ and $\iota>0$, there exists $N^{k, \iota} \in \mathbb{N}$ such that $s_{n} \leq t_{n}^{k, \iota}<T$ for all $n \geq N^{k, \iota}$ and $x_{n}^{k, \iota} \rightarrow x_{0}$ as $n \rightarrow \infty$. Therefore, for all $k>0$, $\iota>0$ and $n \geq N^{k, \iota},\left(t_{n}^{k, \iota}, x_{n}^{k, \iota}\right)$ is a maximizer of $u^{+}-\varphi_{n}^{k, \iota}$ on $\left[s_{n}, T\right] \times \bar{B}_{1}\left(x_{0}\right)$. From Theorem 2.3.3,

$$
-\partial_{t} \varphi\left(t_{n}^{k, \iota}, x_{n}^{k, \iota}\right)+H_{*}\left(t_{n}^{k, \iota}, x_{n}^{k, \iota}, u^{+}\left(t_{n}^{k, \iota}, x_{n}^{k, \iota}\right), D \varphi_{n}^{k, \iota}\left(t_{n}^{k, \iota}, x_{n}^{k, \iota}\right), D^{2} \varphi_{n}^{k, \iota}\left(t_{n}^{k, \iota}, x_{n}^{k, \iota}\right), \varphi_{n}^{k, \iota}\right) \leq 0
$$


Hence, the $H_{*}$-term in the above equation is less than $\infty$. From the definition of $\Delta^{u, e}$, we get

$$
H_{*}\left(t_{n}^{k, \iota}, x_{n}^{k, \iota}, u^{+}\left(t_{n}^{k, \iota}, x_{n}^{k, \iota}\right), D \varphi_{n}^{k, \iota}\left(t_{n}^{k, \iota}, x_{n}^{k, \iota}\right), D^{2} \varphi_{n}^{k, \iota}\left(t_{n}^{k, \iota}, x_{n}^{k, \iota}\right), \varphi^{\iota}\right)<\infty
$$

which further implies by Assumption 2.5.1 that

$$
G \varphi\left(t_{n}^{k, \iota}, x_{n}^{k, \iota}, u^{+}\left(t_{n}^{k, \iota}, x_{n}^{k, \iota}\right), D \varphi_{n}^{k, \iota}\left(t_{n}^{k, \iota}, x_{n}^{k, \iota}\right), D^{2} \varphi_{n}^{k, \iota}\left(t_{n}^{k, \iota}, x_{n}^{k, \iota}\right), \varphi^{\iota}\right) \leq 0 .
$$

Using an argument similar to that in Step 2B of Theorem 2.4.1's proof, we conclude that $G \varphi\left(x_{0}\right) \leq 0$.

(4) The super-solution property on $\mathbb{D}_{T}$. It suffices to show that $u^{-}(T-, \cdot)$ is a viscosity super-solution of

$$
\max \{\varphi(x)-g(x), G \varphi(x)\} \geq 0 \text { on } \mathbb{R}^{d} .
$$

Let $x_{0} \in \mathbb{R}^{d}$ and $\varphi \in C^{2}\left(\mathbb{R}^{d}\right)$ be such that

$$
0=\left(u^{-}\left(T-, x_{0}\right)-\varphi\left(x_{0}\right)\right)=\min _{x \in \mathbb{R}^{d}}\left(u^{-}(T-, x)-\varphi(x)\right) .
$$

From Theorem 2.4.1, one of the following two scenarios must hold:

$$
\begin{gathered}
\varphi\left(x_{0}\right) \geq g\left(x_{0}\right), H^{*} \varphi\left(x_{0}\right)<\infty \text { or } \\
H^{*} \varphi\left(x_{0}\right)=\infty .
\end{gathered}
$$

(2.5.2) implies (2.5.1); on the other hand, if (2.5.3) holds, then $H \varphi\left(x_{0}\right)=\infty$, which means that $G \varphi\left(x_{0}\right) \geq 0$ from (b) in Assumption 2.5.1. Therefore, (2.5.1) holds.

Assumption 2.5.3. Assume that $\delta^{*}=\delta_{*}, g$ is continuous and a comparison principle holds between USC sub-solutions and LSC super-solutions for

$$
\min \{\max \{\varphi(x)-g(x), G \varphi(x)\}, \delta \varphi(x)\}=0 \text { on } \mathbb{R}^{d}
$$


In the presence of jumps, it is nontrivial to check this assumption. When there are no jumps in the controlled processes, the comparison principle can be proved in certain classes of functions (see the discussion above Assumption 2.2 in [14]). Also, in Section 2.6, $\delta$ drops out in the corresponding PDE and there are comparison results available for fully non-linear equations with jumps (see [1]).

Lemma 2.5.4. Under Assumptions 2.2.1, 2.2.2, 2.2.8, 2.2.11, 2.5.1 and 2.5.3,

$$
u^{-}(T-, \cdot)=u^{+}(T-, \cdot)=\hat{g}(\cdot)
$$

where $\hat{g}$ is the unique continuous viscosity solution to (2.5.4).

Proof It follows from their definitions that $u^{-} \leq u^{+}$. Since $u^{+}$is USC and $u^{-}$is LSC, then

$$
u^{-}(T-, x)=\liminf _{\left(t<T, x^{\prime}\right) \rightarrow(T, x)} u^{-}\left(t, x^{\prime}\right) \leq \limsup _{\left(t<T, x^{\prime}\right) \rightarrow(T, x)} u^{+}\left(t, x^{\prime}\right)=u^{+}(T-, x) .
$$

Moreover, $u^{+}(T-, \cdot)$ is a viscosity sub-solution and $u^{-}(T-, \cdot)$ is a viscosity supersolution to (2.5.4) due to Theorem 2.4.1. Therefore, the claim holds by Assumption 2.5.3.

Theorem 2.5.5. Suppose that there is a comparison principle for

$$
\max \left\{-\partial_{t} \varphi(t, x)+H \varphi(t, x), G \varphi(t, x)\right\}=0 \text { on } \mathbb{D}_{i}
$$

and that Assumptions 2.2.1, 2.2.2, 2.2.8, 2.2.11, 2.5.1 and 2.5.3 hold. Then there exists a unique continuous viscosity solution $V$ to (2.5.5) with terminal condition $V(T, \cdot)=\hat{g}(\cdot)$ and $u(t, x)=u^{-}(t, x)=u^{+}(t, x)=V(t, x)$ for $(t, x) \in \mathbb{D}_{i}$.

Proof Define

$$
\hat{u}^{+}(t, x):= \begin{cases}u^{+}(t, x), & (t, x) \in \mathbb{D}_{i} \\ \hat{g}(x), & t=T, x \in \mathbb{R}^{d}\end{cases}
$$


and

$$
\hat{u}^{-}(t, x):= \begin{cases}u^{-}(t, x), & (t, x) \in \mathbb{D}_{i}, \\ \hat{g}(x), & t=T, x \in \mathbb{R}^{d} .\end{cases}
$$

From Proposition 2.5.2, $\hat{u}^{-}$is an LSC viscosity super-solution and $\hat{u}^{+}$is a USC viscosity sub-solution of $(2.5 .5)$. Since $\hat{u}^{+}(T, \cdot)=\hat{u}^{-}(T, \cdot), \hat{u}^{+} \leq \hat{u}^{-}$on $\mathbb{D}$ by comparison. Hence, $\hat{u}^{+}=\hat{u}^{-}$on $\mathbb{D}$ from (2.2.7). Define $V:=\hat{u}^{+}=\hat{u}^{-}$. It is a continuous viscosity solution of (2.5.5) satisfying $V(T, x)=\hat{g}(x)$. Uniqueness follows directly from the comparison principle.

\subsection{Stochastic control as a stochastic target problem}

In this section, we show how the HJB equation associated to an optimal control problem in standard form can be deduced from a stochastic target problem. Given a bounded continuous function $g: \mathbb{R}^{d} \rightarrow \mathbb{R}$, we define an optimal control problem by

$$
\mathbf{u}(t, x):=\inf _{\nu \in \mathcal{U}^{t}} \mathbb{E}\left[g\left(X_{t, x}^{\nu}(T)\right)\right]
$$

We follow the setup of Section 2.2 with one exception: $\mathcal{U}^{t}$ is the collection of all $\mathbb{F}^{t}$-predictable processes in $\mathbb{L}^{2}\left(\Omega \times[0, T], \mathcal{F} \otimes \mathcal{B}[0, T], \mathbb{P} \otimes \lambda_{L} ; U\right)$, where $U \subset \mathbb{R}^{d}$ and $X$ follows the SDE

$d X(s)=\mu_{X}(s, X(s), \nu(s)) d s+\sigma_{X}(s, X(s), \nu(s)) d W_{s}+\int_{E} \beta(s, X(s-), \nu(s), e) \lambda(d s, d e)$.

To convert the control problem to its stochastic target counterpart, we need the following lemma, which is an adaptation of a result in [13].

Lemma 2.6.1. Suppose Assumptions 2.2.1 and 2.2.2 hold. Define a stochastic target problem as follows:

$$
\begin{gathered}
u(t, x):=\inf \left\{y \in \mathbb{R}: \exists(\nu, \alpha, \gamma) \in \mathcal{U}^{t} \times \mathcal{A}^{t} \times \Gamma^{t} \text { s.t. } Y_{t, y}^{\alpha, \gamma}(T) \geq g\left(X_{t, x}^{\nu}(T)\right)\right\}, \text { where } \\
Y_{t, y}^{\alpha, \gamma}(\cdot):=y+\int_{t} \alpha^{\top}(s) d W_{s}+\int_{t} \int_{E} \gamma^{\top}(s, e) \tilde{\lambda}(d s, d e)
\end{gathered}
$$


and $\mathcal{A}^{t}$ and $\Gamma^{t}$ are the collections of $\mathbb{R}^{d}$-valued and $\mathbb{L}^{2}\left(E, \mathcal{E}, \hat{m} ; \mathbb{R}^{I}\right)$-valued processes, respectively, satisfying the admissibility conditions in Section 2.2. Then $u=\mathbf{u}$ on $\mathbb{D}$.

Proof Since $\mathcal{A}^{t}$ and $\Gamma^{t}$ satisfy the admissibility conditions, this stochastic target problem is well defined. In view of Lemma 2.1 in [13], it suffices to check that

$$
\left\{g\left(X_{t, x}^{\nu}(T), \nu \in \mathcal{U}^{t}\right\} \subset\{M(T), M \in \mathcal{M}\},\right.
$$

$$
\text { where } \mathcal{M}:=\left\{Y_{t, y}^{\alpha, \gamma}(\cdot): y \in \mathbb{R}, \alpha \in \mathcal{A}^{t}, \gamma \in \Gamma^{t}\right\} \text {. }
$$

In fact, by the martingale representation theorem, for any $\nu \in \mathcal{U}^{t}, \mathbb{E}\left[g\left(X_{t, x}^{\nu}(T)\right) \mid \mathcal{F}^{t}\right]$ can be represented in the form of $Y_{t, y}^{\alpha, \gamma}$ for some $\alpha \in \mathcal{A}^{t}$ and $\gamma \in \Gamma_{0}^{t}$, where $\Gamma_{0}^{t}$ is the collection of $\mathbb{L}^{2}\left(E, \mathcal{E}, \hat{m} ; \mathbb{R}^{I}\right)$-valued processes satisfying all of the admissibility conditions except for (2.2.2). In particular, $g\left(X_{t, x}^{\nu}(T)\right)=Y_{t, y}^{\alpha, \gamma}(T)$. Assume, contrary to (2.6.1), that there exists $\nu_{0} \in \mathcal{U}^{t}$ such that

$$
\mathbb{E}\left[g\left(X_{t, x}^{\nu_{0}}(T)\right) \mid \mathcal{F}^{t}\right]=y+\int_{t}^{\cdot} \alpha_{0}^{\top}(s) d W_{s}+\int_{t}^{\cdot} \int_{E} \gamma_{0}^{\top}(s, e) \tilde{\lambda}(d s, d e)
$$

for some $y \in \mathbb{R}, \alpha_{0} \in \mathcal{A}^{t}$ and $\gamma_{0} \in \Gamma_{0}^{t}$, but (2.2.2) does not hold. In the equation above, $\mathbb{E}\left[g\left(X_{t, x}^{\nu_{0}}(T)\right) \mid \mathcal{F}^{t}\right]$ can be chosen to be càdlàg, thanks to Theorem 1.3.13 in [20]. Then for $K>2\|g\|_{\infty}$, there exists $\tau_{0} \in \mathcal{T}_{t}$ such that

$$
\mathbb{P}\left(\left|\int_{E} \gamma^{\top}\left(\tau_{0}, e\right) \lambda\left(\left\{\tau_{0}\right\}, d e\right)\right|>K\right)>0
$$

Suppose that

$$
\mathbb{P}\left(\int_{E} \gamma^{\top}\left(\tau_{0}, e\right) \lambda\left(\left\{\tau_{0}\right\}, d e\right)>K\right)>0 .{ }^{8}
$$

Let $M_{0}(\cdot)=\mathbb{E}\left[g\left(X_{t, x}^{\nu_{0}}(T)\right) \mid \mathcal{F}^{t}\right]$. Therefore,

$$
M_{0}\left(\tau_{0}\right)-M_{0}\left(\tau_{0}-\right)=\int_{E} \gamma^{\top}\left(\tau_{0}, e\right) \lambda\left(\left\{\tau_{0}\right\}, d e\right)>K \text { with positive probability }
$$

\footnotetext{
${ }^{8}$ If this does not hold, the integral is less than $-K$ with positive probability. Noticing this, we can carry out the proof in a similar manner when this assumption does not hold.
} 
Since $\left|M_{0}\right|$ is bounded by $\|g\|_{\infty}<K / 2$, we obtain a contradiction.

Let $\mathbf{H}^{*}$ be the USC envelope of the map $\mathbf{H}: \mathbb{D} \times \mathbb{R}^{d} \times \mathbb{M}^{d} \times C(\mathbb{D}) \rightarrow \mathbb{R}$ defined by

$\mathbf{H}:(t, x, p, A, \varphi) \rightarrow \sup _{u \in U}\left\{-I[\varphi](t, x, u)-\mu_{X}^{\top}(t, x, u) p-\frac{1}{2} \operatorname{Tr}\left[\sigma_{X} \sigma_{X}^{\top}(t, x, u) A\right]\right\}$, where $I[\varphi](t, x, u)=\sum_{1 \leq i \leq I} \int_{E}\left(\varphi\left(t, x+\beta_{i}(t, x, u, e)\right)-\varphi(t, x)\right) m_{i}(d e)$.

Theorem 2.6.2. Under Assumptions 2.2.1 and 2.2.2, $u^{+}$is a USC viscosity subsolution of

$$
-\partial_{t} \varphi(t, x)+\mathbf{H} \varphi(t, x) \leq 0 \text { on } \mathbb{D}_{i}
$$

and $u^{+}(T-, x) \leq g(x)$ for all $x \in \mathbb{R}^{d}$. On the other hand, $u^{-}$is an LSC viscosity super-solution of

$$
-\partial_{t} \varphi(t, x)+\mathbf{H}^{*} \varphi(t, x) \geq 0 \text { on } \mathbb{D}_{i}
$$

and $u^{-}(T-, \cdot)$ is an LSC viscosity super-solution of

$$
(\varphi(x)-g(x)) \mathbb{1}_{\left\{\mathbf{H}^{*} \varphi(x)<\infty\right\}} \geq 0 \text { on } \mathbb{R}^{d}
$$

Proof It is easy to check Assumption 2.2.11 for the stochastic target problem. Since $g$ is bounded, we can check that all of the assumptions in the Appendix A are satisfied, which implies that Assumption 2.2.8 holds. From Theorem 2.3.3, $u^{+}$is a USC viscosity sub-solution of $-\partial_{t} \varphi(t, x)+H_{*} \varphi(t, x) \leq 0$ on $\mathbb{D}_{i}$ and $u^{-}$is an LSC viscosity super-solution of $-\partial_{t} \varphi(t, x)+H^{*} \varphi(t, x) \geq 0$ on $\mathbb{D}_{i}$. From Proposition 3.1 in [13], $H^{*} \leq \mathbf{H}^{*}$ and $H_{*} \geq \mathbf{H}$. This implies that the viscosity properties in the parabolic interior hold.

Also, by Theorem 2.4.1, $u^{+}(T-, \cdot)$ is a USC viscosity sub-solution of

$$
\min \left\{\varphi(x)-g(x), \delta_{*} \varphi(x)\right\} \leq 0 \text { on } \mathbb{R}^{d}
$$

and $u^{-}(T-, \cdot)$ is an LSC viscosity super-solution of

$$
\min \left\{(\varphi(x)-g(x)) \mathbb{1}_{\left\{H^{*} \varphi(x)<\infty\right\}}, \delta^{*} \varphi(x)\right\} \geq 0 \text { on } \mathbb{R}^{d}
$$


where $\delta=\operatorname{dist}\left(0, \mathbf{N}^{c}\right)-\operatorname{dist}(0, \mathbf{N})$ and

$$
\begin{aligned}
& \mathbf{N}(t, x, y, p, \varphi)= \\
& \left\{(q, s): \exists(u, a, r) \in U \times \mathbb{R}^{d} \times \mathbb{L}^{2}\left(E, \mathcal{E}, \hat{m} ; \mathbb{R}^{I}\right) \text { s.t. } q=a-\sigma_{X}^{\top}(t, x, u) p\right. \\
& \text { and } \left.s \leq \min _{1 \leq i \leq I}\left\{r_{i}(e)-\varphi\left(t, x+\beta_{i}(t, x, u, e)\right)+\varphi(t, x)\right\} \hat{m}-\text { a.s. } e \in E\right\} .
\end{aligned}
$$

Obviously, $\mathbf{N}=\mathbb{R}^{d} \times \mathbb{R}$. Therefore, $\delta=\infty$ and the boundary conditions hold.

The following two corollaries show that $\mathbf{u}$ is the unique viscosity solution to its associated HJB equation. We omit the proof, since it is the same as the proofs of Proposition 2.5.2 and Theorem 2.5.5.

Corollary 2.6.3. Suppose that Assumptions 2.2.1 and 2.2.2 hold, $\mathbf{H}=\mathbf{H}^{*}$ on $\{\mathbf{H}<$ $\infty$ and there exists an LSC function $\mathbf{G}: \mathbb{D} \times \mathbb{R} \times \mathbb{R}^{d} \times \mathbb{M}^{d} \times C(\mathbb{D}) \rightarrow \mathbb{R}$ such that

$$
\begin{aligned}
& \text { (a) } \mathbf{H}(t, x, y, p, M, \varphi)<\infty \Longrightarrow \mathbf{G}(t, x, y, p, M, \varphi) \leq 0, \\
& \text { (b) } \mathbf{G}(t, x, y, p, M, \varphi)<0 \Longrightarrow \mathbf{H}(t, x, y, p, M, \varphi)<\infty .
\end{aligned}
$$

Then $u^{+}$(resp. $\left.u^{-}\right)$is a USC (resp. an LSC) viscosity sub-solution (resp. supersolution) of

$$
\max \left\{-\partial_{t} \varphi(t, x)+\mathbf{H} \varphi(t, x), \mathbf{G} \varphi(t, x)\right\}=0 \text { on } \mathbb{D}_{i}
$$

and $u^{+}(T-, \cdot)$ (resp. $\left.u^{-}(T-, \cdot)\right)$ is a USC (resp. an LSC) viscosity sub-solution (resp. super-solution) of

$$
\max \{\varphi(x)-g(x), \mathbf{G} \varphi(x)\}=0 \text { on } \mathbb{R}^{d}
$$

Corollary 2.6.4. Suppose that all of the assumptions in Corollary 2.6.3 hold. Additionally, assume that there is a comparison principle between USC sub-solutions and LSC super-solutions for the PDE

$$
\max \{\varphi(x)-g(x), \mathbf{G} \varphi(x)\}=0 \text { on } \mathbb{R}^{d}
$$


Then $u^{+}(T-, x)=u^{-}(T-, x)=\hat{\mathbf{g}}(x)$, where $\hat{\mathbf{g}}$ is the unique viscosity solution to (2.6.2). Furthermore, if the comparison principle holds for

$$
\max \left\{-\partial_{t} \varphi(t, x)+\mathbf{H} \varphi(t, x), \mathbf{G} \varphi(t, x)\right\}=0 \text { on } \mathbb{D}_{i},
$$

then there exists a unique continuous viscosity solution $\mathbf{V}$ to (2.6.3) with terminal condition $\mathbf{V}(T, x)=\hat{\mathbf{g}}(x)$ and $\mathbf{u}(t, x)=u(t, x)=u^{+}(t, x)=u^{-}(t, x)=\mathbf{V}(t, x)$ for $(t, x) \in \mathbb{D}_{i}$.

\subsection{Appendix: the nonemptiness of $\mathbb{U}^{+}$and $\mathbb{U}^{-}$.}

Assumption 2.7.1. $g$ is bounded.

Assumption 2.7.2. There exists $u_{0} \in U$ such that

$$
\sigma_{Y}\left(t, x, y, u_{0}\right)=0 \text { and } b\left(t, x, y, u_{0}(e), e\right)=0
$$

for all $(t, x, y, e) \in \mathbb{D} \times \mathbb{R} \times E$.

Remark 2.7.3. In the context of super-hedging in mathematical finance, the assumption above is equivalent to restricting trading to the riskless assets.

Proposition 2.7.4. Under Assumptions 2.2.1, 2.2.2, 2.7.1 and 2.7.2, $\mathbb{U}^{+}$is not empty.

Proof. Step 1. In this step we assume that $\mu_{Y}$ is non-decreasing in its $y$-variable. We will show that $w(t, x)=\gamma-e^{k t}$ is a stochastic super-solution for some choice of $k$ and $\gamma$.

By the linear growth condition on $\mu_{Y}$ in Assumption 2.2.2, there exists $L>0$ such that $\left|\mu_{Y}\left(t, x, y, u_{0}\right)\right| \leq L(1+|y|)$, where $u_{0}$ is the element in $U$ in Assumption 2.7.2. Choose $k \geq 2 L$ and $\gamma$ such that $-e^{k T}+\gamma \geq\|g\|_{\infty}$. Then $w(T, x) \geq g(x)$. It 
suffices to show that for any $(t, x, y) \in \mathbb{D} \times \mathbb{R}, \tau \in \mathcal{T}_{t}, \nu \in \mathcal{U}^{t}$ and $\rho \in \mathcal{T}_{\tau}$,

$$
Y(\rho) \geq w(\rho, X(\rho)) \mathbb{P} \text {-a.s. on }\{Y(\tau) \geq w(\tau, X(\tau))\},
$$

where $X:=X_{t, x}^{\nu \otimes_{\tau} u_{0}}, Y:=Y_{t, x, y}^{\nu \otimes_{\tau} u_{0}}$. Let $A=\{Y(\tau)>w(\tau, X(\tau))\}, V(s)=w(s, X(s))$ and $\Gamma(s)=(V(s)-Y(s)) \mathbb{1}_{A}$. Therefore,

$$
\Gamma(s)=\mathbb{1}_{A} \int_{\tau}^{s}(\xi(q)+\Delta(q)) d q \text { for } s \geq \tau,
$$

where

$$
\begin{aligned}
\Delta(s) & :=-k e^{k s}-\mu_{Y}\left(s, X(s), Y(s), u_{0}\right) \leq-k e^{k s}-\mu_{Y}\left(s, X(s),-e^{k s}, u_{0}\right) \\
& \leq-k e^{k s}+L\left(1+e^{k s}\right) \leq 0, \\
\xi(s) & :=\mu_{Y}\left(s, X(s), V(s), u_{0}\right)-\mu_{Y}\left(s, X(s), Y(s), u_{0}\right) .
\end{aligned}
$$

Therefore, from (2.7.2) it holds that

$$
\Gamma(s) \leq \mathbb{1}_{A} \int_{\tau}^{s} \xi(q) d q \text { and } \Gamma^{+}(s) \leq \mathbb{1}_{A} \int_{\tau}^{s} \xi^{+}(q) d q \text { for } s \geq \tau \text {. }
$$

From the Lipschitz continuity of $\mu_{Y}$ in $y$-variable in Assumption 2.2.2,

$$
\Gamma^{+}(s) \leq \mathbb{1}_{A} \int_{\tau}^{s} \xi^{+}(q) d q \leq \int_{\tau}^{s} L_{0} \Gamma^{+}(q) d q \text { for } s \geq \tau,
$$

where $L_{0}$ is the Lipschitz constant of $\mu_{Y}$ with respect to $y$. Note that we use the assumption that $\mu_{Y}$ is non-decreasing in its $y$-variable to obtain the second inequality. Since $\Gamma^{+}(\tau)=0$, an application of Grönwall's Inequality implies that $\Gamma^{+}(\rho) \leq 0$, which further implies that (2.7.1) holds.

Step 2. We get rid of our assumption on $\mu_{Y}$ from Step 1 by following a proof similar to those in [16]. For $c>0$, define $\widetilde{Y}_{t, x, y}^{\nu}$ as the strong solution of

$$
\begin{aligned}
d \widetilde{Y}(s) & =\tilde{\mu}_{Y}\left(s, X_{t, x}^{\nu}(s), \widetilde{Y}(s), \nu(s)\right) d s+\tilde{\sigma}_{Y}^{\top}\left(s, X_{t, x}^{\nu}(s), \widetilde{Y}(s), \nu(s)\right) d W_{s} \\
& +\int_{E} \widetilde{b}^{\top}\left(s, X_{t, x}^{\nu}(s-), \widetilde{Y}(s-), \nu_{1}(s), \nu_{2}(s, e), e\right) \lambda(d s, d e)
\end{aligned}
$$


with initial data $\tilde{Y}(t)=y$, where

$$
\begin{aligned}
\widetilde{\mu}_{Y}(t, x, y, u) & :=c y+e^{c t} \mu_{Y}\left(t, x, e^{-c t} y, u\right), \\
\widetilde{\sigma}_{Y}(t, x, y, u) & :=e^{c t} \sigma_{Y}\left(t, x, e^{-c t} y, u\right), \\
\widetilde{b}(t, x, y, u(e), e) & :=e^{c t} b\left(t, x, e^{-c t} y, u(e), e\right) .
\end{aligned}
$$

Therefore,

$$
\tilde{Y}_{t, x, y}^{\nu}(s) e^{-c s}=Y_{t, x, y e^{-c t}}^{\nu}(s), t \leq s \leq T
$$

Let $\tilde{u}(t, x)=\inf \left\{y \in \mathbb{R}: \exists \nu \in \mathcal{U}^{t}\right.$, s.t. $\tilde{Y}_{t, x, y}^{\nu}(T) \geq \tilde{g}\left(X_{t, x}^{\nu}(T)\right)$-a.s. $\}$, where $\tilde{g}(x)=$ $e^{c T} g(x)$. Therefore, $\tilde{u}(t, x)=e^{c t} u(t, x)$. Since $\mu_{Y}$ is Lipschitz in $y$, we can choose $c>0$ so that

$$
\widetilde{\mu}_{Y}:(t, x, y, u) \mapsto c y+e^{c t} \mu_{Y}\left(t, x, e^{-c t} y, u\right)
$$

is non-decreasing in $y$. Moreover, all the properties of $\widetilde{\mu}_{Y}, \widetilde{\sigma}_{Y}$ and $\widetilde{b}$ in Assumption 2.2.2 still hold. We replace $\mu_{Y}, \sigma_{Y}$ and $b$ in all of the equations and definitions in Section 2.2 with $\widetilde{\mu}_{Y}, \widetilde{\sigma}_{Y}$ and $\widetilde{b}$, we get $\widetilde{H}^{*}$ and $\widetilde{H}_{*}$. Let $\widetilde{\mathbb{U}}^{+}$be the set of stochastic super-solutions of

$$
-\partial_{t} \varphi(t, x)+\widetilde{H}^{*} \varphi(t, x) \geq 0 \text { on } \mathbb{D}_{i}
$$

It is easy to see that $w \in \mathbb{U}^{+}$if and only if $\widetilde{w}(t, x):=e^{c t} w(t, x) \in \widetilde{\mathbb{U}}^{+}$. From Step 1 , $\widetilde{\mathbb{U}}^{+}$is not empty. Thus, $\mathbb{U}^{+}$is not empty.

Assumption 2.7.5. There is $C \in \mathbb{R}$ such that for all $(t, x, y, u, e) \in \mathbb{D} \times \mathbb{R} \times U \times E$,

$$
\left|\mu_{Y}(t, x, y, u)+\int_{E} b^{\top}(t, x, y, u(e), e) m(d e)\right| \leq C(1+|y|) .
$$

Proposition 2.7.6. Under Assumptions 2.2.1, 2.2.2, 2.7.1 and 2.7.5, $\mathbb{U}^{-}$is not empty.

Proof Assume that

$$
\mu_{Y}(t, x, y, u)+\int_{E} b^{\top}(t, x, y, u(e), e) m(d e)
$$


is non-decreasing in its $y$-variable. We could remove this assumption by using the argument from previous proposition. Choose $k \geq 2 C$ ( $C$ is the constant in Assumption 2.7.5) and $\gamma>0$ such that $e^{k T}-\gamma<-\|g\|_{\infty}$. Let $w(t, x)=e^{k x}-\gamma$. Notice that $w$ is continuous, has polynomial growth in $x$ and $w(T, \cdot) \leq g(\cdot)$. It suffices to show that for any $(t, x, y) \in \mathbb{D} \times \mathbb{R}, \tau \in \mathcal{T}_{t}$ and $\nu \in \mathcal{U}^{t}$, it holds that $\mathbb{P}(Y(\rho)<w(\rho, X(\rho)) \mid B)>0$ for all $\rho \in \mathcal{T}_{\tau}$ and $B \subset\{Y(\tau)<w(\tau, X(\tau))\}$ satisfying $B \in \mathcal{F}_{\tau}^{t}$ and $\mathbb{P}(B)>0$, where $X:=X_{t, x}^{\nu}$ and $Y:=Y_{t, x, y}^{\nu}$. Define

$$
\begin{gathered}
M(\cdot)=Y(\cdot)-\int_{\tau}^{\cdot} K(s) d s, \quad V(s)=w(s, X(s)), \\
A=\{Y(\tau)<w(\tau, X(\tau))\}, \quad \Gamma(s)=(Y(s)-V(s)) \mathbb{1}_{A},
\end{gathered}
$$

where

$$
\begin{aligned}
& K(s):=\mu_{Y}(s, X(s), Y(s), \nu(s))+\int_{E} b^{\top}\left(s, X(s-), Y(s-), \nu_{1}(s), \nu_{2}(s, e), e\right) m(d e) \\
& \widetilde{K}(s):=\mu_{Y}(s, X(s), V(s), \nu(s))+\int_{E} b^{\top}\left(s, X(s-), V(s-), \nu_{1}(s), \nu_{2}(s, e), e\right) m(d e) .
\end{aligned}
$$

It is easy to see that $M$ is a martingale after $\tau$. Due to the facts that $A \in \mathcal{F}_{\tau}^{t}$ and $d V(s)=k e^{k s} d s$, we further know

$$
\mathbb{1}_{A}\left(Y(\cdot)-V(\cdot)+\int_{\tau}^{\cdot} k e^{k s}-K(s) d s\right) \text { is a super-martingale after } \tau \text {. }
$$

Since Assumption 2.7.5 holds and $\mu_{Y}(t, x, y, u)+\int_{E} b^{\top}(t, x, y, u(e), e) m(d e)$ is nondecreasing in $y$,

$$
\widetilde{K}(s) \leq \mu_{Y}\left(s, X(s), e^{k s}, \nu(s)\right)+\int_{E} b^{\top}\left(s, X(s-), e^{k s}, \nu_{1}(s), \nu_{2}(s, e), e\right) m(d e) \leq 2 C e^{k s} .
$$

Therefore, it follows from (2.7.3) and the inequality above that

$$
\left.\widetilde{M}(\cdot):=\mathbb{1}_{A}\left(Y(\cdot)-V(\cdot)-\int_{\tau}^{\cdot} \xi(s) d s\right)\right) \text { is a super-martingale after } \tau,
$$

where $\xi(s):=K(s)-\widetilde{K}(s)$. Since $\widetilde{M}(\tau)<0$ on $B$, there exists a non-null set $F \subset B$ such that $\widetilde{M}(\rho)<0$ on $F$. By the definition of $\widetilde{M}$ in (2.7.4), we get

$$
\Gamma(\rho)<\mathbb{1}_{A} \int_{\tau}^{\rho} \xi(s) d s \text { on } F .
$$


Therefore,

$$
\Gamma^{+}(\rho) \leq \mathbb{1}_{A} \int_{\tau}^{\rho} \xi^{+}(s) d s \leq \int_{\tau}^{\rho} L_{0} \Gamma^{+}(s) d s \text { on } F
$$

By Grönwall's Inequality, $\Gamma^{+}(\tau)=0$ implies that $\Gamma^{+}(\rho)=0$ on $F$. More precisely, for $\omega \in F(\mathbb{P}-$ a.s. $), \Gamma^{+}(s)(\omega)=0$ for $s \in[\tau(\omega), \rho(\omega)]$. This implies that we can replace the inequalities with equalities in (2.7.6). Therefore, by $(2.7 .5), \Gamma(\rho)<0$ on $F$, which yields $\mathbb{P}(Y(\rho)<w(\rho, X(\rho)) \mid B)>0$. 


\section{CHAPTER III}

\section{Stochastic Perron for Stochastic Target Problems with a Stopper in a Jump Diffusion Model}

\subsection{Outline of this chapter}

In this chapter, we study stochastic target problems with a stopper in a jump diffusion model as in Chapter II. In Section 3.2, two types of the target problems (one with a cooperative stopper and the other with a non-cooperative stopper) and their associated HJB equations are introduced. In Sections 3.3 and 3.4, we analyze the stochastic target problem with a non-cooperative stopper and a cooperative stopper, resectively. More specifically, for the target problem with a non-cooperative stopper, we prove the viscosity properties in the parabolic interior and at the boundary, respectively in Subsections 3.3.1 and 3.3.2. In Subsection 3.3.3, we verify that the value function is the uniqueness viscosity solution to the associated HJB equation using comparison results. In Subsection 3.3.4, we see how a controller-stopper game can be converted into a target problem with a non-cooperative stopper. (A similar

outline applies to Section 3.4.) Some technical results are given in the appendix (Section 3.5). 


\subsection{The setup}

Given a complete probability space $(\Omega, \mathcal{F}, \mathbb{P})$, let $\left\{\lambda_{i}(\cdot, \text { de })\right\}_{i=1}^{I}$ be a collection of independent integer-valued $E$-marked right-continuous point processes defined on this space. Here, $E$ is a Borel subset of $\mathbb{R}$ equipped with the Borel sigma field $\mathcal{E}$. Let $\lambda=\left(\lambda_{1}, \lambda_{2}, \cdots, \lambda_{I}\right)^{\top}$ and $W=\left\{W_{s}\right\}_{0 \leq s \leq T}$ be a $d$-dimensional Brownian motion defined on the same probability space such that $W$ and $\lambda$ are independent. Given $t \in[0, T]$, let $\mathbb{F}^{t}=\left\{\mathcal{F}_{s}^{t}, t \leq s \leq T\right\}$ be $\mathbb{P}$-augmented filtration generated by $W .-W_{t}$ and $\lambda([0, \cdot], d e)-\lambda([0, t], d e)$. Set $\mathcal{F}_{s}^{t}=\mathcal{F}_{t}^{t}$ for $0 \leq s<t$. We will use $\mathcal{T}_{t}$ to denote the set of $\mathbb{F}^{t}$-stopping times valued in $[t, T]$. Given $\tau \in \mathcal{T}_{t}$, the set of $\mathbb{F}^{t}$-stopping times valued in $[\tau, T]$ will be denoted by $\mathcal{T}_{\tau}$.

Assumption 3.2.1. $\lambda$ satisfies the following:

1. $\lambda\left(d s\right.$, de) has intensity kernel $m(d e) d s$ such that $m_{i}$ is a Borel measure on $(E, \mathcal{E})$ for any $i=1, \cdots, I$ and $\hat{m}(E)<\infty$, where $m=\left(m_{1}, \cdots, m_{I}\right)^{\top}$ and $\hat{m}=$ $\sum_{i=1}^{I} m_{i}$

2. $E=\operatorname{supp}\left(m_{i}\right)$ for all $i=1,2, \cdots, I$. Here, $\operatorname{supp}\left(m_{i}\right):=\left\{e \in E: e \in N_{e} \in\right.$ $\left.T_{E} \Longrightarrow m_{i}\left(N_{e}\right)>0\right\}$, where $T_{E}$ is the topology on $E$ induced by the Euclidean topology.

3. There exists a constant $C>0$ such that

$$
\mathbb{P}(\{\hat{\lambda}(\{s\}, E) \leq C \text { for all } s \in[0, T]\})=1 \text {, where } \hat{\lambda}=\sum_{i=1}^{I} \lambda_{i} .
$$

The above assumption implies that there are a finite number of jumps during any finite time interval. Let $\tilde{\lambda}(d s, d e):=\lambda(d s, d e)-m(d e) d s$ be the associated compensated random measure.

Let $\mathcal{U}_{1}^{t}$ be the collection of all the $\mathbb{F}^{t}$-predictable processes in $\mathbb{L}^{2}(\Omega \times[0, T], \mathcal{F} \otimes$ $\left.\mathcal{B}[0, T], \mathbb{P} \otimes \lambda_{L} ; U_{1}\right)$, where $\lambda_{L}$ is the Lebesgue measure on $\mathbb{R}$ and $U_{1} \subset \mathbb{R}^{q}$ for some 
$q \in \mathbb{N}$. Define $\mathcal{U}_{2}^{t}$ to be the collection of all the maps $\nu_{2}: \Omega \times[0, T] \times E \rightarrow \mathbb{R}^{n}$ which are $\mathcal{P}^{t} \otimes \mathcal{E}$ measurable such that

$$
\left\|\nu_{2}\right\|_{\mathcal{U}_{2}^{t}}:=\left(\mathbb{E}\left[\int_{t}^{T} \int_{E}\left|\nu_{2}(s, e)\right|^{2} \hat{m}(d e) d s\right]\right)^{\frac{1}{2}}<\infty
$$

where $\mathcal{P}^{t}$ is the $\mathbb{F}^{t}$-predictable sigma-algebra on $\Omega \times[0, T] . \quad \nu=\left(\nu_{1}, \nu_{2}\right) \in \mathcal{U}_{0}^{t}:=$ $\mathcal{U}_{1}^{t} \times \mathcal{U}_{2}^{t}$ takes value in the set $U:=U_{1} \times \mathbb{L}^{2}\left(E, \mathcal{E}, \hat{m} ; \mathbb{R}^{n}\right)$. Let $\mathbb{D}=[0, T] \times \mathbb{R}^{d}$, $\mathbb{D}_{i}=[0, T) \times \mathbb{R}^{d}$ and $\mathbb{D}_{T}=\{T\} \times \mathbb{R}^{d}$. Given $z=(x, y) \in \mathbb{R}^{d} \times \mathbb{R}, t \in[0, T]$ and $\nu \in \mathcal{U}_{0}^{t}$, we consider the following SDEs.

$$
\begin{aligned}
d X(s) & =\mu_{X}(s, X(s), \nu(s)) d s+\sigma_{X}(s, X(s), \nu(s)) d W_{s} \\
& +\int_{E} \beta\left(s, X(s-), \nu_{1}(s), \nu_{2}(s, e), e\right) \lambda(d s, d e), \\
d Y(s) & =\mu_{Y}(s, Z(s), \nu(s)) d s+\sigma_{Y}^{\top}(s, Z(s), \nu(s)) d W_{s} \\
& +\int_{E} b^{\top}\left(s, Z(s-), \nu_{1}(s), \nu_{2}(s, e), e\right) \lambda(d s, d e),
\end{aligned}
$$

with $(X(t), Y(t))=(x, y)$. Here, $Z=(X, Y)$. In (3.2.1),

$$
\begin{gathered}
\mu_{X}: \mathbb{D} \times U \rightarrow \mathbb{R}^{d}, \quad \sigma_{X}: \mathbb{D} \times U \rightarrow \mathbb{R}^{d \times d}, \quad \beta: \mathbb{D} \times U_{1} \times \mathbb{R}^{n} \times E \rightarrow \mathbb{R}^{d \times I}, \\
\mu_{Y}: \mathbb{D} \times \mathbb{R} \times U \rightarrow \mathbb{R}, \quad \sigma_{Y}: \mathbb{D} \times \mathbb{R} \times U \rightarrow \mathbb{R}^{d}, \quad b: \mathbb{D} \times \mathbb{R} \times U_{1} \times \mathbb{R}^{n} \times E \rightarrow \mathbb{R}^{I} .
\end{gathered}
$$

Let $\mathcal{U}_{\text {unco }}^{t}$ be the admissible control set for the stochastic target problem with a non-cooperative stopper, which consists of all $\nu \in \mathcal{U}_{0}^{t}$ such that for any compact set $C \subset \mathbb{R}^{d} \times \mathbb{R}$ and $\tau \in \mathcal{T}_{t}$, there exists a constant $K_{\text {unco }}^{C, \nu, \tau}>0$ such that

$$
\int_{E} b^{\top}\left(\tau, x, y, \nu_{1}(\tau), \nu_{2}(\tau, e), e\right) \lambda(\{\tau\}, e) \geq-K_{\text {unco }}^{C, \nu, \tau} \text { for all }(x, y) \in C
$$

Let $\mathcal{U}_{\mathrm{co}}^{t}$ be the admissible control set for the stochastic target problem with a cooperative stopper, which consists of all $\nu \in \mathcal{U}_{0}^{t}$ such that for any compact set $C \subset \mathbb{R}^{d} \times \mathbb{R}$ and $\tau \in \mathcal{T}_{t}$, there exists a constant $K_{\text {co }}^{C, \nu, \tau}>0$ such that

$$
\int_{E} b^{\top}\left(\tau, x, y, \nu_{1}(\tau), \nu_{2}(\tau, e), e\right) \lambda(\{\tau\}, e) \leq K_{\mathrm{co}}^{C, \nu, \tau} \text { for all }(x, y) \in C
$$


Assumption 3.2.2. Let $z=(x, y)$ and $u=\left(u_{1}, u_{2}\right) \in U=U_{1} \times \mathbb{L}^{2}\left(E, \mathcal{E}, \hat{m} ; \mathbb{R}^{n}\right)$. We use the notation $\|u\|_{U}:=\left|u_{1}\right|+\left\|u_{2}\right\|_{\hat{m}}$ and $u(e):=\left(u_{1}, u_{2}(e)\right)$ for the rest of this chapter.

1. $\mu_{X}, \sigma_{X}, \mu_{Y}$ and $\sigma_{Y}$ are all continuous;

2. $\mu_{X}, \sigma_{X}, \mu_{Y}, \sigma_{Y}$ are Lipschitz in $z$ and locally Lipschitz in other variables. In addition,

$$
\begin{array}{r}
\left|\mu_{X}(t, x, u)\right|+\left|\sigma_{X}(t, x, u)\right| \leq L\left(1+|x|+\|u\|_{U}\right), \\
\left|\mu_{Y}(t, x, y, u)\right|+\left|\sigma_{Y}(t, x, y, u)\right| \leq L\left(1+|y|+\|u\|_{U}\right) .
\end{array}
$$

3. $b$ and $\beta$ are Lipschitz and grow linearly in all variables except e, but uniformly in e.

Remark 3.2.3. Assumptions 3.2.1 and 3.2.2 guarantee that there exists a unique strong solution $\left(X_{t, x}^{\nu}, Y_{t, x, y}^{\nu}\right)$ to (3.2.1) for any $\nu \in \mathcal{U}_{0}^{t}$. Moreover, the processes $\left(X_{t, x}^{\nu}\right.$, $\left.Y_{t, x, y}^{\nu}\right)$ are càdlàg.

We now define the value function of the stochastic target problems. Let $g: \mathbb{R}^{d} \rightarrow$ $\mathbb{R}$ be a continuous function with polynomial growth. The value functions of the target problems with a non-cooperative stopper and with a cooperative stopper are defined, respectively, by

$$
\begin{gathered}
u_{\text {unco }}(t, x):=\inf \left\{y: \exists \nu \in \mathcal{U}_{\text {unco }}^{t} \text { s.t. } Y_{t, x, y}^{\nu}(\rho) \geq g\left(X_{t, x}^{\nu}(\rho)\right) \text { for all } \rho \in \mathcal{T}_{t}\right\} \\
u_{\mathrm{co}}(t, x):=\sup \left\{y: \exists \nu \in \mathcal{U}_{\mathrm{co}}^{t} \text { and } \rho \in \mathcal{T}_{t} \text { s.t. } Y_{t, x, y}^{\nu}(\rho) \leq g\left(X_{t, x}^{\nu}(\rho)\right)\right\}
\end{gathered}
$$




\subsubsection{The Hamilton-Jacobi-Bellman equation}

Denote $b=\left(b_{1}, b_{2}, \cdots, b_{I}\right)^{\top}$ and $\beta=\left(\beta_{1}, \beta_{2}, \cdots, \beta_{I}\right)$. For a given $\varphi \in C(\mathbb{D})$, we define the relaxed semi-limits

$$
\begin{aligned}
& H^{*}(\Theta, \varphi):=\limsup _{\substack{\varepsilon \searrow 0, \Theta^{\prime} \rightarrow \Theta \\
\eta \searrow 0, \psi \stackrel{\text { u.c }}{\longrightarrow} \varphi}} H_{\varepsilon, \eta}\left(\Theta^{\prime}, \psi\right) \text { and } H_{*}(\Theta, \varphi):=\liminf _{\substack{\varepsilon \searrow 0, \Theta^{\prime} \rightarrow \Theta \\
\eta \searrow 0, \psi \stackrel{\text { u.c. }}{\longrightarrow} \varphi}} H_{\varepsilon, \eta}\left(\Theta^{\prime}, \psi\right),{ }^{1} \\
& F^{*}(\Theta, \varphi):=\limsup _{\substack{\varepsilon \searrow 0, \Theta^{\prime} \rightarrow \Theta \\
\eta \searrow 0, \psi \stackrel{\text { u.c. }}{\rightarrow} \varphi}} F_{\varepsilon, \eta}\left(\Theta^{\prime}, \psi\right) \text { and } F_{*}(\Theta, \varphi):=\liminf _{\substack{\varepsilon \searrow 0, \Theta^{\prime} \rightarrow \Theta \\
\eta \searrow 0, \psi \stackrel{\text { u.c. }}{\longrightarrow} \varphi}} F_{\varepsilon, \eta}\left(\Theta^{\prime}, \psi\right)
\end{aligned}
$$

Here, for $\Theta=(t, x, y, p, A) \in \mathbb{D} \times \mathbb{R} \times \mathbb{R}^{d} \times \mathbb{M}^{d}, \varphi \in C(\mathbb{D}), \varepsilon \geq 0$ and $\eta \in[-1,1]$,

$$
\begin{gathered}
H_{\varepsilon, \eta}(\Theta, \varphi):=\sup _{u \in \mathcal{N}_{\varepsilon, \eta}(t, x, y, p, \varphi)} \mathbf{L}^{u}(\Theta), F_{\varepsilon, \eta}(\Theta, \varphi):=\inf _{u \in \mathcal{M}_{\varepsilon, \eta}(t, x, y, p, \varphi)} \mathbf{L}^{u}(\Theta), \text { where, } \\
\mathbf{L}^{u}(\Theta):=\mu_{Y}(t, x, y, u)-\mu_{X}^{\top}(t, x, u) p-\frac{1}{2} \operatorname{Tr}\left[\sigma_{X} \sigma_{X}^{\top}(t, x, u) A\right], \\
N^{u}(t, x, y, p):=\sigma_{Y}(t, x, y, u)-\sigma_{X}^{\top}(t, x, u) p \\
\Delta^{u, e}(t, x, y, \varphi):=\min _{1 \leq i \leq I}\left\{b_{i}(t, x, y, u(e), e)-\varphi\left(t, x+\beta_{i}(t, x, u(e), e)\right)+\varphi(t, x)\right\}, \\
\Pi^{u, e}(t, x, y, \varphi):=\max _{1 \leq i \leq I}\left\{b_{i}(t, x, y, u(e), e)-\varphi\left(t, x+\beta_{i}(t, x, u(e), e)\right)+\varphi(t, x)\right\}, \\
\mathcal{N}_{\varepsilon, \eta}(t, x, y, p, \varphi):=\left\{u \in U:\left|N^{u}(t, x, y, p)\right| \leq \varepsilon \text { and } \Delta^{u, e}(t, x, y, \varphi) \geq \eta \hat{m} \text {-a.s. }\right\}, \\
\mathcal{M}_{\varepsilon, \eta}(t, x, y, p, \varphi):=\left\{u \in U:\left|N^{u}(t, x, y, p)\right| \leq \varepsilon \text { and } \Pi^{u, e}(t, x, y, \varphi) \leq \eta \hat{m} \text {-a.s. }\right\} .
\end{gathered}
$$

For our later use, we also define the following:

$$
\begin{gathered}
J_{i}^{u, e}(t, x, y, \varphi):=b_{i}(t, x, y, u(e), e)-\varphi\left(t, x+\beta_{i}(t, x, u(e), e)\right)+\varphi(t, x), \\
\bar{J}^{u, e}(t, x, y, \varphi):=\left(J_{1}^{u, e}(t, x, y, \varphi), \cdots, J_{I}^{u, e}(t, x, y, \varphi)\right)^{\top} \\
\mathscr{L}^{u} \varphi(t, x):=\varphi_{t}(t, x)+\mu_{X}^{\top}(t, x, u) D \varphi(t, x)+\frac{1}{2} \operatorname{Tr}\left[\sigma_{X} \sigma_{X}^{\top}(t, x, u) D^{2} \varphi(t, x)\right] .
\end{gathered}
$$

Definition 3.2.4 (Concatenation). Let $\nu_{1}, \nu_{2} \in \mathcal{U}_{\text {unco }}^{t}\left(\operatorname{resp} . \mathcal{U}_{\text {co }}^{t}\right), \tau \in \mathcal{T}_{t}$. The concatenation of $\nu_{1}$ and $\nu_{2}$ at $\tau$ is defined as $\nu_{1} \otimes_{\tau} \nu_{2}:=\nu_{1} \mathbb{1}_{[0, \tau[}+\nu_{2} \mathbb{1}_{[\tau, T]} \in \mathcal{U}_{\text {unco }}^{t}$ $\left(\operatorname{resp} . \mathcal{U}_{\mathrm{co}}^{t}\right) .^{2}$

${ }^{1}$ The convergence $\psi \stackrel{\text { u.c. }}{\longrightarrow} \varphi$ is understood in the sense that $\psi$ converges uniformly on compact subsets to $\varphi$.

${ }^{2}$ This can be easily checked. 


\subsection{The stochastic target problem with a non-cooperative stopper}

Definition 3.3.1 (Stochastic super-solutions). A continuous function $w: \mathbb{D} \rightarrow \mathbb{R}$ is called a stochastic super-solution if

1. $w(t, x) \geq g(x)$ and for some $C>0$ and $n \in \mathbb{N},{ }^{3}|w(t, x)| \leq C\left(1+|x|^{n}\right)$ for all $(t, x) \in \mathbb{D}$.

2. Given $(t, x, y) \in \mathbb{D} \times \mathbb{R}$, for any $\tau \in \mathcal{T}_{t}$ and $\nu \in \mathcal{U}_{\text {unco }}^{t}$, there exists $\tilde{\nu} \in \mathcal{U}_{\text {unco }}^{t}$ such that

$$
Y(\rho) \geq w(\rho, X(\rho)) \quad \mathbb{P} \text {-a.s. on }\{Y(\tau) \geq w(\tau, X(\tau))\}
$$

for all $\rho \in \mathcal{T}_{\tau}$, where $X:=X_{t, x}^{\nu \otimes_{\tau} \tilde{\nu}}$ and $Y:=Y_{t, x, y}^{\nu \otimes_{\tau} \tilde{\nu}}$.

Denote the sets of stochastic super-solutions by $\mathbb{U}_{\text {unco }}^{+}$.

Definition 3.3.2 (Stochastic sub-solutions). A continuous function $w: \mathbb{D} \rightarrow \mathbb{R}$ is called a stochastic sub-solution if

1. $w(T, x) \leq g(x)$ and for some $C>0$ and $n \in \mathbb{N},|w(t, x)| \leq C\left(1+|x|^{n}\right)$ for all $(t, x) \in \mathbb{D}$.

2. Given $(t, x, y) \in \mathbb{D} \times \mathbb{R}$, for any $\tau \in \mathcal{T}_{t}, \nu \in \mathcal{U}_{\text {unco }}^{t}$ and $B \subset\{Y(\tau)<w(\tau, X(\tau))\}$ satisfying $B \in \mathcal{F}_{\tau}^{t}$ and $\mathbb{P}(B)>0$, there exists $\rho \in \mathcal{T}_{\tau}$ such that

$$
\mathbb{P}(Y(\rho)<g(X(\rho)) \mid B)>0
$$

Here, we use the notation $X:=X_{t, x}^{\nu}$ and $Y:=Y_{t, x, y}^{\nu}$.

Denote the sets of stochastic sub-solutions by $\mathbb{U}_{\text {unco }}^{-}$.

Assumption 3.3.3. $\mathbb{U}_{\text {unco }}^{+}$and $\mathbb{U}_{\text {unco }}^{-}$are not empty.

\footnotetext{
${ }^{3} C$ and $N$ may depend on $w$ and $T$. This also applies to Definition 3.3.2, 3.4.1 and 3.4.2.
} 
We will provide sufficient conditions which guarantee Assumption 3.3.3 in Subsection 3.5.1. When the assumption above holds, by similar arguments as in Remark 2.2.9 and 2.2.10, we get that

$$
u_{\text {unco }}^{-}:=\sup _{w \in \mathbb{U}_{\text {unco }}^{-}} w \leq u_{\text {unco }} \leq \inf _{w \in \mathbb{U}_{\text {unco }}^{+}} w=: u_{\text {unco }}^{+}
$$

\subsubsection{Viscosity property in $\mathbb{D}_{i}$}

In this subsection, we state and prove the theorem which characterizes $u_{\text {unco }}^{-}$as a viscosity super-solution of

$$
\min \left\{\varphi(t, x)-g(x),-\partial_{t} \varphi(t, x)+H^{*} \varphi(t, x)\right\} \geq 0 \text { in } \mathbb{D}_{i}
$$

and $u_{\text {unco }}^{+}$as a viscosity sub-solution of

$$
\min \left\{\varphi(t, x)-g(x),-\partial_{t} \varphi(t, x)+H_{*} \varphi(t, x)\right\} \leq 0 \text { in } \mathbb{D}_{i}
$$

The boundary conditions will be discussed in Theorem 3.3.8. The same assumption as Assumption 2.2.11 is needed for the sub-solution property of $u_{\text {unco }}^{-}$.

Assumption 3.3.4. For $\psi \in C(\mathbb{D}), \eta>0$, let $B$ be a subset of $\mathbb{D} \times \mathbb{R} \times \mathbb{R}^{d}$ such that $\mathcal{N}_{0, \eta}(\cdot, \psi) \neq \emptyset$ on $B$. Then for every $\varepsilon>0,\left(t_{0}, x_{0}, y_{0}, p_{0}\right) \in \operatorname{Int}(B)$ and $u_{0} \in$ $\mathcal{N}_{0, \eta}\left(t_{0}, x_{0}, y_{0}, p_{0}, \psi\right)$, there exists an open neighborhood $B^{\prime}$ of $\left(t_{0}, x_{0}, y_{0}, p_{0}\right)$ and a locally Lipschitz continuous map $\hat{\nu}$ defined on $B^{\prime}$ such that $\left\|\hat{\nu}\left(t_{0}, x_{0}, y_{0}, p_{0}\right)-u_{0}\right\|_{U} \leq \varepsilon$ and $\hat{\nu}(t, x, y, p) \in \mathcal{N}_{0, \eta}(t, x, y, p, \psi)$.

Lemma 3.3.5. $\mathbb{U}_{\text {unco }}^{+}$and $\mathbb{U}_{\text {unco }}^{-}$are closed under pairwise minimization and maximization, respectively.

Lemma 3.3.6. There exists a non-increasing sequence $\left\{w_{n}\right\}_{n=1}^{\infty} \subset \mathbb{U}_{\text {unco }}^{+}$such that $w_{n} \searrow u_{\text {unco }}^{+}$and a non-decreasing sequence $\left\{v_{n}\right\}_{n=1}^{\infty} \subset \mathbb{U}_{\text {unco }}^{-}$such that $v_{n} \nearrow u_{\text {unco }}^{-}$. 
Theorem 3.3.7. Under Assumptions 3.2.1, 3.2.2, 3.3.3 and 3.3.4, $u_{\text {unco }}^{+}$is a USC viscosity sub-solution of (3.3.3). On the other hand, under Assumptions 3.2.1, 3.2.2 and 3.3.3, $u_{\text {unco }}^{-}$is an LSC viscosity super-solution of (3.3.2).

Proof. Step 1 ( $u_{\text {unco }}^{+}$is a viscosity sub-solution). Assume, on the contrary, that for some $\left(t_{0}, x_{0}\right) \in \mathbb{D}_{i}$ and $\varphi \in C^{1,2}(\mathbb{D})$ satisfying $0=\left(u_{\text {unco }}^{+}-\varphi\right)\left(t_{0}, x_{0}\right)=$ $\max _{\mathbb{D}_{i}}\left(u_{\text {unco }}^{+}-\varphi\right)$, we have

$$
4 \eta:=-\partial_{t} \varphi\left(t_{0}, x_{0}\right)+H_{*} \varphi\left(t_{0}, x_{0}\right)>0 \quad \text { and } \quad \varphi\left(t_{0}, x_{0}\right)>g\left(x_{0}\right)
$$

From Lemma 3.3.6, there exists a non-increasing sequence $\mathbb{U}_{\text {unco }}^{+} \ni w_{k} \searrow u_{\text {unco }}^{+}$. Fix such a sequence $\left\{w_{k}\right\}_{k=1}^{\infty}$ and an arbitrary stochastic sub-solution $w_{-}$. Let $\tilde{\varphi}(t, x)=$ $\varphi(t, x)+\iota\left|x-x_{0}\right|^{n_{0}} \cdot{ }^{4}$ We can choose $n_{0} \geq 2$ such that for any $\iota>0$,

$$
\min _{0 \leq t \leq T}\left(\tilde{\varphi}(t, x)-w_{1}(t, x)\right) \rightarrow \infty \text { as }|x| \rightarrow \infty
$$

We can do this because $\varphi$ is bounded from below by $w_{-}$(which has polynomial growth in $x)$ and $w_{1}$ has polynomial growth in $x$. Since $\left(\mathcal{N}_{\varepsilon, \eta}\right)_{\varepsilon \geq 0}$ is non-decreasing in $\varepsilon$, we know

$$
H_{*}(\Theta, \varphi)=\liminf _{\substack{\Theta^{\prime} \rightarrow \Theta, \psi \stackrel{\text { u.c. }}{\eta} \eta \\ \eta \searrow 0}} H_{0, \eta}\left(\Theta^{\prime}, \psi\right) .
$$

By (3.2.6) and (3.3.4), we can find $\varepsilon>0, \eta>0$ and $\iota>0$ such that for all $(t, x, y)$ satisfying $(t, x) \in B_{\varepsilon}\left(t_{0}, x_{0}\right)$ and $|y-\tilde{\varphi}(t, x)| \leq \varepsilon, \mu_{Y}(t, x, y, u)-\mathcal{L}^{u} \tilde{\varphi}(t, x) \geq$ $2 \eta$ for some $u \in \mathcal{N}_{0, \eta}(t, x, y, D \tilde{\varphi}(t, x), \tilde{\varphi})$ and $\tilde{\varphi}(t, x)>g(x)+\varepsilon$. Fix $\iota$. Note that $\left(t_{0}, x_{0}\right)$ is still a strict maximizer of $u_{\text {unco }}^{+}-\tilde{\varphi}$ over $\mathbb{D}_{i}$. For $\varepsilon$ sufficiently small,

\footnotetext{
${ }^{4}$ Since we will fix $n_{0}$ and $\iota$ later, we still use the notation $\tilde{\varphi}$ when without ambiguity despite the fact that the function depends on $n_{0}$ and $\iota$.
} 
Assumption 3.3.4 implies that there exists a locally Lipschitz map $\hat{\nu}$ such that

$$
\begin{gathered}
\qquad \hat{\nu}(t, x, y, D \tilde{\varphi}(t, x)) \in \mathcal{N}_{0, \eta}(t, x, y, D \tilde{\varphi}(t, x), \tilde{\varphi}) \text { and } \\
\qquad \mu_{Y}(t, x, y, \hat{\nu}(t, x, y, D \tilde{\varphi}(t, x)))-\mathcal{L}^{\hat{\nu}(t, x, y, D \tilde{\varphi}(t, x))} \tilde{\varphi}(t, x) \geq \eta \\
\text { for all }(t, x, y) \in \mathbb{D}_{i} \times \mathbb{R} \text { s.t. }(t, x) \in B_{\varepsilon}\left(t_{0}, x_{0}\right) \text { and }|y-\tilde{\varphi}(t, x)| \leq \varepsilon .
\end{gathered}
$$

In the arguments above, choose $\varepsilon$ small enough such that $\bar{B}_{\varepsilon}\left(t_{0}, x_{0}\right) \cap \mathbb{D}_{T}=\emptyset$. Since (3.3.5) holds, there exists $R_{0}>\varepsilon$ such that $\tilde{\varphi}>w_{1}+\varepsilon \geq w_{k}+\varepsilon$ on $\mathbb{O}:=$ $\mathbb{D}-[0, T] \times \bar{B}_{R_{0}}\left(x_{0}\right)$ for all $k$. On the compact set $\mathbb{T}:=[0, T] \times \bar{B}_{R_{0}}\left(x_{0}\right)-B_{\varepsilon / 2}\left(t_{0}, x_{0}\right)$, we know that $\tilde{\varphi}>u_{\text {unco }}^{+}$and the minimum of $\tilde{\varphi}-u_{\text {unco }}^{+}$is attained since $u_{\text {unco }}^{+}$is USC. Therefore, $\tilde{\varphi}>u_{\text {unco }}^{+}+2 \alpha$ on $\mathbb{T}$ for some $\alpha>0$. By a Dini-type argument, for large enough $n$, we have $\tilde{\varphi}>w_{n}+\alpha$ on $\mathbb{T}$ and $\tilde{\varphi}>w_{n}-\varepsilon$ on $\bar{B}_{\varepsilon / 2}\left(t_{0}, x_{0}\right)$. For simplicity, fix such an $n$ and set $w=w_{n}$. In short,

$$
\tilde{\varphi}>w+\varepsilon \text { on } \mathbb{O}, \tilde{\varphi}>w+\alpha \text { on } \mathbb{T} \text { and } \tilde{\varphi}>w-\varepsilon \text { on } \bar{B}_{\varepsilon / 2}\left(t_{0}, x_{0}\right)
$$

For $\kappa \in(0, \varepsilon \wedge \alpha)$, define

$$
w^{\kappa}:=\left\{\begin{array}{l}
(\tilde{\varphi}-\kappa) \wedge w \text { on } \bar{B}_{\varepsilon}\left(t_{0}, x_{0}\right), \\
w \text { outside } \bar{B}_{\varepsilon}\left(t_{0}, x_{0}\right) .
\end{array}\right.
$$

Observing that $w^{\kappa}\left(t_{0}, x_{0}\right)=\tilde{\varphi}\left(t_{0}, x_{0}\right)-\kappa<u_{\text {unco }}^{+}\left(t_{0}, x_{0}\right)$, we could obtain a contradiction if we could show that $w^{\kappa} \in \mathbb{U}_{\text {unco }}^{+}$. Obviously, $w^{\kappa}$ is continuous, has polynomial growth in $x$ and $w^{\kappa}(t, x) \geq g(x)$ for all $(t, x) \in \mathbb{D}$. Fix $(t, x, y) \in \mathbb{D}_{i} \times \mathbb{R}, \nu \in \mathcal{U}_{\text {unco }}^{t}$ and $\tau \in \mathcal{T}_{t}{ }^{5}$ It suffices to construct a $\widetilde{\nu} \in \mathbb{U}_{\text {unco }}^{t}$ such that $w^{\kappa}$ and the processes $(X, Y)$ controlled by $\nu \otimes_{\tau} \widetilde{\nu}$ satisfy the property in Definition 3.3.1. The construction of such $\widetilde{\nu}$ follows from the same arguments in Step 1 of Theorem 2.3.3's proof.

\section{Step $2\left(u_{\text {unco }}^{-}\right.$is a viscosity super-solution).}

Step A: We show in this step that $u_{\text {unco }}^{-}(t, x) \geq g(x)$ for all $(t, x) \in \mathbb{D}$. Assume, on

\footnotetext{
${ }^{5}$ Here we choose $(t, x) \in \mathbb{D}_{i}$ since the case $(t, x) \in \mathbb{D}_{T}$ is trivial.
} 
the contrary, that for some $\left(t_{0}, x_{0}\right) \in \mathbb{D}$, there exists $\eta>0$ such that

$$
2 \eta=g\left(x_{0}\right)-u_{\text {unco }}^{-}\left(t_{0}, x_{0}\right)>0 .
$$

Choose an arbitrary $w \in \mathbb{U}_{\text {unco }}^{-}$. By the definition of $\mathbb{U}_{\text {unco }}^{-}$and lower semi-continuity of $g$, there exists $\varepsilon>0$ such that for all $(t, x) \in \bar{B}_{\varepsilon}\left(t_{0}, x_{0}\right)$.

$$
g(x)-w(t, x)>\eta, g(x)-g\left(x_{0}\right)>-\frac{\eta}{2},\left|w(t, x)-w\left(t_{0}, x_{0}\right)\right| \leq \frac{\eta}{2} .
$$

Define

$$
w^{\prime}(t, x):=\left\{\begin{array}{l}
w(t, x), \text { for }(t, x) \notin \bar{B}_{\varepsilon}\left(t_{0}, x_{0}\right), \\
w(t, x)+\left(g\left(x_{0}\right)-\eta-w\left(t_{0}, x_{0}\right)\right)\left(1-\frac{\operatorname{dist}\left((t, x),\left(t_{0}, x_{0}\right)\right)}{\varepsilon}\right), \text { otherwise. }
\end{array}\right.
$$

Obviously, $w^{\prime} \geq w$ and $w^{\prime}$ is continuous with polynomial growth. In addition,

$$
w^{\prime}(t, x) \leq w(t, x)+\left(g\left(x_{0}\right)-\eta-w\left(t_{0}, x_{0}\right)\right)<g(x) \text { for }(t, x) \in \bar{B}_{\varepsilon}\left(t_{0}, x_{0}\right) .
$$

The equation above, along with the fact that $w \in \mathbb{U}_{\text {unco }}^{-}$, implies that $w^{\prime}(T, x) \leq g(x)$ for all $x \in \mathbb{R}^{d}$. Noting that $w^{\prime}\left(t_{0}, x_{0}\right)=g\left(x_{0}\right)-\eta>u_{\text {unco }}^{-}\left(t_{0}, x_{0}\right)$ due to (3.3.9), we would obtain a contradiction if we could show $w^{\prime} \in \mathbb{U}_{\text {unco }}^{-}$.

To prove that $w^{\prime} \in \mathbb{U}_{\text {unco }}^{-}$, fix $(t, x, y) \in \mathbb{D}_{i} \times \mathbb{R}, \tau \in \mathcal{T}_{t}$ and $\nu \in \mathcal{U}_{\text {unco }}^{t}$. For $w \in \mathbb{U}_{\text {unco }}^{-}$, let $\rho^{w, \tau, \nu} \in \mathcal{T}_{\tau}$ be the "optimal" stopping time satisfying the second item in Definition 3.3.2. In order to show that $w^{\prime} \in \mathbb{U}_{\text {unco }}^{-}$, we want to construct an "optimal" stopping time $\rho$ which works in the sense of Definition 3.3.2. Let $A=\left\{w(\tau, X(\tau))=w^{\prime}(\tau, X(\tau))\right\} \in \mathcal{F}_{\tau}^{t}$ and

$$
\rho=\mathbb{1}_{A} \rho^{w, \tau, \nu}+\mathbb{1}_{A^{c}} \tau
$$


Obviously, $\rho \in \mathcal{T}_{\tau}$. It suffices to show $\mathbb{P}(Y(\rho)<g(X(\rho)) \mid B)>0$ for any $B \subset$ $\left\{Y(\tau)<w^{\prime}(\tau, X(\tau))\right\}$ satisfying $\mathbb{P}(B)>0$ and $B \in \mathcal{F}_{\tau}^{t}$. The following two scenarios together will yield the desired result.

(i) If $\mathbb{P}(B \cap A)>0$ : We know that $B \cap A \subset\{Y(\tau)<w(\tau, X(\tau))\}$ and $B \cap A \in \mathcal{F}_{\tau}^{t}$. From the fact $w \in \mathbb{U}_{\text {unco }}^{-}$and the definition of $\rho$ on $A$, it holds that

$$
\mathbb{P}(Y(\rho)<g(X(\rho)) \mid B \cap A)=\mathbb{P}\left(Y\left(\rho^{w, \tau, \nu}\right)<g\left(X\left(\rho^{w, \tau, \nu}\right)\right) \mid B \cap A\right)>0 .
$$

(ii) If $\mathbb{P}\left(B \cap A^{c}\right)>0:(\tau, X(\tau)) \in B_{\varepsilon}\left(t_{0}, x_{0}\right)$ on $A^{c}$ from (3.3.10), which implies $w^{\prime}(\tau, X(\tau))<g(X(\tau))$ from (3.3.11). Since $\rho=\tau$ on $A^{c}$

$$
\mathbb{P}\left(Y(\rho)<g(X(\rho)) \mid B \cap A^{c}\right) \geq \mathbb{P}\left(Y(\tau)<w^{\prime}(\tau, X(\tau)) \mid B \cap A^{c}\right)=\mathbb{P}\left(B \cap A^{c}\right)>0
$$

Step B: Let $\left(t_{0}, x_{0}\right) \in \mathbb{D}_{i}$ satisfy $0=\left(u_{\text {unco }}^{-}-\varphi\right)\left(t_{0}, x_{0}\right)=\min _{\mathbb{D}_{i}}\left(u_{\text {unco }}^{-}-\varphi\right)$ for some $\varphi \in C^{1,2}(\mathbb{D})$. For the sake of contradiction, assume that

$$
-2 \eta:=-\partial_{t} \varphi\left(t_{0}, x_{0}\right)+H^{*} \varphi\left(t_{0}, x_{0}\right)<0
$$

Let $\left\{w_{k}\right\}_{k=1}^{\infty}$ be a sequence in $\mathbb{U}_{\text {unco }}^{-}$such that $w_{k} \nearrow u_{\text {unco }}^{-}$. Let $\tilde{\varphi}(t, x):=\varphi(t, x)-$ $\iota\left|x-x_{0}\right|^{n_{0}}$, where we choose $n_{0} \geq 2$ such that for all $\iota>0$,

$$
\max _{0 \leq t \leq T}\left(\tilde{\varphi}(t, x)-w_{1}(t, x)\right) \rightarrow-\infty \text { and } \max _{0 \leq t \leq T} \tilde{\varphi}(t, x) \rightarrow-\infty \text { as }|x| \rightarrow \infty .^{6}
$$

By (3.3.12), the upper semi-continuity of $H^{*}$ and the fact that $\tilde{\varphi} \stackrel{\text { u.c. }}{\longrightarrow} \varphi$ as $\iota \rightarrow 0$, we can find $\varepsilon>0, \eta>0$ and $\iota>0$ such that

$$
\begin{gathered}
\mu_{Y}(t, x, y, u)-\mathscr{L}^{u} \tilde{\varphi}(t, x) \leq-\eta \text { for all } u \in \mathcal{N}_{\varepsilon,-\eta}(t, x, y, D \tilde{\varphi}(t, x), \tilde{\varphi}) \\
\text { and }(t, x, y) \in \mathbb{D}_{i} \times \mathbb{R} \text { s.t. }(t, x) \in B_{\varepsilon}\left(t_{0}, x_{0}\right) \text { and }|y-\tilde{\varphi}(t, x)| \leq \varepsilon .
\end{gathered}
$$

Fix $\iota$. Note that $\left(t_{0}, x_{0}\right)$ is still a strict minimizer of $u_{\text {unco }}^{-}-\tilde{\varphi}$. Since (3.3.13) holds, there exists $R_{0}>\varepsilon$ such that

$$
\tilde{\varphi}<w_{1}-\varepsilon \leq w_{k}-\varepsilon \text { on } \mathbb{O}:=\mathbb{D}-[0, T] \times \bar{B}_{R_{0}}\left(x_{0}\right) .
$$

\footnotetext{
${ }^{6}$ The existence of $n_{0}$ follows as in Step1.
} 
On the compact set $\mathbb{T}:=[0, T] \times \bar{B}_{R_{0}}\left(x_{0}\right)-B_{\varepsilon / 2}\left(t_{0}, x_{0}\right)$, we know that $\tilde{\varphi}<u_{\text {unco }}^{-}$and the maximum of $\tilde{\varphi}-u_{\text {unco }}^{-}$is attained since $u_{\text {unco }}^{-}$is LSC. Therefore, $\tilde{\varphi}<u_{\text {unco }}^{-}-2 \alpha$ on $\mathbb{T}$ for some $\alpha>0$. By a Dini-type argument, for large enough $n$, we have $\tilde{\varphi}<w_{n}-\alpha$ on $\mathbb{T}$ and $\tilde{\varphi}<w_{n}+\varepsilon$ on $\bar{B}_{\varepsilon / 2}\left(t_{0}, x_{0}\right)$. For simplicity, fix such an $n$ and set $w=w_{n}$. In short,

$$
\tilde{\varphi}<w-\varepsilon \text { on } \mathbb{O}, \tilde{\varphi}<w-\alpha \text { on } \mathbb{T} \text { and } \tilde{\varphi}<w+\varepsilon \text { on } \bar{B}_{\varepsilon / 2}\left(t_{0}, x_{0}\right) \text {. }
$$

For $\kappa \in(0, \alpha \wedge \varepsilon)$, define

$$
w^{\kappa}:=\left\{\begin{array}{l}
(\tilde{\varphi}+\kappa) \vee w \text { on } \bar{B}_{\varepsilon}\left(t_{0}, x_{0}\right), \\
w \text { outside } \bar{B}_{\varepsilon}\left(t_{0}, x_{0}\right) .
\end{array}\right.
$$

Noticing that $w^{\kappa}\left(t_{0}, x_{0}\right) \geq \tilde{\varphi}\left(t_{0}, x_{0}\right)+\kappa>u_{\text {unco }}^{-}\left(t_{0}, x_{0}\right)$, we will obtain a contradiction if we show that $w^{\kappa} \in \mathbb{U}_{\text {unco }}^{-}$. Obviously, $w^{\kappa}$ is continuous, has polynomial growth in $x$ and $w^{\kappa}(T, x) \leq g(x)$ for all $x \in \mathbb{R}^{d}$. Fix $(t, x, y) \in \mathbb{D}_{i} \times \mathbb{R}, \nu \in \mathcal{U}_{\text {unco }}^{t}$ and $\tau \in \mathcal{T}_{t}$. Let $X=X_{t, x}^{\nu}, Y=Y_{t, x, y}^{\nu}$ and $\theta=\theta_{1} \wedge \theta_{2}$, where

$$
\begin{aligned}
& \theta_{1}:=\inf \left\{s \in[\tau, T]:(s, X(s)) \notin B_{\varepsilon / 2}\left(t_{0}, x_{0}\right)\right\} \wedge T, \\
& \theta_{2}:=\inf \{s \in[\tau, T]:|Y(s)-\tilde{\varphi}(s, X(s))| \geq \varepsilon\} \wedge T .
\end{aligned}
$$

Since $X$ and $Y$ are càdlàg processes, we know that $\theta \in \mathcal{T}_{\tau}$ and the following hold:

$$
\begin{gathered}
\left(\theta_{1}, X\left(\theta_{1}\right)\right) \notin B_{\varepsilon / 2}\left(t_{0}, x_{0}\right), \quad\left|Y\left(\theta_{2}\right)-\tilde{\varphi}\left(\theta_{2}, X\left(\theta_{2}\right)\right)\right| \geq \varepsilon, \\
\left.\left(\theta_{1}, X\left(\theta_{1}-\right)\right) \in \bar{B}_{\varepsilon / 2}\left(t_{0}, x_{0}\right)\right), \quad\left|Y\left(\theta_{2}-\right)-\tilde{\varphi}\left(\theta_{2}, X\left(\theta_{2}-\right)\right)\right| \leq \varepsilon .
\end{gathered}
$$

Let $A=\left\{w^{\kappa}(\tau, X(\tau))=w(\tau, X(\tau))\right\}$ and

$$
\rho=\mathbb{1}_{A} \rho^{w, \tau, \nu}+\mathbb{1}_{A^{c}} \rho^{w, \theta, \nu}
$$

where $\rho^{w, \tau, \nu}$ (resp. $\rho^{w, \theta, \nu}$ ) is the "optimal" stopping time in Definition 3.3.2 for $w$ given $\tau($ resp. $\theta)$ and $\nu$. To prove that $w^{\kappa} \in \mathbb{U}_{\text {unco }}^{-}$, it suffices to show that

$$
\mathbb{P}(Y(\rho)<g(X(\rho)) \mid B)>0
$$


for $B \subset\left\{Y(\tau)<w^{\kappa}(\tau, X(\tau))\right\}$ satisfying $B \in \mathcal{F}_{\tau}^{t}$ and $\mathbb{P}(B)>0$. Let

$$
E=\left\{Y(\tau)<w^{\kappa}(\tau, X(\tau))\right\}, \quad E_{0}=E \cap A, \quad E_{1}=E \cap A^{c}, \quad G=\{Y(\rho)<g(X(\rho)\}
$$

Then $E=E_{0} \cup E_{1}$ and $E_{0} \cap E_{1}=\emptyset$. Therefore, we want to show that $\mathbb{P}(G \cap B)>0$.

We will show

$$
\begin{aligned}
& \mathbb{P}\left(B \cap E_{0}\right)>0 \Longrightarrow \mathbb{P}\left(G \cap B \cap E_{0}\right)>0 \text { and } \\
& \mathbb{P}\left(B \cap E_{1}\right)>0 \Longrightarrow \mathbb{P}\left(G \cap B \cap E_{1}\right)>0 .
\end{aligned}
$$

This, together with the facts $\mathbb{P}(B)=\mathbb{P}\left(B \cap E_{0}\right)+\mathbb{P}\left(B \cap E_{1}\right)>0$ and $\mathbb{P}(G \cap B)=$ $\mathbb{P}\left(G \cap B \cap E_{0}\right)+\mathbb{P}\left(G \cap B \cap E_{1}\right)$, implies that $\mathbb{P}(G \cap B)>0$.

(i)Assume that $\mathbb{P}\left(B \cap E_{0}\right)>0$. Since $B \cap E_{0} \subset\{Y(\tau)<w(\tau, X(\tau))\}$ and $B \cap E_{0} \in \mathcal{F}_{\tau}^{t}, \mathbb{P}\left(G \mid B \cap E_{0}\right)>0$ from the fact $w \in \mathbb{U}_{\text {unco }}^{-}$and the definition of $\rho$ on $A$. This further implies that $\mathbb{P}\left(G \cap B \cap E_{0}\right)>0$.

(ii) Assume that $\mathbb{P}\left(B \cap E_{1}\right)>0$. Let $\Gamma(s):=Y(s)-\widetilde{\varphi}(s, X(s))-\kappa$. From the arguments in Step 2 of Theorem 2.3.3's proof, we know that $\Gamma L$ is a super-martingale, where $L(\cdot)$ is a positive local martingale. Since $\Gamma(\tau) L(\tau)<0$ on $B \cap E_{1}$, the supermartingale property of $\Gamma L$ implies that there exists $F \subset B \cap E_{1}$ such that $F \in \mathcal{F}_{\tau}^{t}$ and $\Gamma(\theta) L(\theta)<0$ on $F$. The non-negativity of $L$ then yields $\Gamma(\theta)<0$ on $F$. Therefore,

$$
\begin{aligned}
& Y\left(\theta_{1}\right)<\tilde{\varphi}\left(\theta_{1}, X\left(\theta_{1}\right)\right)+\kappa \text { on } F \cap\left\{\theta_{1} \leq \theta_{2}\right\}, \\
& Y\left(\theta_{2}\right)<\tilde{\varphi}\left(\theta_{2}, X\left(\theta_{2}\right)\right)+\kappa \text { on } F \cap\left\{\theta_{1}>\theta_{2}\right\} .
\end{aligned}
$$

Since $\left(\theta_{1}, X\left(\theta_{1}\right)\right) \notin B_{\varepsilon / 2}\left(t_{0}, x_{0}\right)$, it follows from the first two inequalities in (3.3.15) that

$$
Y\left(\theta_{1}\right)<\tilde{\varphi}\left(\theta_{1}, X\left(\theta_{1}\right)\right)+\kappa<w\left(\theta_{1}, X\left(\theta_{1}\right)\right) \text { on } F \cap\left\{\theta_{1} \leq \theta_{2}\right\}
$$

On the other hand, since $Y\left(\theta_{2}\right)<\tilde{\varphi}\left(\theta_{2}, X\left(\theta_{2}\right)\right)+\kappa$ on $F \cap\left\{\theta_{1}>\theta_{2}\right\}$ and (3.3.16) holds, $Y\left(\theta_{2}\right)-\tilde{\varphi}\left(\theta_{2}, X\left(\theta_{2}\right)\right) \leq-\varepsilon$ on $F \cap\left\{\theta_{1}>\theta_{2}\right\}$. Observing that $\left(\theta_{2}, X\left(\theta_{2}\right)\right) \in B_{\varepsilon / 2}\left(t_{0}, x_{0}\right)$ 
on $\left\{\theta_{1}>\theta_{2}\right\}$, we get from the last inequality of (3.3.15) that

$$
Y\left(\theta_{2}\right)<\tilde{\varphi}\left(\theta_{2}, X\left(\theta_{2}\right)\right)-\varepsilon<w\left(\theta_{2}, X\left(\theta_{2}\right)\right) \text { on } F \cap\left\{\theta_{1}>\theta_{2}\right\}
$$

From (3.3.18) and (3.3.19), we get that $Y(\theta)<w(\theta, X(\theta))$ on $F$. Therefore, from the fact $w \in \mathbb{U}_{\text {unco }}^{-}$and the definition of $\rho$ on $A^{c}$,

$$
\mathbb{P}(G \mid F)>0
$$

Therefore, $\mathbb{P}\left(G \cap B \cap E_{1}\right) \geq \mathbb{P}(G \cap F)>0>0$.

\subsubsection{Boundary conditions}

In this subsection, we discuss the boundary conditions at $T$. From the definition of the value function $u_{\text {unco }}$, it holds that $u_{\text {unco }}(T, x)=g(x)$ for all $x \in \mathbb{R}^{d}$. However, $u_{\text {unco }}^{+}$and $u_{\text {unco }}^{-}$may not satisfy this boundary condition. Define

$$
\mathbf{N}(t, x, y, p, \psi):=\left\{(r, s): \exists u \in U \text {, s.t. } r=N^{u}(t, x, y, p) \text { and } s \leq \Delta^{u, e}(t, x, y, \psi)\right\}
$$

and $\delta:=\operatorname{dist}\left(0, \mathbf{N}^{c}\right)-\operatorname{dist}(0, \mathbf{N})$, where dist denotes the Euclidean distance. It holds that

$$
0 \in \operatorname{int}(N(t, x, y, p, \psi)) \text { iff } \delta(t, x, y, p, \psi)>0 .
$$

The upper (resp. lower) semi-continuous envelope of $\delta$ is denoted by $\delta^{*}\left(\operatorname{resp} . \delta_{*}\right)$. Let

$$
u_{\text {unco }}^{+}(T-, x)=\limsup _{\left(t<T, x^{\prime}\right) \rightarrow(T, x)} u_{\text {unco }}^{-}\left(t, x^{\prime}\right), \quad u_{\text {unco }}^{-}(T-, x)=\liminf _{\left(t<T, x^{\prime}\right) \rightarrow(T, x)} u_{\text {unco }}^{-}\left(t, x^{\prime}\right) .
$$

The following theorem is an adaptation of the results in $[32,33,12,13]$.

Theorem 3.3.8. Under Assumptions 3.2.1, 3.2.2, 3.3.3 and 3.3.4, $u_{\text {unco }}^{+}(T-, \cdot)$ is a USC viscosity sub-solution of

$$
\min \left\{\varphi(x)-g(x), \delta_{*} \varphi(x)\right\} \leq 0 \text { on } \mathbb{R}^{d}
$$


On the other hand, under Assumptions 3.2.1, 3.2.2 and 3.3.3, $u_{\text {unco }}^{-}(T-, \cdot)$ is an $L S C$ viscosity super-solution of

$$
\min \left\{\varphi(x)-g(x), \delta^{*} \varphi(x)\right\} \geq 0 \text { on } \mathbb{R}^{d}
$$

Proof. Step 1 (The sub-solution property on $\mathbb{D}_{T}$ ). For the sake of contradiction, we assume that for some $x_{0} \in \mathbb{R}^{d}$ and $\varphi \in C^{2}\left(\mathbb{R}^{d}\right)$ satisfying

$$
0=u_{\text {unco }}^{+}\left(T-, x_{0}\right)-\varphi\left(x_{0}\right)=\max _{x \in \mathbb{R}^{d}}\left(u_{\text {unco }}^{+}(T-, x)-\varphi(x)\right),
$$

it holds that

$$
\varphi\left(x_{0}\right)-g\left(x_{0}\right)>2 \eta \text { and } \delta_{*} \varphi\left(x_{0}\right)>2 \eta \text { for some } \eta>0 \text {. }
$$

Let $\left\{w_{k}\right\}_{k=1}^{\infty}$ be a sequence in $\mathbb{U}_{\text {unco }}^{+}$such that $w_{k} \searrow u_{\text {unco }}^{+}$. Set $\tilde{\varphi}(t, x)=\varphi(x)+\iota \mid x-$ $\left.x_{0}\right|^{n_{0}}+\iota \sqrt{T-t}$ for $\iota>0$, where $\iota$ will be fixed later and $n_{0}$ satisfies

$$
\min _{0 \leq t \leq T}\left(\tilde{\varphi}(t, x)-w_{1}(t, x)\right) \rightarrow \infty \text { as }|x| \rightarrow \infty \text { for any } \iota>0
$$

By the lower semi-continuity of $\delta_{*}$ and the upper semi-continuity of $g$, we can find $\iota>0$ and $\varepsilon>0$ such that for all $(t, x) \in[T-\varepsilon, T] \times \bar{B}_{\varepsilon}\left(x_{0}\right)$ and $y \in \mathbb{R}$ such that $|y-\tilde{\varphi}(t, x)| \leq \varepsilon$,

$$
\begin{gathered}
\tilde{\varphi}(t, x)-g(x)>\eta \text { and } \\
\delta_{*}(t, x, y, D \tilde{\varphi}(t, x), \tilde{\varphi}) \geq \eta .
\end{gathered}
$$

By Assumption 3.3.4, the fact that $\delta \geq \delta_{*},(3.3 .21)$ and (3.3.23), we can find a locally Lipschitz map $\hat{\nu}$ such that

$$
\hat{\nu}(t, x, y, D \tilde{\varphi}(t, x)) \in \mathcal{N}_{0, \eta}(t, x, y, \tilde{\varphi}(t, x), \tilde{\varphi})
$$

for all $(t, x, y) \in \mathbb{D} \times R$ s.t. $(t, x) \in[T-\varepsilon, T] \times \bar{B}_{\varepsilon}\left(x_{0}\right)$ and $|y-\tilde{\varphi}(t, x)| \leq \varepsilon$. 
In (3.3.24), we may need to choose smaller values of $\varepsilon, \iota$ and $\eta$. Fix $\iota$. Since $\partial_{t} \tilde{\varphi}(t, x) \rightarrow-\infty$ as $t \rightarrow T$, by the continuity of $\mu_{Y}, \mu_{X}, \sigma_{X}$ and $\nu$,

$$
\begin{gathered}
\mu_{Y}(t, x, y, \hat{\nu}(t, x, y, D \tilde{\varphi}(t, x)))-\mathcal{L}^{\hat{\nu}(t, x, y, D \tilde{\varphi}(t, x))} \tilde{\varphi}(t, x) \geq \eta \text { for all } \\
(t, x, y) \in \mathbb{D} \times R \text { s.t. }(t, x) \in[T-\varepsilon, T] \times \bar{B}_{\varepsilon}\left(x_{0}\right) \text { and }|y-\tilde{\varphi}(t, x)| \leq \varepsilon .
\end{gathered}
$$

Here we may need to shrink $\varepsilon>0$ again. Since $u_{\text {unco }}^{+}$is USC and $\tilde{\varphi}\left(T, x_{0}\right)=$ $u_{\text {unco }}^{+}\left(T-, x_{0}\right)$, there exists $\alpha>0$ such that $\tilde{\varphi}>u_{\text {unco }}^{+}-2 \alpha$ on $[T-\varepsilon, T) \times \bar{B}_{\varepsilon / 2}\left(x_{0}\right)$ after possibly shrinking $\varepsilon$ another time. Since $w_{k} \searrow u_{\text {unco }}^{+}$, there exists $n_{0} \in \mathbb{N}$ such that

$$
\tilde{\varphi}>w_{n_{0}}-\alpha \text { on }[T-\varepsilon, T) \times \bar{B}_{\varepsilon / 2}\left(x_{0}\right) .
$$

Since $\min _{0 \leq t \leq T}\left(\tilde{\varphi}(t, x)-w_{1}(t, x)\right) \rightarrow \infty$ as $|x| \rightarrow \infty$, we can find $R_{0}>\varepsilon$ such that

$$
\tilde{\varphi}>w_{n_{0}}+\varepsilon \text { on } \mathbb{O}:=[T-\varepsilon, T] \times\left(\mathbb{R}^{d}-\bar{B}_{R_{0}}\left(x_{0}\right)\right) \text {. }
$$

Notice that $\tilde{\varphi}(T, \cdot)-u_{\text {unco }}^{+}(T-, \cdot)$ is strictly positive on the compact set $\mathbb{T}^{*}:=$ $\bar{B}_{R_{0}}\left(x_{0}\right)-B_{\varepsilon / 2}\left(x_{0}\right)$. Hence, by the upper semi-continuity of $u_{\text {unco }}^{+}(T-, \cdot)$, there exists $\zeta>0$ such that

$$
\tilde{\varphi}(T, \cdot)>u_{\text {unco }}^{+}(T-, \cdot)+4 \zeta \text { on } \mathbb{T}^{*}
$$

From (3.3.28), we conclude that there exists $\sigma>0$ such that

$$
\tilde{\varphi}>u_{\text {unco }}^{+}+2 \zeta \text { on }[T-\sigma, T) \times \mathbb{T}^{*}
$$

More precisely, if (3.3.29) does not hold for any $\sigma>0$, then there exists a sequence $\left(t_{n}, x_{n}\right) \in \mathbb{D}_{i}$ such that $t_{n} \rightarrow T, x_{n} \in \mathbb{T}^{*}$ and $\tilde{\varphi}\left(t_{n}, x_{n}\right) \leq u_{\text {unco }}^{+}\left(t_{n}, x_{n}\right)+2 \zeta$. The compactness of $\mathbb{T}^{*}$ implies that there is a subsequence of $\left(t_{n}, x_{n}\right)$ which converges to $\left(T, x^{\prime}\right)$ for some $x^{\prime} \in \mathbb{T}^{*}$. By taking the limsup of the above equation over the subsequence, we get $\tilde{\varphi}\left(T, x^{\prime}\right) \leq u_{\text {unco }}^{+}\left(T-, x^{\prime}\right)+2 \zeta$. This contradicts (3.3.28). Therefore, 
(3.3.29) holds.

In (3.3.29), we choose $\sigma<\varepsilon$. By a Dini-type argument, there exists $n_{1} \geq n_{0}$ such that

$$
\tilde{\varphi}>w_{n_{1}}+\zeta \text { on }[T-\sigma, T) \times \mathbb{T}^{*}
$$

Set $w=w_{n_{1}}$. For $\kappa \in(0, \varepsilon \wedge \alpha \wedge \zeta \wedge \eta)$, define

$$
w^{\kappa}:=\left\{\begin{array}{l}
(\tilde{\varphi}-\kappa) \wedge w \text { on }[T-\sigma, T] \times \bar{B}_{\varepsilon}\left(x_{0}\right), \\
w \text { outside }[T-\sigma, T] \times \bar{B}_{\varepsilon}\left(x_{0}\right) .
\end{array}\right.
$$

Since $w(t, x) \geq g(x)$ and (3.3.22) holds, we get that $w^{\kappa}(t, x) \geq g(x)$ for all $(t, x) \in \mathbb{D}$.

We also notice that

$$
w^{\kappa}\left(T, x_{0}\right) \leq \varphi\left(x_{0}\right)-\kappa<u_{\text {unco }}^{+}\left(T-, x_{0}\right) \leq u_{\text {unco }}^{+}\left(T, x_{0}\right)
$$

Using (3.3.24), (3.3.25), (3.3.26), (3.3.27) and (3.3.30) in a manner that is similar to Step 1 in Theorem 3.3.7's proof, we can show that $w^{\kappa}$ is a stochastic super-solution, which contradicts (3.3.31).

Step 2 (The super-solution property on $\mathbb{D}_{T}$ ). We've already proved that $u_{\text {unco }}^{-}(t, x) \geq g(x)$ for $(t, x) \in \mathbb{D}$. Therefore, $u_{\text {unco }}^{-}(T-, x) \geq g(x)$. It remains to prove that $u_{\text {unco }}^{-}(T-, \cdot)$ is a viscosity super-solution of $\delta^{*} \varphi(x) \geq 0$. Let $x_{0} \in \mathbb{R}^{d}$ and $\varphi \in C^{2}\left(\mathbb{R}^{d}\right)$ be such that $0=\left(u_{\text {unco }}^{-}\left(T-, x_{0}\right)-\varphi\left(x_{0}\right)\right)=\min _{\mathbb{R}^{d}}\left(u_{\text {unco }}^{-}(T-, x)-\varphi(x)\right)$. Let $\left(s_{n}, \xi_{n}\right)$ be a sequence in $\mathbb{D}_{i}$ satisfying $\left(s_{n}, \xi_{n}\right) \rightarrow\left(T, x_{0}\right)$ and $u_{\text {unco }}^{-}\left(s_{n}, \xi_{n}\right) \rightarrow$ $u_{\text {unco }}^{-}\left(T-, x_{0}\right)=\varphi\left(x_{0}\right)$. For all $n \in \mathbb{N}, k \geq 0$ and $\iota \geq 0$, define

$$
\varphi_{n}^{k, \iota}(t, x)=\varphi(x)-\iota\left|x-x_{0}\right|^{4}+k \frac{T-t}{\left(T-s_{n}\right)}, \varphi^{\iota}(x)=\varphi(x)-\iota\left|x-x_{0}\right|^{4}
$$

Notice that

$$
\lim _{\iota \rightarrow 0} \lim _{k \rightarrow 0} \limsup _{n \rightarrow \infty} \sup _{(t, x) \in\left[s_{n}, T\right] \times \bar{B}_{1}\left(x_{0}\right)}\left|\varphi_{n}^{k, \iota}(t, x)-\varphi(x)\right|=0 .
$$


Let $\left(t_{n}^{k, \iota}, x_{n}^{k, \iota}\right)$ be the minimizer of $u^{-}-\varphi_{n}^{k, \iota}$ on $\left[s_{n}, T\right] \times \bar{B}_{1}\left(x_{0}\right)$. We claim that for any $k>0$ and $\iota>0$, there exists $N^{k, \iota} \in \mathbb{N}$ such that

$$
s_{n} \leq t_{n}^{k, \iota}<T \text { for all } n \geq N^{k, \iota} \text {, and } x_{n}^{k, \iota} \rightarrow x_{0} \text { as } n \rightarrow \infty \text {. }
$$

We now prove (3.3.32). Since $\left(s_{n}, \xi_{n}\right) \rightarrow\left(T, x_{0}\right)$, we can find $N^{k, \iota} \in \mathbb{N}$ such that for $n \geq N^{k, \iota}$

$$
\left(u_{\text {unco }}^{-}-\varphi_{n}^{k, \iota}\right)\left(s_{n}, \xi_{n}\right)=u_{\text {unco }}^{-}\left(s_{n}, \xi_{n}\right)-\varphi\left(\xi_{n}\right)+\iota\left|\xi_{n}-x_{0}\right|^{4}-\frac{1}{k} \leq-\frac{1}{2 k}<0
$$

On the other hand,

$$
\liminf _{t \uparrow T, x^{\prime} \rightarrow x}\left(u_{\text {unco }}^{-}-\varphi_{n}^{k, \iota}\right)\left(t, x^{\prime}\right)=u_{\text {unco }}^{-}(T-, x)-\varphi(x)+\iota\left|x-x_{0}\right|^{4} \geq 0 \text { for }\left|x-x_{0}\right| \leq 1 .
$$

By (3.3.33) and (3.3.34), the first part of (3.3.32) holds. By an argument similar to Step 4 in Theorem 3.1's proof in [9], we know that the second part of (3.3.32) also holds.

From (3.3.32) and the definition of $\varphi_{n}^{k, \iota}$, we also see that

$$
\varphi_{n}^{k, \iota}\left(t_{n}^{k, \iota}, x_{n}^{k, \iota}\right) \rightarrow u_{\text {unco }}^{-}\left(T-, x_{0}\right)=\varphi\left(x_{0}\right) \text { as } n \rightarrow \infty, \text { then } k \rightarrow 0, \iota \rightarrow 0
$$

By $(3.3 .32),(3.3 .35)$ and the facts that $\liminf _{(t<T, x) \rightarrow\left(T, x_{0}\right)} u_{\text {unco }}^{-}(t, x)=u_{\text {unco }}^{-}\left(T-, x_{0}\right)$ and $u_{\text {unco }}^{-}\left(t_{n}^{k, \iota}, x_{n}^{k, \iota}\right) \leq \varphi_{n}^{k, \iota}\left(t_{n}^{k, \iota}, x_{n}^{k, \iota}\right)$, it holds that $u_{\text {unco }}^{-}\left(t_{n}^{k, \iota}, x_{n}^{k, \iota}\right) \rightarrow u_{\text {unco }}^{-}\left(T-, x_{0}\right)=$ $\varphi\left(x_{0}\right)$ as $n \rightarrow \infty$ then $k \rightarrow 0, \iota \rightarrow 0$. Since for all $k>0, \iota>0$ and $n \geq N^{k, \iota},\left(t_{n}^{k, \iota}, x_{n}^{k, \iota}\right)$ is a local minimizer of $u_{\text {unco }}^{-}-\varphi_{n}^{k, \iota}$ and $t_{n}^{k, \iota}<T$, we get

$$
-\partial_{t} \varphi_{n}^{k, \iota}\left(t_{n}^{k, \iota}, x_{n}^{k, \iota}\right)+H^{*}\left(t_{n}^{k, \iota}, x_{n}^{k, \iota}, u_{\text {unco }}^{-}\left(t_{n}^{k, \iota}, x_{n}^{k, \iota}\right), D \varphi_{n}^{k, \iota}\left(t_{n}^{k, \iota}, x_{n}^{k, \iota}\right), D^{2} \varphi_{n}^{k, \iota}\left(t_{n}^{k, \iota}, x_{n}^{k, \iota}\right)\right) \geq 0
$$

from Theorem 3.3.7. For any $k>0, \iota>0$ and $n \geq N_{n}^{k, \iota}$, from the definition of $H^{*}$, there exists a sequence $\left\{\left(\varepsilon_{m}, \eta_{m}, \Theta_{m}, \varphi_{m}\right)\right\} \subset \mathbb{R}_{+} \times[-1,1] \times \mathbb{D} \times \mathbb{R} \times \mathbb{R}^{d} \times \mathbb{M}^{d} \times C(\mathbb{D})$ 
such that $\left(\varepsilon_{m}, \eta_{m}\right) \rightarrow(0,0)$,

$$
\begin{gathered}
\varphi_{m} \stackrel{\text { u.c. }}{\longrightarrow} \varphi_{n}^{k, \iota}, \Theta_{m} \rightarrow\left(\cdot, u_{\text {unco }}^{-}(\cdot), D \varphi_{n}^{k, \iota}(\cdot), D^{2} \varphi_{n}^{k, \iota}(\cdot)\right)\left(t_{n}^{k, \iota}, x_{n}^{k, \iota}\right), \\
H_{\varepsilon_{m}, \eta_{m}}\left(\Theta_{m}, \varphi_{m}\right) \rightarrow H^{*}\left(\cdot, u_{\text {unco }}^{-}(\cdot), D \varphi_{n}^{k, \iota}(\cdot), D^{2} \varphi_{n}^{k, \iota}(\cdot), \varphi_{n}^{k, \iota}\right)\left(t_{n}^{k, \iota}, x_{n}^{k, \iota}\right)>-\infty .
\end{gathered}
$$

This implies that $\mathcal{N}_{\varepsilon_{m}, \eta_{m}}\left(t_{m}, x_{m}, y_{m}, p_{m}, \varphi_{m}\right) \neq \emptyset$ since $\sup \emptyset=-\infty$. By the definition of $\delta$, it holds that $\delta\left(t_{m}, x_{m}, y_{m}, p_{m}, \varphi_{m}\right) \geq-\sqrt{\varepsilon_{m}^{2}+\eta_{m}^{2}}$. From (3.3.36) and the definition of $\delta^{*}$, we get

$$
\delta^{*}\left(t_{n}^{k, \iota}, x_{n}^{k, \iota}, u_{\text {unco }}^{-}\left(t_{n}^{k, \iota}, x_{n}^{k, \iota}\right), D \varphi_{n}^{k, \iota}\left(t_{n}^{k, \iota}, x_{n}^{k, \iota}\right), \varphi_{n}^{k, \iota}\right) \geq \limsup _{m \rightarrow \infty} \delta\left(t_{m}, x_{m}, y_{m}, p_{m}, \varphi_{m}\right) \geq 0
$$

By the definition of $\Delta^{u, e}$ in the set-valued map $\mathbf{N}$, the equation above implies that

$$
\delta^{*}\left(\cdot, u_{\text {unco }}^{-}(\cdot), D \varphi_{n}^{k, \iota}(\cdot), \varphi^{\iota}\right)\left(t_{n}^{k, \iota}, x_{n}^{k, \iota}\right)=\delta^{*}\left(\cdot, u_{\text {unco }}^{-}(\cdot), D \varphi_{n}^{k, \iota}(\cdot), \varphi_{n}^{k, \iota}\right)\left(t_{n}^{k, \iota}, x_{n}^{k, \iota}\right) \geq 0 .
$$

Note that $\varphi^{\iota} \stackrel{\text { u.c. }}{\longrightarrow} \varphi$ as $\iota \rightarrow 0$. Moreover, for $\iota>0, u_{\text {unco }}^{-}\left(t_{n}^{k, \iota}, x_{n}^{k, \iota}\right) \rightarrow \varphi\left(x_{0}\right)$ and $D \varphi_{n}^{k, \iota}\left(t_{n}^{k, \iota}, x_{n}^{k, \iota}\right) \rightarrow D \varphi\left(x_{0}\right)$ as $n \rightarrow \infty$ then $k \rightarrow 0$. Taking the limsup of (3.3.37) by first sending $n \rightarrow \infty$ then $k \rightarrow 0$ and $\iota \rightarrow 0$, we have

$$
\delta^{*} \varphi\left(x_{0}\right)=\delta^{*} \varphi\left(T, x_{0}, \varphi\left(x_{0}\right), D \varphi\left(x_{0}\right), \varphi\right) \geq 0
$$

from the upper semi-continuity of $\delta^{*}$,

\subsubsection{Verification by comparison}

We now carry out the verification for non-smooth functions assuming the comparison principle as in the previous chapter.

Assumption 3.3.9. Let $H=H_{*}$. Assume that $H=H^{*}$ on the set $\{H<\infty\}$ and that there exists an LSC function $G: \mathbb{D} \times \mathbb{R} \times \mathbb{R}^{d} \times \mathbb{M}^{d} \times C(\mathbb{D}) \rightarrow \mathbb{R}$ such that

$$
\begin{aligned}
& \text { (a) } H(t, x, y, p, A, \varphi)<\infty \Longrightarrow G(t, x, y, p, A, \varphi) \leq 0 \\
& \text { (b) } G(t, x, y, p, A, \varphi)<0 \Longrightarrow H(t, x, y, p, A, \varphi)<\infty
\end{aligned}
$$


Proposition 3.3.10. Under Assumptions 3.2.1, 3.2.2, 3.3.3, 3.3.4 and 3.3.9, $u_{\text {unco }}^{+}$ (resp. $u_{\text {unco }}^{-}$) is a USC (resp. an LSC) viscosity sub-solution (resp. super-solution) of

$$
\min \left\{\varphi(t, x)-g(x), \max \left\{-\partial_{t} \varphi(t, x)+H \varphi(t, x), G \varphi(t, x)\right\}\right\}=0 \text { on } \mathbb{D}_{i} .
$$

Proof. This proposition follows from similar arguments to those in Proposition 2.5.2.

Assumption 3.3.11. Assume that $\delta^{*}=\delta_{*}$ and a comparison principle holds between USC sub-solutions and LSC super-solutions for

$$
\min \{\varphi(x)-g(x), \delta \varphi(x)\}=0 \text { on } \mathbb{R}^{d} .
$$

In the presence of jumps, it is nontrivial to check this assumption. When there are no jumps in the controlled processes, the comparison principle can be proved in certain classes of functions (see the discussion above Assumption 2.2 in [14]). Also, in Section 3.3.4, $\delta$ drops out in the corresponding PDE and there are comparison results available for fully non-linear equations with jumps (see [1]).

Lemma 3.3.12. Under Assumptions 3.2.1, 3.2.2, 3.3.3, 3.3.4, 3.3.9 and 3.3.11, $u_{\text {unco }}^{-}(T-, \cdot)=u_{\text {unco }}^{+}(T-, \cdot)=\hat{g}(\cdot)$, where $\hat{g}$ is the unique continuous viscosity solution to $(3.3 .38)$.

Proof. It follows from their definitions that $u_{\text {unco }}^{-} \leq u_{\text {unco }}^{+}$. Since $u_{\text {unco }}^{+}$is USC and $u_{\text {unco }}^{-}$is LSC, then

$$
u_{\text {unco }}^{-}(T-, x)=\liminf _{\left(t<T, x^{\prime}\right) \rightarrow(T, x)} u_{\text {unco }}^{-}\left(t, x^{\prime}\right) \leq \limsup _{\left(t<T, x^{\prime}\right) \rightarrow(T, x)} u_{\text {unco }}^{+}\left(t, x^{\prime}\right)=u_{\text {unco }}^{+}(T-, x) .
$$

Moreover, $u_{\mathrm{unco}}^{+}(T-, \cdot)$ is a viscosity sub-solution and $u_{\text {unco }}^{-}(T-, \cdot)$ is a viscosity supersolution to (3.3.38) due to Theorem 3.3.8. Therefore, the claim holds by Assumption 3.3.11. 
Theorem 3.3.13. Suppose that there is a comparison principle for

$$
\min \left\{\varphi(t, x)-g(x), \max \left\{-\partial_{t} \varphi(t, x)+H \varphi(t, x), G \varphi(t, x)\right\}\right\}=0 \text { on } \mathbb{D}_{i}
$$

and that Assumptions 3.2.1, 3.2.2, 3.3.3, 3.3.4, 3.3.9 and 3.3.11 hold. Then there exists a unique continuous viscosity solution $V_{\text {unco }}$ to (3.3.39) with terminal condition $V_{\text {unco }}(T, x)=\hat{g}(x)$ and $u_{\text {unco }}(t, x)=u_{\text {unco }}^{-}(t, x)=u_{\text {unco }}^{+}(t, x)=V_{\text {unco }}(t, x)$ for $(t, x) \in$ $\mathbb{D}_{i}$

Proof. Define

$$
\hat{u}_{\text {unco }}^{+}(t, x):= \begin{cases}u_{\text {unco }}^{+}(t, x), & (t, x) \in \mathbb{D}_{i}, \\ \hat{g}(x), & t=T, x \in \mathbb{R}^{d} .\end{cases}
$$

and

$$
\hat{u}_{\text {unco }}^{-}(t, x):= \begin{cases}u_{\text {unco }}^{-}(t, x), & (t, x) \in \mathbb{D}_{i}, \\ \hat{g}(x), & t=T, x \in \mathbb{R}^{d} .\end{cases}
$$

From Proposition 3.3.10, $\hat{u}_{\text {unco }}^{-}$is an LSC viscosity super-solution and $\hat{u}_{\text {unco }}^{+}$is a USC viscosity sub-solution of (3.3.39). Since $\hat{u}_{\text {unco }}^{+}(T, \cdot)=\hat{u}_{\text {unco }}^{-}(T, \cdot), \hat{u}_{\text {unco }}^{+} \leq \hat{u}_{\text {unco }}^{-}$on $\mathbb{D}$ by comparison. Hence, $\hat{u}_{\text {unco }}^{+}=\hat{u}_{\text {unco }}^{-}$on $\mathbb{D}$ from (3.3.1). Define $V_{\text {unco }}:=\hat{u}_{\text {unco }}^{+}=\hat{u}_{\text {unco }}^{-}$. It is a continuous viscosity solution of (3.3.39) satisfying $V_{\text {unco }}(T, x)=\hat{g}(x)$. Uniqueness follows directly from the comparison principle.

\subsubsection{Stochastic controller-stopper game as a stochastic target problem with a non- cooperative stopper}

In this subsection, we show how the HJB equation associated to a (semi) controllerstopper game can be deduced from a stochastic target problem with a non-cooperative stopper. Given a bounded continuous function $g: \mathbb{R}^{d} \rightarrow \mathbb{R}$, we define a (semi) controller-stopper game by

$$
\mathbf{u}_{\text {unco }}(t, x):=\inf _{\nu \in \mathcal{U}^{t}} \sup _{\rho \in \mathcal{T}_{t}} \mathbb{E}\left[g\left(X_{t, x}^{\nu}(\rho)\right)\right]
$$


We follow the setup of Section 3.2 with one exception: $\mathcal{U}^{t}$ is the collection of all the $\mathbb{F}^{t}$-predictable processes in $\mathbb{L}^{2}\left(\Omega \times[0, T], \mathcal{F} \otimes \mathcal{B}[0, T], \mathbb{P} \otimes \lambda_{L} ; U\right)$, where $U \subset \mathbb{R}^{d}$ and $X$ follows the SDE

$d X(s)=\mu_{X}(s, X(s), \nu(s)) d s+\sigma_{X}(s, X(s), \nu(s)) d W_{s}+\int_{E} \beta(s, X(s-), \nu(s), e) \lambda(d s, d e)$.

To convert the controller-stopper game to its stochastic target counterpart, we need the following lemma, whose proof relies crucially on the technical result Lemma 3.5.6.

Lemma 3.3.14. Suppose Assumptions 3.2.1 and 3.2.2 hold. Define a stochastic target problem as follows:

$$
\begin{array}{r}
u_{\text {unco }}(t, x):=\inf \left\{y \in \mathbb{R}: \exists(\nu, \alpha, \gamma) \in \mathcal{U}^{t} \times \mathcal{A}^{t} \times \Gamma_{\text {unco }}^{t}\right. \\
\text { s.t. } \left.Y_{t, y}^{\alpha, \gamma}(\rho) \geq g\left(X_{t, x}^{\nu}(\rho)\right) \text { for all } \rho \in \mathcal{T}_{t}\right\},
\end{array}
$$

where

$$
Y_{t, y}^{\alpha, \gamma}(\cdot):=y+\int_{t}^{\cdot} \alpha^{\top}(s) d W_{s}+\int_{t}^{\cdot} \int_{E} \gamma^{\top}(s, e) \tilde{\lambda}(d s, d e) .
$$

Here, $\mathcal{A}^{t}$ and $\Gamma_{\text {unco }}^{t}$ are the collections of $\mathbb{R}^{d}$-valued and $\mathbb{L}^{2}\left(E, \mathcal{E}, \hat{m} ; \mathbb{R}^{I}\right)$-valued processes, respectively, satisfying the admissibility conditions in Section 3.2. Then $u_{\text {unco }}=\mathbf{u}_{\text {unco }}$ on $\mathbb{D}$.

Proof. For fixed $\nu \in \mathcal{U}^{t}$, let

$$
A^{\nu}(s):=\operatorname{esssup}_{\tau \in \mathcal{T}_{s}} \mathbb{E}\left[g\left(X_{t, x}^{\nu}(\tau)\right) \mid \mathcal{F}_{s}\right], s \geq t
$$

Then $A^{\nu}$ is the snell envelope (starting at $t$ ) of $g\left(X_{t, x}^{\nu}\right)$ and thus a super-martingale. Moreover,

$$
\underset{\tau \in \mathcal{T}_{t}}{\operatorname{esssup}} \mathbb{E}\left[G^{\nu}(\tau) \mid \mathcal{F}_{t}\right]+A^{\nu}(\rho)-A^{\nu}(t) \geq G^{\nu}(\rho) \text { for all } \rho \in \mathcal{T}_{t}
$$

By Doob-Meyer Decomposition Theorem, $A_{s}^{\nu}=M_{s}^{\nu}-C_{s}^{\nu}$ for $s \in[t, T]$, where $M^{\nu}$ is a martingale on $[t, T]$ and $C^{\nu}$ is an increasing adapted process with $C_{t}^{\nu}=0$. Therefore,

$$
\underset{\tau \in \mathcal{T}_{t}}{\operatorname{esssup}} \mathbb{E}\left[G^{\nu}(\tau) \mid \mathcal{F}_{t}\right]+M^{\nu}(\rho)-M^{\nu}(t) \geq G^{\nu}(\rho) \text { for all } \rho \in \mathcal{T}_{t}
$$


Denote $\mathcal{M}_{\text {unco }}=\left\{M^{\nu}: \nu \in \mathcal{U}^{t}\right\}$. In view of Lemma 3.5.6, it suffices to check that

$$
\mathcal{M}_{\text {unco }} \subset \mathcal{M}:=\left\{Y_{t, y}^{\alpha, \gamma}(\cdot): y \in \mathbb{R}, \alpha \in \mathcal{A}^{t}, \gamma \in \Gamma_{\text {unco }}^{t}\right\} .
$$

In fact, by the martingale representation theorem, for any $\nu \in \mathcal{U}^{t}, M^{\nu}$ can be represented in the form of $Y_{t, y}^{\alpha, \gamma}$ for some $\alpha \in \mathcal{A}^{t}$ and $\gamma \in \Gamma_{0}^{t}$, where $\Gamma_{0}^{t}$ is the collection of $\mathbb{L}^{2}\left(E, \mathcal{E}, \hat{m} ; \mathbb{R}^{I}\right)$-valued processes satisfying all of the admissibility conditions except for (3.2.2). Assume, contrary to (3.3.40), that there exists $\nu_{0} \in \mathcal{U}^{t}$ such that

$$
\mathcal{M}^{\nu_{0}}(\cdot)=y+\int_{t} \alpha_{0}^{\top}(s) d W_{s}+\int_{t}^{\cdot} \int_{E} \gamma_{0}^{\top}(s, e) \tilde{\lambda}(d s, d e)
$$

for some $y \in \mathbb{R}, \alpha_{0} \in \mathcal{A}^{t}$ and $\gamma_{0} \in \Gamma_{0}^{t}$, but (3.2.2) does not hold. This means that for $K>2\|g\|_{\infty}$, there exists $\tau_{0} \in \mathcal{T}_{t}$ such that

$$
\mathbb{P}\left(\int_{E} \gamma_{0}^{\top}\left(\tau_{0}, e\right) \lambda\left(\left\{\tau_{0}\right\}, d e\right) \leq-K\right)>0
$$

Therefore,

$$
M^{\nu_{0}}\left(\tau_{0}\right)-M^{\nu_{0}}\left(\tau_{0}-\right)=\int_{E} \gamma_{0}^{\top}\left(\tau_{0}, e\right) \lambda\left(\left\{\tau_{0}\right\}, d e\right) \leq-K \text { with positive probability }
$$

which further implies that

$$
A^{\nu_{0}}\left(\tau_{0}\right)-A^{\nu_{0}}\left(\tau_{0}-\right) \leq-K \text { with positive probability. }
$$

This contradicts the fact that $A^{\nu_{0}}$ is (strictly) bounded by $\frac{K}{2}$.

Let $\mathbf{H}^{*}$ be the USC envelope of the LSC map $\mathbf{H}: \mathbb{D} \times \mathbb{R}^{d} \times \mathbb{M}^{d} \times C(\mathbb{D}) \rightarrow \mathbb{R}$ defined by

$$
\begin{gathered}
\mathbf{H}:(t, x, p, A, \varphi) \rightarrow \sup _{u \in U}\left\{-I[\varphi](t, x, u)-\mu_{X}^{\top}(t, x, u) p-\frac{1}{2} \operatorname{Tr}\left[\sigma_{X} \sigma_{X}^{\top}(t, x, u) A\right]\right\}, \\
\quad \text { where } I[\varphi](t, x, u)=\sum_{1 \leq i \leq I} \int_{E}\left(\varphi\left(t, x+\beta_{i}(t, x, u, e)\right)-\varphi(t, x)\right) m_{i}(d e) .
\end{gathered}
$$


Theorem 3.3.15. Under Assumptions 3.2.1 and 3.2.2, $u_{\text {unco }}^{+}$is a USC viscosity sub-solution of

$$
\min \left\{\varphi(t, x)-g(x),-\partial_{t} \varphi(t, x)+\mathbf{H} \varphi(t, x)\right\} \leq 0 \text { on } \mathbb{D}_{i}
$$

and $u_{\text {unco }}^{+}(T-, x) \leq g(x)$ for all $x \in \mathbb{R}^{d}$. On the other hand, $u_{\text {unco }}^{-}$is an LSC viscosity super-solution of

$$
\min \left\{\varphi(t, x)-g(x),-\partial_{t} \varphi(t, x)+\mathbf{H}^{*} \varphi(t, x)\right\} \geq 0 \text { on } \mathbb{D}_{i}
$$

and $u_{\text {unco }}^{-}(T-, \cdot) \geq g(x)$ for all $x \in \mathbb{R}^{d}$.

Proof. It is easy to check Assumption 3.3.4 for the stochastic target problem. Since $g$ is bounded, we can check that all of the assumptions in the Section 3.5 are satisfied, which implies that Assumption 3.3.3 holds. From Theorem 3.3.7, $u_{\text {unco }}^{+}$is a USC viscosity sub-solution of

$$
\min \left\{\varphi(t, x)-g(x),-\partial_{t} \varphi(t, x)+H_{*} \varphi(t, x)\right\} \leq 0 \text { on } \mathbb{D}_{i}
$$

and $u_{\text {unco }}^{-}$is an LSC viscosity super-solution of

$$
\min \left\{\varphi(t, x)-g(x),-\partial_{t} \varphi(t, x)+H^{*} \varphi(t, x)\right\} \geq 0 \text { on } \mathbb{D}_{i}
$$

From Proposition 3.1 in [13], $H^{*} \leq \mathbf{H}^{*}$ and $H_{*} \geq \mathbf{H}$. This implies that the viscosity properties in the parabolic interior hold. Note that $\delta=\operatorname{dist}\left(0, \mathbf{N}^{c}\right)-\operatorname{dist}(0, \mathbf{N})$, where

$$
\begin{aligned}
& \mathbf{N}(t, x, y, p, \varphi)= \\
& \left\{(q, s): \exists(u, a, r) \in U \times \mathbb{R}^{d} \times \mathbb{L}^{2}\left(E, \mathcal{E}, \hat{m} ; \mathbb{R}^{I}\right) \text { s.t. } q=a-\sigma_{X}^{\top}(t, x, u) p\right. \\
& \text { and } \left.s \leq \min _{1 \leq i \leq I}\left\{r_{i}(e)-\varphi\left(t, x+\beta_{i}(t, x, u, e)\right)+\varphi(t, x)\right\} \hat{m}-\text { a.s. } e \in E\right\} .
\end{aligned}
$$

Obviously, $\mathbf{N}=\mathbb{R}^{d} \times \mathbb{R}$. Therefore, $\delta=\infty$ and the boundary conditions hold. 
The following two corollaries show that $\mathbf{u}_{\text {unco }}$ is the unique viscosity solution to its associated HJB equation. We omit the proof, since it is the same as the proofs of Proposition 3.3.10 and Theorem 3.3.13.

Corollary 3.3.16. Suppose that Assumptions 3.2.1 and 3.2.2 hold, $\mathbf{H}=\mathbf{H}^{*}$ on $\{\mathbf{H}<\infty\}$ and there exists an LSC function $\mathbf{G}: \mathbb{D} \times \mathbb{R} \times \mathbb{R}^{d} \times \mathbb{M}^{d} \times C(\mathbb{D}) \rightarrow \mathbb{R}$ such that

$$
\begin{aligned}
& \text { (a) } \mathbf{H}(t, x, y, p, M, \varphi)<\infty \Longrightarrow \mathbf{G}(t, x, y, p, M, \varphi) \leq 0, \\
& \text { (b) } \mathbf{G}(t, x, y, p, M, \varphi)<0 \Longrightarrow \mathbf{H}(t, x, y, p, M, \varphi)<\infty .
\end{aligned}
$$

Then $u_{\text {unco }}^{+}\left(\right.$resp. $\left.u_{\text {unco }}^{-}\right)$is a USC (resp. an LSC) viscosity sub-solution (resp. supersolution) of

$$
\min \left\{\varphi(t, x)-g(x), \max \left\{-\partial_{t} \varphi(t, x)+\mathbf{H} \varphi(t, x), \mathbf{G} \varphi(t, x)\right\}\right\}=0 \text { on } \mathbb{D}_{i}
$$

Corollary 3.3.17. Suppose that all the assumptions in Corollary 3.3.16 hold. Then $u_{\text {unco }}^{+}(T-, x)=u_{\text {unco }}^{-}(T-, x)=g(x)$. Moreover, if the comparison principle holds for

$$
\min \left\{\varphi(t, x)-g(x), \max \left\{-\partial_{t} \varphi(t, x)+\mathbf{H} \varphi(t, x), \mathbf{G} \varphi(t, x)\right\}\right\}=0 \text { on } \mathbb{D}_{i}
$$

Then $u_{\text {unco }}\left(=\mathbf{u}_{\text {unco }}\right)$ is the unique continuous viscosity solution with $u_{\text {unco }}(T, x)=$ $g(x)$

\subsection{The stochastic target problem with a cooperative stopper}

Definition 3.4.1 (Stochastic Super-solutions). A continuous function $w: \mathbb{D} \rightarrow \mathbb{R}$ is called a stochastic super-solution if

1. $w(t, x) \geq g(x)$ and for some $C>0$ and $n \in \mathbb{N},|w(t, x)| \leq C\left(1+|x|^{n}\right)$ for all $(t, x) \in \mathbb{D}$.

2. Given $(t, x, y) \in \mathbb{D} \times \mathbb{R}$, for any $\tau \in \mathcal{T}_{t}, \rho \in \mathcal{T}_{\tau}$ and $\nu \in \mathcal{U}_{\text {co }}^{t}$, we have

$$
\mathbb{P}(Y(\rho)>w(\rho, X(\rho)) \mid B)>0
$$


for any $B \subset\{Y(\tau)>w(\tau, X(\tau))\}$ satisfying $B \in \mathcal{F}_{\tau}^{t}$ and $\mathbb{P}(B)>0$. Here, $X:=X_{t, x}^{\nu}$ and $Y:=Y_{t, x, y}^{\nu}$

Definition 3.4.2 (Stochastic Sub-solutions). A continuous function $w: \mathbb{D} \rightarrow \mathbb{R}$ is called a stochastic sub-solution if

1. $w(T, x) \leq g(x)$ for all $x \in \mathbb{R}^{d}$ and for some $C>0$ and $n \in \mathbb{N},|w(t, x)| \leq$ $C\left(1+|x|^{n}\right)$ for all $(t, x) \in \mathbb{D}$.

2. Given $(t, x, y) \in \mathbb{D} \times \mathbb{R}$, for any $\tau \in \mathcal{T}_{t}$ and $\nu \in \mathcal{U}_{\text {co }}^{t}$, there exist $\rho \in \mathcal{T}_{\tau}$ and $\tilde{\nu} \in \mathcal{U}_{\text {co }}^{t}$ such that

$$
Y(\rho) \leq g(X(\rho)) \mathbb{P} \text {-a.s. on }\{Y(\tau) \leq w(\tau, X(\tau))\}
$$

where $X:=X_{t, x}^{\nu \otimes_{\tau} \tilde{\nu}}$ and $Y:=Y_{t, x, y}^{\nu \otimes_{\tau} \tilde{\nu}}$.

Denote the sets of stochastic super-solutions and sub-solutions by $\mathbb{U}_{\text {co }}^{+}$and $\mathbb{U}_{\text {co }}^{-}$, respectively.

Assumption 3.4.3. $\mathbb{U}_{c o}^{+}$and $\mathbb{U}_{c o}^{-}$are not empty.

When the above assumption holds, we can check from the definitions of stochastic solutions that

$$
u_{\mathrm{co}}^{-}:=\sup _{w \in \mathbb{U}_{\mathrm{co}}^{-}} w \leq u_{\mathrm{co}} \leq \inf _{w \in \mathbb{U}_{\mathrm{co}}^{+}} w=: u_{\mathrm{co}}^{+}
$$

\subsubsection{Viscosity property in $\mathbb{D}_{i}$}

In the rest of this subsection, we state and prove the theorem which characterizes $u_{\text {co }}^{-}$as a viscosity super-solution of

$$
\min \left\{\varphi(t, x)-g(x),-\partial_{t} \varphi(t, x)+F^{*} \varphi(t, x)\right\} \geq 0 \text { in } \mathbb{D}_{i}
$$

and $u_{\mathrm{co}}^{+}$as a viscosity sub-solution of

$$
\min \left\{\varphi(t, x)-g(x),-\partial_{t} \varphi(t, x)+F_{*} \varphi(t, x)\right\} \leq 0 \text { in } \mathbb{D}_{i}
$$


The boundary conditions will be discussed in Theorem 3.4.8. Before we state the main result, we impose a regularity assumption on the set-valued map $\mathcal{M}_{0,-\eta}(\cdot, \psi)$, which is crucial to the super-solution property of $u_{\mathrm{co}}^{-}$.

Assumption 3.4.4. For $\psi \in C(\mathbb{D}), \eta>0$, let $B$ be a subset of $\mathbb{D} \times \mathbb{R} \times \mathbb{R}^{d}$ such that $\mathcal{M}_{0,-\eta}(\cdot, \psi) \neq \emptyset$ on $B$. Then for every $\varepsilon>0,\left(t_{0}, x_{0}, y_{0}, p_{0}\right) \in \operatorname{Int}(B)$ and $u_{0} \in$ $\mathcal{M}_{0,-\eta}\left(t_{0}, x_{0}, y_{0}, p_{0}, \psi\right)$, there exists an open neighborhood $B^{\prime}$ of $\left(t_{0}, x_{0}, y_{0}, p_{0}\right)$ and a locally Lipschitz continuous map $\hat{\nu}$ defined on $B^{\prime}$ such that $\left\|\hat{\nu}\left(t_{0}, x_{0}, y_{0}, p_{0}\right)-u_{0}\right\|_{U} \leq \varepsilon$ and $\hat{\nu}(t, x, y, p) \in \mathcal{M}_{0,-\eta}(t, x, y, p, \psi)$.

Lemma 3.4.5. $\mathbb{U}_{c o}^{+}$and $\mathbb{U}_{c o}^{-}$are closed under pairwise minimization and maximization, respectively.

Lemma 3.4.6. There exists a non-increasing sequence $\left\{w_{n}\right\}_{n=1}^{\infty} \subset \mathbb{U}_{c o}^{+}$such that $w_{n} \searrow u_{c o}^{+}$and a non-decreasing sequence $\left\{v_{n}\right\}_{n=1}^{\infty} \subset \mathbb{U}_{c o}^{-}$such that $v_{n} \nearrow u_{c o}^{-}$.

Theorem 3.4.7. Under Assumptions 3.2.1, 3.2.2, 3.4.3 and 3.4.4, $u_{c o}^{+}$is a USC viscosity sub-solution of (3.4.3). On the other hand, under Assumptions 3.2.1, 3.2.2 and 3.4.3, $u_{c o}^{-}$is an LSC viscosity super-solution of (3.4.2).

Proof. Step 1 ( $u_{\text {co }}^{+}$is a viscosity sub-solution). Let $\left(t_{0}, x_{0}\right) \in \mathbb{D}_{i}$ satisfy $0=$ $\left(u_{\mathrm{co}}^{+}-\varphi\right)\left(t_{0}, x_{0}\right)=\max _{\mathbb{D}_{i}}\left(u_{\mathrm{co}}^{+}-\varphi\right)$ for some $\varphi \in C^{1,2}(\mathbb{D})$. For the sake of contradiction, assume that

$$
2 \eta:=-\partial_{t} \varphi\left(t_{0}, x_{0}\right)+F^{*} \varphi\left(t_{0}, x_{0}\right)>0 \text { and } \varphi\left(t_{0}, x_{0}\right)>g\left(x_{0}\right)
$$

Let $\left\{w_{k}\right\}_{k=1}^{\infty}$ be a sequence in $\mathbb{U}_{\text {co }}^{+}$such that $w_{k} \searrow u_{\text {co }}^{+}$. Let $\tilde{\varphi}(t, x):=\varphi(t, x)+\iota \mid x-$ $\left.x_{0}\right|^{n_{0}}$, where we choose $n_{0} \geq 2$ such that for all $\iota>0$,

$$
\min _{0 \leq t \leq T}\left(\tilde{\varphi}(t, x)-w_{1}(t, x)\right) \rightarrow \infty \text { and } \min _{0 \leq t \leq T} \tilde{\varphi}(t, x) \rightarrow \infty \text { as }|x| \rightarrow \infty
$$


By (3.4.4), the upper semi-continuity of $F^{*}$ and the fact that $\tilde{\varphi} \stackrel{\text { u.c. }}{\longrightarrow} \varphi$ as $\iota \rightarrow 0$, we can find $\varepsilon>0, \eta>0$ and $\iota>0$ such that

$$
\begin{gathered}
\varphi(t, x)>g(x)+\varepsilon \\
\mu_{Y}(t, x, y, u)-\mathscr{L}^{u} \tilde{\varphi}(t, x) \geq \eta \text { for all } u \in \mathcal{M}_{\varepsilon, \eta}(t, x, y, D \tilde{\varphi}(t, x), \tilde{\varphi}) \\
\text { and }(t, x, y) \in \mathbb{D} \times \mathbb{R} \text { s.t. }(t, x) \in B_{\varepsilon}\left(t_{0}, x_{0}\right) \text { and }|y-\tilde{\varphi}(t, x)| \leq \varepsilon
\end{gathered}
$$

Fix $\iota$. Note that $\left(t_{0}, x_{0}\right)$ is still a strict maximizer of $u_{\mathrm{co}}^{+}-\tilde{\varphi}$. Since (3.4.5) holds, there exists $R_{0}>\varepsilon$ such that

$$
\tilde{\varphi}>w_{1}+\varepsilon \geq w_{k}+\varepsilon \text { on } \mathbb{O}:=\mathbb{D}-[0, T] \times \bar{B}_{R_{0}}\left(x_{0}\right) .
$$

On the compact set $\mathbb{T}:=[0, T] \times \bar{B}_{R_{0}}\left(x_{0}\right)-B_{\varepsilon / 2}\left(t_{0}, x_{0}\right)$, we know that $\tilde{\varphi}>u_{\text {co }}^{+}$and the minimum of $\tilde{\varphi}-u_{\mathrm{co}}^{+}$is attained since $u_{\mathrm{co}}^{+}$is USC. Therefore, $\tilde{\varphi}>u_{\mathrm{co}}^{+}+2 \alpha$ on $\mathbb{T}$ for some $\alpha>0$. By a Dini-type argument, for large enough $n$, we have $\tilde{\varphi}>w_{n}+\alpha$ on $\mathbb{T}$ and $\tilde{\varphi}>w_{n}-\varepsilon$ on $\bar{B}_{\varepsilon / 2}\left(t_{0}, x_{0}\right)$. For simplicity, fix such an $n$ and set $w=w_{n}$. In short,

$$
\tilde{\varphi}>w+\varepsilon \text { on } \mathbb{O}, \tilde{\varphi}>w+\alpha \text { on } \mathbb{T} \text { and } \tilde{\varphi}>w-\varepsilon \text { on } \bar{B}_{\varepsilon / 2}\left(t_{0}, x_{0}\right)
$$

For $\kappa \in(0, \alpha \wedge \varepsilon)$, define

$$
w^{\kappa}:=\left\{\begin{array}{l}
(\tilde{\varphi}-\kappa) \wedge w \text { on } \bar{B}_{\varepsilon}\left(t_{0}, x_{0}\right), \\
w \text { outside } \bar{B}_{\varepsilon}\left(t_{0}, x_{0}\right) .
\end{array}\right.
$$

Noticing that $w^{\kappa}\left(t_{0}, x_{0}\right) \leq \tilde{\varphi}\left(t_{0}, x_{0}\right)-\kappa<u_{\mathrm{co}}^{+}\left(t_{0}, x_{0}\right)$, we will obtain a contradiction if we show that $w^{\kappa} \in \mathbb{U}_{\mathrm{co}}^{+}$. Obviously, $w^{\kappa}$ is continuous, has polynomial growth in $x$ and $w^{\kappa}(t, x) \geq g(x)$ for all $(t, x) \in \mathbb{D}$. Fix $(t, x, y) \in \mathbb{D}_{i} \times \mathbb{R}, \nu \in \mathcal{U}_{\text {co }}^{t}, \tau \in \mathcal{T}_{t}$ and $\rho \in \mathcal{T}_{\tau}$. Let $X=X_{t, x}^{\nu}, Y=Y_{t, x, y}^{\nu}$ and $\theta=\theta_{1} \wedge \theta_{2}$, where

$$
\begin{aligned}
& \theta_{1}:=\inf \left\{s \in[\tau, T]:(s, X(s)) \notin B_{\varepsilon / 2}\left(t_{0}, x_{0}\right)\right\} \wedge T, \\
& \theta_{2}:=\inf \{s \in[\tau, T]:|Y(s)-\tilde{\varphi}(s, X(s))| \geq \varepsilon\} \wedge T .
\end{aligned}
$$


Since $X$ and $Y$ are càdlàg processes, we know that $\theta \in \mathcal{T}_{\tau}$. We know the following also hold:

$$
\begin{aligned}
& \left(\theta_{1}, X\left(\theta_{1}\right)\right) \notin B_{\varepsilon / 2}\left(t_{0}, x_{0}\right), \quad\left|Y\left(\theta_{2}\right)-\tilde{\varphi}\left(\theta_{2}, X\left(\theta_{2}\right)\right)\right| \geq \varepsilon \\
& \left(\theta_{1}, X\left(\theta_{1}-\right)\right) \in \bar{B}_{\varepsilon / 2}\left(t_{0}, x_{0}\right), \quad\left|Y\left(\theta_{2}-\right)-\tilde{\varphi}\left(\theta_{2}, X\left(\theta_{2}-\right)\right)\right| \leq \varepsilon .
\end{aligned}
$$

It now suffices to show that

$$
\mathbb{P}\left(Y(\rho)>w^{\kappa}(\rho, X(\rho)) \mid B\right)>0
$$

for $B \subset\left\{Y(\tau)>w^{\kappa}(\tau, X(\tau))\right\}$ satisfying $B \in \mathcal{F}_{\tau}^{t}$ and $\mathbb{P}(B)>0$. Let $A=$ $\left\{w^{\kappa}(\tau, X(\tau))=w(\tau, X(\tau))\right\}$ and set

$$
\begin{gathered}
E=\left\{Y(\tau)>w^{\kappa}(\tau, X(\tau))\right\}, \quad E_{0}=E \cap A, \quad E_{1}=E \cap A^{c}, \\
G=\left\{Y(\rho)>w^{\kappa}(\rho, X(\rho)\}, \quad G_{0}=\{Y(\rho)>w(\rho, X(\rho)\} .\right.
\end{gathered}
$$

Then $E=E_{0} \cup E_{1}, E_{0} \cap E_{1}=\emptyset$ and $G_{0} \subset G$. To prove that $w^{\kappa} \in \mathbb{U}_{\text {co }}^{+}$, it suffices to show that $\mathbb{P}(G \cap B)>0$. As in [33], we will show

$$
\mathbb{P}\left(B \cap E_{0}\right)>0 \Longrightarrow \mathbb{P}\left(G \cap B \cap E_{0}\right)>0, \mathbb{P}\left(B \cap E_{1}\right)>0 \Longrightarrow \mathbb{P}\left(G \cap B \cap E_{1}\right)>0
$$

This, together with the facts $\mathbb{P}(B)=\mathbb{P}\left(B \cap E_{0}\right)+\mathbb{P}\left(B \cap E_{1}\right)>0$ and $\mathbb{P}(G \cap B)=$ $\mathbb{P}\left(G \cap B \cap E_{0}\right)+\mathbb{P}\left(G \cap B \cap E_{1}\right)$, implies that $\mathbb{P}(G \cap B)>0$.

(i)Assume that $\mathbb{P}\left(B \cap E_{0}\right)>0$. Since $B \cap E_{0} \subset\{Y(\tau)>w(\tau, X(\tau))\}$ and $B \cap E_{0} \in \mathcal{F}_{\tau}^{t}, \mathbb{P}\left(G \mid B \cap E_{0}\right)>0$ from the fact $w \in \mathbb{U}_{\mathrm{co}}^{+}$. This further implies that $\mathbb{P}\left(G \cap B \cap E_{0}\right)>0$.

(ii) Assume that $\mathbb{P}\left(B \cap E_{1}\right)>0$. Let $\Gamma(s):=Y(s)-\tilde{\varphi}(s, X(s))+\kappa$. From similar arguments to those in Step 2.1 of Theorem 2.3.3's proof, we know that $\Gamma L$ is a submartingale on $[\tau, \theta]$. Since $\Gamma(\tau) L(\tau)>0$ on $B \cap E_{1}$, the sub-martingale property implies that there exists $F \subset B \cap E_{1}$ such that $F \in \mathcal{F}_{\tau}^{t}$ and $\Gamma(\theta \wedge \rho) L(\theta \wedge \rho)>0$ on 
$F$. The non-negativity of $L$ then yields $\Gamma(\theta \wedge \rho)>0$ on $F$. Therefore,

$$
\begin{gathered}
Y\left(\theta_{1}\right)>\tilde{\varphi}\left(\theta_{1}, X\left(\theta_{1}\right)\right)-\kappa \text { on } F \cap\left\{\theta_{1} \leq \theta_{2}, \theta<\rho\right\}, \\
Y\left(\theta_{2}\right)>\tilde{\varphi}\left(\theta_{2}, X\left(\theta_{2}\right)\right)-\kappa \text { on } F \cap\left\{\theta_{1}>\theta_{2}, \theta<\rho\right\}, \\
Y(\rho)>\tilde{\varphi}(\rho, X(\rho))-\kappa \text { on } F \cap\{\theta \geq \rho\} .
\end{gathered}
$$

Since $\left(\theta_{1}, X\left(\theta_{1}\right)\right) \notin B_{\varepsilon / 2}\left(t_{0}, x_{0}\right)$, it follows from the first two inequalities in (3.4.7) that

$$
Y\left(\theta_{1}\right)>\tilde{\varphi}\left(\theta_{1}, X\left(\theta_{1}\right)\right)-\kappa>w\left(\theta_{1}, X\left(\theta_{1}\right)\right) \text { on } F \cap\left\{\theta_{1} \leq \theta_{2}, \theta<\rho\right\}
$$

On the other hand, since $Y\left(\theta_{2}\right)>\tilde{\varphi}\left(\theta_{2}, X\left(\theta_{2}\right)\right)-\kappa$ on $F \cap\left\{\theta_{1}>\theta_{2}, \theta<\rho\right\}$ and (3.4.8) holds,

$$
Y\left(\theta_{2}\right)-\tilde{\varphi}\left(\theta_{2}, X\left(\theta_{2}\right)\right) \geq \varepsilon \text { on } F \cap\left\{\theta_{1}>\theta_{2}, \theta<\rho\right\}
$$

Observing that $\left(\theta_{2}, X\left(\theta_{2}\right)\right) \in B_{\varepsilon / 2}\left(t_{0}, x_{0}\right)$ on $\left\{\theta_{1}>\theta_{2}\right\}$, we get from the last inequality of (3.4.7) that

$$
Y\left(\theta_{2}\right)>\tilde{\varphi}\left(\theta_{2}, X\left(\theta_{2}\right)\right)+\varepsilon>w\left(\theta_{2}, X\left(\theta_{2}\right)\right) \text { on } F \cap\left\{\theta_{1}>\theta_{2}, \theta<\rho\right\}
$$

From (3.4.11) and (3.4.12), we get that $Y(\theta)>w(\theta, X(\theta))$ on $F \cap\{\theta<\rho\}$. Therefore, from the definition of $\mathbb{U}_{\text {co }}^{+}$,

$$
\mathbb{P}\left(G_{0} \mid F \cap\{\theta<\rho\}\right)>0 \text { if } \mathbb{P}(F \cap\{\theta<\rho\})>0 .
$$

From (3.4.10), it holds that

$$
\mathbb{P}(G \mid F \cap\{\theta \geq \rho\})>0 \text { if } \mathbb{P}(F \cap\{\theta \geq \rho\})>0 .
$$

Since $G_{0} \subset G$, (3.4.13) and (3.4.14) imply that $\mathbb{P}(G \cap F)>0$. Therefore,

$$
\mathbb{P}\left(G \cap B \cap E_{1}\right)>0 .
$$




\section{Step 2 ( $u_{\text {co }}^{-}$is a viscosity super-solution).}

Step A: We show in this step that $u_{\text {co }}^{-}(t, x) \geq g(x)$ for all $(t, x) \in \mathbb{D}$. Assume, on the contrary, that for some $\left(t_{0}, x_{0}\right) \in \mathbb{D}$, there exists $\eta>0$ such that

$$
2 \eta=g\left(x_{0}\right)-u_{\mathrm{co}}^{-}\left(t_{0}, x_{0}\right)>0 .
$$

Choose an arbitrary $w \in \mathbb{U}_{\text {co }}^{-}$. By the definition of $\mathbb{U}_{\text {co }}^{-}$and lower semi-continuity of $g$, there exists $\varepsilon>0$ such that for all $(t, x) \in \bar{B}_{\varepsilon}\left(t_{0}, x_{0}\right)$,

$$
g(x)-w(t, x)>\eta, g(x)-g\left(x_{0}\right)>-\frac{\eta}{2},\left|w(t, x)-w\left(t_{0}, x_{0}\right)\right| \leq \frac{\eta}{2} .
$$

Define

$$
w^{\prime}(t, x):=\left\{\begin{array}{l}
w(t, x) \text { for }(t, x) \notin \bar{B}_{\varepsilon}\left(t_{0}, x_{0}\right), \\
w(t, x)+\left(g\left(x_{0}\right)-\eta-w\left(t_{0, x_{0}}\right)\right)\left(1-\frac{\operatorname{dist}\left((t, x),\left(t_{0}, x_{0}\right)\right)}{\varepsilon}\right), \text { otherwise. }
\end{array}\right.
$$

Obviously, $w^{\prime} \geq w$ and $w^{\prime}$ is continuous with polynomial growth. In addition,

$$
\begin{gathered}
\left\{(t, x): w(t, x)<w^{\prime}(t, x)\right\}=B_{\varepsilon}\left(t_{0}, x_{0}\right) \text { and } \\
\left.w^{\prime}(t, x) \leq w(t, x)+\left(g\left(x_{0}\right)-\eta-w\left(t_{0, x_{0}}\right)\right)<g(x) \text { for }(t, x) \in \bar{B}_{\varepsilon}\left(t_{0}, x_{0}\right)\right) .
\end{gathered}
$$

The equation above, along with the fact that $w \in \mathbb{U}_{\text {co }}^{-}$, implies that $w^{\prime}(T, x) \leq g(x)$ for all $x \in \mathbb{R}^{d}$. Noting that $w^{\prime}\left(t_{0}, x_{0}\right)=g\left(x_{0}\right)-\eta>u_{\text {co }}^{-}\left(t_{0}, x_{0}\right)$ due to (3.4.15), we would obtain a contradiction if we could show $w^{\prime} \in \mathbb{U}_{\text {co }}^{-}$. We now prove that $w^{\prime} \in \mathbb{U}_{\text {co }}^{-}$.

$\operatorname{Fix}(t, x, y) \in \mathbb{D}_{i} \times \mathbb{R}, \tau \in \mathcal{T}_{t}$ and $\nu \in \mathcal{U}_{\text {co }}^{t}$. For $w \in \mathbb{U}_{\text {co }}^{-}$, let $\rho^{w, \tau, \nu} \in \mathcal{T}_{\tau}$ and $\tilde{\nu}^{w, \tau, \nu}$ be the "optimal" stopping time and control satisfying the second item in Definition 3.4.2. In order to show that $w^{\prime} \in \mathbb{U}_{\text {co }}^{-}$, we want to construct an "optimal" stopping time $\rho$ and "optimal" control $\tilde{\nu}$ which work for $w^{\prime}$ in the sense of Definition 3.4.2. Let $A=\left\{w(\tau, X(\tau))=w^{\prime}(\tau, X(\tau))\right\} \in \mathcal{F}_{\tau}^{t}$,

$$
\rho=\mathbb{1}_{A} \rho^{w, \tau, \nu}+\mathbb{1}_{A^{c}} \tau \text { and } \tilde{\nu}=\left(\mathbb{1}_{A} \tilde{\nu}^{w, \tau, \nu}+\mathbb{1}_{A^{c}} u_{0}\right) \mathbb{1}_{[\tau, T]},
$$


where $u_{0}$ is an arbitrary element in $U$. Obviously, $\rho \in \mathcal{T}_{\tau}$ and $\tilde{\nu} \in \mathcal{T}_{t}$. It suffices to show

$$
Y(\rho) \leq g(X(\rho)) \mathbb{P} \text {-a.s. on }\left\{Y \leq w^{\prime}(\tau, X(\tau))\right\}
$$

(i) On $A \cap\left\{Y \leq w^{\prime}(\tau, X(\tau))\right\}$ : Note that $A \cap\left\{Y \leq w^{\prime}(\tau, X(\tau))\right\} \subset\{Y(\tau)<$ $w(\tau, X(\tau))\}$. From the fact $w \in \mathbb{U}_{\text {co }}^{-}$and the definition of $\rho$ and $\tilde{\nu}$ on $A$, it holds that

$$
Y(\rho)=Y\left(\rho^{w, \nu, \tau}\right) \leq g\left(X\left(\rho^{w, \nu, \tau}\right)\right)=g(X(\rho)) \text { on } A \cap\left\{Y \leq w^{\prime}(\tau, X(\tau))\right\}
$$

(ii) On $A^{c} \cap\left\{Y \leq w^{\prime}(\tau, X(\tau))\right\}:(\tau, X(\tau)) \in B_{\varepsilon}\left(t_{0}, x_{0}\right)$ on $A^{c}$ from (3.4.16), which implies $w^{\prime}(\tau, X(\tau))<g(X(\tau))$ from (3.4.17). This, together with the fact that $\rho=\tau$ on $A^{c}$, implies that

$$
Y(\rho) \leq w^{\prime}(\rho, X(\rho)) \leq g(X(\rho)) \text { on } A^{c} \cap\left\{Y \leq w^{\prime}(\tau, X(\tau))\right\}
$$

Step B: Assume, on the contrary, that for some $\left(t_{0}, x_{0}\right) \in \mathbb{D}_{i}$ and $\varphi \in C^{1,2}(\mathbb{D})$ satisfying $0=\left(u^{-}-\varphi\right)\left(t_{0}, x_{0}\right)=\min _{\mathbb{D}_{i}}\left(u_{\mathrm{co}}^{-}-\varphi\right)$, we have

$$
-4 \eta:=-\partial_{t} \varphi\left(t_{0}, x_{0}\right)+F^{*} \varphi\left(t_{0}, x_{0}\right)<0 .
$$

From Lemma 3.4.6, there exists a non-decreasing sequence $\mathbb{U}_{\mathrm{co}}^{-} \ni w_{k} \nearrow u_{\mathrm{co}}^{-}$. Fix such a sequence $\left\{w_{k}\right\}_{k=1}^{\infty}$ and an arbitrary stochastic sub-solution $w_{-}$. Let $\tilde{\varphi}(t, x)=$ $\varphi(t, x)-\iota\left|x-x_{0}\right|^{n_{0}}$. We can choose $n_{0} \geq 2$ such that for any $\iota>0$,

$$
\max _{0 \leq t \leq T}\left(\tilde{\varphi}(t, x)-w_{1}(t, x)\right) \rightarrow-\infty \text { as }|x| \rightarrow \infty
$$

We can do this because $\varphi(t, x)$ is bounded from above by $w_{-}$(which has polynomial growth in $x)$ and $w_{1}$ has polynomial growth in $x$. Since $\left(\mathcal{M}_{\varepsilon, \eta}\right)_{\varepsilon \geq 0}$ is non-increasing in $\varepsilon$, we know

$$
F_{*}(\Theta, \varphi)=\lim _{\substack{\Theta^{\prime} \rightarrow \Theta, \psi \\ \eta \searrow 0}} F_{0, \eta}\left(\Theta^{\prime}, \psi\right) .
$$


By (3.2.7) and (3.4.20), we can find $\varepsilon>0, \eta>0$ and $\iota>0$ such that for all $(t, x, y)$ satisfying $(t, x) \in B_{\varepsilon}\left(t_{0}, x_{0}\right)$ and $|y-\tilde{\varphi}(t, x)| \leq \varepsilon, \mu_{Y}(t, x, y, u)-\mathcal{L}^{u} \tilde{\varphi}(t, x) \leq$ $-2 \eta$ for some $u \in \mathcal{M}_{0,-\eta}(t, x, y, D \tilde{\varphi}(t, x), \tilde{\varphi})$. Fix $\iota$. Note that $\left(t_{0}, x_{0}\right)$ is still a strict minimizer of $u_{\text {co }}^{-}-\tilde{\varphi}$ over $\mathbb{D}_{i}$. For $\varepsilon$ sufficiently small, Assumption 3.4 .4 implies that there exists a locally Lipschitz map $\hat{\nu}$ such that

$$
\begin{gathered}
\qquad \hat{\nu}(t, x, y, D \tilde{\varphi}(t, x)) \in \mathcal{M}_{0,-\eta}(t, x, y, D \tilde{\varphi}(t, x), \tilde{\varphi}) \text { and } \\
\qquad \mu_{Y}(t, x, y, \hat{\nu}(t, x, y, D \tilde{\varphi}(t, x)))-\mathcal{L}^{\hat{\nu}(t, x, y, D \tilde{\varphi}(t, x))} \tilde{\varphi}(t, x) \leq-\eta \\
\text { for all }(t, x, y) \in \mathbb{D}_{i} \times \mathbb{R} \text { s.t. }(t, x) \in B_{\varepsilon}\left(t_{0}, x_{0}\right) \text { and }|y-\tilde{\varphi}(t, x)| \leq \varepsilon .
\end{gathered}
$$

In the arguments above, choose $\varepsilon$ small enough such that $\left.\bar{B}_{\varepsilon}\left(t_{0}, x_{0}\right)\right) \cap \mathbb{D}_{T}=\emptyset$. Since (3.4.21) holds, there exists $R_{0}>\varepsilon$ such that $\tilde{\varphi}<w_{1}-\varepsilon \leq w_{k}-\varepsilon$ on $\mathbb{O}:=$ $\mathbb{D}-[0, T] \times \bar{B}_{R_{0}}\left(x_{0}\right)$ for all $k$. On the compact set $\mathbb{T}:=[0, T] \times \bar{B}_{R_{0}}\left(x_{0}\right)-B_{\varepsilon / 2}\left(t_{0}, x_{0}\right)$, we know that $\tilde{\varphi}<u_{\mathrm{co}}^{-}$and the maximum of $\tilde{\varphi}-u_{\mathrm{co}}^{-}$is attained since $u_{\mathrm{co}}^{-}$is LSC. Therefore, $\tilde{\varphi}<u_{\text {co }}^{-}-2 \alpha$ on $\mathbb{T}$ for some $\alpha>0$. By a Dini-type argument, for large enough $n$, we have $\tilde{\varphi}<w_{n}-\alpha$ on $\mathbb{T}$ and $\tilde{\varphi}<w_{n}+\varepsilon$ on $\bar{B}_{\varepsilon / 2}\left(t_{0}, x_{0}\right)$. For simplicity, fix such an $n$ and set $w=w_{n}$. In short,

$$
\tilde{\varphi}<w-\varepsilon \text { on } \mathbb{O}, \tilde{\varphi}<w-\alpha \text { on } \mathbb{T} \text { and } \tilde{\varphi}<w+\varepsilon \text { on } \bar{B}_{\varepsilon / 2}\left(t_{0}, x_{0}\right) \text {. }
$$

For $\kappa \in(0, \varepsilon \wedge \alpha)$, define

$$
w^{\kappa}:=\left\{\begin{array}{l}
\left.(\tilde{\varphi}+\kappa) \vee w \text { on } \bar{B}_{\varepsilon}\left(t_{0}, x_{0}\right)\right) \\
w \text { outside } \bar{B}_{\varepsilon}\left(t_{0}, x_{0}\right)
\end{array}\right.
$$

Observing that $w^{\kappa}\left(t_{0}, x_{0}\right)=\tilde{\varphi}\left(t_{0}, x_{0}\right)+\kappa>u_{\mathrm{co}}^{-}\left(t_{0}, x_{0}\right)$, we could obtain a contradiction if we could show that $w^{\kappa} \in \mathbb{U}_{\mathrm{co}}^{-}$. Obviously, $w^{\kappa}$ is continuous, has polynomial growth in $x$ and $w^{\kappa}(T, x) \geq g(x)$ for all $x \in \mathbb{R}^{d}$.

Fix $(t, x, y) \in \mathbb{D}_{i} \times \mathbb{R}, \nu \in \mathcal{U}_{\text {co }}^{t}$ and $\tau \in \mathcal{T}_{t}$. Now our goal is to construct $\widetilde{\nu} \in \mathbb{U}_{\text {co }}^{t}$ 
and $\rho \in \mathcal{T}_{t}$ such that $w^{\kappa}$ and the processes $(X, Y)$ controlled by $\nu \otimes_{\tau} \widetilde{\nu}$ satisfy the property in Definition 3.4.2.

Let $A=\left\{w^{\kappa}\left(\tau, X_{t, x}^{\nu}(\tau)\right)=w\left(\tau, X_{t, x}^{\nu}(\tau)\right)\right\}$. On $A$, let $\widetilde{\nu}$ and $\rho$ be $\widetilde{\nu}_{1}$ and $\rho_{1}$, respectively, which are "optimal" for $w$ starting at $\tau$. We get the existence of $\widetilde{\nu}_{1}$ and $\rho_{1}$ since $w \in \mathbb{U}_{\mathrm{co}}^{-}$. On $A^{c}$, by an argument similar to that in [6] (see Step 1.1 of Theorem 3.1's proof), we can construct $\nu_{0} \in \mathcal{U}_{\text {co }}^{t}$ such that

$$
\begin{aligned}
\nu_{0}(s):= & \hat{\nu}\left(s, X_{t, x}^{\nu \otimes_{\tau} \nu_{0}}(s), Y_{t, x, y}^{\nu \otimes_{\tau} \nu_{0}}(s), D \tilde{\varphi}\left(s, X_{t, x}^{\nu \otimes_{\tau} \nu_{0}}(s)\right) \text { for } \tau \leq s<\theta:=\theta_{1} \wedge \theta_{2}\right. \\
& \text { where } \theta_{1}:=\inf \left\{s \in[\tau, T]:\left(s, X_{t, x}^{\nu \otimes_{\tau} \nu_{0}}(s)\right) \notin B_{\varepsilon / 2}\left(t_{0}, x_{0}\right)\right\} \wedge T, \\
& \theta_{2}:=\inf \left\{s \in[\tau, T]:\left|Y_{t, x, y}^{\nu \otimes_{\tau} \nu_{0}}(s)-\tilde{\varphi}\left(s, X_{t, x}^{\nu \otimes_{\tau} \nu_{0}}(s)\right)\right| \geq \varepsilon\right\} \wedge T .
\end{aligned}
$$

In the construction of $\nu_{0}$, we take advantage of Assumption 3.2.2 and the Lipschitz continuity of $\hat{\nu}$ which guarantee the existence of $X_{t, x}^{\nu \otimes_{\tau} \nu_{0}}$ and $Y_{t, x, y}^{\nu \otimes_{\tau} \nu_{0}}$. Since $X_{t, x}^{\nu \otimes_{\tau} \nu_{0}}$ and $Y_{t, x, y}^{\nu \otimes_{\tau} \nu_{0}}$ are càdlàg, it is easy to check that $\theta \in \mathcal{T}_{\tau}$. We also see that

$$
\begin{gathered}
\left(\theta_{1}, X_{t, x}^{\nu \otimes_{\tau} \nu_{0}}\left(\theta_{1}\right)\right) \notin B_{\varepsilon / 2}\left(t_{0}, x_{0}\right), \quad\left|Y_{t, x, y}^{\nu \otimes_{\tau} \nu_{0}}\left(\theta_{2}\right)-\tilde{\varphi}\left(\theta_{2}, X_{t, x}^{\nu \otimes_{\tau} \nu_{0}}\left(\theta_{2}\right)\right)\right| \geq \varepsilon \\
\left(\theta_{1}, X_{t, x}^{\nu \otimes_{\tau} \nu_{0}}\left(\theta_{1}-\right)\right) \in \bar{B}_{\varepsilon / 2}\left(t_{0}, x_{0}\right), \quad \mid Y_{t, x, y}^{\nu \otimes_{\tau} \nu_{0}}\left(\theta_{2}-\right)-\tilde{\varphi}\left(\theta_{2}, X_{t, x}^{\nu \otimes_{\tau} \nu_{0}}\left(\theta_{2}-\right) \mid \leq \varepsilon\right.
\end{gathered}
$$

Let $\widetilde{\nu}^{\theta}$ and $\rho^{\theta}$ be the "optimal" control and stopping time for $w$ given $\theta$ and the controlled processes $\left(X_{t, x}^{\nu \otimes_{\tau} \nu_{0}}, Y_{t, x}^{\nu \otimes_{\tau} \nu_{0}}\right)$. We define $\tilde{\nu}$ to be $\nu_{0}$ on $\left[\tau, \theta\left[\right.\right.$ and $\widetilde{\nu}^{\theta}$ after $\theta$ on the set $A^{c}$. We set $\rho$ to be $\rho^{\theta}$ on $A^{c}$. In short,

$$
\widetilde{\nu}:=\left(\mathbb{1}_{A} \widetilde{\nu}_{1}+\mathbb{1}_{A^{c}}\left(\nu_{0} \mathbb{1}_{[t, \theta)}+\mathbb{1}_{[\theta, T]} \widetilde{\nu}^{\theta}\right)\right) \mathbb{1}_{[\tau, T]} \text { and } \rho:=\mathbb{1}_{A} \rho_{1}+\mathbb{1}_{A^{c}} \rho^{\theta}
$$

It is not difficult to check that $\widetilde{\nu} \in \mathcal{U}_{\text {co }}^{t}$ and $\rho \in \mathcal{T}_{\tau}$. It suffices to show that

$$
Y(\rho) \leq g(X(\rho)) \mathbb{P}-\text { a.s. on } S:=\left\{Y(\tau) \leq w^{\kappa}(\tau, X(\tau))\right\}
$$

where $X:=X_{t, x}^{\nu \otimes_{\tau} \tilde{\nu}}$ and $Y:=Y_{t, x, y}^{\nu \otimes_{\tau} \tilde{\nu}}$. Corresponding to the construction of $\widetilde{\nu}$ on $A$ and $A^{c}$, we consider the following two cases: 
(i) On the set $A \cap S$. We have $Y(\tau) \leq w(\tau, X(\tau))$. From the definition of $\tilde{\nu}$ and $\rho$ on $A$ and the fact that $w \in \mathbb{U}_{\text {co }}^{-}$, we know

$$
Y(\rho)=Y_{t, x, y}^{\nu \otimes_{\tau} \widetilde{\nu}_{1}}\left(\rho_{1}\right) \leq g\left(X_{t, x}^{\nu \otimes_{\tau} \widetilde{\nu}_{1}}\left(\rho_{1}\right)\right) \leq g(X(\rho)) \mathbb{P}-\text { a.s on } A \cap S .
$$

(ii) On the set $A^{c} \cap S$. Letting $\Gamma(s):=Y(s)-\tilde{\varphi}(s, X(s))$, we use Itô's formula and the definition of $\nu_{0}$ to obtain

$$
\begin{aligned}
\Gamma(\cdot \wedge \theta) & =\Gamma(\tau)+\int_{\tau}^{\wedge} \int_{E} \bar{J}^{\nu_{0}(s), e}(s, Z(s-), \tilde{\varphi})^{\top} \lambda(d s, d e) \\
& +\int_{\tau}^{\cdot \wedge \theta}\left(\mu_{Y}\left(s, Z(s), \nu_{0}(s)\right)-\mathscr{L}^{\nu_{0}(s)} \tilde{\varphi}(s, X(s))\right) d s
\end{aligned}
$$

on $A \cap S$. Therefore, by (3.4.22), (3.4.23), (3.4.26) and the definition of $\theta$, we know that $\Gamma(\cdot \wedge \theta)$ is non-increasing on $[\tau, T]$. This implies that

$$
Y(\theta)-\tilde{\varphi}(\theta, X(\theta))-\kappa \leq Y(\tau)-\tilde{\varphi}(\tau, X(\tau))-\kappa \leq 0 \text { on } A^{c} \cap S .
$$

Since $\left(\theta_{1}, X\left(\theta_{1}\right)\right) \notin B_{\varepsilon / 2}\left(t_{0}, x_{0}\right)$, we know

$$
0 \geq Y\left(\theta_{1}\right)-\tilde{\varphi}\left(\theta_{1}, X\left(\theta_{1}\right)\right)-\kappa \geq Y\left(\theta_{1}\right)-w\left(\theta_{1}, X\left(\theta_{1}\right)\right) \text { on }\left\{\theta_{1} \leq \theta_{2}\right\} \cap A^{c} \cap S
$$

from (3.4.24). On the other hand, due to (3.4.25) and (3.4.27), it holds that

$$
Y\left(\theta_{2}\right)-\tilde{\varphi}\left(\theta_{2}, X\left(\theta_{2}\right)\right) \leq \varepsilon \text { on }\left\{\theta_{1}>\theta_{2}\right\} \cap A^{c} \cap S .
$$

Therefore, since $\tilde{\varphi}<w+\varepsilon$ on $\bar{B}_{\varepsilon / 2}\left(t_{0}, x_{0}\right)$ and (3.4.26) holds,

$$
Y\left(\theta_{2}\right) \leq \varepsilon+\tilde{\varphi}\left(\theta_{2}, X\left(\theta_{2}\right)\right)<w\left(\theta_{2}, X\left(\theta_{2}\right)\right) \text { on }\left\{\theta_{1}>\theta_{2}\right\} \cap A^{c} \cap S \text {. }
$$

Combining (3.4.28) and (3.4.29), we obtain $Y(\theta)-w(\theta, X(\theta)) \leq 0$ on $A^{c} \cap S$. Therefore, from the definitions of $\widetilde{\nu}^{\theta}$ and $\rho^{\theta}$,

$$
Y(\rho) \leq g(X(\rho)) \text { on } A^{c} \cap\left\{Y(\tau) \leq w^{\kappa}(\tau, X(\tau))\right\} .
$$




\subsubsection{Boundary conditions}

We then discuss the boundary conditions at terminal time $T$. From the definition

of the value function $u_{\mathrm{co}}$, it holds that $u_{\mathrm{co}}(T, x)=g(x)$ for all $x \in \mathbb{R}^{d}$. However, $u_{\mathrm{co}}^{+}$ and $u_{\mathrm{co}}^{-}$may not agree with this boundary condition. Let

$$
u_{\mathrm{co}}^{+}(T-, x)=\limsup _{\left(t<T, x^{\prime}\right) \rightarrow(T, x)} u_{\mathrm{co}}^{-}\left(t, x^{\prime}\right), \quad u_{\mathrm{co}}^{-}(T-, x)=\liminf _{\left(t<T, x^{\prime}\right) \rightarrow(T, x)} u_{\mathrm{co}}^{-}\left(t, x^{\prime}\right) .
$$

Theorem 3.4.8. Under Assumptions 3.2.1, 3.2.2, 3.4.3 and 3.4.4, $u_{c o}^{+}(T-, \cdot)$ is an USC viscosity sub-solution of

$$
(\varphi(x)-g(x)) \mathbb{1}_{\left\{F_{*} \varphi(x)>-\infty\right\}} \leq 0 \text { on } \mathbb{R}^{d}
$$

and

$$
u_{c o}^{-}(T-, x) \geq g(x) \text { for all } x \in \mathbb{R}^{d} .
$$

Proof. Step 1.(The sub-solution property on $\mathbb{D}_{T}$ ) We show in this step that $u_{\mathrm{co}}^{+}(T-, \cdot)$ is a viscosity sub-solution of

$$
(\varphi(x)-g(x)) \mathbb{1}_{\left\{F_{*} \varphi(x)>-\infty\right\}} \leq 0
$$

Let $x_{0} \in \mathbb{R}^{d}$ and $\varphi \in C^{2}\left(\mathbb{R}^{d}\right)$ be a smooth function such that

$$
0=\left(u_{b}^{+}\left(T-, x_{0}\right)-\varphi\left(x_{0}\right)\right)=\max _{x \in \mathbb{R}^{d}}(\text { strict })\left(u_{\mathrm{co}}^{+}(T-, x)-\varphi(x)\right) .
$$

Assume that $F_{*} \varphi\left(x_{0}\right)=C>-\infty$ and $g\left(x_{0}\right)<u_{\mathrm{co}}^{+}\left(T-, x_{0}\right)=\varphi\left(x_{0}\right)$ and we will work towards a contradiction. Assume without loss of generality that

$$
\varphi(x) \rightarrow \infty \text { as }|x| \rightarrow \infty
$$

Let

$$
\tilde{\varphi}^{\prime}(x)=\varphi(x)+\iota\left|x-x_{0}\right|^{4}, \quad \tilde{\varphi}(t, x)=\varphi(x)+\iota\left|x-x_{0}\right|^{4}+(C+2)(T-t),
$$


where $\iota$ will be fixed later. Note that $D \tilde{\varphi}^{\prime}(x)=D \tilde{\varphi}(t, x), D^{2} \tilde{\varphi}^{\prime}(x)=D^{2} \tilde{\varphi}(t, x)$. By the facts that $g\left(x_{0}\right)<\varphi\left(x_{0}\right)=\tilde{\varphi}\left(T, x_{0}\right)=u_{\mathrm{co}}^{+}\left(T-, x_{0}\right)$, upper semi-continuity of $g$ and $u_{\mathrm{co}}^{+}$and the regularity of $\varphi$, the locally boundedness of $\mu_{X}, \sigma_{X}, \mu_{Y}, b$ and $\beta$, the regularity of $\varphi$ and definition of $F_{*}$, there exist $\iota, \varepsilon>0$ and $0<\eta<1$ such that there exist $\iota, \varepsilon>0$ and $0<\eta<1$ such that

$$
\begin{gathered}
\tilde{\varphi}(t, x)-g(x)>\varepsilon \text { for }(t, x) \in \bar{B}_{\varepsilon}\left(T, x_{0}\right), \\
\tilde{\varphi}>u_{\mathrm{co}}^{+}-2 \eta \text { on }[T-\varepsilon, T) \times \bar{B}_{\varepsilon / 2}\left(x_{0}\right),
\end{gathered}
$$

and

$$
\mu_{Y}(t, x, y, u)-\mu_{X}^{\top}(t, x, u) D \tilde{\varphi}(t, x)-\frac{1}{2} \operatorname{Tr}\left[\sigma_{X} \sigma_{X}^{\top}(t, x, u) D^{2} \tilde{\varphi}(t, x)\right] \geq C-1
$$

for all $(t, x, y, u) \in \mathbb{D} \times \mathbb{R} \times U$ s.t. $(t, x) \in[T-\varepsilon, T] \times \bar{B}_{\varepsilon}\left(x_{0}\right),|y-\tilde{\varphi}(t, x)| \leq \varepsilon$ and $u \in \mathcal{M}_{\varepsilon, \eta}\left(t, x, y, D \tilde{\varphi}(t, x), \tilde{\varphi}^{\prime}\right)$.

Then by the definition of $\Pi^{u, e}$ involved in $\mathcal{M}_{\varepsilon, \eta}$,

$$
\begin{aligned}
& \mu_{Y}(t, x, y, u)-\mathscr{L}^{u} \tilde{\varphi}(t, x) \geq C-1+C+2 \geq \eta \text { for }(t, x, y) \in \mathbb{D} \times \mathbb{R} \times U \text { s.t. } \\
& (t, x) \in[T-\varepsilon, T] \times \bar{B}_{\varepsilon}\left(x_{0}\right),|y-\tilde{\varphi}(t, x)| \leq \varepsilon \text { and } u \in \mathcal{M}_{\varepsilon, \eta}(t, x, y, D \tilde{\varphi}(t, x), \tilde{\varphi}) .
\end{aligned}
$$

From Lemma 3.4.6, there exists a non-decreasing sequence $\mathbb{U}_{b}^{+} \ni w_{n} \searrow u_{\mathrm{co}}^{+}$. Fix this sequence. Then by a Dini type argument, from (3.4.31), there exists an $n_{0}$ such that

$$
\tilde{\varphi}>w_{n_{0}}-\eta \text { on }[T-\varepsilon, T) \times \bar{B}_{\varepsilon / 2}\left(x_{0}\right) .
$$

By (3.4.30), there exists some $R_{0}>\varepsilon$ such that for $n \geq n_{0}$,

$$
\tilde{\varphi}(t, x)>w_{n_{0}}(t, x)+\varepsilon \geq w_{n}(t, x)+\varepsilon \text { on } \mathbb{O}:=[T-\varepsilon, T] \times\left(\mathbb{R}^{d}-\bar{B}_{R_{0}}\left(x_{0}\right)\right) .
$$

Since $\tilde{\varphi}(T, x) \geq \varphi(x)$, then $u_{\mathrm{co}}^{+}(T-, \cdot)-\tilde{\varphi}(T, \cdot)$ is strictly negative on the compact set $\mathbb{T}^{*}:=\bar{B}_{R_{0}}\left(x_{0}\right)-B_{\varepsilon / 2}\left(x_{0}\right)$. Hence, by the upper semi-continuity of $u_{\mathrm{co}}^{+}$, there exists some $\alpha>0$ such that

$$
\tilde{\varphi}(T, \cdot)>u_{\mathrm{co}}^{+}(T-, \cdot)+4 \alpha \text { on } \mathbb{T}^{*} .
$$


Similar to the Step 1 in this proof, there exists a $\sigma \leq \varepsilon$ and $n_{1} \geq n_{0}$ such that

$$
\tilde{\varphi}>w_{n_{1}}+\alpha \text { on }[T-\sigma, T) \times \mathbb{T}^{*}
$$

Let $w=w_{n_{1}}$. Define, for $\kappa \in(0, \varepsilon \wedge \delta \wedge \alpha)$,

$$
w^{\kappa} \triangleq\left\{\begin{array}{l}
(\tilde{\varphi}-\kappa) \wedge w \text { on }[T-\sigma, T] \times \bar{B}_{\varepsilon}\left(x_{0}\right), \\
w \text { outside }[T-\sigma, T] \times \bar{B}_{\varepsilon}\left(x_{0}\right) .
\end{array}\right.
$$

Similarly as Step 1 in the proof of Theorem 3.4.7, we can show that $w^{\kappa} \in \mathbb{U}_{\text {co }}^{+}$, which yields a contradiction.

Step 2. (The super-solution property on $\mathbb{D}_{T}$ ) We've already proved that $u_{\mathrm{co}}^{-}(t, x) \geq g(x)$ in Step $2 \mathrm{~A}$ of Theorem 3.4.7, which implies that $u_{\mathrm{co}}^{-}(T-, x) \geq$ $g(x)$

\subsubsection{Verification by comparison}

We now carry out the verification for non-smooth functions assuming the comparison principle.

Assumption 3.4.9. Let $F=F^{*}$. Assume that $F=F_{*}$ on the set $\{F>-\infty\}$ and that there exists a USC function $G: \mathbb{D} \times \mathbb{R} \times \mathbb{R}^{d} \times \mathbb{M}^{d} \times C(\mathbb{D}) \rightarrow \mathbb{R}$ such that

$$
\begin{aligned}
& \text { (a) } F(t, x, y, p, A, \varphi)>-\infty \Longrightarrow G(t, x, y, p, A, \varphi) \geq 0, \\
& \text { (b) } G(t, x, y, p, A, \varphi)>0 \Longrightarrow F(t, x, y, p, A, \varphi)>-\infty .
\end{aligned}
$$

Proposition 3.4.10. Under Assumptions Assumptions 3.2.1, 3.2.2, 3.4.3, 3.4.4 and 3.4.9, $u_{c o}^{+}$(resp. $\left.u_{c o}^{-}\right)$is a USC (resp. an LSC) viscosity sub-solution (resp. supersolution) of

$$
\min \left\{\varphi(t, x)-g(x), \max \left\{-\partial_{t} \varphi(t, x)+F \varphi(t, x), G \varphi(t, x)\right\}\right\}=0 \text { on } \mathbb{D}_{i}
$$

Moverover, $u_{c o}^{+}(T-, \cdot)$ (resp. $\left.u_{c o}^{-}(T-, \cdot)\right)$ is a USC (resp. an LSC) viscosity subsolution (resp. super-solution) of $\max \{\varphi(x)-g(x), G(x)\}=0$. 
Assumption 3.4.11. Assume that a comparison principle holds between USC subsolutions and LSC super-solutions for

$$
\min \{\varphi(x)-g(x), G \varphi(x)\}=0 \text { on } \mathbb{R}^{d} .
$$

Lemma 3.4.12. Under Assumptions 3.2.1, 3.2.2, 3.4.3, 3.4.4, 3.4.9 and 3.4.11, $u_{c o}^{-}(T-, \cdot)=u_{c o}^{+}(T-, \cdot)=\hat{g}(\cdot)$, where $\hat{g}$ is the unique continuous viscosity solution to $(3.4 .35)$.

Proof. The proof of this lemma follows same argument as in Lemma 3.3.12

Theorem 3.4.13. Suppose that there is a comparison principle for

$$
\min \left\{\varphi(t, x)-g(x), \max \left\{-\partial_{t} \varphi(t, x)+F \varphi(t, x), G \varphi(t, x)\right\}\right\}=0 \text { on } \mathbb{D}_{i}
$$

and that Assumptions 3.2.1, 3.2.2, 3.4.3, 3.4.4, 3.4.9 and 3.4.11 hold. Then there exists a unique continuous viscosity solution $V_{c o}$ to (3.4.36) with terminal condition $V_{c o}(T, \cdot)=\hat{g}(\cdot)$ and $u_{c o}(t, x)=u_{c o}^{-}(t, x)=u_{c o}^{+}(t, x)=V_{c o}(t, x)$ for $(t, x) \in \mathbb{D}_{i}$.

Proof. Define

$$
\hat{u}_{\mathrm{unco}}^{+}(t, x):= \begin{cases}u_{\mathrm{co}}^{+}(t, x), & (t, x) \in \mathbb{D}_{i} \\ \hat{g}(x), & t=T, x \in \mathbb{R}^{d}\end{cases}
$$

and

$$
\hat{u}_{\mathrm{co}}^{-}(t, x):= \begin{cases}u_{\mathrm{co}}^{-}(t, x), & (t, x) \in \mathbb{D}_{i}, \\ \hat{g}(x), & t=T, x \in \mathbb{R}^{d} .\end{cases}
$$

From Proposition 3.4.10, $\hat{u}_{\mathrm{co}}^{-}$is an LSC viscosity super-solution and $\hat{u}_{\mathrm{co}}^{+}$is a USC viscosity sub-solution of $(3.4 .36)$. Since $\hat{u}_{\mathrm{co}}^{+}(T, \cdot)=\hat{u}_{\mathrm{co}}^{-}(T, \cdot), \hat{u}_{\mathrm{co}}^{+} \leq \hat{u}_{\mathrm{co}}^{-}$on $\mathbb{D}$ by comparison. Hence, $\hat{u}_{\mathrm{co}}^{+}=\hat{u}_{\mathrm{co}}^{-}$on $\mathbb{D}$ from (3.4.1). Define $V_{\mathrm{co}}:=\hat{u}_{\mathrm{co}}^{+}=\hat{u}_{\mathrm{co}}^{-}$. It is a continuous viscosity solution of (3.4.36) satisfying $V_{\text {co }}(T, x)=\hat{g}(x)$. Uniqueness follows directly from the comparison principle. 


\subsubsection{Stochastic cooperative controller-stopper problem as a stochastic target prob- lem with a cooperative stopper}

In this subsection, we show that a stochastic cooperative controller-stopper problem can be expressed in terms of a stochastic target problem with a cooperative stopper. Given a bounded continuous function $g: \mathbb{R}^{d} \rightarrow \mathbb{R}$, we define

$$
\mathbf{u}_{\mathrm{co}}(t, x):=\sup _{\nu \in \mathcal{U}^{t}} \sup _{\rho \in \mathcal{T}_{t}} \mathbb{E}\left[g\left(X_{t, x}^{\nu}(\rho)\right)\right]
$$

We follow the setup of Section 3.2 with one exception: $\mathcal{U}^{t}$ is the collection of all the $\mathbb{F}^{t}$-predictable processes in $\mathbb{L}^{2}\left(\Omega \times[0, T], \mathcal{F} \otimes \mathcal{B}[0, T], \mathbb{P} \otimes \lambda_{L} ; U\right)$, where $U \subset \mathbb{R}^{d}$ and $X$ follows the SDE

$d X(s)=\mu_{X}(s, X(s), \nu(s)) d s+\sigma_{X}(s, X(s), \nu(s)) d W_{s}+\int_{E} \beta(s, X(s-), \nu(s), e) \lambda(d s, d e)$.

To convert the cooperative controller-stopper problem to a stochastic target problem with a cooperative stopper, we now prove the following lemma. The proof relies on Lemma 3.5.7.

Lemma 3.4.14. Suppose Assumptions 3.2.1 and 3.2.2 hold. Define a stochastic target problem as follows:

$$
\begin{aligned}
u_{c o}(t, x):= & \sup \left\{y \in \mathbb{R}: \exists(\nu, \alpha, \gamma, \rho) \in \mathcal{U}^{t} \times \mathcal{A}^{t} \times \Gamma_{c o}^{t} \times \mathcal{T}_{t} \text { s.t. } Y_{t, y}^{\alpha, \gamma}(\rho) \leq g\left(X_{t, x}^{\nu}(\rho)\right)\right\} \\
& \text { where } Y_{t, y}^{\alpha, \gamma}(\cdot):=y+\int_{t} \alpha^{\top}(s) d W_{s}+\int_{t} \int_{E} \gamma^{\top}(s, e) \tilde{\lambda}(d s, d e) .
\end{aligned}
$$

Here, $\mathcal{A}^{t}$ and $\Gamma_{\text {co }}^{t}$ are the collections of $\mathbb{R}^{d}$-valued and $\mathbb{L}^{2}\left(E, \mathcal{E}, \hat{m} ; \mathbb{R}^{I}\right)$-valued processes, respectively, satisfying the admissibility conditions in Section 3.2. Then $u_{c o}=\mathbf{u}_{c o}$ on $\mathbb{D}$.

Proof. In view of Lemma 3.5.7 and Remark 3.5.8, it suffices to check that

$$
\mathcal{M}_{\mathrm{co}} \subset \mathcal{M}:=\left\{Y_{t, y}^{\alpha, \gamma}(\cdot): y \in \mathbb{R}, \alpha \in \mathcal{A}^{t}, \gamma \in \Gamma^{t}\right\}
$$


where $\mathcal{M}_{\text {co }}$ is defined as in Remark 3.5.8. In fact, by the martingale representation theorem, for any $\nu \in \mathcal{U}^{t}$ and $\rho \in \mathcal{T}_{t}, \mathbb{E}\left[g\left(X_{t, x}^{\nu}(\rho)\right) \mid \mathcal{F}^{t}\right]$ can be represented in the form of $Y_{t, y}^{\alpha, \gamma}$ for some $\alpha \in \mathcal{A}^{t}$ and $\gamma \in \Gamma_{0}^{t}$, where $\Gamma_{0}^{t}$ is the collection of $\mathbb{L}^{2}\left(E, \mathcal{E}, \hat{m} ; \mathbb{R}^{I}\right)$-valued processes satisfying all of the admissibility conditions except for (3.2.3). Assume, contrary to (3.4.37), that there exists $\nu_{0} \in \mathcal{U}^{t}$ and $\rho \in \mathcal{T}_{t}$ such that

$$
\mathbb{E}\left[g\left(X_{t, x}^{\nu_{0}}(\rho)\right) \mid \mathcal{F}^{t}\right]=y+\int_{t}^{\cdot} \alpha_{0}^{\top}(s) d W_{s}+\int_{t} \int_{E} \gamma_{0}^{\top}(s, e) \tilde{\lambda}(d s, d e)
$$

for some $y \in \mathbb{R}, \alpha_{0} \in \mathcal{A}^{t}$ and $\gamma_{0} \in \Gamma_{0}^{t}$, but (3.2.3) does not hold. In the equation above, $\mathbb{E}\left[g\left(X_{t, x}^{\nu_{0}}(\rho)\right) \mid \mathcal{F}^{t}\right]$ can be chosen to be càdlàg, thanks to Theorem 1.3.13 in [20]. Then for $K>2\|g\|_{\infty}$, there exists $\tau_{0} \in \mathcal{T}_{t}$ such that

$$
\mathbb{P}\left(\int_{E} \gamma_{0}^{\top}\left(\tau_{0}, e\right) \lambda\left(\left\{\tau_{0}\right\}, d e\right)>K\right)>0 .
$$

Let $M_{0}(\cdot)=\mathbb{E}\left[g\left(X_{t, x}^{\nu_{0}}(\rho)\right) \mid \mathcal{F}^{t}\right]$. Therefore,

$$
M_{0}\left(\tau_{0}\right)-M_{0}\left(\tau_{0}-\right)=\int_{E} \gamma_{0}^{\top}\left(\tau_{0}, e\right) \lambda\left(\left\{\tau_{0}\right\}, d e\right)>K \text { with positive probability }
$$

Since $\left|M_{0}\right|$ is bounded by $\|g\|_{\infty}<K / 2$, we obtain a contradiction.

Let $\mathbf{F}_{*}$ be the LSC envelope of the USC map $\mathbf{F}: \mathbb{D} \times \mathbb{R}^{d} \times \mathbb{M}^{d} \times C(\mathbb{D}) \rightarrow \mathbb{R}$ defined by

$$
\begin{gathered}
\mathbf{F}:(t, x, p, A, \varphi) \rightarrow \inf _{u \in U}\left\{-I[\varphi](t, x, u)-\mu_{X}^{\top}(t, x, u) p-\frac{1}{2} \operatorname{Tr}\left[\sigma_{X} \sigma_{X}^{\top}(t, x, u) A\right]\right\}, \\
\text { where } I[\varphi](t, x, u)=\sum_{1 \leq i \leq I} \int_{E}\left(\varphi\left(t, x+\beta_{i}(t, x, u, e)\right)-\varphi(t, x)\right) m_{i}(d e) .
\end{gathered}
$$

Theorem 3.4.15. Under Assumptions 3.2.1 and 3.2.2, $u_{c o}^{+}$is a USC viscosity subsolution of

$$
\min \left\{\varphi(t, x)-g(x),-\partial_{t} \varphi(t, x)+\mathbf{F}_{*} \varphi(t, x)\right\} \leq 0 \text { on } \mathbb{D}_{i}
$$

and $u_{c o}^{-}(T-, x) \leq g(x)$ for all $x \in \mathbb{R}^{d}$. On the other hand, $u_{c o}^{-}$is an LSC viscosity super-solution of

$$
\min \left\{\varphi(t, x)-g(x),-\partial_{t} \varphi(t, x)+\mathbf{F} \varphi(t, x)\right\} \geq 0 \text { on } \mathbb{D}_{i}
$$


and $u_{c o}^{-}(T-, \cdot) \geq g(x)$ for all $x \in \mathbb{R}^{d}$.

Proof. It is easy to check Assumption 3.4.4 for the stochastic target problem. Since $g$ is bounded, we can check that all of the assumptions in the appendix (Subsection 3.5.1) are satisfied, which implies that Assumption 3.3.3 holds. From Theorem 3.4.7, $u_{\mathrm{co}}^{+}$is a USC viscosity sub-solution of

$$
\min \left\{\varphi(t, x)-g(x),-\partial_{t} \varphi(t, x)+F_{*} \varphi(t, x)\right\} \leq 0 \text { on } \mathbb{D}_{i}
$$

and $u_{\mathrm{co}}^{-}$is an LSC viscosity super-solution of

$$
\min \left\{\varphi(t, x)-g(x),-\partial_{t} \varphi(t, x)+F^{*} \varphi(t, x)\right\} \geq 0 \text { on } \mathbb{D}_{i}
$$

From Proposition 3.1 in [13], $F^{*} \leq \mathbf{F}$ and $F_{*} \geq \mathbf{F}_{*}$. This implies that the viscosity properties in the parabolic interior and at the boundary hold.

The following two corollaries show that $\mathbf{u}_{\mathrm{co}}$ is the unique viscosity solution to its associated HJB equation. We omit the proofs.

Corollary 3.4.16. Suppose that Assumptions 3.2.1 and 3.2.2 hold, $\mathbf{F}=\mathbf{F}_{*}$ on $\{\mathbf{F}>-\infty\}$ and there exists a USC function $\mathbf{G}: \mathbb{D} \times \mathbb{R} \times \mathbb{R}^{d} \times \mathbb{M}^{d} \times C(\mathbb{D}) \rightarrow \mathbb{R}$ such that

(a) $\mathbf{F}(t, x, y, p, M, \varphi)>-\infty \Longrightarrow \mathbf{G}(t, x, y, p, M, \varphi) \geq 0$,

(b) $\mathbf{G}(t, x, y, p, M, \varphi)>0 \Longrightarrow \mathbf{F}(t, x, y, p, M, \varphi)>-\infty$.

Then $u_{c o}^{+}\left(\right.$resp. $\left.u_{c o}^{-}\right)$is a USC (resp. an LSC) viscosity sub-solution (resp. supersolution) of

$$
\min \left\{\varphi(t, x)-g(x), \max \left\{-\partial_{t} \varphi(t, x)+\mathbf{F} \varphi(t, x), \mathbf{G} \varphi(t, x)\right\}\right\}=0 \text { on } \mathbb{D}_{i}
$$

Corollary 3.4.17. Suppose that all the assumptions in Corollary 3.4.16 hold. Then $u_{c o}^{+}(T-, x)=u_{c o}^{-}(T-, x)=g(x)$. Moreover, if the comparison principle holds for

$$
\min \left\{\varphi(t, x)-g(x), \max \left\{-\partial_{t} \varphi(t, x)+\mathbf{F} \varphi(t, x), \mathbf{G} \varphi(t, x)\right\}\right\}=0 \text { on } \mathbb{D}_{i},
$$


then $u_{c o}\left(=\mathbf{u}_{c o}\right)$ is the unique continuous viscosity solution with $u_{c o}(T, x)=g(x)$.

\subsection{Appendix}

\subsubsection{The nonemptiness of $\mathbb{U}_{\text {unco }}^{+}, \mathbb{U}_{\text {unco }}^{-}, \mathbb{U}_{\text {co }}^{+}$and $\mathbb{U}_{\text {co }}^{-}$}

Assumption 3.5.1. $g$ is bounded.

Assumption 3.5.2. There exists $u_{0} \in U$ such that

$$
\sigma_{Y}\left(t, x, y, u_{0}\right)=0 \quad \text { and } b\left(t, x, y, u_{0}(e), e\right)=0
$$

for all $(t, x, y, e) \in \mathbb{D} \times \mathbb{R} \times E$.

Proposition 3.5.3. Under Assumptions 3.2.1, 3.2.2, 3.5.1 and 3.5.2, $\mathbb{U}_{\text {unco }}^{+}$and $\mathbb{U}_{c o}^{-}$ are not empty.

Proof. We will only show $\mathbb{U}_{\text {unco }}^{+}$is not empty. A very similar proof applies to $\mathbb{U}_{\text {co }}^{-}$.

Step 1. In this step we assume that $\mu_{Y}$ is non-decreasing in its $y$-variable. We will show that $w(t, x)=\gamma-e^{k t}$ is a stochastic super-solution for some choice of $k$ and $\gamma$.

By the linear growth condition on $\mu_{Y}$ in Assumption 3.2.2, there exists $L>0$ such that

$$
\left|\mu_{Y}\left(t, x, y, u_{0}\right)\right| \leq L(1+|y|)
$$

where $u_{0}$ is the element in $U$ in Assumption 3.5.2. Choose $k \geq 2 L$ and $\gamma$ such that $-e^{k T}+\gamma \geq\|g\|_{\infty}$. Then $w(t, x) \geq w(T, x) \geq g(x)$ for all $(t, x) \in \mathbb{D}$. It suffices to show that for any $(t, x, y) \in \mathbb{D} \times \mathbb{R}, \tau \in \mathcal{T}_{t}, \nu \in \mathcal{U}_{\text {unco }}^{t}$ and $\rho \in \mathcal{T}_{\tau}$,

$$
Y(\rho) \geq w(\rho, X(\rho)) \mathbb{P} \text {-a.s. on }\{Y(\tau) \geq w(\tau, X(\tau))\}
$$

where $X:=X_{t, x}^{\nu \otimes_{\tau} u_{0}}$ and $Y:=Y_{t, x, y}^{\nu \otimes_{\tau} u_{0}}$. Let $A=\{Y(\tau)>w(\tau, X(\tau))\}, V(s)=$ $w(s, X(s))$ and $\Gamma(s)=(V(s)-Y(s)) \mathbb{1}_{A}$. Therefore, for $s \geq \tau$,

$$
\Gamma(s)=\mathbb{1}_{A} \int_{\tau}^{s}(\xi(q)+\Delta(q)) d q, \text { where }
$$




$$
\begin{aligned}
\Delta(s): & =-k e^{k s}-\mu_{Y}\left(s, X(s), Y(s), u_{0}\right) \\
& \leq-k e^{k s}-\mu_{Y}\left(s, X(s),-e^{k s}, u_{0}\right) \\
& \leq-k e^{k s}+L\left(1+e^{k s}\right) \leq 0 \\
\xi(s): & =\mu_{Y}\left(s, X(s), V(s), u_{0}\right)-\mu_{Y}\left(s, X(s), Y(s), u_{0}\right) .
\end{aligned}
$$

Therefore, from (3.5.2) it holds that

$$
\Gamma(s) \leq \mathbb{1}_{A} \int_{\tau}^{s} \xi(q) d q \text { and } \Gamma^{+}(s) \leq \mathbb{1}_{A} \int_{\tau}^{s} \xi^{+}(q) d q \text { for } s \geq \tau
$$

From the Lipschitz continuity of $\mu_{Y}$ in $y$-variable in Assumption 3.2.2,

$$
\Gamma^{+}(s) \leq \mathbb{1}_{A} \int_{\tau}^{s} \xi^{+}(q) d q \leq \int_{\tau}^{s} L_{0} \Gamma^{+}(q) d q \text { for } s \geq \tau
$$

where $L_{0}$ is the Lipschitz constant of $\mu_{Y}$ with respect to $y$. Note that we use the assumption that $\mu_{Y}$ is non-decreasing in its $y$-variable to obtain the second inequality. Since $\Gamma^{+}(\tau)=0$, an application of Grönwall's Inequality implies that $\Gamma^{+}(\rho) \leq 0$, which further implies that (3.5.1) holds.

Step 2. We get rid of our assumption on $\mu_{Y}$ from Step 1 by following a proof similar to those in [16]. For $c>0$, define $\widetilde{Y}_{t, x, y}^{\nu}$ as the strong solution of

$$
\begin{aligned}
d \tilde{Y}(s) & =\tilde{\mu}_{Y}\left(s, X_{t, x}^{\nu}(s), \tilde{Y}(s), \nu(s)\right) d s+\tilde{\sigma}_{Y}^{\top}\left(s, X_{t, x}^{\nu}(s), \tilde{Y}(s), \nu(s)\right) d W_{s} \\
& +\int_{E} \tilde{b}^{\top}\left(s, X_{t, x}^{\nu}(s-), \tilde{Y}(s-), \nu_{1}(s), \nu_{2}(s, e), e\right) \lambda(d s, d e)
\end{aligned}
$$

with initial data $\widetilde{Y}(t)=y$, where

$$
\begin{gathered}
\widetilde{\mu}_{Y}(t, x, y, u):=c y+e^{c t} \mu_{Y}\left(t, x, e^{-c t} y, u\right), \widetilde{\sigma}_{Y}(t, x, y, u):=e^{c t} \sigma_{Y}\left(t, x, e^{-c t} y, u\right), \\
\widetilde{b}(t, x, y, u(e), e):=e^{c t} b\left(t, x, e^{-c t} y, u(e), e\right) .
\end{gathered}
$$

Therefore,

$$
\tilde{Y}_{t, x, y}^{\nu}(s) e^{-c s}=Y_{t, x, y e^{-c t}}^{\nu}(s), t \leq s \leq T \text {. }
$$


Let

$$
\tilde{u}_{\text {unco }}(t, x)=\inf \left\{y \in \mathbb{R}: \exists \nu \in \mathcal{U}_{\text {unco }}^{t} \text {, s.t. } \tilde{Y}_{t, x, y}^{\nu}(\rho) \geq \tilde{g}\left(\rho, X_{t, x}^{\nu}(\rho)\right) \text {-a.s. }\right\} \text {, }
$$

where $\tilde{g}(t, x)=e^{c t} g(x)$. Therefore, from (3.5.3), $\tilde{u}_{\text {unco }}(t, x)=e^{c t} u_{\text {unco }}(t, x)$. Since $\mu_{Y}$ is Lipschitz in $y$, we can choose $c>0$ so that

$$
\widetilde{\mu}_{Y}:(t, x, y, u) \mapsto c y+e^{c t} \mu_{Y}\left(t, x, e^{-c t} y, u\right)
$$

is non-decreasing in $y$. Moreover, all the properties of $\widetilde{\mu}_{Y}, \widetilde{\sigma}_{Y}$ and $\widetilde{b}$ in Assumption 3.2.2 still hold. We replace $\mu_{Y}, \sigma_{Y}$ and $b$ in all of the equations and definitions in Section 3.2 with $\widetilde{\mu}_{Y}, \widetilde{\sigma}_{Y}$ and $\widetilde{b}$, we get $\widetilde{H}^{*}$ and $\widetilde{H}_{*}$. Let $\widetilde{\mathbb{U}}_{\text {unco }}^{+}$be the set of stochastic super-solutions of the new target problem (3.5.4). It is easy to see that $w \in \mathbb{U}_{\text {unco }}^{+}$ if and only if $\widetilde{w}(t, x):=e^{c t} w(t, x) \in \widetilde{\mathbb{U}}_{\text {unco }}^{+}$. From Step 1, $\widetilde{\mathbb{U}}_{\text {unco }}^{+}$is not empty. Thus, $\mathbb{U}_{\text {unco }}^{+}$is not empty.

Assumption 3.5.4. There is $C \in \mathbb{R}$ such that for all $(t, x, y, u, e) \in \mathbb{D} \times \mathbb{R} \times U \times E$,

$$
\left|\mu_{Y}(t, x, y, u)+\int_{E} b^{\top}(t, x, y, u(e), e) m(d e)\right| \leq C(1+|y|) .
$$

Proposition 3.5.5. Under Assumptions 3.2.1, 3.2.2, 3.5.1 and 3.5.4, $\mathbb{U}_{\text {unco }}^{-}$and $\mathbb{U}_{c o}^{+}$ are not empty.

Proof. We will only show that $\mathbb{U}_{\text {unco }}^{-}$is not empty. Assume that

$$
\mu_{Y}(t, x, y, u)+\int_{E} b^{\top}(t, x, y, u(e), e) m(d e)
$$

is non-decreasing in its $y$-variable. We could remove this assumption by using the argument from previous proposition.

Choose $k \geq 2 C$ ( $C$ is the constant in Assumption 3.5.4) and $\gamma>0$ such that $e^{k T}-\gamma<-\|g\|_{\infty}$. Let $w(t, x)=e^{k x}-\gamma$. Notice that $w$ is continuous, has polynomial 
growth in $x$ and $w(T, x) \leq g(x)$ for all $x \in \mathbb{R}^{d}$. It suffices to show that for any $(t, x, y) \in \mathbb{D} \times \mathbb{R}, \tau \in \mathcal{T}_{t}$ and $\nu \in \mathcal{U}_{\text {unco }}^{t}$, there exists $\rho \in \mathcal{T}_{t}$ such that

$$
\mathbb{P}(Y(\rho)<g(X(\rho)) \mid B)>0
$$

for $B \subset A:=\{Y(\tau)<w(\tau, X(\tau))\}$ satisfying $B \in \mathcal{F}_{\tau}^{t}$ and $\mathbb{P}(B)>0$, where $X:=X_{t, x}^{\nu}$ and $Y:=Y_{t, x, y}^{\nu}$. Define

$$
\begin{aligned}
M(\cdot) & =Y(\cdot)-\int_{\tau}^{\cdot} K(s) d s, V(s)=w(s, X(s)), \Gamma(s)=(Y(s)-V(s)) \mathbb{1}_{A}, \text { where } \\
K(s) & :=\mu_{Y}(s, X(s), Y(s), \nu(s))+\int_{E} b^{\top}\left(s, X(s-), Y(s-), \nu_{1}(s), \nu_{2}(s, e), e\right) m(d e), \\
\widetilde{K}(s) & :=\mu_{Y}(s, X(s), V(s), \nu(s))+\int_{E} b^{\top}\left(s, X(s-), V(s-), \nu_{1}(s), \nu_{2}(s, e), e\right) m(d e) .
\end{aligned}
$$

It is easy to see that $M$ is a martingale after $\tau$. Due to the facts that $A \in \mathcal{F}_{\tau}^{t}$ and $d V(s)=k e^{k s} d s$, we further know

$$
\mathbb{1}_{A}\left(Y(\cdot)-V(\cdot)+\int_{\tau}^{\cdot} k e^{k s}-K(s) d s\right) \text { is a super-martingale after } \tau \text {. }
$$

Since Assumption 3.5.4 holds and $\mu_{Y}(t, x, y, u)+\int_{E} b^{\top}(t, x, y, u(e), e) m(d e)$ is nondecreasing in $y$,

$$
\widetilde{K}(s) \leq \mu_{Y}\left(s, X(s), e^{k s}, \nu(s)\right)+\int_{E} b^{\top}\left(s, X(s-), e^{k s}, \nu_{1}(s), \nu_{2}(s, e), e\right) m(d e) \leq 2 C e^{k s}
$$

Therefore, it follows from (3.5.5) and the inequality above that

$$
\left.\widetilde{M}(\cdot):=\mathbb{1}_{A}\left(Y(\cdot)-V(\cdot)-\int_{\tau}^{\cdot} \xi(s) d s\right)\right) \text { is a super-martingale after } \tau,
$$

where $\xi(s):=K(s)-\widetilde{K}(s)$. Since $\widetilde{M}(\tau)<0$ on $B$, there exists a non-null set $F \subset B$ such that $\widetilde{M}(\rho)<0$ on $F$ for any $\rho \in \mathcal{T}_{\tau}$. By the definition of $\widetilde{M}$ in (3.5.6), we get

$$
\Gamma(\rho)<\mathbb{1}_{A} \int_{\tau}^{\rho} \xi(s) d s \text { on } F .
$$

Therefore,

$$
\Gamma^{+}(\rho) \leq \mathbb{1}_{A} \int_{\tau}^{\rho} \xi^{+}(s) d s \leq \int_{\tau}^{\rho} L_{0} \Gamma^{+}(s) d s \text { on } F
$$


By Grönwall's Inequality, $\Gamma^{+}(\tau)=0$ implies that $\Gamma^{+}(\rho)=0$ on $F$. More precisely, for $\omega \in F(\mathbb{P}-$ a.s. $), \Gamma^{+}(s)(\omega)=0$ for $s \in[\tau(\omega), \rho(\omega)]$. This implies that we can replace the inequalities with equalities in (3.5.8). Therefore, by $(3.5 .7), \Gamma(\rho)<0$ on $F$, which yields $\mathbb{P}(Y(\rho)<g(X(\rho)) \mid B)>0$.

\subsubsection{The equivalence results}

Let $T$ be a finite time horizon, given a general probability space $(\Omega, \mathcal{F}, \mathbb{P})$ endowed with a filtration $\mathbb{F}=\left\{\mathcal{F}_{t}\right\}_{0 \leq t \leq T}$ satisfying the usual conditions. Let $\mathcal{T}_{t}$ be the set of $\mathbb{F}$-stopping times valued in $[t, T]$. In particular, let $\mathcal{T}:=\mathcal{T}_{0}$. We assume that $\mathcal{F}_{0}$ is trivial. Let $\mathcal{U}$ be the collection of all $\mathbb{F}$-predictable processes valued in $U \subset \mathbb{R}^{k}$ and $\left\{G^{\nu}, \nu \in \mathcal{U}\right\}$ be a collection of bounded, right-continuous processes valued in $\mathbb{R}$. Given $(t, \nu) \in[0, T] \times \mathcal{U}$, we consider the following two problems:

$$
V_{\text {unco }}^{\nu}(t)=\operatorname{ess} \inf _{\mu \in \mathcal{U}(t, \nu)} \operatorname{esssup} \underset{\tau \in \mathcal{T}_{t}}{ } \mathbb{E}\left[G^{\mu}(\tau) \mid \mathcal{F}_{t}\right]
$$

and

$$
V_{\text {co }}^{\nu}(t)=\operatorname{ess} \sup _{\mu \in \mathcal{U}(t, \nu)} \operatorname{esssup} \operatorname{su\mathcal {T}}_{\tau} \mathbb{E}\left[G^{\mu}(\tau) \mid \mathcal{F}_{t}\right]
$$

where $\mathcal{U}(t, \nu)=\{\mu \in \mathcal{U}, \mu=\nu$ on $[0, t] \mathbb{P}-$ a.s. $\}$.

Lemma 3.5.6. Given $t \in[0, T]$ and $\nu \in \mathcal{U}_{t}$, let $\mathcal{M}$ be any family of martingales which satisfies the following:

For any $\mu \in \mathcal{U}(t, \nu)$, there exists an $M \in \mathcal{M}$ such that

$$
\underset{\tau \in \mathcal{T}_{t}}{\operatorname{esssup}} \mathbb{E}\left[G^{\mu}(\tau) \mid \mathcal{F}_{t}\right]+M(\rho)-M(t) \geq G^{\mu}(\rho) \text { for all } \rho \in \mathcal{T}_{t}
$$

Then $V_{\text {unco }}^{\nu}(t)=Y_{\text {unco }}^{\nu}(t)$, where

$$
\begin{gathered}
Y_{\text {unco }}^{\nu}(t)=\operatorname{essinf}\left\{Y \in L^{1}\left(\Omega, \mathcal{F}_{t}, \mathbb{P}\right) \mid \exists(M, \mu) \in \mathcal{M} \times \mathcal{U}(t, \nu)\right. \text { such that } \\
\left.Y+M(\rho)-M(t) \geq G^{\mu}(\rho) \text { for all } \rho \in \mathcal{T}_{t}\right\} .
\end{gathered}
$$


Proof. (1) $Y_{\text {unco }}^{\nu}(t) \geq V_{\text {unco }}^{\nu}(t):$ Fix $Y \in L^{1}\left(\Omega, \mathcal{F}_{t}, \mathbb{P}\right)$ and $(M, \mu) \in \mathcal{M} \times \mathcal{U}(t, \nu)$ such that

$$
Y+M(\rho)-M(t) \geq G^{\mu}(\rho) \text { for all } \rho \in \mathcal{T}_{t}
$$

By taking the conditional expectation, we get that

$$
Y \geq \mathbb{E}\left[G^{\mu}(\rho) \mid \mathcal{F}_{t}\right] \text { for all } \rho \in \mathcal{T}_{t}
$$

which implies that $Y \geq V_{\text {unco }}^{\nu}(t)$. Therefore, $Y_{\text {unco }}^{\nu}(t) \geq V_{\text {unco }}^{\nu}(t)$.

(2) $V_{\text {unco }}^{\nu}(t) \geq Y_{\text {unco }}^{\nu}(t)$ : we get from (3.5.11) that for each $\mu \in \mathcal{U}(t, \nu)$, there exists an $M \in \mathcal{M}$ such that

$$
\operatorname{esssup}_{\tau \in \mathcal{T}_{t}} \mathbb{E}\left[G^{\mu}(\tau) \mid \mathcal{F}_{t}\right]+M(\rho)-M(t) \geq G^{\mu}(\rho) \text { for all } \rho \in \mathcal{T}
$$

This implies that

$$
\underset{\tau \in \mathcal{T}_{t}}{\operatorname{esssup}} \mathbb{E}\left[G^{\mu}(\tau) \mid \mathcal{F}_{t}\right] \geq Y_{\text {unco }}^{\nu}(t),
$$

which further implies $V_{\text {unco }}^{\nu}(t) \geq Y_{\text {unco }}^{\nu}(t)$.

Lemma 3.5.7. Let $\mathcal{M}$ be any family of martingales which satisfies the following:

For any $\nu \in \mathcal{U}$ and $\rho \in \mathcal{T}$, there exists $M \in \mathcal{M}$ such that $G^{\nu}(\rho)=M(\rho)$.

Then for each $(t, \nu) \in[0, T] \times \mathcal{U}, V_{c o}^{\nu}(t)=Y_{c o}^{\nu}(t)$, where

$$
\begin{gathered}
Y_{c o}^{\nu}(t)=\operatorname{esssup}\left\{Y \in L^{1}\left(\Omega, \mathcal{F}_{t}, \mathbb{P}\right) \mid \exists(M, \mu, \rho) \in \mathcal{M} \times \mathcal{U}(t, \nu) \times \mathcal{T}_{t},\right. \\
\text { such that } \left.Y+M(\rho)-M(t) \leq G^{\mu}(\rho)\right\} .
\end{gathered}
$$

Proof. (1) $Y_{\text {co }}^{\nu}(t) \leq V_{\text {co }}^{\nu}(t):$ Fix $Y \in L^{1}\left(\Omega, \mathcal{F}_{t}, \mathbb{P}\right)$ and $(M, \mu, \rho) \in \mathcal{M} \times \mathcal{U}(t, \nu) \times \mathcal{T}_{t}$ such that

$$
Y+M(\rho)-M(t) \leq G^{\mu}(\rho) .
$$


Then by taking the conditional expectation, we get that

$$
Y \leq \mathbb{E}\left[G^{\mu}(\rho) \mid \mathcal{F}_{t}\right] \leq V_{\mathrm{co}}^{\nu}(t)
$$

which implies that $Y_{\text {co }}^{\nu}(t) \leq V_{\text {co }}^{\nu}(t)$.

(2) $Y_{\mathrm{co}}^{\nu}(t) \geq V_{\text {co }}^{\nu}(t)$ : we get from (3.5.11) that for each $\mu \in \mathcal{U}(t, \nu)$ and $\rho \in \mathcal{T}_{t}$, there exists $M \in \mathcal{M}$ such that

$$
\mathbb{E}\left[G^{\mu}(\rho) \mid \mathcal{F}_{t}\right]+M(\rho)-M(t)=G^{\mu}(\rho)
$$

In particular,

$$
\mathbb{E}\left[G^{\mu}(\rho) \mid \mathcal{F}_{t}\right]+M(\rho)-M(t) \leq G^{\mu}(\rho)
$$

Therefore, $\mathbb{E}\left[G^{\mu}(\rho) \mid \mathcal{F}_{t}\right] \leq Y_{\text {co }}^{\nu}(t)$, which implies $V_{\text {co }}^{\nu}(t) \leq Y_{\text {co }}^{\nu}(t)$.

Remark 3.5.8. It is clear that a collection of martingales which satisfies (3.5.12) always exists. In particular, one can take

$$
\mathcal{M}_{\mathrm{co}}=\left\{\left\{\mathbb{E}\left[G^{\nu}(\rho) \mid \mathcal{F}_{t}\right]\right\}_{0 \leq t \leq T}, \nu \in \mathcal{U}, \rho \in \mathcal{T}\right\}
$$




\section{CHAPTER IV}

\section{Stochastic Perron for Stochastic Target Games}

\subsection{Outline of this chapter}

In this chapter, we will analyze a stochastic target game as described in Chapter I. This chapter is organized as follows. In Section 4.2, we introduce the setup of the stochastic target game as used in [16], the related HJB equation and the definitions of the stochastic semi-solutions (our conceptual contribution). The technical contribution of this chapter is given in Section 4.3, where we characterize the infimum (supremum) of the stochastic super-solutions (sub-solutions) as the viscosity sub-solution (super-solution) of the HJB equation. A viscosity comparison argument concludes that the value function is the unique bounded continuous viscosity solution of the HJB equation. Finally, we obtain the dynamic programming principle as a byproduct. Some technical results are deferred to the appendix (Section 4.4).

\subsection{The setup}

\subsubsection{The value function}

Let $\Omega$ be the space of continuous functions $\omega:[0, T] \rightarrow \mathbb{R}^{d}$ and let $\mathbb{P}$ be the Wiener measure on $\Omega$. We will denote by $W$ the canonical process on $\Omega$, i.e. $W_{t}(\omega)=\omega_{t}$, and by $\mathbb{F}=\left(\mathcal{F}_{s}\right)_{0 \leq s \leq T}$ the augmented filtration generated by $W$. For $0 \leq t \leq T$ let $\mathbb{F}^{t}=\left(\mathcal{F}_{s}^{t}\right)_{0 \leq s \leq T}$ be the augmented filtration generated by $\left(W_{s}-W_{t}\right)_{s \geq t}$. By 
convention, $\mathcal{F}_{s}^{t}$ is trivial for $s \leq t$.

We denote by $\mathcal{U}^{t}$ (resp. $\left.\mathcal{A}^{t}\right)$ the collection of all $\mathbb{F}^{t}$-predictable processes in $\mathbb{L}^{p}(\mathbb{P} \otimes$ $\left.\lambda_{L}\right)$ with values in a given Borel subset $U$ (resp. bounded set $A$ ) of $\mathbb{R}^{d}$, where $p \geq 2$ and $\lambda_{L}$ is the Lebesgue measure on $\mathbb{R}$. Define

$$
\mathbb{D}:=[0, T] \times \mathbb{R}^{d}, \quad \mathbb{D}_{i}:=[0, T) \times \mathbb{R}^{d}, \quad \mathbb{D}_{T}:=\{T\} \times \mathbb{R}^{d}
$$

Given $(t, x, y) \in \mathbb{D} \times \mathbb{R}$ and $(u, \alpha) \in \mathcal{U}^{t} \times \mathcal{A}^{t}$, consider the following SDEs.

$$
\begin{aligned}
& d X(s)=\mu_{X}\left(s, X(s), \alpha_{s}\right) d s+\sigma_{X}\left(s, X(s), \alpha_{s}\right) d W_{s} \\
& d Y(s)=\mu_{Y}\left(s, X(s), Y(s), u_{s}, \alpha_{s}\right) d s+\sigma_{Y}\left(s, X(s), Y(s), u_{s}, \alpha_{s}\right) d W_{s}
\end{aligned}
$$

with initial data $(X(t), Y(t))=(x, y)$.

Assumption 4.2.1. The coefficients $\mu_{X}, \mu_{Y}, \sigma_{X}$ and $\sigma_{Y}$ are continuous in all variables and take values in $\mathbb{R}^{d}, \mathbb{R}, \mathbb{R}^{d}$ and $\mathbb{M}^{d}:=\mathbb{R}^{d \times d}$, respectively. There exists $K>0$ such that for all $(t, x, y, u, a) \in \mathbb{D} \times \mathbb{R} \times U \times A$ and $\left(t^{\prime}, x^{\prime}, y^{\prime}\right) \in \mathbb{D} \times \mathbb{R}$,

$$
\begin{gathered}
\left|\mu_{X}(t, x, a)-\mu_{X}\left(t^{\prime}, x^{\prime}, a\right)\right|+\left|\sigma_{X}(t, x, a)-\sigma_{X}\left(t^{\prime}, x^{\prime}, a\right)\right| \leq K\left(\left|t-t^{\prime}\right|+\left|x-x^{\prime}\right|\right), \\
\left|\mu_{X}(t, x, a)\right|+\left|\sigma_{X}(t, x, a)\right| \leq K, \\
\left|\mu_{Y}(t, x, y, u, a)-\mu_{Y}\left(t, x, y^{\prime}, u, a\right)\right|+\left|\sigma_{Y}(t, x, y, u, a)-\sigma_{Y}\left(t, x, y^{\prime}, u, a\right)\right| \leq K\left|y-y^{\prime}\right|, \\
\left|\mu_{Y}(t, x, y, u, a)\right|+\left|\sigma_{Y}(t, x, y, u, a)\right| \leq K(1+|u|+|y|) .
\end{gathered}
$$

This assumption ensures that the stochastic differential equations given in (4.2.1) are well-posed. Denote the solutions to (4.2.1) by $\left(X_{t, x}^{\alpha}, Y_{t, x, y}^{u, \alpha}\right)$. Let $t \leq T$. We say that a map $\mathfrak{u}: \mathcal{A}^{t} \rightarrow \mathcal{U}^{t}, \alpha \mapsto \mathfrak{u}[\alpha]$ is a $t$-admissible strategy if it is non-anticipating in the sense that

$$
\left\{\omega \in \Omega:\left.\alpha(\omega)\right|_{[t, s]}=\left.\alpha^{\prime}(\omega)\right|_{[t, s]}\right\} \subset\left\{\omega \in \Omega:\left.\mathfrak{u}[\alpha](\omega)\right|_{[t, s]}=\left.\mathfrak{u}\left[\alpha^{\prime}\right](\omega)\right|_{[t, s]}\right\} \text {-a.s. }
$$

for all $s \in[t, T]$ and $\alpha, \alpha^{\prime} \in \mathcal{A}^{t}$, where $\left.\right|_{[t, s]}$ indicates the restriction to the interval $[t, s]$. We denote by $\mathfrak{U}(t)$ the collection of all $t$-admissible strategies; moreover, we 
write $Y_{t, x, y}^{\mathfrak{u}, \alpha}$ for $Y_{t, x, y}^{\mathfrak{u}[\alpha], \alpha}$. Then we can introduce the value function of the stochastic target game,

$$
v(t, x):=\inf \left\{y \in \mathbb{R}: \exists \mathfrak{u} \in \mathfrak{U}(t) \text { s.t. } Y_{t, x, y}^{\mathfrak{u}, \alpha}(T) \geq g\left(X_{t, x}^{\alpha}(T)\right) \text {-a.s. } \forall \alpha \in \mathcal{A}^{t}\right\}
$$

where $g: \mathbb{R}^{d} \rightarrow \mathbb{R}$ is a bounded continuous function. We also need to define strategies starting at a family of stopping times. Let $\mathcal{T}_{t}$ be the set of $\mathbb{F}^{t}$-stopping times valued in $[t, T]$.

Definition 4.2.2 (Non-anticipating family of stopping times). Let $\left\{\tau^{\alpha}\right\}_{\alpha \in \mathcal{A}^{t}} \subset \mathcal{T}_{t}$ be a family of stopping times. This family is $t$-non-anticipating if

$$
\begin{aligned}
& \left\{\omega \in \Omega:\left.\alpha(\omega)\right|_{[t, s]}=\left.\alpha^{\prime}(\omega)\right|_{[t, s]}\right\} \subset \\
& \left\{\omega \in \Omega: t \leq \tau^{\alpha}(\omega)=\tau^{\alpha^{\prime}}(\omega) \leq s\right\} \cup\left\{\omega \in \Omega: s<\tau^{\alpha}(\omega), s<\tau^{\alpha^{\prime}}(\omega)\right\} \text {-a.s. }
\end{aligned}
$$

Denote the set of $t$-non-anticipating families of stopping times by $\mathbb{T}_{t}$.

We will use $\left\{\tau^{\alpha}\right\}$ for short to represent $\left\{\tau^{\alpha}\right\}_{\alpha \in \mathcal{A}^{t}}$, which will always denote a t-nonanticipating family of stopping times.

Definition 4.2.3 (Strategies starting at a non-anticipating family of stopping times). Fix $t$ and let $\left\{\tau^{\alpha}\right\} \in \mathbb{T}_{t}$. We say that a map $\mathfrak{u}: \mathcal{A}^{t} \rightarrow \mathcal{U}^{t}, \alpha \mapsto \mathfrak{u}[\alpha]$ is a $\left(t,\left\{\tau^{\alpha}\right\}\right)$ admissible strategy if it is non-anticipating in the sense that

$$
\begin{gathered}
\left\{\omega \in \Omega:\left.\alpha(\omega)\right|_{[t, s]}=\left.\alpha^{\prime}(\omega)\right|_{[t, s]}\right\} \subset\left\{\omega \in \Omega: s<\tau^{\alpha}(\omega), s<\tau^{\alpha^{\prime}}(\omega)\right\} \cup \\
\left\{\omega \in \Omega: t \leq \tau^{\alpha}(\omega)=\tau^{\alpha^{\prime}}(\omega) \leq s,\left.\mathfrak{u}[\alpha](\omega)\right|_{\left[\tau^{\alpha}(\omega), s\right]}=\left.\mathfrak{u}\left[\alpha^{\prime}\right](\omega)\right|_{\left[\tau^{\alpha^{\prime}}(\omega), s\right]}\right\} \text {-a.s. }
\end{gathered}
$$

for all $s \in[t, T]$ and $\alpha, \alpha^{\prime} \in \mathcal{A}^{t}$, denoted by $\mathfrak{u} \in \mathfrak{U}\left(t,\left\{\tau^{\alpha}\right\}\right)$.

It is clear that, in the Definition 4.2 .3 if we set $\tau^{\alpha}=t$ for all $\alpha$, then $\mathfrak{U}\left(t,\left\{\tau^{\alpha}\right\}\right)$ is then same as $\mathfrak{U}(t)$. Hence, the above definitions are consistent. 
Definition 4.2.4 (Concatenation). Let $\alpha_{1}, \alpha_{2} \in \mathcal{A}^{t}, \tau \in \mathcal{T}_{t}$ is a stopping time. The concatenation of $\alpha_{1}, \alpha_{2}$ is defined as follows:

$$
\alpha_{1} \otimes_{\tau} \alpha_{2}:=\alpha_{1} \mathbb{1}_{[t, \tau)}+\alpha_{2} \mathbb{1}_{[\tau, T]}
$$

The concatenation of elements in $\mathcal{U}^{t}$ is defined in the similar fashion.

Lemma 4.2.5. Fix $t$ and let $\left\{\tau^{\alpha}\right\} \in \mathbb{T}_{t}$. For $\mathfrak{u} \in \mathfrak{U}(t)$ and $\tilde{\mathfrak{u}} \in \mathfrak{U}\left(t,\left\{\tau^{\alpha}\right\}\right)$, define $\mathfrak{u}_{*}[\alpha]:=\mathfrak{u}[\alpha] \otimes_{\tau^{\alpha}} \tilde{\mathfrak{u}}[\alpha]$. Then $\mathfrak{u}_{*} \in \mathfrak{U}(t)$. For the rest of the chapter, we will use $\mathfrak{u} \otimes_{\tau^{\alpha}} \tilde{\mathfrak{u}}[\alpha]$ to represent $\mathfrak{u}[\alpha] \otimes_{\tau^{\alpha}} \tilde{\mathfrak{u}}[\alpha]$.

Proof. It is obvious that $u_{*}$ maps $\mathcal{A}^{t}$ to $\mathcal{U}^{t}$. Let us check the non-anticipativity of the map. For any fixed $s \in[t, T]$ and $\alpha, \alpha^{\prime} \in \mathcal{A}^{t}, \omega^{\prime} \in\left\{\omega \in \Omega:\left.\alpha(\omega)\right|_{[t, s]}=\left.\alpha^{\prime}(\omega)\right|_{[t, s]}\right\}$, by Definition 4.2 .2 ,

$$
\omega^{\prime} \in\left\{t \leq \tau^{\alpha}=\tau^{\alpha^{\prime}} \leq s\right\} \cup\left\{s<\tau^{\alpha}, s<\tau^{\alpha^{\prime}}\right\} \text {-a.s. }
$$

(i) If $\omega^{\prime} \in\left\{t \leq \tau^{\alpha}=\tau^{\alpha^{\prime}} \leq s\right\}$, by definition of $\mathfrak{u}_{*}$,

$$
\begin{aligned}
\left.\mathfrak{u}_{*}[\alpha]\left(\omega^{\prime}\right)\right|_{[t, s]} & =\left.\mathfrak{u}[\alpha]\left(\omega^{\prime}\right) \mathbb{1}_{\left[t, \tau^{\alpha}\left(\omega^{\prime}\right)\right)}\right|_{[t, s]}+\left.\tilde{\mathfrak{u}}[\alpha]\left(\omega^{\prime}\right) \mathbb{1}_{\left[\tau^{\alpha}\left(\omega^{\prime}\right), T\right]}\right|_{[t, s]}, \\
\left.\mathfrak{u}_{*}\left[\alpha^{\prime}\right]\left(\omega^{\prime}\right)\right|_{[t, s]} & =\left.\mathfrak{u}\left[\alpha^{\prime}\right]\left(\omega^{\prime}\right) \mathbb{1}_{\left[t, \alpha^{\left.\alpha^{\prime}\left(\omega^{\prime}\right)\right)}\right.}\right|_{[t, s]}+\left.\tilde{\mathfrak{u}}\left[\alpha^{\prime}\right]\left(\omega^{\prime}\right) \mathbb{1}_{\left[\tau^{\alpha^{\prime}}\left(\omega^{\prime}\right), T\right]}\right|_{[t, s]}
\end{aligned}
$$

Since $\tau^{\alpha}\left(\omega^{\prime}\right)=\tau^{\alpha^{\prime}}\left(\omega^{\prime}\right), \mathfrak{u} \in \mathfrak{U}(t)$ and by Definition 4.2 .3 , we know

$$
\omega^{\prime} \in\left\{\omega \in \Omega:\left.\mathfrak{u}[\alpha](\omega)\right|_{[t, s]}=\left.\mathfrak{u}\left[\alpha^{\prime}\right](\omega)\right|_{[t, s]}\right\} \text {-a.s. }
$$

(ii) If $\omega^{\prime} \in\left\{s<\tau^{\alpha}, s<\tau^{\alpha^{\prime}}\right\}$, using definition of $\mathfrak{u}_{*}$,

$$
\left.\mathfrak{u}_{*}[\alpha]\left(\omega^{\prime}\right)\right|_{[t, s]}=\left.\mathfrak{u}[\alpha]\left(\omega^{\prime}\right)\right|_{[t, s]},\left.\mathfrak{u}_{*}\left[\alpha^{\prime}\right]\left(\omega^{\prime}\right)\right|_{[t, s]}=\left.\mathfrak{u}\left[\alpha^{\prime}\right]\left(\omega^{\prime}\right)\right|_{[t, s]}
$$

Since $\omega^{\prime} \in\left\{\omega \in \Omega:\left.\alpha(\omega)\right|_{[t, s]}=\left.\alpha^{\prime}(\omega)\right|_{[t, s]}\right\}$ and $\mathfrak{u} \in \mathfrak{U}(t)$, then

$$
\omega^{\prime} \in\left\{\omega \in \Omega:\left.\mathfrak{u}_{*}[\alpha](\omega)\right|_{[t, s]}=\left.\mathfrak{u}_{*}\left[\alpha^{\prime}\right](\omega)\right|_{[t, s]}\right\} \text {-a.s. }
$$




\subsubsection{The Hamilton-Jacobi-Bellman equation}

Before introducing the HJB equation, we make an assumption which was also assumed by [16] on the set-valued map

$$
N(t, x, y, z, a):=\left\{u \in U: \sigma_{Y}(t, x, y, u, a)=z\right\} \text { for }(t, x, y, z, a) \in \mathbb{D} \times \mathbb{R} \times \mathbb{R}^{d} \times A
$$

Assumption 4.2.6. $u \mapsto \sigma_{Y}(t, x, y, u, a)$ is invertible. More precisely, there exists a measurable map $\hat{u}: \mathbb{D} \times \mathbb{R} \times \mathbb{R}^{d} \times A \rightarrow U$ such that $N=\{\hat{u}\}$. Moreover, the map $\hat{u}(\cdot, a)$ is continuous for each $a \in A$.

Let us define for $(t, x, y, p, M) \in \mathbb{D} \times \mathbb{R} \times \mathbb{R}^{d} \times \mathbb{M}^{d}$,

$$
H(t, x, y, p, M):=\sup _{a \in A} H^{a}(t, x, y, p, M)
$$

where

$$
\begin{gathered}
\mu_{Y}^{\hat{u}}(t, x, y, z, a):=\mu_{Y}(t, x, y, \hat{u}(t, x, y, z, a), a) \text { and } \\
H^{a}(t, x, y, p, M):=-\mu_{Y}^{\hat{u}}\left(t, x, y, \sigma_{X}(t, x, a) p, a\right)+\mu_{X}^{\top}(t, x, a) p+\frac{1}{2} \operatorname{Tr}\left[\sigma_{X} \sigma_{X}^{\top}(t, x, a) M\right]
\end{gathered}
$$

The HJB equation is

$$
\begin{aligned}
\partial_{t} \varphi(t, x)+H\left(t, x, \varphi, D \varphi, D^{2} \varphi\right) & =0 \quad \text { on } \quad \mathbb{D}_{i}, \\
\varphi & =g \quad \text { on } \quad \mathbb{D}_{T} .
\end{aligned}
$$

\subsubsection{Stochastic solutions}

We will introduce weak solution concepts to the HJB equation that are stable under minimization and maximization respectively and envelope the value function $v$ of the stochastic target game.

Definition 4.2.7 (Stochastic super-solutions). A function $w:[0, T] \times \mathbb{R}^{d} \rightarrow \mathbb{R}$ is called a stochastic super-solution of (4.2.4) if 
1. It is bounded, continuous and $w(T, \cdot) \geq g(\cdot)$

2. For $(t, x, y) \in \mathbb{D} \times \mathbb{R},\left\{\tau^{\alpha}\right\} \in \mathbb{T}_{t}$ and $\mathfrak{u} \in \mathfrak{U}(t)$, there exists a strategy $\tilde{\mathfrak{u}} \in$ $\mathfrak{U}\left(t,\left\{\tau^{\alpha}\right\}\right)$ such that for any $\alpha \in \mathcal{A}^{t}$ and $\rho \in \mathcal{T}_{t}$ satisfying $\tau^{\alpha} \leq \rho \leq T$, we have

$$
Y(\rho) \geq w(\rho, X(\rho)) \mathbb{P}-\text { a.s. } \quad \text { on } \quad\left\{Y\left(\tau^{\alpha}\right)>w\left(\tau^{\alpha}, X\left(\tau^{\alpha}\right)\right)\right\}
$$

Here $X:=X_{t, x}^{\alpha}$ and $Y:=Y_{t, x, y}^{\mathfrak{u} \otimes_{\tau} \alpha \tilde{u}[\alpha], \alpha}$

The set of stochastic super-solutions is denoted by $\mathbb{U}^{+}$. Assume it is nonempty and $v^{+}:=\inf _{w \in \mathbb{U}^{+}} w$. For any stochastic super-solution $w$, choose $\tau^{\alpha}=t$ for all $\alpha$ and $\rho=T$, then there exists $\tilde{\mathfrak{u}} \in \mathfrak{U}(t)$ such that, for any $\alpha \in \mathcal{A}^{t}$,

$$
Y_{t, x, y}^{\tilde{\mathfrak{u}}, \alpha}(T) \geq w\left(T, X_{t, x}^{\alpha}(T)\right) \geq g\left(X_{t, x}^{\alpha}(T)\right) \quad \mathbb{P}-\text { a.s. } \quad \text { on } \quad\{y>w(t, x)\} .
$$

Hence, $y>w(t, x)$ implies $y \geq v(t, x)$ from (4.2.2). This gives $w \geq v$ and $v^{+} \geq v$. Similarly, we could define the stochastic sub-solutions.

Definition 4.2.8 (Stochastic sub-solutions). A function $w:[0, T] \times \mathbb{R}^{d} \rightarrow \mathbb{R}$ is called a stochastic sub-solution of (4.2.4) if

1. It is bounded, continuous and $w(T, \cdot) \leq g(\cdot)$,

2. For fixed $(t, x, y) \in \mathbb{D} \times \mathbb{R}$ and $\left\{\tau^{\alpha}\right\} \in \mathbb{T}_{t}$, for any $\mathfrak{u} \in \mathfrak{U}(t), \alpha \in \mathcal{A}^{t}$, there exists $\widetilde{\alpha} \in \mathcal{A}^{t}$ (may depend on $\mathfrak{u}, \alpha$ and $\tau^{\alpha}$ ) such that for each stopping time $\rho \in \mathcal{T}_{t}$, $\tau^{\alpha} \leq \rho \leq T$ with the simplifying notation $X:=X_{t, x}^{\alpha}, Y:=Y_{t, x, y}^{\mathfrak{u}, \alpha \otimes_{\tau}^{\alpha} \widetilde{\alpha}}$, we have

$$
\mathbb{P}(Y(\rho)<w(\rho, X(\rho)) \mid B)>0,
$$

for any $B \subset\left\{Y\left(\tau^{\alpha}\right)<w\left(\tau^{\alpha}, X\left(\tau^{\alpha}\right)\right\}, B \in \mathcal{F}_{\tau^{\alpha}}^{t}\right.$ and $\mathbb{P}(B)>0$.

The set of stochastic sub-solutions is denoted by $\mathbb{U}^{-}$. Assume it is nonempty and let $v^{-}:=\sup _{w \in \mathbb{U}^{-}} w$. For any stochastic sub-solution $w$, choose $\tau^{\alpha}=t$ for all $\alpha$ and 
$\rho=T$. Hence for any $\mathfrak{u} \in \mathfrak{U}(t)$, there exists $\widetilde{\alpha} \in \mathcal{A}^{t}$, such that

$$
\mathbb{P}\left(Y_{t, x, y}^{\mathfrak{u}, \widetilde{\alpha}}(T)<w\left(T, X_{t, x}^{\widetilde{\alpha}}(T)\right) \leq g\left(X_{t, x}^{\widetilde{\alpha}}(T)\right) \mid y<w(t, x)\right)>0
$$

Hence, $y<w(t, x)$ implies $y \leq v(t, x)$ from (4.2.2). This gives $w \leq v$ and $v^{-} \leq v$. As a result we have,

$$
v^{-} \triangleq \sup _{w \in \mathbb{U}^{-}} w \leq v \leq \inf _{w \in \mathbb{U}^{+}} w \triangleq v^{+}
$$

We will show in Section 4.3 that under some suitable assumptions, $v^{+}$and $v^{-}$are viscosity sub- and super-solutions of (4.2.4), respectively.

\subsubsection{Additional technical assumptions.}

We will need to make some more technical assumptions as in [16].

Assumption 4.2.9. The map $(t, x, y, z) \in \mathbb{D} \times \mathbb{R} \times \mathbb{R}^{d} \mapsto \mu_{Y}^{\hat{u}}(t, x, y, z, a)$ is Lipschitz continuous, uniformly in $a \in A$, and $(y, z) \in \mathbb{R} \times \mathbb{R}^{d} \mapsto \mu_{Y}^{\hat{u}}(t, x, y, z, a)$ has linear growth, uniformly in $(t, x, a) \in \mathbb{D} \times A$.

For the derivation of the super-solution property of $v^{-}$, we will impose a condition on the growth of $\mu_{Y}$ relative to $\sigma_{Y}$.

\section{Assumption 4.2.10.}

$$
\sup _{u \in U} \frac{\left|\mu_{Y}(\cdot, u, \cdot)\right|}{1+\left\|\sigma_{Y}(\cdot, u, \cdot)\right\|} \quad \text { is locally bounded, }
$$

where $\|\cdot\|$ is the Euclidean norm.

In (4.2.5) we implicitly assumed that the sets $\mathbb{U}^{+}$and $\mathbb{U}^{-}$are nonempty. The assumptions we made already imply that $\mathbb{U}^{+}$is not empty, but the same may not be true for $\mathbb{U}^{-}$is not empty.

Assumption 4.2.11. The collection $\mathbb{U}^{-}$is not empty. 


\subsubsection{The nonemptiness of $\mathbb{U}^{+}$and $\mathbb{U}^{-}$}

In this subsection, we discuss the nonemptiness of $\mathbb{U}^{+}$and $\mathbb{U}^{-}$. As the next result shows, the assumptions above already guarantee that $\mathbb{U}^{+}$is not empty.

Proposition 4.2.12. Under Assumptions 4.2.1, 4.2.6 and 4.2.9 the collection $\mathbb{U}^{+}$ is not empty.

Proof. See the Appendix.

In the above proposition the assumptions made can be replaced by the following natural assumption (although this is not the route we will take):

Assumption 4.2.13. There exists $\mathbf{u} \in U$ such that

$$
\mu_{Y}(t, x, y, \mathbf{u}, a)=0, \sigma_{Y}(t, x, y, \mathbf{u}, a)=0
$$

for all $(t, x, y, a) \in \mathbb{D}_{i} \times \mathbb{R} \times A$. (In these equations the right-hand-sides are denoted by just 0 for simplicity, but they in fact are collections of 0's matching the dimension on the left-hand-side.)

In the context of super-hedging in mathematical finance, in which $Y$ represents the wealth of an investor and $X$ the stock price, and $g\left(X_{T}\right)$ a financial contract, the last assumption is equivalent to allowing the investor not to trade in the risky assets.

Proposition 4.2.14. Under Assumptions 4.2.1 and 4.2.13 the collection $\mathbb{U}^{+}$is not empty.

Proof. Choose the strategy $\tilde{u}[\alpha]=\mathbf{u}$. For any given $\left\{\tau^{\alpha}\right\} \in \mathbb{T}_{t}$, we have $\tilde{\mathfrak{u}} \in$ $\mathcal{U}\left(t,\left\{\tau^{\alpha}\right\}\right)$ and from Assumption 4.2.13, it holds for any $\mathfrak{u} \in \mathfrak{U}(t)$ that

$$
Y_{t, x, y}^{\mathfrak{u} \otimes_{\tau} \alpha \tilde{\mathfrak{u}}[\alpha], \alpha}(\rho)=Y_{t, x, y}^{\mathfrak{u} \otimes_{\tau} \tilde{\mathfrak{u}}[\alpha], \alpha}\left(\tau^{\alpha}\right), \forall \alpha \in \mathcal{A}^{t} \text { and } \rho \in \mathcal{T}_{t} \text { such that } \tau^{\alpha} \leq \rho \leq T
$$


From the boundedness of $g$, there exists an $C$, such that $g(x)<C$. Now take $w(t, x) \equiv C$, which clearly satisfies the first condition in Definition 4.2.7. On the other hand, on $\left\{Y\left(\tau^{\alpha}\right)>w\left(\tau^{\alpha}, X\left(\tau^{\alpha}\right)\right)\right\}$, we clearly have that $\{Y(\rho)>w(\rho, X(\rho))\}$ for any $\rho$ such that $\tau^{\alpha} \leq \rho \leq T$, which gives the second condition in Definition 4.2.7.

Proposition 4.2.15. If in addition to Assumptions 4.2.1 there exists a $\in A$ such that $\mu_{Y}(t, x, y, u, a)=0, \sigma_{Y}(t, x, y, u, a)=0$ for all $(t, x, y, u) \in \mathbb{D}_{i} \times \mathbb{R} \times U$, then $\mathbb{U}^{-}$ is not empty.

Proof. The proof is similar to that of Proposition 4.2.14.

The additional assumption in the latter proposition is not very reasonable. Below we introduce an alternative assumption.

Assumption 4.2.16. $\frac{\left|\mu_{Y}\right|}{\left\|\sigma_{Y}\right\|}$ is bounded on $N=\left\{(t, x, y, u, a): \sigma_{Y}(t, x, y, u, a) \neq 0\right\}$.

Proposition 4.2.17. Under Assumptions 4.2.1, 4.2.6, 4.2.13, and 4.2.16, $\mathbb{U}^{-}$is not empty.

Proof. See the Appendix.

\subsection{The main results}

Before we state and prove the main theorem, we need some preparatory lemmas.

Lemma 4.3.1. The set of stochastic super-solutions (resp. stochastic sub-solutions) is upwards (resp. downwards) directed, i.e.,

1. If $w_{1}, w_{2} \in \mathbb{U}^{+}$, then $w_{1} \wedge w_{2} \in \mathbb{U}^{+}$;

2. If $w_{1}, w_{2} \in \mathbb{U}^{-}$, then $w_{1} \vee w_{2} \in \mathbb{U}^{-}$. 
Proof. This lemma is in the spirit of Lemma 3.7 in [30]. Here we only sketch the proof for (1). For $w_{1}, w_{2} \in \mathbb{U}^{+}$, let $w=w_{1} \wedge w_{2}$. Clearly $w$ is bounded, continuous and $w(T, x) \geq g(x)$. For fixed $(t, x, y) \in \mathbb{D}_{i} \times \mathbb{R}$ and $\left\{\tau^{\alpha}\right\} \in \mathbb{T}_{t}$, let $\mathfrak{u}_{1}$ and $\mathfrak{u}_{2}$ are the strategies starting at $\left\{\tau^{\alpha}\right\}$ for $w_{1}$ and $w_{2}$, respectively. Let

$$
\mathfrak{u}[\alpha]=\mathfrak{u}_{1}[\alpha] \mathbb{1}_{\left\{w_{1}\left(\tau^{\alpha}, X\left(\tau^{\alpha}\right)\right)<w_{2}\left(\tau^{\alpha}, X\left(\tau^{\alpha}\right)\right)\right\}}+\mathfrak{u}_{2}[\alpha] \mathbb{1}_{\left\{w_{1}\left(\tau^{\alpha}, X\left(\tau^{\alpha}\right)\right) \geq w_{2}\left(\tau^{\alpha}, X\left(\tau^{\alpha}\right)\right)\right\}}
$$

It is easy to show that $\mathfrak{u}$ works for $w$ in the definition of stochastic super-solutions.

Lemma 4.3.2. There exists a non-increasing sequence $\mathbb{U}^{+} \ni w_{n} \searrow v^{+}$and a nondecreasing sequence $\mathbb{U}^{-} \ni v_{n} \nearrow v^{-}$.

Proof. The proof of the lemma follows directly from Proposition 4.1 in [8].

Lemma 4.3.3. Given $f: X \times Y \subset \mathbb{R}^{p} \times \mathbb{R}^{q} \rightarrow \mathbb{R}$, define $F(x):=\sup _{y \in Y} f(x, y)$. If $x \rightarrow f(x, y)$ is continuous, uniformly in $y$ and $F(x)<\infty$ for all $x \in X$, then $x \rightarrow F(x)$ is continuous.

The lemma above is easy to check and we omit the proof.

Theorem 4.3.4. Let Assumptions 4.2.1 and 4.2.6 hold.

1. If in addition $g$ is USC and Assumption 4.2.9 holds, the function $v^{+}$is a bounded USC viscosity sub-solution of (4.2.4).

2. On the other hand if $g$ is LSC and Assumptions 4.2.10 and 4.2.11 hold, the function $v^{-}$is a bounded LSC viscosity super-solution of (4.2.4).

Proof. Step 1. ( $v^{+}$is the viscosity sub-solution). Due to Proposition 4.2.12, $v^{+}$is well-defined. We will first show the interior viscosity sub-solution property and then demonstrate the boundary condition.

1.1 The interior sub-solution property: Let $\left(t_{0}, x_{0}\right) \in \mathbb{D}_{i}$ and $\varphi \in C^{1,2}(\mathbb{D})$ 
be such that $\varphi$ strictly touches $v^{+}$from above at $\left(t_{0}, x_{0}\right)$. Assume, by contradiction, that

$$
\partial_{t} \varphi\left(t_{0}, x_{0}\right)+H\left(t_{0}, x_{0}, \varphi\left(t_{0}, x_{0}\right), D \varphi\left(t_{0}, x_{0}\right), D^{2} \varphi\left(t_{0}, x_{0}\right)\right)<0 \text {. }
$$

From the uniform continuity of $\mu_{X}$ and $\sigma_{X}$ in Assumption 4.2.1, the uniform continuity of $\mu_{Y}^{\hat{u}}$ in Assumption 4.2.9 and the smoothness of $\varphi$, the map $(t, x, y, a) \rightarrow$ $H^{a}\left(t, x, y, D \varphi(t, x), D^{2} \varphi(t, x)\right)$ is uniformly continuous in $(t, x, y)$. Hence the map

$$
(t, x, y) \rightarrow H\left(t, x, y, D \varphi(t, x), D^{2} \varphi(t, x)\right)
$$

is continuous due to Lemma 4.3.3. This implies that there exist $\varepsilon>0$ and $\delta>0$ such that for all $(t, x) \in \bar{B}_{\varepsilon}\left(t_{0}, x_{0}\right)$ and $|y-\varphi(t, x)| \leq \delta$,

$$
\partial_{t} \varphi(t, x)+H\left(t, x, y, D \varphi(t, x), D^{2} \varphi(t, x)\right)<0 .
$$

On the compact set $\mathbb{T}:=\bar{B}_{\varepsilon}\left(t_{0}, x_{0}\right)-B_{\varepsilon / 2}\left(t_{0}, x_{0}\right)$, we have that $\varphi>v^{+}$and the min of $\varphi-v^{+}$is attained since $v^{+}$is USC. Therefore, $\varphi>v^{+}+\eta$ on $\mathbb{T}$ for some $\eta>0$. Since $w_{n} \searrow v^{+}$, a Dini type argument shows that, for large enough $n$ we have $\varphi>w_{n}+\eta / 2$ on $\mathbb{T}$ and $\varphi>w_{n}-\delta$ on $\bar{B}_{\varepsilon / 2}\left(t_{0}, x_{0}\right)$. For simplicity, fix such an $n$ and denote $w=w_{n}$. For $\kappa \in\left(0, \frac{\eta}{2} \wedge \delta\right)$, define

$$
w^{\kappa}:=\left\{\begin{array}{l}
(\varphi-\kappa) \wedge w \text { on } \bar{B}_{\varepsilon}\left(t_{0}, x_{0}\right), \\
w \text { outside } \bar{B}_{\varepsilon}\left(t_{0}, x_{0}\right)
\end{array}\right.
$$

Obviously, $w^{\kappa}$ is continuous and bounded. Since $w^{\kappa}\left(t_{0}, x_{0}\right)<v^{+}\left(t_{0}, x_{0}\right)$, we would obtain a contradiction if we can show $w^{\kappa} \in \mathbb{U}^{+}$.

Fix $t,\left\{\tau^{\alpha}\right\} \in \mathbb{T}_{t}$ and $\mathfrak{u} \in \mathfrak{U}(t)$. We need to construct a strategy $\tilde{\mathfrak{u}} \in \mathfrak{U}\left(t,\left\{\tau^{\alpha}\right\}\right)$ in the definition of stochastic super-solutions for $w^{\kappa}$. This can be done as follows: since $w$ is a stochastic super-solution, there exists an "optimal" strategy $\tilde{\mathfrak{u}}_{1}$ in the Definition 4.2.7 for $w$ starting at $\left\{\tau^{\alpha}\right\}$. We will construct $\tilde{\mathfrak{u}}$ in two steps: 
(i) $w^{\kappa}\left(\tau^{\alpha}, X_{t, x}^{\alpha}\left(\tau^{\alpha}\right)\right)=w\left(\tau^{\alpha}, X_{t, x}^{\alpha}\left(\tau^{\alpha}\right)\right)$ : set $\tilde{\mathfrak{u}}=\tilde{\mathfrak{u}}_{1}$;

(ii) $w^{\kappa}\left(\tau^{\alpha}, X_{t, x}^{\alpha}\left(\tau^{\alpha}\right)\right)<w\left(\tau^{\alpha}, X_{t, x}^{\alpha}\left(\tau^{\alpha}\right)\right)$ : In this case we necessarily start inside the ball. Let $\bar{Y}$ be the unique strong solution (which is thanks in particular to Assumption 4.2.9) of the equation

$$
\begin{aligned}
\bar{Y}(l)= & Y_{t, x, y}^{\mathfrak{u}, \alpha}\left(\tau^{\alpha}\right)+\int_{\tau^{\alpha}}^{\tau^{\alpha} \vee l} \mu_{Y}^{\hat{u}}\left(s, X_{t, x}^{\alpha}(s), \bar{Y}(s), \sigma_{X}\left(s, X_{t, x}^{\alpha}(s), \alpha_{s}\right) D \varphi\left(s, X_{t, x}^{\alpha}(s)\right), \alpha_{s}\right) d s \\
& +\int_{\tau^{\alpha}}^{\tau^{\alpha} \vee l} \sigma_{X}\left(s, X_{t, x}^{\alpha}(s), \alpha_{s}\right) D \varphi\left(s, X_{t, x}^{\alpha}(s)\right) d W_{s}, \quad l \geq \tau^{\alpha}
\end{aligned}
$$

for any $\mathfrak{u} \in \mathfrak{U}(t)$ and $\alpha \in \mathcal{A}^{t}$ and set $\bar{Y}(s)=Y_{t, x, y}^{\mathfrak{u}, \alpha}(s)$ for $s<\tau^{\alpha}$. Define

$$
\tilde{\mathfrak{u}}_{0}:=\tilde{\mathfrak{u}}_{0}[\alpha](s)=\hat{u}\left(s, X_{t, x}^{\alpha}(s), \bar{Y}(s), \sigma_{X}\left(s, X_{t, x}^{\alpha}(s), \alpha_{s}\right) D \varphi\left(s, X_{t, x}^{\alpha}(s)\right), \alpha_{s}\right)
$$

Let $\theta_{1}^{\alpha}$ is the first exit time of $\left(s, X_{t, x}^{\alpha}(s)\right)$ after $\tau^{\alpha}$ from $B_{\varepsilon / 2}\left(t_{0}, x_{0}\right)$ and $\theta_{2}^{\alpha}$ be the first time after $\tau^{\alpha}$ when $\left|\bar{Y}(s)-\varphi\left(s, X_{t, x}^{\alpha}(s)\right)\right| \geq \delta$. More, precisely,

$$
\theta_{1}^{\alpha}:=\inf \left\{s \in\left[\tau^{\alpha}, T\right]:\left(s, X_{t, x}^{\alpha}(s)\right) \notin B_{\varepsilon / 2}\left(t_{0}, x_{0}\right)\right\}
$$

and

$$
\theta_{2}^{\alpha}:=\inf \left\{s \in\left[\tau^{\alpha}, T\right]:\left|\bar{Y}(s)-\varphi\left(s, X_{t, x}^{\alpha}(s)\right)\right| \geq \delta\right\}
$$

Let $\theta^{\alpha}=\theta_{1}^{\alpha} \wedge \theta_{2}^{\alpha}$. We know that $\left\{\theta^{\alpha}\right\} \in \mathbb{T}_{t}$ from Example 1 in [4]. We will set $\tilde{\mathfrak{u}}$ to be $\tilde{\mathfrak{u}}_{0}$ until $\theta^{\alpha}$. Starting at $\theta^{\alpha}$, we will then follow the strategy $\mathfrak{u}^{\theta} \in \mathfrak{U}\left(t,\left\{\theta^{\alpha}\right\}\right)$ which is "optimal" for $w$.

In summary, (i) and (ii) together gave us the following strategy:

$$
\tilde{\mathfrak{u}}[\alpha]=\left(\mathbb{1}_{A} \tilde{\mathfrak{u}}_{1}[\alpha]+\mathbb{1}_{A^{c}}\left(\tilde{\mathfrak{u}}_{0}[\alpha] \mathbb{1}_{\left[t, \theta^{\alpha}\right)}+\mathfrak{u}^{\theta}[\alpha] \mathbb{1}_{\left[\theta^{\alpha}, T\right]}\right)\right) \mathbb{1}_{\left[\tau^{\alpha}, T\right]},
$$

where

$$
A=\left\{w^{\kappa}\left(\tau^{\alpha}, X_{t, x}^{\alpha}\left(\tau^{\alpha}\right)\right)=w\left(\tau^{\alpha}, X_{t, x}^{\alpha}\left(\tau^{\alpha}\right)\right)\right\}
$$

We note that $\tilde{\mathfrak{u}}_{0} \in \mathfrak{U}(t)$ by the pathwise uniqueness of $X$ 's, $Y$ 's and $\bar{Y}$ 's equations. Then applying Lemma 4.2.5, $\tilde{\mathfrak{u}}_{0}[\alpha] \mathbb{1}_{\left[t, \theta^{\alpha}\right)}+\mathfrak{u}^{\theta}[\alpha] \mathbb{1}_{\left[\theta^{\alpha}, T\right]} \in \mathfrak{U}(t)$. Since $\tilde{\mathfrak{u}}_{1} \in \mathfrak{U}\left(t,\left\{\tau^{\alpha}\right\}\right)$, 
by Definition 4.2 .3 , it follows that $\tilde{\mathfrak{u}} \in \mathfrak{U}\left(t,\left\{\tau^{\alpha}\right\}\right)$ by the pathwise uniqueness of $X$ 's equation. Now, let us show the above construction actually works. We need to show that for any $\rho \in \mathcal{T}_{t}$ such that $\tau^{\alpha} \leq \rho \leq T$,

$$
Y(\rho) \geq w^{\kappa}(\rho, X(\rho)) \mathbb{P}-\text { a.s. } \quad \text { on } \quad\left\{Y\left(\tau^{\alpha}\right)>w^{\kappa}\left(\tau^{\alpha}, X\left(\tau^{\alpha}\right)\right)\right\}
$$

where

$$
X:=X_{t, x}^{\alpha} \quad \text { and } \quad Y:=Y_{t, x, y}^{\mathfrak{u} \otimes_{\tau} \alpha \tilde{\mathfrak{u}}[\alpha], \alpha}
$$

Note that $\bar{Y}(s)=Y_{t, x, y}^{\mathfrak{u} \otimes_{\tau} \alpha \tilde{\mathfrak{u}}_{0}[\alpha], \alpha}(s)$ for $s \geq \tau^{\alpha}$ and

$$
Y=\mathbb{1}_{A} Y_{t, x, y}^{\mathfrak{u} \otimes_{\tau}^{\alpha} \tilde{\mathfrak{u}}_{1}[\alpha], \alpha}+\mathbb{1}_{A^{c}} Y_{t, x, y}^{\mathfrak{u} \otimes_{\tau^{\alpha}} \tilde{\mathfrak{u}}_{0}[\alpha], \alpha} \quad \text { for } \quad \tau^{\alpha} \leq s \leq \theta^{\alpha}
$$

We will carry out the proof in two steps:

(i) On the set $A \cap\left\{Y\left(\tau^{\alpha}\right)>w^{\kappa}\left(\tau^{\alpha}, X\left(\tau^{\alpha}\right)\right)\right\}$, we have $Y\left(\tau^{\alpha}\right)>w\left(\tau^{\alpha}, X\left(\tau^{\alpha}\right)\right)$. From (4.3.2) and the "optimality" of $\tilde{\mathfrak{u}}_{1}$ (for $w$ ), we know

$$
Y(\rho)=Y_{t, x, y}^{\mathfrak{u} \otimes_{\tau} \alpha \tilde{\mathfrak{u}}_{1}[\alpha], \alpha}(\rho) \geq w(\rho, X(\rho)) \geq w^{\kappa}(\rho, X(\rho)) \mathbb{P}-\text { a.s on the above set. }
$$

(ii) On the set $A^{c} \cap\left\{Y\left(\tau^{\alpha}\right)>w^{\kappa}\left(\tau^{\alpha}, X\left(\tau^{\alpha}\right)\right\}\right.$, by the definition of $\tilde{\mathfrak{u}}_{0}$ and (4.3.2), using Itô's Formula,

$$
Y\left(\cdot \wedge \theta^{\alpha}\right)-\varphi\left(\cdot \wedge \theta^{\alpha}, X\left(\cdot \wedge \theta^{\alpha}\right)\right)=Y\left(\tau^{\alpha}\right)-\varphi\left(\tau^{\alpha}, X\left(\tau^{\alpha}\right)\right)+\int_{\tau^{\alpha}}^{\cdot \wedge \theta^{\alpha}} \gamma(s) d s
$$

where

$$
\gamma(s):=-H^{\alpha_{s}}\left(s, X(s), Y(s), D \varphi(s, X(s)), D^{2} \varphi(s, X(s))\right)-\partial_{t} \varphi(s, X(s)),
$$

since the definition of $\hat{u}$ allows us to cancel the Brownian motion terms on the righthand-side. Obviously, on $\left[\tau^{\alpha}, \theta^{\alpha}\right], \gamma>0$. This implies that $Y\left(\cdot \wedge \theta^{\alpha}\right)-\varphi\left(\cdot \wedge \theta^{\alpha}, X(\cdot \wedge\right.$ $\left.\left.\theta^{\alpha}\right)\right)$ is non-decreasing on $\left[\tau^{\alpha}, T\right]$. Therefore,

$$
Y\left(\theta^{\alpha}\right)-\varphi\left(\theta^{\alpha}, X\left(\theta^{\alpha}\right)\right)+\kappa>Y\left(\tau^{\alpha}\right)-\varphi\left(\tau^{\alpha}, X\left(\tau^{\alpha}\right)\right)+\kappa>0
$$


As a result, on the one hand, we have

$$
0<\left(Y\left(\theta_{1}^{\alpha}\right)-\varphi\left(\theta_{1}^{\alpha}, X\left(\theta_{1}^{\alpha}\right)\right)+\kappa\right) \leq\left(Y\left(\theta_{1}^{\alpha}\right)-w\left(\theta_{1}^{\alpha}, X\left(\theta_{1}^{\alpha}\right)\right)\right) \quad \text { on } \quad\left\{\theta_{1}^{\alpha}<\theta_{2}^{\alpha}\right\}
$$

On the other hand,

$$
Y\left(\theta_{2}^{\alpha}\right)-\varphi\left(\theta_{2}^{\alpha}, X\left(\theta_{2}^{\alpha}\right)\right)=\delta \quad \text { on } \quad\left\{\theta_{1}^{\alpha} \geq \theta_{2}^{\alpha}\right\}
$$

Observe that the right-hand-side of the above expression cannot be $-\delta$ due to (4.3.3). Therefore,

$$
Y\left(\theta_{2}^{\alpha}\right)-w\left(\theta_{2}^{\alpha}, X\left(\theta_{2}^{\alpha}\right)\right)=\delta+\varphi\left(\theta_{2}^{\alpha}, X\left(\theta_{2}^{\alpha}\right)\right)-w\left(\theta_{2}^{\alpha}, X\left(\theta_{2}^{\alpha}\right)\right)>0 \text { on }\left\{\theta_{1}^{\alpha} \geq \theta_{2}^{\alpha}\right\}
$$

since $\varphi>w-\delta$ on $\bar{B}_{\varepsilon / 2}\left(t_{0}, x_{0}\right)$. Combining (4.3.4) and (4.3.5) we obtain

$$
Y\left(\theta^{\alpha}\right)-w\left(\theta^{\alpha}, X\left(\theta^{\alpha}\right)\right)>0 \quad \text { on } \quad A^{c} \cap\left\{Y\left(\tau^{\alpha}\right)>w^{\kappa}\left(\tau^{\alpha}, X^{\alpha}\right)\right\}
$$

It follows from this conclusion and the "optimality" of $\mathfrak{u}^{\theta}$ starting at $\left\{\theta^{\alpha}\right\}$ that

$$
Y\left(\rho \vee \theta^{\alpha}\right) \geq w\left(\rho \vee \theta^{\alpha}, X\left(\rho \vee \theta^{\alpha}\right)\right) \geq w^{\kappa}\left(\rho \vee \theta^{\alpha}, X\left(\rho \vee \theta^{\alpha}\right)\right)
$$

on $A^{c} \cap\left\{Y\left(\tau^{\alpha}\right)>w^{\kappa}\left(\tau^{\alpha}, X^{\alpha}\right)\right\}$. Also, since $Y\left(\cdot \wedge \theta^{\alpha}\right)-\varphi\left(\cdot \wedge \theta^{\alpha}, X\left(\cdot \wedge \theta^{\alpha}\right)\right)$ is nondecreasing on $\left[\tau^{\alpha}, T\right]$,

$$
Y\left(\rho \wedge \theta^{\alpha}\right)-\varphi\left(\rho \wedge \theta^{\alpha}, X\left(\rho \wedge \theta^{\alpha}\right)\right)+\kappa>0 \text { on } A^{c} \cap\left\{Y\left(\tau^{\alpha}\right)>w^{\kappa}\left(\tau^{\alpha}, X^{\alpha}\right)\right\}
$$

Therefore,

$$
Y\left(\rho \wedge \theta^{\alpha}\right)-w^{\kappa}\left(\rho \wedge \theta^{\alpha}, X\left(\rho \wedge \theta^{\alpha}\right)\right)>0 \text { on } A^{c} \cap\left\{Y\left(\tau^{\alpha}\right)>w^{\kappa}\left(\tau^{\alpha}, X^{\alpha}\right)\right\}
$$

From (4.3.6) and (4.3.7) we have

$$
Y(\rho)-w^{\kappa}(\rho, X(\rho)) \geq 0 \text { on } A^{c} \cap\left\{Y\left(\tau^{\alpha}\right)>w^{\kappa}\left(\tau^{\alpha}, X^{\alpha}\right)\right\}
$$




\subsection{The boundary condition:}

Step A: In this step we will assume that $\mu_{Y}^{\hat{u}}$ is non-decreasing in its y-variable.

Assume to the contrary that for some $x_{0} \in \mathbb{R}^{d}$, we have

$$
v^{+}\left(T, x_{0}\right)>g\left(x_{0}\right)
$$

Since $g$ is USC, then from (4.3.8) there exists $\varepsilon>0$ such that

$$
v^{+}\left(T, x_{0}\right)>g(x)+\varepsilon \text { for }\left|x-x_{0}\right| \leq \varepsilon \text {. }
$$

Choose $\varepsilon$ such that $\varepsilon<1$. Since $v^{+}$is USC, then $v^{+}$is bounded above on the compact set $\mathbb{T}=\overline{\mathcal{B}}_{\varepsilon}\left(T, x_{0}\right)-\mathcal{B}_{\varepsilon / 2}\left(T, x_{0}\right)$, where

$$
\mathcal{B}_{\varepsilon}\left(T, x_{0}\right)=\left\{(t, x) \in \mathbb{D}: \max \left\{|T-t|,\left|x-x_{0}\right|\right\}<\varepsilon\right\} .^{1}
$$

Choose $\beta>0$ small enough, such that

$$
v^{+}\left(T, x_{0}\right)+\frac{\varepsilon^{2}}{4 \beta}>\varepsilon+\sup _{\mathbb{T}} v^{+}(t, x) .
$$

By a Dini type argument there exists a $w \in \mathbb{U}^{+}$such that

$$
v^{+}\left(T, x_{0}\right)+\frac{\varepsilon^{2}}{4 \beta}>\varepsilon+\sup _{\mathbb{T}} w(t, x) .
$$

For $C>0$ let us denote

$$
\varphi^{\beta, C}(t, x)=v^{+}\left(T, x_{0}\right)+\frac{\left|x-x_{0}\right|^{2}}{\beta}+C(T-t)
$$

Hence, $D \varphi^{\beta, C}(t, x)=\frac{2\left(x-x_{0}\right)}{\beta}$ and $D^{2} \varphi^{\beta, C}(t, x)=\frac{2}{\beta} I_{d \times d}$. From Assumption 4.2.1,

$$
\left|\mu_{X}^{\top}(t, x, a) D \varphi^{\beta, C}(t, x)\right| \leq 2 K \frac{\left|x-x_{0}\right|}{\beta} \leq \frac{2 K}{\beta} \text { on } \overline{\mathcal{B}}_{\varepsilon}\left(T, x_{0}\right) \text { for } a \in A
$$

where we use $\varepsilon<1$. Similarly,

$$
\begin{gathered}
\left|\frac{1}{2} \operatorname{Tr}\left[\sigma_{X} \sigma_{X}^{\top}(t, x, a) D^{2} \varphi^{\beta, C}(t, x)\right]\right| \leq \frac{1}{2} K^{2} \frac{2 d}{\beta}=\frac{K^{2} d}{\beta} \\
\text { for }(t, x) \in \overline{\mathcal{B}}_{\varepsilon}\left(T, x_{0}\right) \text { and } a \in A,
\end{gathered}
$$

\footnotetext{
${ }^{1}$ Note that such a neighborhood is not the regular Euclidean ball. The definition of such a neighborhood is crucial for (4.3.15)
} 
where $d$ is the dimension of the space where the variable $x$ lives. From the linear growth condition of $\mu_{Y}^{\hat{u}}$ in Assumption 4.2.9, there exists $L>0$ such that for all $(t, x) \in \overline{\mathcal{B}}_{\varepsilon}\left(T, x_{0}\right)$ and $a \in A$,

$$
\begin{aligned}
& -\mu_{Y}^{\hat{u}}\left(t, x, \varphi^{\beta, 0}-\varepsilon, \sigma_{X}(t, x, a) D \varphi^{\beta, 0}, a\right) \\
\leq & L\left(1+\left|\varphi^{\beta, 0}(t, x)-\varepsilon\right|+\left|\sigma_{X}(t, x, a) D \varphi^{\beta, 0}(t, x)\right|\right) \\
\leq & L\left(1+v^{+}\left(T, x_{0}\right)+1 / \beta+1+2 K / \beta\right)
\end{aligned}
$$

Noting that $D \varphi^{\beta, C}(t, x)=D \varphi^{\beta, 0}(t, x)$, from the monotonicity assumption of $\mu_{Y}^{\hat{u}}$, we have

$$
-\mu_{Y}^{\hat{u}}\left(t, x, \varphi^{\beta, C}-\varepsilon, \sigma_{X}(t, x, a) D \varphi^{\beta, C}, a\right) \leq-\mu_{Y}^{\hat{u}}\left(t, x, \varphi^{\beta, 0}-\varepsilon, \sigma_{X}(t, x, a) D \varphi^{\beta, 0}, a\right) .
$$

The above equation with $(4.3 .11),(4.3 .12)$ and $(4.3 .13)$ implies that $H\left(\cdot, \varphi^{\beta, C}-\right.$ $\left.\varepsilon, D \varphi^{\beta, C}, D^{2} \varphi^{\beta, C}\right)(t, x)$ is bounded from above on $\overline{\mathcal{B}}_{\varepsilon}\left(T, x_{0}\right)$ and the bound is independent of $C$. Therefore, we can find $C>0$ large enough such that for $(t, x) \in \mathcal{B}_{\varepsilon}\left(T, x_{0}\right)$ and $y \geq \varphi^{\beta, C}(t, x)-\varepsilon$,

$$
\partial_{t} \varphi^{\beta, C}+H\left(\cdot, y, D \varphi^{\beta, C}, D^{2} \varphi^{\beta, C}\right)(t, x)<0
$$

where we used the monotonicity assumption of $\mu_{Y}^{\hat{u}}$. Making sure that $C \geq \varepsilon / 2 \beta$, we obtain from (4.3.10) that

$$
\varphi^{\beta, C} \geq \varepsilon+w \quad \text { on } \quad \mathbb{T} \text {. }
$$

Also,

$$
\varphi^{\beta, C}(T, x) \geq v^{+}\left(T, x_{0}\right)>g(x)+\varepsilon \text { for }\left|x-x_{0}\right| \leq \varepsilon .
$$

Now we can choose $\kappa<\varepsilon$ and define

$$
w^{\beta, C, \kappa}:=\left\{\begin{array}{l}
\left(\varphi^{\beta, C}-\kappa\right) \wedge w \text { on } \overline{\mathcal{B}}_{\varepsilon}\left(T, x_{0}\right), \\
w \text { outside } \overline{\mathcal{B}}_{\varepsilon}\left(T, x_{0}\right) .
\end{array}\right.
$$


From (4.3.16) and (4.3.17) it is easy to see that $w^{\beta, C, \kappa}(T, x) \geq g(x)$. By applying similar arguments as in Step 1.1, we can show that $w^{\beta, C, \kappa}$ is a stochastic supersolution with $w^{\beta, C, \kappa}\left(T, x_{0}\right)<v^{+}\left(T, x_{0}\right)$. This contradicts the definition of $v^{+}$.

Step B: We now turn to showing the same result for more general $\mu_{Y}^{\hat{u}}$ and follow a proof similar to that in [16]. Fix $c>0$ and define $\widetilde{Y}_{t, x, y}^{\mathfrak{u}, \alpha}$ as the strong solution of

$$
d \widetilde{Y}(s)=\tilde{\mu}_{Y}\left(s, X_{t, x}^{\alpha}(s), \widetilde{Y}(s), \mathfrak{u}[\alpha]_{s}, \alpha_{s}\right) d s+\tilde{\sigma}_{Y}\left(s, X_{t, x}^{\alpha}(s), \widetilde{Y}(s), \mathfrak{u}[\alpha]_{s}, \alpha_{s}\right) d W_{s}
$$

with initial data $\widetilde{Y}(t)=y$, where

$$
\begin{aligned}
& \tilde{\mu}_{Y}(t, x, y, u, a):=c y+e^{c t} \mu_{Y}\left(t, x, e^{-c t} y, u, a\right), \\
& \tilde{\sigma}_{Y}(t, x, y, u, a):=e^{c t} \sigma_{Y}\left(t, x, e^{-c t} y, u, a\right) .
\end{aligned}
$$

Hence, $\tilde{Y}_{t, x, y}^{\mathfrak{u}, \alpha}(s) e^{-c s}=Y_{t, x, y e^{-c t}}^{\mathfrak{u}, \alpha}(s)$ for any $s \in[t, T]$ by the strong uniqueness. Set $\tilde{g}(x):=e^{c T} g(x)$ and define

$$
\tilde{v}(t, x):=\inf \left\{y \in \mathbb{R}: \exists \mathfrak{u} \in \mathfrak{U}^{t} \text { s.t. } \widetilde{Y}_{t, x, y}^{\mathfrak{u}, \alpha}(T) \geq \tilde{g}\left(X_{t, x}^{\alpha}(T)\right) \text {-a.s. } \forall \alpha \in \mathcal{A}^{t}\right\}
$$

Therefore, $\tilde{v}(t, x)=e^{c t} v(t, x)$. Since $\mu_{Y}^{\hat{u}}$ has linear growth in its second argument $y$, one can choose large enough $c>0$ so that

$$
\tilde{\mu}_{Y}^{\hat{u}}:(t, x, y, z, a) \mapsto c y+e^{c t} \mu_{Y}^{\hat{u}}\left(t, x, e^{-c t} y, e^{-c t} z, a\right)
$$

is non-decreasing in its $y$-variable. This means that these dynamics satisfy the monotonicity assumption used in Step A above. Moreover, all the assumptions needed to apply Step A to this new problem are also satisfied. Let

$$
\begin{array}{r}
\widetilde{H}(t, x, y, p, M):=\sup _{a \in A}\left\{-c y-e^{c t} \mu_{Y}^{\tilde{u}}\left(t, x, e^{-c t} y, e^{-c t} \sigma_{X}(t, x, a) p, a\right)\right. \\
\left.+\mu_{X}^{\top}(t, x, a) p+\frac{1}{2} \operatorname{Tr}\left[\sigma_{X} \sigma_{X}^{\top}(t, x, a) M\right]\right\},
\end{array}
$$


where $\tilde{u}$ is defined like $\hat{u}$ but now in terms of $\tilde{\sigma}_{Y}$. We will denote by $\widetilde{U}^{+}$be the set of stochastic super-solutions of

$$
\begin{aligned}
\partial_{t} \varphi+\widetilde{H}\left(\cdot, \varphi, D \varphi, D^{2} \varphi\right) & =0 \text { on } \mathbb{D}_{i}, \\
\varphi & =\tilde{g} \text { on } \mathbb{D}_{T},
\end{aligned}
$$

and $\tilde{v}^{+}(t, x):=\inf _{w \in \widetilde{U}^{+}} w(t, x)$.

From step A, we know that $\tilde{v}^{+}$is a viscosity sub-solution of the above PDE. Since any function $w(t, x)$ is a stochastic super-solution of (4.2.4) if and only if $\tilde{w}(t, x)=e^{c t} w(t, x)$ is a stochastic super-solution of (4.3.20), it follows that $\tilde{v}^{+}(t, x)=$ $e^{c t} v^{+}(t, x)$. Now it is easy to conclude that $v^{+}$is a viscosity sub-solution of (4.2.4).

Step 2. ( $v^{-}$is the viscosity super-solution) Due to Assumption 4.2.11, $v^{-}$is well-defined. Next we will show that it satisfies the interior viscosity super-solution property followed by the boundary condition.

2.1 The interior super-solution property: Let $\left(t_{0}, x_{0}\right) \in \mathbb{D}_{i}$ and $\varphi \in C^{1,2}(\mathbb{D})$ be such that $\varphi$ strictly touches $v^{-}$from below at $\left(t_{0}, x_{0}\right)$. Assume by contradiction that

$$
\partial_{t} \varphi\left(t_{0}, x_{0}\right)+H\left(t_{0}, x_{0}, \varphi\left(t_{0}, x_{0}\right), D \varphi\left(t_{0}, x_{0}\right), D^{2} \varphi\left(t_{0}, x_{0}\right)\right)>0 \text {. }
$$

Hence there exists $a_{0} \in A$ such that

$$
\partial_{t} \varphi\left(t_{0}, x_{0}\right)+H^{u_{0}, a_{0}}\left(t_{0}, x_{0}, \varphi\left(t_{0}, x_{0}\right), D \varphi\left(t_{0}, x_{0}\right), D^{2} \varphi\left(t_{0}, x_{0}\right)\right)>0
$$

where $u_{0}=\hat{u}\left(t_{0}, x_{0}, \varphi\left(t_{0}, x_{0}\right), \sigma_{X}\left(t_{0}, x_{0}, a_{0}\right) D \varphi\left(t_{0}, x_{0}\right), D^{2} \varphi\left(t_{0}, x_{0}\right)\right)$ and

$$
H^{u, a}(t, x, y, p, M):=-\mu_{Y}(t, x, y, u, a)+\mu_{X}^{\top}(t, x, a) p+\frac{1}{2} \operatorname{Tr}\left[\sigma_{X} \sigma_{X}^{\top}(t, x, a) M\right] .
$$

From the continuity assumption on the coefficients in Assumption 4.2.1 and the continuity of $\hat{u}$ in Assumption 4.2.6, there exists $\varepsilon, \delta>0$ such that

$$
\begin{gathered}
\varphi_{t}+H^{u, a_{0}}\left(\cdot, y, D \varphi, D^{2} \varphi\right)>0 \forall(t, x) \in \bar{B}_{\varepsilon}\left(t_{0}, x_{0}\right) \text { and }(y, u) \in R \times U \text { s.t. } \\
|y-\varphi(t, x)| \leq \delta \quad \text { and } \quad\left|\sigma_{Y}\left(t, x, y, u, a_{0}\right)-\sigma_{X}\left(t, x, a_{0}\right) D \varphi(t, x)\right| \leq \delta .
\end{gathered}
$$


Now, on the compact set $\mathbb{T}=\bar{B}_{\varepsilon}\left(t_{0}, x_{0}\right)-B_{\varepsilon / 2}\left(t_{0}, x_{0}\right)$, we have that $\varphi<v^{-}$and the $\max$ of $\varphi-v^{-}$is attained since $v^{-}$is LSC. Therefore, $\varphi+\eta<v^{-}$on $\mathbb{T}$ for some $\eta>0$. Since $w_{n} \nearrow v^{-}$, a Dini type argument shows that, for large enough $n$ we have $\varphi+\eta / 2<w_{n}$ on $\mathbb{T}$ and $\varphi<w_{n}+\delta$ on $\bar{B}_{\varepsilon / 2}\left(t_{0}, x_{0}\right)$. For simplicity, fix such an $n$ and denote $w=w_{n}$. For $\kappa \in\left(0, \frac{\eta}{2} \wedge \delta\right)$,

$$
w^{\kappa}:=\left\{\begin{array}{l}
(\varphi+\kappa) \vee w \text { on } \bar{B}_{\varepsilon}\left(t_{0}, x_{0}\right) \\
w \text { outside } \bar{B}_{\varepsilon}\left(t_{0}, x_{0}\right)
\end{array}\right.
$$

Since $w^{\kappa}\left(t_{0}, x_{0}\right)>v^{-}\left(t_{0}, x_{0}\right)$, we obtain a contradiction if we can show that $w^{\kappa} \in \mathbb{U}^{-}$.

In order to do so, fix $t$ and $\left\{\tau^{\alpha}\right\} \in \mathbb{T}_{t}$. For a given $\mathfrak{u} \in \mathfrak{U}(t)$ and $\alpha \in \mathcal{A}^{t}$, we will construct an "optimal" $\widetilde{\alpha} \in \mathcal{A}^{t}$ in the definition of stochastic sub-solutions for $w^{\kappa}$. We will divide the construction into two cases:

(i) $w\left(\tau^{\alpha}, X\left(\tau^{\alpha}\right)\right)=w^{\kappa}\left(\tau^{\alpha}, X\left(\tau^{\alpha}\right)\right)$ : Since $w$ is a stochastic sub-solution, there exists an $\widetilde{\alpha}_{1}$ for $w$ in the definition which is "optimal" for the nature given $\mathfrak{u}, \alpha$ and $\tau^{\alpha}$. Let $\widetilde{\alpha}=\widetilde{\alpha}_{1}$.

(ii) $w\left(\tau^{\alpha}, X\left(\tau^{\alpha}\right)\right)<w^{\kappa}\left(\tau^{\alpha}, X\left(\tau^{\alpha}\right)\right)$ : Let

$$
\theta_{1}^{\alpha}:=\inf \left\{s \in\left[\tau^{\alpha}, T\right]:\left(s, X_{t, x}^{\alpha \otimes_{\tau^{\alpha} a_{0}}}(s)\right) \notin B_{\varepsilon / 2}\left(t_{0}, x_{0}\right)\right\} \wedge T
$$

and

$$
\theta_{2}^{\alpha}:=\inf \left\{s \in\left[\tau^{\alpha}, T\right]:\left|Y_{t, x, y}^{\mathfrak{u}, \alpha \otimes_{\tau^{\alpha} a_{0}}}(s)-\varphi\left(s, X_{t, x}^{\alpha \otimes_{\tau^{\alpha} a_{0}}}(s)\right)\right| \geq \delta\right\} \wedge T
$$

Denote $\theta^{\alpha}=\theta_{1}^{\alpha} \wedge \theta_{2}^{\alpha}$. Then let $\widetilde{\alpha}=a_{0}$ until $\theta^{\alpha}$. Starting from $\theta^{\alpha}$, choose $\widetilde{\alpha}=\alpha^{*}$, where the latter is "optimal" for nature given $\alpha$ and $\mathfrak{u}$ this time onward. In short, the above construction yields a candidate "optimal" control for $w^{\kappa}$ given by

$$
\widetilde{\alpha}=\left(\mathbb{1}_{A} \widetilde{\alpha}_{1}+\mathbb{1}_{A^{c}}\left(a_{0} \mathbb{1}_{\left[t, \theta^{\alpha}\right)}+\alpha^{*} \mathbb{1}_{\left[\theta^{\alpha}, T\right]}\right)\right) \mathbb{1}_{\left[\tau^{\alpha}, T\right]}
$$


where $A=\left\{w\left(\tau^{\alpha}, X_{t, x}^{\alpha}\left(\tau^{\alpha}\right)\right)=w^{\kappa}\left(\tau^{\alpha}, X_{t, x}^{\alpha}\left(\tau^{\alpha}\right)\right)\right\}$. We now check that what we constructed actually works. Let

$$
(X, Y)=\left(X_{t, x}^{\alpha \otimes_{\tau^{\alpha}} \widetilde{\alpha}}, Y_{t, x, y}^{\mathfrak{u}, \alpha \otimes_{\tau^{\alpha}} \widetilde{\alpha}}\right)
$$

Note that

$$
\begin{aligned}
& X(s)=\mathbb{1}_{A} X_{t, x}^{\alpha \otimes_{\tau^{\alpha}} \widetilde{\alpha}_{1}}(s)+\mathbb{1}_{A^{c}} X_{t, x}^{\alpha \otimes_{\tau^{\alpha} a_{0}}}(s) \quad \text { for } \tau^{\alpha} \leq s \leq \theta^{\alpha}, \\
& Y(s)=\mathbb{1}_{A} Y_{t, x, y}^{\mathfrak{u}, \alpha \otimes_{\tau} \alpha \widetilde{\alpha}_{1}}(s)+\mathbb{1}_{A^{c}} Y_{t, x, y}^{\mathfrak{u}, \alpha \otimes_{\tau^{\alpha} a_{0}}}(s) \quad \text { for } \tau^{\alpha} \leq s \leq \theta^{\alpha} .
\end{aligned}
$$

Define

$$
\begin{gathered}
E=\left\{Y\left(\tau^{\alpha}\right)<w^{\kappa}\left(\tau^{\alpha}, X\left(\tau^{\alpha}\right)\right)\right\}, \quad E_{0}=E \cap A, \quad E_{1}=E \cap A^{c}, \\
G=\left\{Y(\rho)<w^{\kappa}(\rho, X(\rho)\}, \quad G_{0}=\{Y(\rho)<w(\rho, X(\rho)\} .\right.
\end{gathered}
$$

Observe that

$$
E=E_{0} \cup E_{1}, E_{0} \cap E_{1}=\emptyset \text { and } G_{0} \subset G \text {. }
$$

The proof will be complete if we can show that $P(G \mid B)>0$ for any non-null set $B \subset E$. In fact, it suffices to show that $\mathbb{P}(G \cap B)>0$. Relying on the decomposition $\mathbb{P}(G \cap B)=\mathbb{P}\left(G \cap B \cap E_{0}\right)+\mathbb{P}\left(G \cap B \cap E_{1}\right)$ (recall that $\left.B \subset E\right)$, we will divide the proof into two steps:

(i) $\mathbb{P}\left(B \cap E_{0}\right)>0$ : Directly from the way $\widetilde{\alpha}_{1}$ is defined, the definition of the stochastic sub-solutions and $B \cap E_{0} \subset A$, we get

$$
\mathbb{P}\left(G_{0} \mid B \cap E_{0}\right)=\mathbb{P}\left(Y_{t, x, y}^{\mathfrak{u}, \alpha \otimes_{\tau^{\alpha}} \widetilde{\alpha}_{1}}(\rho)<w\left(\rho, X_{t, x}^{\alpha \otimes_{\tau^{\alpha}} \widetilde{\alpha}_{1}}(\rho)\right) \mid B \cap E_{0}\right)>0 .
$$

This further implies that $\mathbb{P}\left(G \cap B \cap E_{0}\right) \geq \mathbb{P}\left(G_{0} \cap B \cap E_{0}\right)>0$.

(ii) $\mathbb{P}\left(B \cap E_{1}\right)>0$ : From (4.3.22) and $B \cap E_{1} \subset A^{c}$,

$$
\mathbb{P}\left(Y\left(\theta^{\alpha}\right)<w^{\kappa}\left(\theta^{\alpha}, X\left(\theta^{\alpha}\right)\right) \mid B \cap E_{1}\right)=\mathbb{P}\left(Y_{t, x, y}^{\mathfrak{u}, \alpha \otimes_{\tau^{\alpha}} a_{0}}\left(\theta^{\alpha}\right)<w^{\kappa}\left(\theta^{\alpha}, X_{t, x}^{\alpha \otimes_{\tau^{\alpha} a_{0}}}\left(\theta^{\alpha}\right)\right) \mid B \cap E_{1}\right)
$$


The analysis in [16] shows that

$$
\Delta(s)=Y\left(s \wedge \theta^{\alpha}\right)-\left(\varphi\left(s \wedge \theta^{\alpha}, X\left(s \wedge \theta^{\alpha}\right)\right)+\kappa\right)
$$

is a super-martingale up to a change of measure. We summarize these arguments here. Let

$$
\begin{aligned}
\lambda(s):= & \sigma_{Y}\left(s, X(s), Y(s), \mathfrak{u}\left[a_{0}\right]_{s}, a_{0}\right)-\sigma_{X}\left(s, X(s), a_{0}\right) D \varphi(s, X(s)), \\
& \beta(s):=|\lambda(s)|^{-2} \lambda(s) \mathbb{1}_{\{|\lambda(s)|>\delta\}}\left(\partial_{t} \varphi(s, X(s))+\right. \\
& \left.H^{\mathfrak{u}\left[a_{0}\right]_{s}, a_{0}}\left(s, X(s), Y(s), D \varphi(s, X(s)), D^{2} \varphi(s, X(s))\right)\right) .
\end{aligned}
$$

From the definition of $\theta^{\alpha}$ and the regularity and growth conditions in Assumptions 4.2.1 and 4.2.10, $\beta$ is uniformly bounded on $\left[\tau^{\alpha}, \theta^{\alpha}\right]$. This ensures that the positive exponential local martingale $M$ defined by the SDE

$$
M(\cdot)=1+\int_{\tau^{\alpha}}^{\cdot \wedge \theta^{\alpha}} M(s) \beta_{s}^{\top} d W_{s}
$$

is a true martingale. An application of Itô's formula immediately implies that $M \Delta$ is a local super-martingale. By the definition of $\theta^{\alpha}, \Delta$ is bounded by $-\delta-\kappa$ from below and by $\delta-\kappa$ from above on $\left[\tau^{\alpha}, \theta^{\alpha}\right]$. Therefore, $M \Delta$ is bounded above by a martingale $2 M \delta$ and below by another martingale $-2 M \delta$. An application of Fatou's Lemma implies that $M \Delta$ is a super-martingale.

From the definition of $E_{1}$ and $w^{\kappa}, \Delta\left(\tau^{\alpha}\right)<0$ on $B \cap E_{1}$. The super-martingale property of $M \Delta$ implies that there exists a non-null set $H \subset B \cap E_{1}$ satisfying $H \in \mathcal{F}_{\tau^{\alpha}}^{t}$ such that $\Delta\left(\theta^{\alpha} \wedge \rho\right)<0$ on $H$. Therefore, from the decomposition

$$
\begin{gathered}
\Delta\left(\theta^{\alpha} \wedge \rho\right) \mathbb{1}_{H}=\left(Y\left(\theta_{1}^{\alpha}\right)-\varphi\left(\theta_{1}^{\alpha}, X\left(\theta_{1}^{\alpha}\right)\right)-\kappa\right) \mathbb{1}_{H \cap\left\{\theta_{1}^{\alpha}<\theta_{2}^{\alpha} \wedge \rho\right\}}+ \\
\left(Y\left(\theta_{2}^{\alpha}\right)-\varphi\left(\theta_{2}^{\alpha}, X\left(\theta_{2}^{\alpha}\right)\right)-\kappa\right) \mathbb{1}_{H \cap\left\{\theta_{2}^{\alpha} \leq \theta_{1}^{\alpha} \wedge \rho\right\}}+(Y(\rho)-\varphi(\rho, X(\rho))-\kappa) \mathbb{1}_{H \cap\left\{\rho<\theta^{\alpha}\right\}} .
\end{gathered}
$$

we see that

$$
Y\left(\theta_{1}^{\alpha}\right)-\varphi\left(\theta_{1}^{\alpha}, X\left(\theta_{1}^{\alpha}\right)\right)-\kappa<0 \quad \text { on } \quad H \cap\left\{\theta_{1}^{\alpha}<\theta_{2}^{\alpha} \wedge \rho\right\}
$$




$$
\begin{gathered}
Y\left(\theta_{2}^{\alpha}\right)-\varphi\left(\theta_{2}^{\alpha}, X\left(\theta_{2}^{\alpha}\right)\right)-\kappa<0 \quad \text { on } \quad H \cap\left\{\theta_{2}^{\alpha} \leq \theta_{1}^{\alpha} \wedge \rho\right\} \text { and } \\
Y(\rho)<\varphi(\rho, X(\rho))+\kappa \quad \text { on } \quad H \cap\left\{\rho<\theta^{\alpha}\right\} .
\end{gathered}
$$

On the one hand, on $H \cap\left\{\theta_{1}^{\alpha}<\theta_{2}^{\alpha} \wedge \rho\right\}, \varphi\left(\theta_{1}^{\alpha}, X\left(\theta_{1}^{\alpha}\right)\right)+\kappa<w\left(\theta_{1}^{\alpha}, X\left(\theta_{1}^{\alpha}\right)\right)$. Then from (4.3.23), we will have

$$
Y\left(\theta_{1}^{\alpha}\right)<w\left(\theta_{1}^{\alpha}, X\left(\theta_{1}^{\alpha}\right)\right) \quad \text { on } \quad H \cap\left\{\theta_{1}^{\alpha}<\theta_{2}^{\alpha} \wedge \rho\right\} .
$$

On the other hand, on $H \cap\left\{\theta_{2}^{\alpha} \leq \theta_{1}^{\alpha} \wedge \rho\right\}$, we get $Y\left(\theta_{2}^{\alpha}\right)-\varphi\left(\theta_{2}^{\alpha}, X\left(\theta_{2}^{\alpha}\right)\right)=-\delta$. (The right-hand-side can not be equal to $\delta$, otherwise (4.3.24) would be contradicted.) Recalling the fact that $\varphi<w+\delta$ on $\bar{B}_{\varepsilon / 2}\left(t_{0}, x_{0}\right)$, this observation gives that

$$
Y\left(\theta_{2}^{\alpha}\right)-w\left(\theta_{2}^{\alpha}, X\left(\theta_{2}^{\alpha}\right)\right)=(\varphi-w)\left(\theta_{2}^{\alpha}, X\left(\theta_{2}^{\alpha}\right)\right)-\delta<0 \text { on } H \cap\left\{\theta_{2}^{\alpha} \leq \theta_{1}^{\alpha} \wedge \rho\right\}
$$

We have obtained in (4.3.26) and (4.3.27) that

$$
Y\left(\theta^{\alpha}\right)<w\left(\theta^{\alpha}, X\left(\theta^{\alpha}\right)\right) \quad \text { on } \quad H \cap\left\{\theta^{\alpha} \leq \rho\right\} .
$$

Now from the definition of stochastic sub-solutions and of $\alpha^{*}$, we have that

$$
\mathbb{P}\left(G_{0} \mid H \cap\left\{\theta^{\alpha} \leq \rho\right\}\right)>0 \quad \text { if } \quad \mathbb{P}\left(H \cap\left\{\theta^{\alpha} \leq \rho\right\}\right)>0 .
$$

On the other hand, (4.3.25) implies that

$$
\mathbb{P}\left(G \mid H \cap\left\{\theta^{\alpha}>\rho\right\}\right)>0 \quad \text { if } \quad \mathbb{P}\left(H \cap\left\{\theta^{\alpha}>\rho\right\}\right)>0 .
$$

Since $\mathbb{P}(H)>0, G_{0} \subset G$, and $H \subset E_{1} \cap B$, (4.3.28) and (4.3.29) imply

$$
\mathbb{P}\left(G \cap E_{1} \cap B\right)>0 .
$$

\subsection{The boundary condition:}

Assume, on the contrary, that

$$
v^{-}\left(T, x_{0}\right)<g\left(x_{0}\right) \text { for some } x_{0} \in \mathbb{R}^{d} .
$$


From (4.3.30) and the lower semi-continuity of $g$, there exists $\varepsilon>0$ such that

$$
v^{-}\left(T, x_{0}\right)<g(x)-\varepsilon \text { for }\left|x-x_{0}\right| \leq \varepsilon
$$

Since $v^{-}$is LSC, $v^{-}$is bounded below on the compact set $\mathbb{T}=\overline{\mathcal{B}}_{\varepsilon}\left(T, x_{0}\right)-\mathcal{B}_{\varepsilon / 2}\left(T, x_{0}\right)$.

Choose $\beta>0$ small enough such that

$$
v^{-}\left(T, x_{0}\right)-\frac{\varepsilon^{2}}{4 \beta}<\inf _{\mathbb{T}} v^{-}(t, x)-\varepsilon
$$

By a Dini type argument, there exists $w \in \mathbb{U}^{-}$such that

$$
v^{-}\left(T, x_{0}\right)-\frac{\varepsilon^{2}}{4 \beta}<\inf _{\mathbb{T}} w(t, x)-\varepsilon
$$

For $C>0$, define

$$
\varphi^{\beta, C}(t, x)=v^{-}\left(T, x_{0}\right)-\frac{\left|x-x_{0}\right|^{2}}{\beta}-C(T-t) \text { for }(t, x) \in \mathbb{D} \text {. }
$$

For any $a_{0}$, we can choose large enough $C$ such that ${ }^{2}$

$$
\partial_{t} \varphi^{\beta, C}+H^{u_{0}, a_{0}}\left(\cdot, \varphi^{\beta, C}, D \varphi^{\beta, C}, D^{2} \varphi^{\beta, C}\right)>0 \text { on } \overline{\mathcal{B}}_{\varepsilon}\left(T, x_{0}\right)
$$

where $u_{0}=\hat{u}\left(T, x_{0}, \varphi\left(T, x_{0}\right), \sigma_{X}\left(T, x_{0}, a_{0}\right) D \varphi\left(T, x_{0}\right), a_{0}\right)$. Then from the continuity of the coefficients in Assumption 4.2.1 and the continuity of $\hat{u}$ in Assumption 4.2.6, for any $a_{0}$, and there exists a small enough $\delta>0$ such that

$$
\begin{aligned}
& \varphi_{t}^{\beta, C}+H^{u, a_{0}}\left(\cdot, y, D \varphi^{\beta, C}, D^{2} \varphi^{\beta, C}\right)>0 \forall(t, x) \in \overline{\mathcal{B}}_{\varepsilon}\left(T, x_{0}\right) \text { and }(y, u) \in R \times U \\
& \text { s.t. }\left|y-\varphi^{\beta, C}(t, x)\right| \leq \delta \quad \text { and } \quad\left|\sigma_{Y}\left(t, x, y, u, a_{0}\right)-\sigma_{X}\left(t, x, a_{0}\right) D \varphi^{\beta, C}(t, x)\right| \leq \delta
\end{aligned}
$$

Choosing $C>\varepsilon / 2 \beta$, we obtain from (4.3.32) that

$$
\varphi^{\beta, C} \leq w-\varepsilon \text { on } \mathbb{T}
$$

It also holds that

$$
\varphi^{\beta, C}(T, x) \leq v^{-}\left(T, x_{0}\right)<g(x)-\varepsilon \text { for }\left|x-x_{0}\right| \leq \varepsilon .
$$

\footnotetext{
${ }^{2}$ Similar analysis for (4.3.14) will guarantee that choosing $C$ is possible.
} 
For $\kappa \in(0, \varepsilon \wedge \delta)$, define

$$
w^{\beta, C, \kappa}:=\left\{\begin{array}{l}
\left(\varphi^{\beta, C}+\kappa\right) \vee w \text { on } \overline{\mathcal{B}}_{\varepsilon}\left(T, x_{0}\right) \\
w \text { outside } \overline{\mathcal{B}}_{\varepsilon}\left(T, x_{0}\right)
\end{array}\right.
$$

From (4.3.33) and (4.3.34), it is easy to see that $w^{\beta, C, \kappa}(T, x) \leq g(x)$. By applying arguments similar to those in Step 2.1, we can show that $w^{\beta, C, \kappa} \in \mathbb{U}^{-}$with $w^{\beta, C, \kappa}\left(T, x_{0}\right)>v^{-}\left(T, x_{0}\right)$. This contradicts the definition of $v^{-}$.

To characterize $v$ as the unique viscosity solution of (4.2.4), we need a comparison principle.

Proposition 4.3.5 (Comparison Principle). Under Assumptions 4.2.1, 4.2.6 and 4.2.9, the comparison principle for (4.2.4) holds. More precisely, suppose that $U$ (resp. $V$ ) be a bounded USC viscosity sub-solution (resp. LSC viscosity supersolution) to (4.2.4). If $U \leq V$ on $\mathbb{D}_{T}$, then $U \leq V$ on $\mathbb{D}$.

Proof. Step 1: Without loss of generality, assume that

$$
\exists \gamma>0 \text { such that } H(t, x, y, p, M)-H\left(t, x, y^{\prime}, p, M\right)<-\gamma\left(y-y^{\prime}\right)
$$

for all $y>y^{\prime}$. Otherwise, let $\widetilde{U}(t, x)=e^{c t} U(t, x)$ and $\widetilde{V}(t, x)=e^{c t} V(t, x)$. Then a straightforward calculation shows that $\widetilde{U}$ (resp. $\widetilde{V}$ ) is a sub-solution (resp. supersolution) to

$$
\begin{aligned}
\partial_{t} \varphi+\widetilde{H}\left(\cdot, \varphi, D \varphi, D^{2} \varphi\right) & =0 \quad \text { on } \quad \mathbb{D}_{i} \\
\varphi & =\tilde{g} \quad \text { on } \quad \mathbb{D}_{T}
\end{aligned}
$$

where $\widetilde{g}(x)=e^{c T} g(x)$ and $\widetilde{H}$ is the same as that in (4.3.19). We can choose $c$ large enough such that (4.3.35) holds for $\widetilde{H}$. In fact, from the Lipschitz continuity of $\mu_{Y}^{\hat{u}}$ 
in Assumption 4.2.9, for $y>y^{\prime}$,

$$
\begin{aligned}
& \widetilde{H}^{a}(t, x, y, p, M)-\widetilde{H}^{a}\left(t, x, y^{\prime}, p, M\right)=-c\left(y-y^{\prime}\right) \\
+ & e^{c t}\left(\mu_{Y}^{\tilde{u}}\left(t, x, e^{-c t} y^{\prime}, e^{-c t} \sigma_{X}(t, x, a) p, a\right)-\mu_{Y}^{\tilde{u}}\left(t, x, e^{-c t} y, e^{-c t} \sigma_{X}(t, x, a) p, a\right)\right) \\
\leq & -c\left(y-y^{\prime}\right)+e^{c t} L \cdot e^{-c t}\left(y-y^{\prime}\right) \\
= & -(c-L)\left(y-y^{\prime}\right),
\end{aligned}
$$

where $L$ is the Lipschitz constant and

$$
\begin{aligned}
\widetilde{H}^{a}(t, x, y, p, M): & =-c y-e^{c t} \mu_{Y}^{\tilde{u}}\left(t, x, e^{-c t} y, e^{-c t} \sigma_{X}(t, x, a) p, a\right) \\
& +\mu_{X}^{\top}(t, x, a) p+\frac{1}{2} \operatorname{Tr}\left[\sigma_{X} \sigma_{X}^{\top}(t, x, a) M\right] .
\end{aligned}
$$

Then $\gamma:=c-L>0$ for large enough c. Since $\widetilde{H}(\cdot)=\sup _{a \in A} \widetilde{H}^{a}(\cdot)$, equation (4.3.35) holds for $\widetilde{H}$.

Step 2: In this step, we claim that for large enough $\lambda, V_{\delta}:=V+\delta e^{-\lambda t}\left(1+|x|^{2}\right)$ is a LSC viscosity super-solution to (4.2.4) for $\delta>0$. Then, if we can show that $U-V_{\delta} \leq 0$ on $\mathbb{D}$ for all $\delta>0$, we will get the required result by sending $\delta$ to zero. Now we prove the above claim.

Obviously, the boundary condition is satisfied. Let $\varphi$ be a smooth function which strictly touches $V_{\delta}$ from below at $\left(t_{0}, x_{0}\right) \in \mathbb{D}_{i}$. Let $\varphi^{\delta}(t, x)=\varphi(t, x)-\delta e^{-\lambda t}\left(1+|x|^{2}\right)$ for all $(t, x) \in \mathbb{D}$. Then $V-\varphi^{\delta}$ has a strict minimum at $\left(t_{0}, x_{0}\right)$. Since $V$ is a viscosity super-solution, it holds that

$$
\partial_{t} \varphi^{\delta}(t, x)+H\left(t, x, \varphi^{\delta}(t, x), D \varphi^{\delta}(t, x), D^{2} \varphi^{\delta}(t, x)\right) \leq 0 \text { on } \mathbb{D}_{i} .
$$

Note that

$$
\begin{gathered}
\partial_{t} \varphi^{\delta}(t, x)=\partial_{t} \varphi(t, x)+\lambda \delta e^{-\lambda t}\left(1+|x|^{2}\right) \\
D \varphi^{\delta}(t, x)=D \varphi(t, x)-2 \delta e^{-\lambda t} x, D^{2} \varphi^{\delta}(t, x)=D^{2} \varphi(t, x)-2 \delta e^{-\lambda t} I_{d \times d} .
\end{gathered}
$$

Consider the difference of $H\left(t, x, \varphi^{\delta}, D \varphi^{\delta}, D^{2} \varphi^{\delta}\right)$ and $H\left(t, x, \varphi, D \varphi, D^{2} \varphi\right)$. From 
(4.3.38) and Assumption 4.2.1, we get that

$$
\begin{aligned}
& \left|\mu_{X}^{\top}(t, x, a) D \varphi(t, x)-\mu_{X}^{\top}(t, x, a) D \varphi^{\delta}(t, x)\right| \\
\leq & K\left|D \varphi(t, x)-D \varphi^{\delta}(t, x)\right|=2 K \delta e^{-\lambda t}|x| .
\end{aligned}
$$

Similarly,

$$
\left|\frac{1}{2} \operatorname{Tr}\left(\sigma_{X} \sigma_{X}^{\top}(t, x, a)\right) D^{2} \varphi(t, x)-\frac{1}{2} \operatorname{Tr}\left(\sigma_{X} \sigma_{X}^{\top}(t, x, a)\right) D^{2} \varphi^{\delta}(t, x)\right| \leq K^{2} d \delta e^{-\lambda t} .
$$

From the Lipschitz continuity of $\mu_{Y}^{\hat{u}}$ in Assumption 4.2.9,

$$
\begin{aligned}
& \left|\mu_{Y}^{\hat{u}}\left(t, x, \varphi, \sigma_{X}(t, x, a) D \varphi, a\right)-\mu_{Y}^{\hat{u}}\left(t, x, \varphi^{\delta}, \sigma_{X}(t, x, a) D \varphi^{\delta}, a\right)\right| \\
\leq & L\left(\delta e^{-\lambda t}\left(1+|x|^{2}\right)+2 K \delta e^{-\lambda t}|x|\right) .
\end{aligned}
$$

From (4.3.39), (4.3.40) and (4.3.41),

$$
\left|H\left(t, x, \varphi^{\delta}, D \varphi^{\delta}, D^{2} \varphi^{\delta}\right)-H\left(t, x, \varphi, D \varphi, D^{2} \varphi\right)\right| \leq \delta e^{-\lambda t}\left(1+|x|^{2}\right) \lambda^{*}
$$

where $\lambda^{*}:=L+L K+K^{2} d+K$. Taking $\lambda>\lambda^{*}$, we get that

$$
\begin{aligned}
& \partial_{t} \varphi(t, x)+H\left(t, x, \varphi(t, x), D \varphi(t, x), D^{2} \varphi(t, x)\right) \\
\leq & \partial_{t} \varphi^{\delta}(t, x)+H\left(t, x, \varphi^{\delta}, D \varphi^{\delta}, D^{2} \varphi^{\delta}\right)-\lambda \delta e^{-\lambda t}\left(1+|x|^{2}\right) \\
& +\left|H\left(t, x, \varphi^{\delta}, D \varphi^{\delta}, D^{2} \varphi^{\delta}\right)-H\left(t, x, \varphi, D \varphi, D^{2} \varphi\right)\right| \\
& \leq \partial_{t} \varphi^{\delta}+H\left(t, x, \varphi^{\delta}, D \varphi^{\delta}, D^{2} \varphi^{\delta}\right) \\
\leq & 0 .
\end{aligned}
$$

Step 3: In this step, we show that $U-V_{\delta} \leq 0$ on $\mathbb{D}$ for all $\delta>0$. From boundedness of $U$ and $V$,

$$
\lim _{|x| \rightarrow \infty} \sup _{[0, T]}\left(U-V_{\delta}\right)(t, x)=-\infty \text { for all } \delta>0 .
$$

This implies the supremum of $U-V_{\delta}$ on $\mathbb{D}$ is attained on $[0, T] \times \mathcal{O}$ for some open bounded set $\mathcal{O}$ of $\mathbb{R}^{d}$. Assume, for the sake of contradiction, that

$$
M^{*}:=\sup _{\mathbb{D}}\left(U-V_{\delta}\right)=\max _{[0, T) \times \mathcal{O}}\left(U-V_{\delta}\right)>0 .
$$


We will obtain a contradiction to the above equation. Take a bounded sequence $\left(t_{\varepsilon}, s_{\varepsilon}, x_{\varepsilon}, y_{\varepsilon}\right)_{\varepsilon}$ that maximizes $\Phi_{\varepsilon}$ on $[0, T]^{2} \times \mathbb{R}^{d} \times \mathbb{R}^{d}$ with $\Phi_{\varepsilon}=U(t, x)-V_{\delta}(s, y)-$ $\phi_{\varepsilon}(t, s, x, y)$ and $\phi_{\varepsilon}(t, s, x, y):=\frac{1}{2 \varepsilon}\left(|t-s|^{2}+|x-y|^{2}\right)$. By similar arguments in Theorem 4.4.4 in [26], we know that $\left(t_{\varepsilon}, s_{\varepsilon}, x_{\varepsilon}, y_{\varepsilon}\right)_{\varepsilon}$ converges to $\left(t_{0}, t_{0}, x_{0}, x_{0}\right)$ for some $\left(t_{0}, x_{0}\right) \in[0, T] \times \mathcal{O}$ and

$$
M_{\varepsilon}=\Phi\left(t_{\varepsilon}, s_{\varepsilon}, x_{\varepsilon}, y_{\varepsilon}\right) \rightarrow M^{*} \quad \text { and } \quad \phi_{\varepsilon}\left(t_{\varepsilon}, s_{\varepsilon}, x_{\varepsilon}, y_{\varepsilon}\right) \rightarrow 0
$$

In view of Ishii's Lemma (Lemma 4.4.2), there exist $M, N \in \mathbb{S}^{d}$ such that

$$
\begin{aligned}
& \left(\frac{1}{\varepsilon}\left(t_{\varepsilon}-s_{\varepsilon}\right), \frac{1}{\varepsilon}\left(x_{\varepsilon}-y_{\varepsilon}\right), M\right) \in \bar{P}^{2,+} U(t, x), \\
& \left(\frac{1}{\varepsilon}\left(t_{\varepsilon}-s_{\varepsilon}\right), \frac{1}{\varepsilon}\left(x_{\varepsilon}-y_{\varepsilon}\right), N\right) \in \bar{P}^{2,-} V_{\delta}(t, x) .
\end{aligned}
$$

From the viscosity sub-solution and super-solution characterization of $U$ and $V_{\delta}$ in terms of super-jets and sub-jets, we then have

$$
\begin{aligned}
& -\frac{1}{\varepsilon}\left(t_{\varepsilon}-s_{\varepsilon}\right)-H\left(t_{\varepsilon}, x_{\varepsilon}, U\left(t_{\varepsilon}, x_{\varepsilon}\right), \frac{1}{\varepsilon}\left(x_{\varepsilon}-y_{\varepsilon}\right), M\right) \leq 0, \\
& -\frac{1}{\varepsilon}\left(t_{\varepsilon}-s_{\varepsilon}\right)-H\left(s_{\varepsilon}, y_{\varepsilon}, V_{\delta}\left(s_{\varepsilon}, y_{\varepsilon}\right), \frac{1}{\varepsilon}\left(x_{\varepsilon}-y_{\varepsilon}\right), N\right) \geq 0 .
\end{aligned}
$$

By subtracting the two inequalities above, we get

$$
H\left(t_{\varepsilon}, x_{\varepsilon}, U\left(t_{\varepsilon}, x_{\varepsilon}\right), \frac{1}{\varepsilon}\left(x_{\varepsilon}-y_{\varepsilon}\right), M\right) \geq H\left(s_{\varepsilon}, y_{\varepsilon}, V_{\delta}\left(s_{\varepsilon}, y_{\varepsilon}\right), \frac{1}{\varepsilon}\left(x_{\varepsilon}-y_{\varepsilon}\right), N\right)
$$

Subtracting $H\left(t_{\varepsilon}, x_{\varepsilon}, V_{\delta}\left(s_{\varepsilon}, y_{\varepsilon}\right), \frac{1}{\varepsilon}\left(x_{\varepsilon}-y_{\varepsilon}\right), M\right)$ from both sides of the equation above, we get

$$
\begin{aligned}
& H\left(t_{\varepsilon}, x_{\varepsilon}, U\left(t_{\varepsilon}, x_{\varepsilon}\right), \frac{1}{\varepsilon}\left(x_{\varepsilon}-y_{\varepsilon}\right), M\right)-H\left(t_{\varepsilon}, x_{\varepsilon}, V_{\delta}\left(s_{\varepsilon}, y_{\varepsilon}\right), \frac{1}{\varepsilon}\left(x_{\varepsilon}-y_{\varepsilon}\right), M\right) \geq \\
& H\left(s_{\varepsilon}, y_{\varepsilon}, V_{\delta}\left(s_{\varepsilon}, y_{\varepsilon}\right), \frac{1}{\varepsilon}\left(x_{\varepsilon}-y_{\varepsilon}\right), N\right)-H\left(t_{\varepsilon}, x_{\varepsilon}, V_{\delta}\left(s_{\varepsilon}, y_{\varepsilon}\right), \frac{1}{\varepsilon}\left(x_{\varepsilon}-y_{\varepsilon}\right), M\right)
\end{aligned}
$$

Denote the left and right hand side of the inequality above by LHS and RHS. On the one hand, since $U\left(t_{\varepsilon}, x_{\varepsilon}\right)-V_{\delta}\left(s_{\varepsilon}, y_{\varepsilon}\right) \geq M^{*}$,

$$
\mathrm{LHS} \leq-\gamma\left(U\left(t_{\varepsilon}, x_{\varepsilon}\right)-V_{\delta}\left(s_{\varepsilon}, y_{\varepsilon}\right)\right) \leq-\gamma M^{*}
$$


On the other hand, applying inequality (4.4.5) to $C=\sigma_{X}\left(t_{\varepsilon}, x_{\varepsilon}, a\right)$ and $D=$ $\sigma_{X}\left(s_{\varepsilon}, y_{\varepsilon}, a\right)$, we get

$$
\begin{aligned}
I_{1}: & =\left|\frac{1}{2} \operatorname{Tr}\left[\sigma_{X} \sigma_{X}^{\top}\left(t_{\varepsilon}, x_{\varepsilon}, a\right) M\right]-\frac{1}{2} \operatorname{Tr}\left[\sigma_{X} \sigma_{X}^{\top}\left(s_{\varepsilon}, y_{\varepsilon}, a\right) N\right]\right| \\
& \left.\leq \frac{3}{2 \varepsilon} \operatorname{Tr}\left[\left(\sigma_{X}\left(t_{\varepsilon}, x_{\varepsilon}\right)-\sigma_{X}\left(s_{\varepsilon}, y_{\varepsilon}\right)\right)\left(\sigma_{X}\left(t_{\varepsilon}, x_{\varepsilon}\right)-\sigma_{X}\left(s_{\varepsilon}, y_{\varepsilon}\right)\right)^{\top}\right)\right] \\
& \leq \frac{1}{2 \varepsilon} O\left(\left|t_{\varepsilon}-s_{\varepsilon}\right|^{2}+\left|x_{\varepsilon}-y_{\varepsilon}\right|^{2}\right) \rightarrow 0 .
\end{aligned}
$$

In the last inequality, we use (4.3.42) and Lipschitz continuity of $\sigma_{X}$ (uniformly in a). Therefore,

$$
I_{1} \rightarrow 0 \text { as } \varepsilon \rightarrow 0, \text { uniformly in } a \in A \text {. }
$$

Similarly, from (4.3.42) and the Lipschitz continuity of $\mu_{X}$ (uniformly in $a$ )

$$
I_{2}:=\left|\frac{1}{\varepsilon} \mu_{X}^{\top}\left(t_{\varepsilon}, x_{\varepsilon}, a\right)\left(x_{\varepsilon}-y_{\varepsilon}\right)-\frac{1}{\varepsilon} \mu_{X}^{\top}\left(s_{\varepsilon}, y_{\varepsilon}, a\right)\left(x_{\varepsilon}-y_{\varepsilon}\right)\right| \rightarrow 0 \text { for all } a \in A
$$

From (4.3.42) and Lipschitz continuity of $\sigma_{X}$ (Assumption 4.2.1) and $\mu_{Y}^{\hat{u}}$ (Assumption 4.2.9), we get

$$
\begin{aligned}
I_{3}:= & \mid \mu_{Y}^{\hat{u}}\left(t_{\varepsilon}, x_{\varepsilon}, V_{\delta}\left(s_{\varepsilon}, y_{\varepsilon}\right), \sigma_{X}\left(t_{\varepsilon}, x_{\varepsilon}, a\right)\left(\frac{x_{\varepsilon}-y_{\varepsilon}}{\varepsilon}\right), a\right) \\
& -\mu_{Y}^{\hat{u}}\left(s_{\varepsilon}, y_{\varepsilon}, V_{\delta}\left(s_{\varepsilon}, y_{\varepsilon}\right), \sigma_{X}\left(s_{\varepsilon}, y_{\varepsilon}, a\right)\left(\frac{x_{\varepsilon}-y_{\varepsilon}}{\varepsilon}\right), a\right) \mid \\
\leq & \nu\left(\left|t_{\varepsilon}-s_{\varepsilon}\right|+\left|x_{\varepsilon}-y_{\varepsilon}\right|\right)+\frac{1}{2 \varepsilon} O\left(\left|t_{\varepsilon}-s_{\varepsilon}\right|^{2}+\left|x_{\varepsilon}-y_{\varepsilon}\right|^{2}\right) \rightarrow 0 \text { as } \varepsilon \rightarrow 0,
\end{aligned}
$$

where $\nu(z) \rightarrow 0$ as $z \rightarrow 0$. The first term in the last inequality above is the modulus of continuity of $\mu_{Y}^{\hat{u}}$ in the variables $(t, x)$ (uniformly in $a$ ) and the second term comes from similar arguments for $I_{1}$ and $I_{2}$. Therefore,

$$
I_{3} \rightarrow 0 \text {, uniformly in } a \in A \text {. }
$$

Then, (4.3.45), (4.3.46) and (4.3.47) imply that

$$
\mathrm{RHS} \rightarrow 0 \quad \text { as } \quad \varepsilon \rightarrow 0
$$

From (4.3.43), (4.3.44) and (4.3.48), we obtain a contradiction. 
Corollary 4.3.6. If $g$ is continuous and Assumptions 4.2.1, 4.2.6, 4.2.9, 4.2.10 and 4.2.11 hold, then $v$ is the unique bounded continuous viscosity solution of (4.2.4).

Proof. From 4.3.4, $v^{+}$(resp. $v^{-}$) is a bounded USC viscosity sub-solution (resp. LSC viscosity super-solution) to (4.2.4). Then, $v^{+}(T, x) \leq g(x) \leq v^{-}(T, x)$. This implies $v^{+} \leq v^{-}$on $\mathbb{D}$ from Proposition 4.3.5. Since $v^{+} \geq v \geq v^{-}$by definition, $v^{+}=v=v^{-}$. We have shown that $v$ is continuous and a bounded viscosity solution of (4.2.4).

To check the uniqueness, let $w$ be a bounded continuous viscosity solution of (4.2.4). Note that $w$ is a LSC viscosity super-solution and $v$ is an USC viscosity sub-solution of (4.2.4) . From Proposition 4.3.5, $v \leq w$ on $\mathbb{D}$. Similarly, $w \leq v$ on $\mathbb{D}$. This implies $w=v$ on $\mathbb{D}$.

From Theorem 4.3.4 and Corollary 4.3.6, we obtain dynamic programming principle as a byproduct.

Corollary 4.3.7 (Dynamic Programming Principle). Assume $g$ is continuous and Assumptions 4.2.1-4.2.11 hold. For any $(t, x) \in \mathbb{D}$, the following two statements hold:

1. (DPP 1.) For any $y>v(t, x)$, there exists $\mathfrak{u} \in \mathfrak{U}(t)$ such that for all $\alpha \in \mathcal{A}^{t}$ and $\theta \in \mathcal{T}_{t}$,

$$
Y_{t, x, y}^{\mathfrak{u}, \alpha}(\theta) \geq v\left(\theta, X_{t, x}^{\alpha}(\theta)\right)
$$

2. (DPP 2.) For any $y<v(t, x)$ and $\mathfrak{u} \in \mathfrak{U}(t)$, there exists $\alpha \in \mathcal{A}^{t}$ such that for all $\theta \in \mathcal{T}_{t}$

$$
\mathbb{P}\left(Y_{t, x, y}^{\mathfrak{u}, \alpha} \geq v\left(\theta, X_{t, x}^{\alpha}(\theta)\right)\right)<1
$$

Proof. DPP 1: If $y>v(t, x)=v^{+}(t, x)$ (due to Corollary 4.3.6), there exists $w \in \mathbb{U}^{+}$ such that $y>w(t, x)$. From the definition of stochastic super-solution, there exists 
$\mathfrak{u} \in \mathfrak{U}(t)$ such that

$$
Y_{t, x, y}^{\mathfrak{u}, \alpha}(\theta) \geq w\left(\theta, X_{t, x}^{\alpha}(\theta)\right) \geq v\left(\theta, X_{t, x}^{\alpha}(\theta)\right) \text { for all } \theta \in \mathcal{T}_{t} \text { and } \alpha \in \mathcal{A}^{t}
$$

DPP 2: If $y<v(t, x)=v^{-}(t, x)=\sup _{w \in \mathbb{U}^{-}} w(t, x)$ there exists $w \in \mathbb{U}^{-}$such that $y<w(t, x)$. From the definition of stochastic sub-solution, for any $\mathfrak{u} \in \mathfrak{U}(t)$, there exits $\alpha \in \mathcal{A}^{t}$ such that

$$
\mathbb{P}\left(Y_{t, x, y}^{\mathfrak{u}, \alpha}(\theta)<w\left(\theta, X_{t, x}^{\alpha}(\theta)\right)\right)>0 \text { for all } \theta \in \mathcal{T}_{t}
$$

Since $w\left(\theta, X_{t, x}^{\alpha}(\theta)\right) \leq v\left(\theta, X_{t, x}^{\alpha}(\theta)\right)$, the desired result holds.

\subsection{Appendix}

\subsubsection{Proof of Proposition 4.2.12}

We carry out the proof in two steps. First under Assumptions 4.2.6 and 4.2.9, we will show that there exists a classical solution to (4.2.4). Next, we will show that, if we additionally have Assumption 4.2.1, then every classical super-solution is a stochastic super-solution, which implies in particular that $\mathbb{U}^{+}$is not empty.

\section{Step 1. Existence of a classical super-solution to (4.2.4).}

1.A. In this step we will assume that $\mu_{Y}^{\hat{u}}$ is non-decreasing in its $y$-variable. Letting $\phi(t, x)=-e^{\lambda t}$, we have that

$$
\partial_{t} \phi(t, x)+H\left(t, x, \phi, D \phi, D^{2} \phi\right)=-\lambda e^{\lambda t}+\sup _{a \in A}\left\{-\mu_{Y}^{\hat{u}}(t, x, \phi(t, x), 0, a)\right\} .
$$

From the linear growth condition of $\mu_{Y}^{\hat{u}}$ in Assumption 4.2.9, we know there exists an $L>0$, such that $-\mu_{Y}^{\hat{u}}(t, x, \phi(t, x), 0, a) \leq L(1+|\phi(t, x)|)=L\left(1+e^{\lambda t}\right)$. Therefore, from (4.4.1),

$$
\partial_{t} \phi(t, x)+H\left(t, x, \phi, D \phi, D^{2} \phi\right) \leq-\lambda e^{\lambda t}+L\left(1+e^{\lambda t}\right) \leq 0 \text { on } \mathbb{D}_{i} \text { for } \lambda>2 L \text {. }
$$


Fix $\lambda>2 L$ and choose $N_{2}$ such that $-e^{\lambda T}+N_{2} \geq\|g\|_{\infty}$. Then $\phi^{\prime}(T, x)=\phi(T, x)+$ $N_{2} \geq g(x)$ for all $x \in \mathbb{R}^{d}$. From the assumption that $\mu_{Y}^{\hat{u}}$ is non-decreasing in its $y$-variable, it holds that

$$
\partial_{t} \phi^{\prime}(t, x)+H\left(t, x, \phi^{\prime}, D \phi^{\prime}, D^{2} \phi^{\prime}\right) \leq 0 \text { on } \mathbb{D}_{i}
$$

Therefore, $\phi^{\prime}$ is a classical super-solution.

1.B. We now turn to showing the same result for more general $\mu_{Y}^{\hat{u}}$. This follows the same reparameterization argument outlined in Step 1.2-B in the proof of the main theorem.

Step 2. Classical super-solutions are stochastic super-solutions. Let $w$ be a classical super-solution. Fix $(t, x, y) \in \mathbb{D}_{i} \times \mathbb{R}$ and $\left\{\tau^{\alpha}\right\} \in \mathbb{T}_{t}$. Let $\bar{Y}$ be the unique strong solution (which is thanks to Assumption 4.2.9) of the equation

$$
\begin{aligned}
\bar{Y}(l)= & Y_{t, x, y}^{\mathfrak{u}, \alpha}\left(\tau^{\alpha}\right)+\int_{\tau^{\alpha}}^{\tau^{\alpha} \vee l} \mu_{Y}^{\hat{u}}\left(s, X_{t, x}^{\alpha}(s), \bar{Y}(s), \sigma_{X}\left(s, X_{t, x}^{\alpha}(s), \alpha_{s}\right) D w\left(s, X_{t, x}^{\alpha}(s)\right), \alpha_{s}\right) d s \\
& +\int_{\tau^{\alpha}}^{\tau^{\alpha} \vee l} \sigma_{X}\left(s, X_{t, x}^{\alpha}(s), \alpha_{s}\right) D w\left(s, X_{t, x}^{\alpha}(s)\right) d W_{s}, \quad l \geq \tau^{\alpha},
\end{aligned}
$$

for any $\mathfrak{u} \in \mathfrak{U}(t)$ and $\alpha \in \mathcal{A}^{t}$ and set $\bar{Y}(s)=Y_{t, x, y}^{\mathfrak{u}, \alpha}(s)$ for $s<\tau^{\alpha}$. We will set $\tilde{\mathfrak{u}}$ to be

$$
\tilde{\mathfrak{u}}:=\tilde{\mathfrak{u}}[\alpha](s)=\hat{u}\left(s, X_{t, x}^{\alpha}(s), \bar{Y}(s), \sigma_{X}\left(s, X_{t, x}^{\alpha}(s), \alpha_{s}\right) D w\left(s, X_{t, x}^{\alpha}(s)\right), \alpha_{s}\right) .
$$

It is not difficult to check that $\tilde{\mathfrak{u}} \in \mathfrak{U}\left(t,\left\{\tau^{\alpha}\right\}\right)$. We will show that for any $\mathfrak{u} \in \mathfrak{U}(t)$, $\alpha \in \mathcal{A}^{t}$ and each stopping time $\rho \in \mathcal{T}_{t}, \tau^{\alpha} \leq \rho \leq T$ with the simplifying notation $X:=X_{t, x}^{\alpha}, Y:=Y_{t, x, y}^{\mathfrak{u} \otimes_{\tau}^{\alpha} \tilde{\mathfrak{u}}[\alpha], \alpha}$, we have

$$
Y(\rho) \geq w(\rho, X(\rho)) \mathbb{P}-\text { a.s. } \quad \text { on } \quad\left\{Y\left(\tau^{\alpha}\right)>w\left(\tau^{\alpha}, X\left(\tau^{\alpha}\right)\right)\right\}
$$

Note that $\bar{Y}=Y_{t, x, y}^{\mathfrak{u} \otimes_{\tau} \alpha \tilde{u}[\alpha], \alpha}$ for $s \geq \tau^{\alpha}$. We will carry out the rest of the proof in two steps.

2.A. In this step we will assume that $\mu_{Y}^{\hat{u}}$ is non-decreasing in its $y$-variable. Let

$$
A=\left\{Y\left(\tau^{\alpha}\right)>w\left(\tau^{\alpha}, X\left(\tau^{\alpha}\right)\right)\right\}, Z(s)=w(s, X(s)), \quad \Gamma(s)=(Z(s)-Y(s)) \mathbb{1}_{A} .
$$


Therefore,

$$
\Gamma(s)=\mathbb{1}_{A} \int_{\tau^{\alpha}}^{s \vee \tau^{\alpha}}\left(\xi(u)-\gamma^{\prime}(u)\right) d u
$$

where

$$
\begin{aligned}
\gamma^{\prime}(s): & =\mu_{Y}^{\hat{u}}\left(s, X(s), w(s, X(s)), \sigma_{X}\left(s, X(s), \alpha_{s}\right) D w(s, X(s)), \alpha_{s}\right)-\partial_{t} w(s, X(s)) \\
& -\mu_{X}^{\top}\left(s, X(s), \alpha_{s}\right) D w(s, X(s))-\frac{1}{2} \operatorname{Tr}\left[\sigma_{X} \sigma_{X}^{\top}\left(s, X(s), \alpha_{s}\right) D^{2} w(s, X(s))\right]
\end{aligned}
$$

and

$$
\begin{aligned}
\xi(s): & =\mu_{Y}^{\hat{u}}\left(s, X(s), Z(s), \sigma_{X}\left(s, X(s), \alpha_{s}\right) D w(s, X(s)), \alpha_{s}\right) \\
& -\mu_{Y}^{\hat{u}}\left(s, X(s), Y(s), \sigma_{X}\left(s, X(s), \alpha_{s}\right) D w(s, X(s)), \alpha_{s}\right) .
\end{aligned}
$$

Since $w$ is a classical super-solution, $\gamma^{\prime} \geq 0$. Then from (4.4.2), it follows that

$$
\Gamma(s) \leq \mathbb{1}_{A} \int_{\tau^{\alpha}}^{s} \xi(u) d u \quad \text { and } \quad \Gamma^{+}(s) \leq \mathbb{1}_{A} \int_{\tau^{\alpha}}^{s} \xi^{+}(u) d u, \text { for } s \geq \tau^{\alpha}
$$

From the Lipschitz continuity of $\mu_{Y}^{\hat{u}}$ in $y$-variable in Assumption 4.2.9,

$$
\Gamma^{+}(s) \leq \mathbb{1}_{A} \int_{\tau^{\alpha}}^{s} \xi^{+}(u) d u \leq \int_{\tau^{\alpha}}^{s} L \Gamma^{+}(u) d u \text { for } s \geq \tau^{\alpha}
$$

In the equation above, we also use the assumption that $\mu_{Y}^{\hat{u}}$ is non-decreasing in its $y$-variable to obtain the second inequality. Since $\mathbb{E} \Gamma^{+}\left(\tau^{\alpha}\right)=0$, an application of Gronwall's Inequality implies that $\mathbb{E} \Gamma^{+}(\rho) \leq 0$.

2.B. Now let us turn to showing same result for more general $\mu_{Y}^{\hat{u}}$. However, this again follows the same reparameterization argument outlined in Step 1.2-B in the proof of the main theorem.

\subsubsection{Proof of Proposition 4.2.17}

Take $w(t, x)=m$ for any $(t, x) \in \mathbb{D}$, where the constant $m$ is a lower bound of $g$. For any given $\mathfrak{u} \in \mathfrak{U}(t), \alpha \in \mathcal{A}^{t}$, choose any $\tilde{\alpha} \in \mathcal{A}^{t}$. Let $B \subset\left\{Y\left(\tau^{\alpha}\right)<w\left(\tau, X\left(\tau^{\alpha}\right)\right)\right\}$ 
and $\mathbb{P}(B)>0$. Set

$$
\theta_{s}:=\left\{\begin{array}{l}
0 \quad \text { if } \sigma_{Y}\left(s, X(s), Y(s), \mathfrak{u}\left[\alpha \otimes_{\tau^{\alpha}} \tilde{\alpha}\right]_{s},\left[\alpha \otimes_{\tau^{\alpha}} \tilde{\alpha}\right]_{s}\right) \neq 0 \\
\frac{\mu_{Y} \sigma_{Y}}{\left\|\sigma_{Y}\right\|^{2}}\left(s, X(s), Y(s), \mathfrak{u}\left[\alpha \otimes_{\tau^{\alpha}} \tilde{\alpha}\right]_{s},\left[\alpha \otimes_{\tau^{\alpha}} \tilde{\alpha}\right]_{s}\right), \text { otherwise. }
\end{array}\right.
$$

Therefore, $\theta_{s}$ satisfies the Novikov's condition due to Assumption 4.2 .16 and $\widetilde{W}(s)=$ $W(s)-\int_{0}^{s} \theta_{u} d u$ is a Brownian motion under the probability measure $\mathbb{Q}$, where

$$
\mathbb{Q}(A)=\mathbb{E}_{\mathbb{P}}\left(Z_{T} \mathbb{1}_{A}\right) \text { for all } A \in \mathcal{F}, \text { and } Z_{s}:=\exp \left(\int_{0}^{s} \theta_{u} d W_{u}-\frac{1}{2} \int_{0}^{s}\left\|\theta_{u}\right\|^{2} d u\right) .
$$

$Z_{T} \in \mathbb{L}^{q}(\mathbb{P})$ for any $q \geq 1$ since $\theta$ is a bounded. From Assumption 4.2 .13 and assumption that $\sigma_{Y}$ is invertible in its $u$-variable (Assumption 4.2.6), it follows that $\sigma_{Y}(t, x, y, u, a)=0$ implies $\mu_{Y}(t, x, y, u, a)=0$. Therefore under $\mathbb{Q}$

$$
d Y(s)=\sigma_{Y}\left(s, X(s), Y(s), \mathfrak{u}[\tilde{\alpha}]_{s}, \tilde{\alpha}_{s}\right) d \widetilde{W}_{s} \quad \text { for } \quad s \geq \tau^{\alpha}
$$

where $Y:=Y_{t, x, y}^{\mathfrak{u}, \alpha \otimes_{\tau} \alpha \tilde{\alpha}}$. We will show that the $\mathbb{Q}$-local martingale $Y$ is actually a Q-martingale. Assumption 4.2.1 implies that

$$
\mathbb{E}_{\mathbb{P}}\left[\sup _{0 \leq s \leq T}|Y(s)|^{2}\right]<\infty
$$

See e.g. Theorem 1.3.5 in [26] or Theorem 2.2 in [34]. As a result, an application of Hölder's inequality yields that

$$
\mathbb{E}_{\mathbb{Q}}\left[\sup _{0 \leq s \leq T}|Y(s)|\right] \leq \mathbb{E}_{\mathbb{P}}\left[\sup _{0 \leq s \leq T}|Y(s)|^{2}\right] \mathbb{E}_{\mathbb{P}}\left[Z_{T}^{2}\right]<\infty .
$$

From (4.4.4), $Y$ is a martingale on $\left[\tau^{\alpha}, T\right]$ under $\mathbb{Q}$. Moreover, since $\mathbb{Q}$ is equivalent to $\mathbb{P}, \mathbb{Q}(B)>0$. As a result, for any $\rho \geq \tau^{\alpha}$,

$$
Y(\rho) \leq Y\left(\tau^{\alpha}\right)<m \text { on some } \mathcal{F}_{\tau^{\alpha}}^{t} \text {-measurable set } H \subset B \text { with } \mathbb{Q}(H)>0 \text {. }
$$

This implies $\mathbb{Q}(Y(\rho)<m \mid B)>0$ and $\mathbb{P}(Y(\rho)<m \mid B)>0$ by the fact $\mathbb{P} \sim \mathbb{Q}$. Therefore, $w(t, x)=m$ is a stochastic sub-solution. 


\subsubsection{Some well-known results from the theory of viscosity solutions}

In this subsection, we introduce an alternative definition of viscosity solutions and Ishii's Lemma following [26]. First, we define the second order super-jet of an USC function $U$ at a point $(\bar{t}, \bar{x}) \in[0, T) \times \mathbb{R}^{d}$ as the set of elements $(\bar{q}, \bar{p}, \bar{M}) \in \mathbb{R} \times \mathbb{R}^{d} \times \mathbb{S}^{d}$ satisfying

$U(t, x) \leq U(\bar{t}, \bar{x})+\bar{q}(t-\bar{t})+\bar{p} \cdot(x-\bar{x})+\frac{1}{2} M(x-\bar{x}) \cdot(x-\bar{x})+o\left(|t-\bar{t}|+|x-\bar{x}|^{2}\right)$.

This set is denoted by $P^{2,+} U(\bar{t}, \bar{x})$. Similarly, $P^{2,-} V(\bar{t}, \bar{x})$, the second-order sub-jet of a LSC function $\mathrm{V}$ at the point $(\bar{t}, \bar{x}) \in[0, T) \times \mathbb{R}^{d}$ is defined as the set of elements $(\bar{q}, \bar{p}, \bar{M}) \in \mathbb{R} \times \mathbb{R}^{d} \times \mathbb{S}^{d}$ satisfying

$V(t, x) \geq V(\bar{t}, \bar{x})+\bar{q}(t-\bar{t})+\bar{p} \cdot(x-\bar{x})+\frac{1}{2} M(x-\bar{x}) \cdot(x-\bar{x})+o\left(|t-\bar{t}|+|x-\bar{x}|^{2}\right)$.

For technical reasons related to Ishii's lemma, we also need to consider the limiting super-jets and sub-jets. More precisely, we define $\bar{P}^{2,+} U(t, x)$ as the set of elements $(q, p, M) \in \mathbb{R} \times \mathbb{R}^{d} \times \mathbb{S}^{d}$ for which there exists a sequence $\left(t_{\varepsilon}, x_{\varepsilon}, q_{\varepsilon}, p_{\varepsilon}, M_{\varepsilon}\right)_{\varepsilon}$ satisfying $\left(q_{\varepsilon}, p_{\varepsilon}, M_{\varepsilon}\right) \in P^{2,+} U\left(t_{\varepsilon}, x_{\varepsilon}\right)$ and $\left(t_{\varepsilon}, x_{\varepsilon}, U\left(t_{\varepsilon}, x_{\varepsilon}\right), q_{\varepsilon}, p_{\varepsilon}, M_{\varepsilon}\right) \rightarrow(t, x, U(t, x), q, p, M)$. The set $\bar{P}^{2,-} V(t, x)$ is defined similarly. Now we state the alternative definition of viscosity solutions to (4.2.4).

Lemma 4.4.1. A USC (resp. LSC) function $w$ on $\mathbb{D}_{i}$ is a viscosity sub-solution (resp. super-solution) to (4.2.4) if and only if for all $(t, x) \in \mathbb{D}_{i}$, and all $(q, p, M) \in$ $\bar{P}^{2,+} w(t, x)\left(\operatorname{resp} \cdot \bar{P}^{2,-} w(t, x)\right)$,

$$
-q-H(t, x, w(t, x), p, M) \leq(r e s p . \geq) 0
$$

Finally, we state Ishii's lemma used in [26] without proof and refer the reader to Theorem 8.3 in [18]. 
Lemma 4.4.2 (Ishii's Lemma). Let $U$ (resp. $V$ ) be an USC (resp. LSC) function on $\mathbb{D}_{i}, \varphi \in C^{1,1,2,2}\left([0, T)^{2} \times \mathbb{R}^{d} \times \mathbb{R}^{d}\right)$ and $\left(t_{0}, s_{0}, x_{0}, y_{0}\right) \in[0, T)^{2} \times \mathbb{R}^{d} \times \mathbb{R}^{d}$ be a local maximum of $U(t, x)-V(s, y)-\varphi(t, s, x, y)$. Then, for all $\eta>0$, there exist $M, N \in \mathbb{S}^{d}$ satisfying

$$
\begin{gathered}
\left(\partial_{t} \varphi\left(t_{0}, s_{0}, x_{0}, y_{0}\right), D_{x} \varphi\left(t_{0}, s_{0}, x_{0}, y_{0}\right), M\right) \in \bar{P}^{2,+} U(t, x), \\
\left(-\varphi_{s}\left(t_{0}, s_{0}, x_{0}, y_{0}\right),-D_{y} \varphi\left(t_{0}, s_{0}, x_{0}, y_{0}\right), N\right) \in \bar{P}^{2,-} V(t, x),
\end{gathered}
$$

and

$$
\left(\begin{array}{cc}
M & 0 \\
0 & -N
\end{array}\right) \leq D_{x, y}^{2} \varphi\left(t_{0}, s_{0}, x_{0}, y_{0}\right)+\eta\left(D_{x, y}^{2} \varphi\left(t_{0}, s_{0}, x_{0}, y_{0}\right)\right)^{2} .
$$

Remark 4.4.3. From Remark 4.4.9 in [26], by choosing $\varphi_{\varepsilon}(t, s, x, y):=\frac{1}{2 \varepsilon}\left(|t-s|^{2}+\right.$ $\left.|x-y|^{2}\right)$ and $\eta=\varepsilon$, for any $d \times n$ matrices $C, D$, we get

$$
\operatorname{Tr}\left(C C^{\top} M-D D^{\top} N\right) \leq \frac{3}{\varepsilon} \operatorname{Tr}\left((C-D)(C-D)^{\top}\right)
$$




\section{CHAPTER V}

\section{Stochastic Perron for Stochastic Target Games with a Stopper}

\subsection{Outline of this chapter}

As mentioned in the introduction, in this chapter we investigate two types of stochastic target games with a stopper under the framework of Chapter IV. The two types of stochastic target problems can be interpreted as the super-hedging and sub-hedging problem, respectively. In Section 5.2, both problems and their associated HJB equations are introduced. In Sections 5.3 and 5.4, we prove the viscosity properties and verify that the value function is the unique viscosity solution to its associated HJB equation for the super-hedging and sub-hedging problem, respectively. In Section 5.5, we compare the two value functions and prove that they coincide when the control set of Nature is a singleton.

\subsection{The setup}

Let $\Omega$ be the space of continuous functions $\omega:[0, T] \rightarrow \mathbb{R}^{d}, \mathbb{P}$ be the Wiener measure on $\Omega$ and $W$ be the canonical process on $\Omega$, i.e. $W_{t}(\omega)=\omega_{t}$. For $t \in[0, T]$, let $\mathbb{F}^{t}=\left(\mathcal{F}_{s}^{t}\right)_{0 \leq s \leq T}$ be the augmented filtration generated by $\left(W_{s}-W_{t}\right)_{s \geq t}$. By convention, $\mathcal{F}_{s}^{t}:=\mathcal{F}_{t}^{t}$ for $s<t$. Denote by $\mathcal{U}^{t}\left(\right.$ resp. $\left.\mathcal{A}^{t}\right)$ the collection of all $\mathbb{F}^{t}$

predictable processes in $\mathbb{L}^{2}\left(\mathbb{P} \otimes \lambda_{L}\right)$ with values in a given Borel subset $U$ (resp. 
bounded set $A$ ) of $\mathbb{R}^{k}$ (resp. $\mathbb{R}^{l}$ ), where $\lambda_{L}$ is the Lebesgue measure on $\mathbb{R}$. Let

$$
\mathbb{D}:=[0, T] \times \mathbb{R}^{d}, \quad \mathbb{D}_{i}:=[0, T) \times \mathbb{R}^{d}, \quad \mathbb{D}_{T}:=\{T\} \times \mathbb{R}^{d} .
$$

Given $(t, x, y) \in \mathbb{D} \times \mathbb{R}$ and $(u, \alpha) \in \mathcal{U}^{t} \times \mathcal{A}^{t}$, the state processes are driven by the stochastic differential equations (SDEs)

$$
\begin{aligned}
& d X(s)=\mu_{X}\left(s, X(s), \alpha_{s}\right) d s+\sigma_{X}\left(s, X(s), \alpha_{s}\right) d W_{s}, \\
& d Y(s)=\mu_{Y}\left(s, X(s), Y(s), u_{s}, \alpha_{s}\right) d s+\sigma_{Y}\left(s, X(s), Y(s), u_{s}, \alpha_{s}\right) d W_{s},
\end{aligned}
$$

with initial data $(X(t), Y(t))=(x, y)$.

Assumption 5.2.1. $\mu_{X}, \mu_{Y}, \sigma_{X}$ and $\sigma_{Y}$ are continuous in all variables and take values in $\mathbb{R}^{d}, \mathbb{R}, \mathbb{R}^{d}$ and $\mathbb{M}^{d}$, respectively. There exists $K>0$ such that for all $(t, x, y),\left(t^{\prime}, x^{\prime}, y^{\prime}\right) \in \mathbb{D} \times \mathbb{R}, u \in U$ and $a \in A$,

$$
\begin{gathered}
\left|\mu_{X}(t, x, a)-\mu_{X}\left(t^{\prime}, x^{\prime}, a\right)\right|+\left|\sigma_{X}(t, x, a)-\sigma_{X}\left(t^{\prime}, x^{\prime}, a\right)\right| \leq K\left(\left|t-t^{\prime}\right|+\left|x-x^{\prime}\right|\right), \\
\left|\mu_{X}(t, x, a)\right|+\left|\sigma_{X}(t, x, a)\right| \leq K, \\
\left|\mu_{Y}(t, x, y, u, a)-\mu_{Y}\left(t, x, y^{\prime}, u, a\right)\right|+\left|\sigma_{Y}(t, x, y, u, a)-\sigma_{Y}\left(t, x, y^{\prime}, u, a\right)\right| \leq K\left|y-y^{\prime}\right|, \\
\left|\mu_{Y}(t, x, y, u, a)\right|+\left|\sigma_{Y}(t, x, y, u, a)\right| \leq K(1+|u|+|y|) .
\end{gathered}
$$

This assumption ensures that the stochastic differential equations given in (5.2.1) are well-posed. Denote the solutions to (5.2.1) by $\left(X_{t, x}^{\alpha}, Y_{t, x, y}^{u, \alpha}\right)$. Let $\mathcal{T}_{t}$ be the collection of all $\mathbb{F}^{t}$-stopping times valued in $[t, T]$.

Definition 5.2.2. A map $\mathfrak{u}: \mathcal{A}^{t} \rightarrow \mathcal{U}^{t}, \alpha \mapsto \mathfrak{u}[\alpha]$ is a $t$-admissible strategy if it is non-anticipating in the sense that

$$
\left\{\omega \in \Omega:\left.\alpha(\omega)\right|_{[t, s]}=\left.\alpha^{\prime}(\omega)\right|_{[t, s]}\right\} \subset\left\{\omega \in \Omega:\left.\mathfrak{u}[\alpha](\omega)\right|_{[t, s]}=\left.\mathfrak{u}\left[\alpha^{\prime}\right](\omega)\right|_{[t, s]}\right\} \text {-a.s. }
$$

for all $s \in[t, T]$ and $\alpha, \alpha^{\prime} \in \mathcal{A}^{t}$, where $\left.\right|_{[t, s]}$ indicates the restriction to the interval $[t, s]$. We denote by $\mathfrak{U}(t)$ the collection of all $t$-admissible strategies. We write $Y_{t, x, y}^{\mathfrak{u}, \alpha}$ for $Y_{t, x, y}^{\mathfrak{u}[\alpha], \alpha}$ for any $\mathfrak{u} \in \mathfrak{U}(t)$. 
Definition 5.2.3. A map $\rho: \mathcal{A}^{t} \rightarrow \mathcal{T}_{t}, \alpha \mapsto \boldsymbol{\rho}[\alpha]$ is a $t$-admissible stopping strategy if

$$
\begin{gathered}
\left\{\omega \in \Omega:\left.\alpha(\omega)\right|_{[t, s]}=\left.\alpha^{\prime}(\omega)\right|_{[t, s]}\right\} \subset \\
\left\{\omega \in \Omega: t \leq \boldsymbol{\rho}[\alpha](\omega)=\boldsymbol{\rho}\left(\alpha^{\prime}\right)(\omega) \leq s\right\} \cup\left\{\omega \in \Omega, s<\min \left\{\boldsymbol{\rho}[\alpha](\omega), \boldsymbol{\rho}\left(\alpha^{\prime}\right)(\omega)\right\}\right\}
\end{gathered}
$$

for all $s \in[t, T]$ and $\alpha, \alpha^{\prime} \in \mathcal{A}^{t}$. We denote by $\mathbf{T}(t)$ the collection of all $t$-admissible stopping strategies;

With the definitions above, we can rigorously define the two value functions, which can be interpreted as the super-hedging and sub-hedging price of American options with model uncertainty, respectively. ${ }^{1}$

$$
\begin{gathered}
V_{s}(t, x):=\inf \left\{y \in \mathbb{R}: \exists \mathfrak{u} \in \mathfrak{U}(t) \text { s.t. } Y_{t, x, y}^{\mathfrak{u}, \alpha}(\rho) \geq g\left(X_{t, x}^{\alpha}(\rho)\right) \forall \alpha \in \mathcal{A}^{t}, \forall \rho \in \mathcal{T}_{t}\right\}, \\
V_{b}(t, x):=\sup \left\{y \in \mathbb{R}: \exists \mathfrak{u} \in \mathfrak{U}(t), \boldsymbol{\rho} \in \mathbf{T}(t) \text { s.t. } Y_{t, x, y}^{\mathfrak{u}, \alpha}(\boldsymbol{\rho}[\alpha]) \leq g\left(X_{t, x}^{\alpha}(\boldsymbol{\rho}[\alpha])\right) \forall \alpha \in \mathcal{A}^{t}\right\},
\end{gathered}
$$

where $g: \mathbb{R}^{d} \rightarrow \mathbb{R}$ is a bounded continuous function. From the above definitions, it is unclear if $V_{b}$ compare with $V_{s}$, although we expect that $V_{s}$ is larger. We will prove that this is indeed the case in Section 5.5.

To identify appropriate definitions of stochastic semi-solutions in Section 5.3 and 5.4, we need the following definitions.

Definition 5.2.4 (Non-anticipating family of stopping times). Let $\left\{\tau^{\alpha}\right\}_{\alpha \in \mathcal{A}^{t}} \subset \mathcal{T}_{t}$ be a family of stopping times. This family is $t$-non-anticipating if

$$
\begin{gathered}
\left\{\omega \in \Omega:\left.\alpha(\omega)\right|_{[t, s]}=\left.\alpha^{\prime}(\omega)\right|_{[t, s]}\right\} \subset \\
\left\{\omega \in \Omega: t \leq \tau^{\alpha}(\omega)=\tau^{\alpha^{\prime}}(\omega) \leq s\right\} \cup\left\{\omega \in \Omega: s<\min \left\{\tau^{\alpha}(\omega), \tau^{\alpha^{\prime}}(\omega)\right\}\right\} \text {-a.s. }
\end{gathered}
$$

Denote the set of $t$-non-anticipating families of stopping times by $\mathbb{T}_{t}$.

\footnotetext{
${ }^{1}$ We will use "the super-hedging problem" and "the sub-hedging problem" to refer to the two problems in this chapter, when there is no ambiguity.
} 
Remark 5.2.5. For $\boldsymbol{\rho} \in \mathbf{T}(t),\{\boldsymbol{\rho}[\alpha]\}_{\alpha \in \mathcal{A}^{t}} \in \mathbb{T}_{t}$ by definition. Throughout this chapter, $\left\{\tau^{\alpha}\right\}$ will be used to represent $t$-non-anticipating family of stopping times $\left\{\tau^{\alpha}\right\}_{\alpha \in \mathcal{A}^{t}}$ for short, when $t$ is fixed and there is no ambiguity.

Definition 5.2.6 (Strategies starting at a non-anticipating family of stopping times). Fix $t$ and let $\left\{\tau^{\alpha}\right\} \in \mathbb{T}_{t}$. We say that a map $\mathfrak{u}: \mathcal{A}^{t} \rightarrow \mathcal{U}^{t}, \alpha \mapsto \mathfrak{u}[\alpha]$ is a $\left(t,\left\{\tau^{\alpha}\right\}\right)$ admissible strategy if it is non-anticipating in the sense that

$$
\begin{gathered}
\left\{\omega \in \Omega:\left.\alpha(\omega)\right|_{[t, s]}=\left.\alpha^{\prime}(\omega)\right|_{[t, s]}\right\} \subset\left\{\omega \in \Omega: s<\min \left\{\tau^{\alpha}(\omega), \tau^{\alpha^{\prime}}(\omega)\right\}\right\} \cup \\
\left\{\omega \in \Omega: t \leq \tau^{\alpha}(\omega)=\tau^{\alpha^{\prime}}(\omega) \leq s,\left.\mathfrak{u}[\alpha](\omega)\right|_{\left[\tau^{\alpha}(\omega), s\right]}=\left.\mathfrak{u}\left[\alpha^{\prime}\right](\omega)\right|_{\left[\tau^{\alpha^{\prime}}(\omega), s\right]}\right\} \text {-a.s. }
\end{gathered}
$$

for all $s \in[t, T]$ and $\alpha, \alpha^{\prime} \in \mathcal{A}^{t}$, denoted by $\mathfrak{u} \in \mathfrak{U}\left(t,\left\{\tau^{\alpha}\right\}\right)$.

Remark 5.2.7. The definitions for $\mathfrak{U}\left(t,\left\{\tau^{\alpha}\right\}\right)$ and $\mathfrak{U}(t)$ are consistent. In fact, if we set $\tau^{\alpha}=t$ for all $\alpha \in \mathcal{A}^{t}$, then the two definitions coincide.

Definition 5.2.8 (Stopping strategies after a non-anticipating family of stopping times). For $t \in[0, T]$ and $\left\{\tau^{\alpha}\right\} \in \mathbb{T}_{t}$, a map $\boldsymbol{\rho}: \mathcal{A}^{t} \rightarrow \mathcal{T}_{t}, \alpha \mapsto \boldsymbol{\rho}[\alpha]$ is a $\left(t,\left\{\tau^{\alpha}\right\}\right)$ stopping strategy if $\boldsymbol{\rho}[\alpha] \geq \tau^{\alpha}$ for all $\alpha \in \mathcal{A}^{t}$ and

$$
\begin{gathered}
\left\{\omega \in \Omega:\left.\alpha(\omega)\right|_{[t, s]}=\left.\alpha^{\prime}(\omega)\right|_{[t, s]}\right\} \subset\left\{\omega \in \Omega, s<\min \left\{\boldsymbol{\rho}[\alpha](\omega), \boldsymbol{\rho}\left(\alpha^{\prime}\right)(\omega)\right\}\right\} \\
\cup\left\{\omega \in \Omega: \tau^{\alpha}(\omega)=\tau^{\alpha^{\prime}}(\omega) \leq \boldsymbol{\rho}[\alpha](\omega)=\boldsymbol{\rho}\left(\alpha^{\prime}\right)(\omega) \leq s\right\}
\end{gathered}
$$

for all $s \in[t, T]$ and $\alpha, \alpha^{\prime} \in \mathcal{A}^{t}$. The set of all such stopping strategies is denoted by $\mathbf{T}\left(t,\left\{\tau^{\alpha}\right\}\right)$.

Remark 5.2.9. The definitions of $\mathbf{T}\left(t,\left\{\tau^{\alpha}\right\}\right)$ and $\mathbf{T}(t)$ are consistent. In fact, if we set $\tau^{\alpha}=t$ for all $\alpha \in \mathcal{A}^{t}$, then the two definitions coincide. It also holds that $\mathbf{T}\left(t,\left\{\tau^{\alpha}\right\}\right) \subset \mathbf{T}(t)$ by definition for any $\left\{\tau^{\alpha}\right\} \in \mathbb{T}_{t}$.

Definition 5.2.10 (Concatenation). For $\alpha_{1}, \alpha_{2} \in \mathcal{A}^{t}$ and $\tau \in \mathcal{T}_{t}$. The concatenation of $\alpha_{1}, \alpha_{2}$ is defined as follows:

$$
\alpha_{1} \otimes_{\tau} \alpha_{2}:=\alpha_{1} \mathbb{1}_{[t, \tau)}+\alpha_{2} \mathbb{1}_{[\tau, T]}
$$


The concatenation of elements in $\mathcal{U}^{t}$ is defined in the same way. The following lemma discusses about the admissibility of the pasted strategy at a non-anticipating family of stopping times; We refer the readers to Lemma 4.2 .5 for its proof.

Lemma 5.2.11. For $t \in[0, T]$ and $\left\{\tau^{\alpha}\right\} \in \mathbb{T}_{t}$, if $\mathfrak{u} \in \mathfrak{U}(t)$ and $\tilde{\mathfrak{u}} \in \mathfrak{U}\left(t,\left\{\tau^{\alpha}\right\}\right)$, then $\mathfrak{u}_{*} \in \mathfrak{U}(t)$, where

$$
\mathfrak{u}_{*}[\alpha]:=\mathfrak{u} \otimes_{\tau^{\alpha}} \tilde{\mathfrak{u}}[\alpha]:=\mathfrak{u}[\alpha] \otimes_{\tau^{\alpha}} \tilde{\mathfrak{u}}[\alpha]
$$

For $(t, x, y, z, a) \in \mathbb{D} \times \mathbb{R} \times \mathbb{R}^{d} \times A$, define

$$
N(t, x, y, z, a):=\left\{u \in U: \sigma_{Y}(t, x, y, u, a)=z\right\}
$$

Assumption 5.2.12. There exists a measurable map $\hat{u}: \mathbb{D} \times \mathbb{R} \times \mathbb{R}^{d} \times A \rightarrow U$ such that $N=\{\hat{u}\}$. Moreover, the map $\hat{u}(\cdot, a)$ is continuous for each $a \in A$.

For $(t, x, y, p, z, M, a) \in \mathbb{D} \times \mathbb{R} \times \mathbb{R}^{d} \times \mathbb{R}^{d} \times \mathbb{M}^{d} \times A$, define

$$
\begin{gathered}
\mu_{Y}^{\hat{u}}(t, x, y, z, a):=\mu_{Y}(t, x, y, \hat{u}(t, x, y, z, a), a), \\
L^{a}(t, x, y, p, M):=\mu_{Y}^{\hat{u}}\left(t, x, y, \sigma_{X}(t, x, a) p, a\right)-\mu_{X}(t, x, a)^{\top} p-\frac{1}{2} \operatorname{Tr}\left[\sigma_{X} \sigma_{X}^{\top}(t, x, a) M\right], \\
L^{u, a}(t, x, y, p, M):=\mu_{Y}(t, x, y, u, a)-\mu_{X}(t, x, a)^{\top} p-\frac{1}{2} \operatorname{Tr}\left[\sigma_{X} \sigma_{X}^{\top}(t, x, a) M\right] . \\
H(t, x, y, p, M):=\inf _{a \in A} L^{a}(t, x, y, p, M), F(t, x, y, p, M):=\sup _{a \in A} L^{a}(t, x, y, p, M) .
\end{gathered}
$$

Assumption 5.2.13. The $\operatorname{map}(t, x, y, z) \in \mathbb{D} \times \mathbb{R} \times \mathbb{R}^{d} \mapsto \mu_{Y}^{\hat{u}}(t, x, y, z, a)$ is Lipschitz continuous, uniformly in $a \in A$. Moreover, $(y, z) \in \mathbb{R} \times \mathbb{R}^{d} \mapsto \mu_{Y}^{\hat{u}}(t, x, y, z, a)$ has linear growth, uniformly in $(t, x, a) \in \mathbb{D} \times A$.

\section{Assumption 5.2.14.}

$$
\sup _{u \in U} \frac{\left|\mu_{Y}(\cdot, u, \cdot)\right|}{1+\left|\sigma_{Y}(\cdot, u, \cdot)\right|} \quad \text { is locally bounded, }
$$

With stochastic Perron's method, we will show 
- in Section 5.3 that $V_{s}$ is the unique viscosity solution of

$$
\begin{gathered}
\min \left\{-\partial_{t} \varphi(t, x)+H \varphi(t, x), \varphi(t, x)-g(x)\right\}=0 \text { in } \mathbb{D}_{i}, \\
\varphi(T, x)=g(x) \text { for } x \in \mathbb{R}^{d} .
\end{gathered}
$$

- in Section 5.4 that $V_{b}$ is the unique viscosity solution of

$$
\begin{gathered}
\min \left\{-\partial_{t} \varphi(t, x)+F \varphi(t, x), \varphi(t, x)-g(x)\right\}=0 \text { in } \mathbb{D}_{i}, \\
\varphi(T, x)=g(x) \text { for } x \in \mathbb{R}^{d}
\end{gathered}
$$

\subsection{The super-hedging problem}

We now introduce classes of stochastic super-solutions and sub-solutions to (5.2.2), which envelope the value function and are closed under pairwise minimization and maximization, respectively.

Definition 5.3.1 (Stochastic super-solutions). A function $w:[0, T] \times \mathbb{R}^{d} \rightarrow \mathbb{R}$ is called a stochastic super-solution of (5.2.2) if

1. It is bounded, continuous and $w(t, x) \geq g(x)$ for all $(t, x) \in \mathbb{D}$.

2. For any $(t, x, y) \in \mathbb{D} \times \mathbb{R},\left\{\tau^{\alpha}\right\} \in \mathbb{T}_{t}$ and $\mathfrak{u} \in \mathfrak{U}(t)$, there exists $\tilde{\mathfrak{u}} \in \mathfrak{U}\left(t,\left\{\tau^{\alpha}\right\}\right)$ such that for all $\alpha \in \mathcal{A}^{t}$ and $\rho \in \mathcal{T}_{t}$ satisfying $\tau^{\alpha} \leq \rho \leq T$, we have

$$
Y(\rho) \geq w(\rho, X(\rho)) \mathbb{P}-\text { a.s. } \quad \text { on }\left\{Y\left(\tau^{\alpha}\right) \geq w\left(\tau^{\alpha}, X\left(\tau^{\alpha}\right)\right)\right\}
$$

where $X:=X_{t, x}^{\alpha}$ and $Y:=Y_{t, x, y}^{u \otimes_{\tau} \alpha \tilde{u}[\alpha], \alpha}$.

The set of stochastic super-solutions is denoted by $\mathbb{U}_{s}^{+}$.

Definition 5.3.2 (Stochastic sub-solutions). A function $w:[0, T] \times \mathbb{R}^{d} \rightarrow \mathbb{R}$ is called a stochastic sub-solution of (5.2.2) if

1. It is bounded, continuous and $w(T, x) \leq g(x)$ for all $x \in \mathbb{R}^{d}$. 
2. For any $(t, x, y) \in \mathbb{D} \times \mathbb{R},\left\{\tau^{\alpha}\right\} \in \mathbb{T}_{t}, \mathfrak{u} \in \mathfrak{U}(t)$ and $\alpha \in \mathcal{A}^{t}$, there exist $\widetilde{\alpha} \in \mathcal{A}^{t}$ and $\rho \in \mathcal{T}_{t}$ satisfying $\tau^{\alpha} \leq \rho \leq T\left(\widetilde{\alpha}\right.$ and $\rho$ may depend on $w, \mathfrak{u},\left\{\tau^{\alpha}\right\}$ and $\left.\alpha\right)$ such that

$$
\mathbb{P}(Y(\rho)<g(X(\rho)) \mid B)>0
$$

for any $B \subset\left\{Y\left(\tau^{\alpha}\right)<w\left(\tau^{\alpha}, X\left(\tau^{\alpha}\right)\right\}\right.$ satisfying $B \in \mathcal{F}_{\tau^{\alpha}}^{t}$ and $\mathbb{P}(B)>0$, where $X:=X_{t, x}^{\alpha \otimes_{\tau} \alpha \widetilde{\alpha}}$ and $Y:=Y_{t, x, y}^{\mathfrak{u}, \alpha \otimes_{\tau^{\alpha}} \widetilde{\alpha}}$.

The set of stochastic sub-solutions is denoted by $\mathbb{U}_{s}^{-}$.

Proposition 5.3.3. Under Assumptions 5.2.1, 5.2.12 and 5.2.13, $\mathbb{U}_{s}^{+}$is not empty.

Proof. The proposition follows from similar arguments to those in 4.2.14.

Assumption 5.3.4. $\mathbb{U}_{s}^{-}$are not empty.

Following similar arguments to those in Subsection 4.2.3, we can see that the following holds if $\mathbb{U}_{s}^{+}$and $\mathbb{U}_{s}^{-}$are not empty.

$$
V_{s}^{-}:=\sup _{w \in \mathbb{U}_{s}^{-}} w \leq V_{s} \leq \inf _{w \in \mathbb{U}_{s}^{+}} w=: V_{s}^{+}
$$

In the rest of the section, we will show in Theorem 5.3.7 that that $V_{s}^{+}$is a viscosity sub-solution and $V_{s}^{-}$is a super-solution of (5.2.2). We first state two lemmas without proof. For their proofs, we refer the readers to Lemma 4.3.1 and 4.3.2.

Lemma 5.3.5. $\mathbb{U}_{s}^{+}$(resp. $\mathbb{U}_{s}^{-}$) is closed under pairwise minimization (resp. maximization).

Lemma 5.3.6. There exists a non-increasing sequence $\mathbb{U}_{s}^{+} \ni w_{n} \searrow V_{s}^{+}$and a nondecreasing sequence $\mathbb{U}_{s}^{-} \ni v_{n} \nearrow V_{s}^{-}$.

Theorem 5.3.7. Let Assumptions 5.2.1 and 5.2.12 hold. 
1. If Assumption 5.2.13 holds, $V_{s}^{+}$is a bounded USC viscosity sub-solution of $(5.2 .2)$.

2. If Assumptions 5.2.14 and 5.3.4 hold, $V_{s}^{-}$is a bounded LSC viscosity supersolution of (5.2.2).

Proof. Step 1. ( $V_{s}^{+}$is a viscosity sub-solution)

1.1. The interior sub-solution property: Assume, on the contrary, that $\left(t_{0}, x_{0}\right) \in \mathbb{D}_{i}$ and $\varphi \in C^{1,2}(\mathbb{D})$ be such that

$$
0=V_{s}^{+}\left(t_{0}, x_{0}\right)-\varphi\left(t_{0}, x_{0}\right)=\max _{\mathbb{D}_{i}}\left(V_{s}^{+}(s, x)-\varphi(t, x)\right),
$$

$\varphi\left(t_{0}, x_{0}\right)>g\left(x_{0}\right)$ and $-\partial_{t} \varphi\left(t_{0}, x_{0}\right)+H \varphi\left(t_{0}, x_{0}\right)>0$. By similar arguments to those in Step 1.1 of Theorem 4.3.4's proof in Chapter IV, there exist $\varepsilon>0$ and $\delta>0$ such that

$$
\begin{gathered}
\varphi(t, x)>g(x)+\varepsilon \text { and }-\partial_{t} \varphi(t, x)+H\left(t, x, y, D \varphi(t, x), D^{2} \varphi(t, x)\right)>0 \\
\text { for all }(t, x) \in \bar{B}_{\varepsilon}\left(t_{0}, x_{0}\right) \text { and } y \in \mathbb{R} \text { s.t. }|y-\varphi(t, x)| \leq \delta
\end{gathered}
$$

Choose $\varepsilon$ small enough such that $\bar{B}_{\varepsilon}\left(t_{0}, x_{0}\right) \cap \mathbb{D}_{T}=\emptyset$. Since $\varphi>V_{s}^{+}$on the compact set $\mathbb{T}:=\bar{B}_{\varepsilon}\left(t_{0}, x_{0}\right)-B_{\varepsilon / 2}\left(t_{0}, x_{0}\right)$ and $V_{s}^{+}$is USC, $\varphi>V_{s}^{+}+2 \eta$ on $\mathbb{T}$ for some $\eta>0$. Let $\left\{w_{n}\right\}$ be a sequence such that $w_{n} \searrow V_{s}^{+}$. A Dini type argument shows that for large enough $n$,

$$
\varphi>w_{n}+\eta \text { on } \mathbb{T}, \varphi>w_{n}-\delta \text { on } \bar{B}_{\varepsilon / 2}\left(t_{0}, x_{0}\right)
$$

Fix such an $n$ and let $w=w_{n}$. For $\kappa \in(0, \eta \wedge \delta \wedge \varepsilon)$, define

$$
w^{\kappa}:=\left\{\begin{array}{l}
(\varphi-\kappa) \wedge w \text { on } \bar{B}_{\varepsilon}\left(t_{0}, x_{0}\right), \\
w \text { outside } \bar{B}_{\varepsilon}\left(t_{0}, x_{0}\right) .
\end{array}\right.
$$

Since $w^{\kappa}\left(t_{0}, x_{0}\right)=\varphi\left(t_{0}, x_{0}\right)-\kappa<V_{s}^{+}\left(t_{0}, x_{0}\right)$, we will obtain a contradiction if we can show $w^{\kappa} \in \mathbb{U}_{s}^{+}$. Obviously, $w^{\kappa}$ is continuous and bounded. Since $w \in \mathbb{U}_{s}^{+}$and 
$\varphi(t, x)-\kappa>g(x)+\varepsilon-\kappa>g(x)$ for all $(t, x) \in \bar{B}_{\varepsilon}\left(t_{0}, x_{0}\right), w^{\kappa}(t, x) \geq g(x)$ for all $(t, x) \in \mathbb{D}$. The rest of the proof (which shows that $w^{\kappa}$ satisfies the second property of Definition 5.3.1) is the same as that in Step 1.1 of Theorem 4.3.4 in Chapter IV.

1.2. The boundary condition: The proof follows the same arguments in Step 1.2 of Theorem 4.3.4's proof in Chapter IV.

\section{Step 2. ( $V_{s}^{-}$is a viscosity super-solution)}

2.1. $\boldsymbol{V}_{\boldsymbol{s}}^{-}(\boldsymbol{t}, \boldsymbol{x}) \geq \boldsymbol{g}(\boldsymbol{x})$ : In this step, we show that $V_{s}^{-}(t, x) \geq g(x)$ for all $(t, x) \in \mathbb{D}$. Assume, on the contrary, that for some $\left(t_{0}, x_{0}\right) \in \mathbb{D}$ there exists $\eta>0$ such that

$$
0<2 \eta:=g\left(x_{0}\right)-V_{s}^{-}\left(t_{0}, x_{0}\right)
$$

Take an arbitrary $w \in \mathbb{U}_{s}^{-}$. From the fact that $w\left(t_{0}, x_{0}\right) \leq V_{s}^{-}\left(t_{0}, x_{0}\right)$, the continuity of $w$ and lower semi-continuity of $g$, there exists $\varepsilon>0$ such that for all $(t, x) \in$ $\bar{B}_{\varepsilon}\left(t_{0}, x_{0}\right)$,

$$
g(x)-w(t, x)>\eta, g(x)-g\left(x_{0}\right)>-\frac{\eta}{2} \text { and }\left|w(t, x)-w\left(t_{0}, x_{0}\right)\right|<\frac{\eta}{2} .
$$

Define

$$
w^{\prime}(t, x):=\left\{\begin{array}{l}
w(t, x) \text { for }(t, x) \notin \bar{B}_{\varepsilon}\left(t_{0}, x_{0}\right) \\
w(t, x)+\left(1-\frac{\operatorname{dist}\left((t, x),\left(t_{0}, x_{0}\right)\right)}{\varepsilon}\right)\left(g\left(x_{0}\right)-\eta-w\left(t_{0}, x_{0}\right)\right), \text { otherwise. }
\end{array}\right.
$$

Obviously, $w^{\prime}$ is bounded, continuous and

$$
\left\{(t, x): w(t, x)<w^{\prime}(t, x)\right\} \subset \bar{B}_{\varepsilon}\left(t_{0}, x_{0}\right)
$$

Moreover, by (5.3.4),

$$
w^{\prime}(t, x) \leq w(t, x)+g\left(x_{0}\right)-\eta-w\left(t_{0}, x_{0}\right)<g(x) \text { for }(t, x) \in \bar{B}_{\varepsilon}\left(t_{0}, x_{0}\right)
$$

The equation above, along with the fact that $w \in \mathbb{U}_{s}^{-}$, implies that $w^{\prime}(T, x) \leq g(x)$.

Since $w^{\prime}\left(t_{0}, x_{0}\right)=g\left(x_{0}\right)-\eta>V_{s}^{-}\left(t_{0}, x_{0}\right)$ by (5.3.3), we will obtain a contradiction if $w^{\prime} \in \mathbb{U}_{s}^{-}$. 
We now show that $w^{\prime} \in \mathbb{U}_{s}^{-}$. Fix $(t, x, y) \in \mathbb{D}_{i} \times \mathbb{R},\left\{\tau^{\alpha}\right\} \in \mathbb{T}_{t}, \mathfrak{u} \in \mathfrak{U}(t)$ and $\alpha \in \mathcal{A}^{t}$. Let $\widetilde{\alpha}^{w} \in \mathcal{A}^{t}$ and $\rho^{w} \in \mathcal{T}_{t}$ be the "optimal" control and stopping time of Nature in Definition 5.3.2 for $w^{2}$ Define

$$
\widetilde{\alpha}:=\left(\widetilde{\alpha}^{w} \mathbb{1}_{A}+\alpha_{0} \mathbb{1}_{A^{c}}\right) \mathbb{1}_{\left[\tau^{\alpha}, T\right]} \quad \text { and } \quad \rho:=\rho^{w} \mathbb{1}_{A}+\tau^{\alpha} \mathbb{1}_{A^{c}}
$$

where $\alpha_{0}$ is arbitrary in $\mathcal{A}^{t}$ and $A=\left\{w^{\prime}\left(\tau^{\alpha}, X_{t, x}^{\alpha}\left(\tau^{\alpha}\right)\right)=w\left(\tau^{\alpha}, X_{t, x}^{\alpha}\left(\tau^{\alpha}\right)\right)\right\}$. We can easily check that $\widetilde{\alpha} \in \mathcal{A}^{t}$ and $\rho \in \mathcal{T}_{t}$ with $\rho \geq \tau^{\alpha}$. Let $X:=X_{t, x}^{\alpha \otimes_{\tau^{\alpha}} \widetilde{\alpha}}$ and $Y:=Y_{t, x, y}^{\mathfrak{u}, \alpha \otimes_{\tau} \alpha \widetilde{\alpha}}$. It suffices to show that

$$
\mathbb{P}(Y(\rho)<g(X(\rho)) \mid B)>0
$$

for any $B \subset\left\{Y\left(\tau^{\alpha}\right)<w^{\prime}\left(\tau^{\alpha}, X\left(\tau^{\alpha}\right)\right\}\right.$ satisfying $B \in \mathcal{F}_{\tau^{\alpha}}^{t}$ and $\mathbb{P}(B)>0$. Note that

$$
\begin{gathered}
X\left(\tau^{\alpha}\right)=X_{t, x}^{\alpha}\left(\tau^{\alpha}\right), \quad Y\left(\tau^{\alpha}\right)=Y_{t, x, y}^{\mathfrak{u}, \alpha}\left(\tau^{\alpha}\right), \\
X(s)=\mathbb{1}_{A} X_{t, x}^{\alpha \otimes_{\tau^{\alpha}} \widetilde{\alpha}^{w}}(s)+\mathbb{1}_{A^{c}} X_{t, x}^{\alpha \otimes_{\tau^{\alpha} \alpha_{0}}}(s) \quad \text { for } \tau^{\alpha} \leq s, \\
Y(s)=\mathbb{1}_{A} Y_{t, x, y}^{\mathfrak{u}, \alpha \otimes_{\tau^{\alpha}} \widetilde{\alpha}^{w}}(s)+\mathbb{1}_{A^{c}} Y_{t, x, y}^{\mathfrak{u}, \alpha \otimes_{\tau} \alpha \alpha_{0}}(s) \quad \text { for } \tau^{\alpha} \leq s .
\end{gathered}
$$

We consider the following two cases which will yield the desired result.

(i) If $\mathbb{P}(B \cap A)>0$ : Note that

$$
B \cap A \subset\left\{Y_{t, x, y}^{\mathfrak{u}, \alpha \otimes_{\tau^{\alpha}} \widetilde{\alpha}^{w}}\left(\tau^{\alpha}\right)<w\left(\tau^{\alpha}, X_{t, x}^{\alpha \otimes_{\tau^{\alpha}} \widetilde{\alpha}^{w}}\left(\tau^{\alpha}\right)\right)\right\}
$$

From the fact $w \in \mathbb{U}_{s}^{-}$, the equation above, (5.3.8) and the definitions of $\rho$ and $\widetilde{\alpha}^{w}$ on $A$,

$$
\mathbb{P}(Y(\rho)<g(X(\rho)) \mid B \cap A)=\mathbb{P}\left(Y_{t, x, y}^{\mathfrak{u}, \alpha \otimes_{\tau} \alpha \widetilde{\alpha}^{w}}\left(\rho^{w}\right)<g\left(X_{t, x}^{\alpha \otimes_{\tau^{\alpha}} \widetilde{\alpha}^{w}}\left(\rho^{w}\right)\right) \mid B \cap A\right)>0
$$

This implies that $\mathbb{P}(\{Y(\rho)<g(X(\rho))\} \cap B \cap A)>0$.

(ii) If $\mathbb{P}\left(B \cap A^{c}\right)>0$ : By (5.3.5) and (5.3.6),

$$
Y_{t, x, y}^{\mathfrak{u}, \alpha}\left(\tau^{\alpha}\right)<w^{\prime}\left(\tau^{\alpha}, X_{t, x}^{\alpha}\left(\tau^{\alpha}\right)\right)<g\left(X_{t, x}^{\alpha}\left(\tau^{\alpha}\right)\right) \text { on } B \cap A^{c}
$$

\footnotetext{
${ }^{2}$ Although the "optimal" control and stopping time also depend on $\left\{\tau^{\alpha}\right\}, \mathfrak{u}$ and $\alpha$, we only emphasize the dependence on $w$.
} 
By (5.3.7), (5.3.8) and the definition of $\rho$ on $A^{c}$,

$$
\mathbb{P}\left(Y(\rho)<g(X(\rho)) \mid B \cap A^{c}\right)=\mathbb{P}\left(Y_{t, x, y}^{\mathfrak{u}, \alpha}\left(\tau^{\alpha}\right)<g\left(X_{t, x}^{\alpha}\left(\tau^{\alpha}\right)\right) \mid B \cap A^{c}\right)>0 .
$$

This further implies that $\mathbb{P}\left(\{Y(\rho)<g(X(\rho))\} \cap B \cap A^{c}\right)>0$.

$2.2-\partial_{t} V_{s}^{-}(t, x)+H V_{s}^{-}(t, x) \geq 0$ on $\mathbb{D}_{i}$ in the viscosity sense. Assume, on the contrary, that $\left(t_{0}, x_{0}\right) \in \mathbb{D}_{i}$ and $\varphi \in C^{1,2}(\mathbb{D})$ are such that

$$
\begin{gathered}
\max _{(t, x) \in \mathbb{D}}\left(\varphi(t, x)-V_{s}^{-}(t, x)\right)=\varphi\left(t_{0}, x_{0}\right)-V_{s}^{-}\left(t_{0}, x_{0}\right)=0 \text { and } \\
-\partial_{t} \varphi\left(t_{0}, x_{0}\right)+H \varphi\left(t_{0}, x_{0}\right)<0 .
\end{gathered}
$$

From the equation above, there exists an $a_{0} \in A$ such that

$$
-\partial_{t} \varphi\left(t_{0}, x_{0}\right)+L^{u_{0}, a_{0}} \varphi\left(t_{0}, x_{0}\right)<0,
$$

where $u_{0}:=\hat{u}\left(t_{0}, x_{0}, \varphi\left(t_{0}, x_{0}\right), \sigma_{X}\left(t_{0}, x_{0}, a_{0}\right) D \varphi\left(t_{0}, x_{0}\right), a_{0}\right)$. From the continuity of $\mu_{X}, \mu_{Y}$ and $\sigma_{X}$ in Assumption 5.2.1 and the continuity of $\hat{u}$ in Assumption 5.2.12, there exist $\varepsilon>0$ and $\delta>0$ such that

$$
\begin{gathered}
-\partial_{t} \varphi(t, x)+L^{u, a_{0}}\left(t, x, y, D \varphi(t, x), D^{2} \varphi(t, x)\right)<0, \text { for }(t, x) \in \bar{B}_{\varepsilon}\left(t_{0}, x_{0}\right) \text { and }(y, u) \\
\in R \times U \text { s.t. }|y-\varphi(t, x)| \leq \delta \text { and }\left|\sigma_{Y}\left(t, x, y, u, a_{0}\right)-\sigma_{X}\left(t, x, a_{0}\right) D \varphi(t, x)\right| \leq \delta
\end{gathered}
$$

Choose $\varepsilon$ small enough to make sure that $\bar{B}_{\varepsilon}\left(t_{0}, x_{0}\right) \cap \mathbb{D}_{T}=\emptyset$. Since $\varphi<V_{s}^{-}$on the compact set $\mathbb{T}:=\bar{B}_{\varepsilon}\left(t_{0}, x_{0}\right)-B_{\varepsilon / 2}\left(t_{0}, x_{0}\right)$ and $V_{s}^{-}$is LSC, $\varphi<V_{s}^{-}-2 \eta$ on $\mathbb{T}$ for some $\eta>0$. Let $\left\{w_{n}\right\}$ be a sequence in $\mathbb{U}_{s}^{-}$such that $w_{n} \nearrow V_{s}^{-}$. By a Dini type argument, there exists $n_{0} \in \mathbb{N}$ such that for all $n \geq n_{0}$,

$$
\varphi<w_{n}-\eta \text { on } \mathbb{T}, \varphi<w_{n}+\delta \text { on } \bar{B}_{\varepsilon / 2}\left(t_{0}, x_{0}\right) .
$$

Fix such an $n$ and let $w=w_{n}$. For $\kappa \in(0, \eta \wedge \delta \wedge \varepsilon)$, define

$$
w^{\kappa}:=\left\{\begin{array}{l}
(\varphi+\kappa) \vee w \text { on } \bar{B}_{\varepsilon}\left(t_{0}, x_{0}\right), \\
w \text { outside } \bar{B}_{\varepsilon}\left(t_{0}, x_{0}\right) .
\end{array}\right.
$$


Since $w^{\kappa}\left(t_{0}, x_{0}\right)=\varphi\left(t_{0} x_{0}\right)+\kappa>V_{s}^{-}\left(t_{0}, x_{0}\right)$, we would obtain a contradiction if we could show $w^{\kappa} \in \mathbb{U}_{s}^{-}$. Obviously, $w^{\kappa}$ is continuous, bounded and $w^{\kappa}(T, x) \geq g(x)$ for $x \in \mathbb{R}^{d}$.

Fix $(t, x, y),\left\{\tau^{\alpha}\right\} \in \mathbb{T}_{t}, \mathfrak{u} \in \mathfrak{U}(t)$ and $\alpha \in \mathcal{A}^{t}$. To show $w^{\kappa} \in \mathbb{U}_{s}^{-}$, we need to construct an "optimal" control $\widetilde{\alpha} \in \mathcal{A}^{t}$ and an "optimal" stopping time $\rho \in \mathcal{T}_{t}$ satisfying $\rho \geq \tau^{\alpha}$ in the sense of Definition 5.3.2 for $w^{\kappa}$. Let $A=\left\{w\left(\tau^{\alpha}, X_{t, x}^{\alpha}\left(\tau^{\alpha}\right)\right)=\right.$ $\left.w^{\kappa}\left(\tau^{\alpha}, X_{t, x}^{\alpha}\left(\tau^{\alpha}\right)\right)\right\}$. We consider the construction of $\tilde{\alpha}$ and $\rho$ on $A$ and $A^{c}$ separately:

(i) On $A$ : Let $\widetilde{\alpha}^{w, \tau^{\alpha}}$ and $\rho^{w, \tau^{\alpha}}$ be the "optimal" control and stopping time of Nature for $w$ in Definition 5.3.2 given $\mathfrak{u}, \alpha$ and $\left\{\tau^{\alpha}\right\}$. Set $\widetilde{\alpha}=\widetilde{\alpha}^{w, \tau^{\alpha}}$ and $\rho=\rho^{w, \tau^{\alpha}}$.

(ii) On $A^{c}$ : Let $\theta^{\alpha}=\theta_{1}^{\alpha} \wedge \theta_{2}^{\alpha}$, where

$$
\begin{gathered}
\theta_{1}^{\alpha}:=\inf \left\{s \in\left[\tau^{\alpha}, T\right]:\left(s, X_{t, x}^{\alpha \otimes_{\tau^{\alpha}} a_{0}}(s)\right) \notin B_{\varepsilon / 2}\left(t_{0}, x_{0}\right)\right\} \wedge T \text { and } \\
\theta_{2}^{\alpha}:=\inf \left\{s \in\left[\tau^{\alpha}, T\right]:\left|Y_{t, x, y}^{\mathfrak{u}, \alpha \otimes_{\tau^{\alpha} a_{0}}}(s)-\varphi\left(s, X_{t, x}^{\alpha \otimes_{\tau} \alpha a_{0}}(s)\right)\right| \geq \delta\right\} \wedge T .
\end{gathered}
$$

By Example 1 in [4], $\left\{\theta^{\alpha}\right\} \in \mathbb{T}_{t}$. Let $\widetilde{\alpha}$ be $a_{0}$ on $\left[\tau^{\alpha}, \theta^{\alpha}\right)$. Starting from $\theta^{\alpha}$, choose $\widetilde{\alpha}=\widetilde{\alpha}^{w, \theta^{\alpha}}$ and $\rho=\rho^{w, \theta^{\alpha}}$, where $\widetilde{\alpha}^{w, \theta^{\alpha}}$ is the "optimal" control and $\rho^{w, \theta^{\alpha}}$ is the "optimal" stopping time satisfying $\rho^{w, \theta^{\alpha}} \geq \theta^{\alpha}$ which correspond to $\mathfrak{u}, \alpha \otimes_{\tau^{\alpha}} a_{0}$ and $\left\{\theta^{\alpha}\right\}$ in Definition 5.3.2.

In short,

$$
\widetilde{\alpha}=\left(\mathbb{1}_{A} \widetilde{\alpha}^{w, \tau}+\mathbb{1}_{A^{c}}\left(a_{0} \mathbb{1}_{\left[t, \theta^{\alpha}\right)}+\widetilde{\alpha}^{w, \theta} \mathbb{1}_{\left[\theta^{\alpha}, T\right]}\right)\right) \mathbb{1}_{\left[\tau^{\alpha}, T\right]}, \rho=\mathbb{1}_{A} \rho^{w, \tau}+\mathbb{1}_{A^{c}} \rho^{w, \theta}
$$

To show the construction works, the proof follows from similar arguments to those in Step 2.1 of Theorem 4.3.4's proof.

To characterize $V_{s}$ as the unique viscosity solution of (5.2.2), we need a comparison principle. 
Proposition 5.3.8. Under Assumptions 5.2.1, 5.2.12 and 5.2.13, the comparison principle for (5.2.2) holds. More precisely, if $U$ (resp. W) is a bounded LSC viscosity sub-solution (resp. a bounded USC viscosity super-solution) to (5.2.2) and $U \leq W$ on $\mathbb{D}_{T}$, then $U \leq W$ on $\mathbb{D}$.

Proof. This proposition follows from the arguments in Proposition 4.3.5.

Corollary 5.3.9. Under Assumptions 5.2.1, 5.2.12, 5.2.13, 5.2.14 and 5.3.4, $V_{s}$ is the unique bounded continuous viscosity solution of (5.2.2).

Proof. This corollary follows from the same arguments in Corollary 4.3.6.

\subsection{The sub-hedging problem}

Definition 5.4.1 (Stochastic super-solutions). A function $w:[0, T] \times \mathbb{R}^{d} \rightarrow \mathbb{R}$ is called a stochastic super-solution of (5.2.3) if

1. It is bounded, continuous and $w(t, x) \geq g(x)$ for all $(t, x) \in \mathbb{D}$.

2. For any $(t, x, y) \in \mathbb{D} \times \mathbb{R},\left\{\tau^{\alpha}\right\} \in \mathbb{T}_{t}, \mathfrak{u} \in \mathfrak{U}(t), \boldsymbol{\rho} \in \mathbf{T}\left(t,\left\{\tau^{\alpha}\right\}\right)$ and $\alpha \in \mathcal{A}^{t}$, there exists $\widetilde{\alpha} \in \mathcal{A}^{t}$ such that

$$
\mathbb{P}(Y(\boldsymbol{\rho}[\alpha])>w(\boldsymbol{\rho}[\alpha], X(\boldsymbol{\rho}[\alpha])) \mid B)>0
$$

for any $B \subset\left\{Y\left(\tau^{\alpha}\right)>w\left(\tau^{\alpha}, X\left(\tau^{\alpha}\right)\right\}\right.$ satisfying $B \in \mathcal{F}_{\tau^{\alpha}}^{t}$ and $\mathbb{P}(B)>0$, where $X:=X_{t, x}^{\alpha \otimes_{\tau} \alpha \widetilde{\alpha}}$ and $Y:=Y_{t, x, y}^{\mathfrak{u}, \alpha \otimes_{\tau} \alpha \widetilde{\alpha}}$.

The set of stochastic sub-solutions is denoted by $\mathbb{U}_{b}^{+}$.

Definition 5.4.2 (Stochastic sub-solutions). A function $w:[0, T] \times \mathbb{R}^{d} \rightarrow \mathbb{R}$ is called a stochastic sub-solution of (5.2.3) if

1. It is bounded, continuous and $w(T, x) \leq g(x)$ for all $x \in \mathbb{R}^{d}$. 
2. For $(t, x, y) \in \mathbb{D} \times \mathbb{R},\left\{\tau^{\alpha}\right\} \in \mathbb{T}_{t}$ and $\mathfrak{u} \in \mathfrak{U}(t)$, there exist $\tilde{\mathfrak{u}} \in \mathfrak{U}\left(t,\left\{\tau^{\alpha}\right\}\right)$ and $\boldsymbol{\rho} \in \mathbf{T}\left(t,\left\{\tau^{\alpha}\right\}\right)$ such that for any $\alpha \in \mathcal{A}^{t}$,

$$
Y(\boldsymbol{\rho}[\alpha]) \leq g(X(\boldsymbol{\rho}[\alpha])) \mathbb{P}-\text { a.s. } \quad \text { on }\left\{Y\left(\tau^{\alpha}\right) \leq w\left(\tau^{\alpha}, X\left(\tau^{\alpha}\right)\right)\right\}
$$

where $X:=X_{t, x}^{\alpha}$ and $Y:=Y_{t, x, y}^{\mathfrak{u} \otimes_{\tau}^{\alpha \tilde{u}}[\alpha], \alpha}$.

The set of stochastic super-solutions is denoted by $\mathbb{U}_{b}^{-}$.

Proposition 5.4.3. Under Assumptions 5.2.1, 5.2.12, 5.2.13, $\mathbb{U}_{b}^{-}$is not empty.

Assumption 5.4.4. $\mathbb{U}_{b}^{+}$is not empty.

Following similar arguments to those in Subsection 4.2.3, we can check that

$$
V_{b}^{-}:=\sup _{w \in \mathbb{U}_{b}^{-}} w \leq V_{b} \leq \inf _{w \in \mathbb{U}_{b}^{+}} w=: V_{b}^{+}
$$

when $\mathbb{U}_{b}^{+}$and $\mathbb{U}_{b}^{-}$are not empty. Next we will show in Theorem 5.4.7 that $V_{b}^{+}$is a viscosity sub-solution and $V_{b}^{-}$is a super-solution of (5.2.3). We still have the following two preparatory lemmas.

Lemma 5.4.5. $\mathbb{U}_{b}^{+}$(resp. $\left.\mathbb{U}_{b}^{-}\right)$is closed under pairwise minimization (resp. maximization).

Lemma 5.4.6. There exists a non-increasing sequence $\mathbb{U}_{b}^{+} \ni w_{n} \searrow V_{b}^{+}$and a nondecreasing sequence $\mathbb{U}_{b}^{-} \ni v_{n} \nearrow V_{b}^{-}$.

Theorem 5.4.7. Let Assumptions 5.2.1 and 5.2.12 hold.

1. If Assumption 5.2.13 holds, $V_{b}^{+}$is a bounded USC viscosity sub-solution of $(5.2 .3)$

2. If Assumptions 5.2.14 and 5.4.4 hold in addition to the main assumptions, $V_{b}^{-}$ is a bounded LSC viscosity super-solution of (5.2.3). 


\section{Proof. Step 1. ( $V_{b}^{-}$is a viscosity super-solution)}

1.1. $\boldsymbol{V}_{\boldsymbol{b}}^{-}(\boldsymbol{t}, \boldsymbol{x}) \geq \boldsymbol{g}(\boldsymbol{x})$ : We show that $V_{b}^{-}(t, x) \geq g(x)$ for all $(t, x) \in \mathbb{D}$ in this step. Assume, on the contrary, that for some $\left(t_{0}, x_{0}\right) \in \mathbb{D}$, there exists $\eta>0$ such that

$$
-2 \eta:=V_{b}^{-}\left(t_{0}, x_{0}\right)-g\left(x_{0}\right)<0 .
$$

Take an arbitrary $w \in \mathbb{U}_{b}^{-}$. From the fact that $w\left(t_{0}, x_{0}\right) \leq V_{b}^{-}\left(t_{0}, x_{0}\right)$, the continuity of $w$ and upper semi-continuity of $g$, there exists $\varepsilon>0$ such that for all $(t, x) \in$ $\bar{B}_{\varepsilon}\left(t_{0}, x_{0}\right)$

$$
w(t, x)-g(x)<-\eta, g(x)-g\left(x_{0}\right)>-\frac{\eta}{2} \text { and }\left|w(t, x)-w\left(t_{0}, x_{0}\right)\right|<\frac{\eta}{2} .
$$

Define

$$
w^{\prime}(t, x):=\left\{\begin{array}{l}
w(t, x) \text { for }(t, x) \notin \bar{B}_{\varepsilon}\left(t_{0}, x_{0}\right), \\
w(t, x)+\left(1-\frac{\operatorname{dist}\left((t, x),\left(t_{0}, x_{0}\right)\right)}{\varepsilon}\right)\left(g\left(x_{0}\right)-w\left(t_{0}, x_{0}\right)-\eta\right), \text { otherwise. }
\end{array}\right.
$$

Obviously, $w^{\prime}$ is bounded and continuous. Moreover,

$$
w^{\prime}(t, x) \leq w(t, x)+\left(g\left(x_{0}\right)-w\left(t_{0}, x_{0}\right)-\eta\right)<g(x) \text { for }(t, x) \in \bar{B}_{\varepsilon}\left(t_{0}, x_{0}\right) .
$$

This, together with the fact that $w(T, x) \leq g(x)$ for all $x \in \mathbb{R}^{d}$, implies that $w^{\prime}(T, x) \leq g(x)$. Since $w^{\prime}\left(t_{0}, x_{0}\right)=g\left(x_{0}\right)-\eta>V_{b}^{-}\left(t_{0}, x_{0}\right)$, we will obtain a contradiction if $w^{\prime} \in \mathbb{U}_{b}^{-}$.

Fix $(t, x, y) \in \mathbb{D}_{i} \times \mathbb{R},\left\{\tau^{\alpha}\right\} \in \mathbb{T}_{t}$ and $\mathfrak{u} \in \mathfrak{U}(t)$. Let $\widetilde{\mathfrak{u}}^{w} \in \mathfrak{U}\left(t,\left\{\tau^{\alpha}\right\}\right)$ and $\boldsymbol{\rho}^{w} \in$ $\mathbf{T}\left(t,\left\{\tau^{\alpha}\right\}\right)$ be the strategies in Definition 5.4.1 for $w$. Define

$$
\widetilde{\mathfrak{u}}[\alpha]=\left(\widetilde{\mathfrak{u}}^{w}[\alpha] \mathbb{1}_{A}+\mathfrak{u}^{*}[\alpha] \mathbb{1}_{A^{c}}\right) \mathbb{1}_{\left[\tau^{\alpha}, T\right]} \quad \text { and } \quad \boldsymbol{\rho}[\alpha]=\boldsymbol{\rho}^{w}[\alpha] \mathbb{1}_{A}+\tau^{\alpha} \mathbb{1}_{A^{c}}
$$


where $\mathfrak{u}^{*}$ is arbitrary in $\mathfrak{U}\left(t,\left\{\tau^{\alpha}\right\}\right)$ and $A=\left\{w^{\prime}\left(\tau^{\alpha}, X_{t, x}^{\alpha}\left(\tau^{\alpha}\right)\right)=w\left(\tau^{\alpha}, X_{t, x}^{\alpha}\left(\tau^{\alpha}\right)\right)\right\}$. It is easy to check that $\widetilde{\mathfrak{u}} \in \mathfrak{U}\left(t,\left\{\tau^{\alpha}\right\}\right)$ and $\boldsymbol{\rho} \in \mathbf{T}\left(t,\left\{\tau^{\alpha}\right\}\right)$. Let $X:=X_{t, x}^{\alpha}$ and $Y:=Y_{t, x, y}^{\mathfrak{u} \otimes_{\tau} \alpha \mathfrak{u}[\alpha], \alpha}$. It suffices to show that

$$
Y(\boldsymbol{\rho}[\alpha]) \leq g(X(\boldsymbol{\rho}[\alpha])) \mathbb{P}-\text { a.s. } \quad \text { on }\left\{Y\left(\tau^{\alpha}\right) \leq w^{\prime}\left(\tau^{\alpha}, X\left(\tau^{\alpha}\right)\right)\right\}
$$

Note that

$$
X\left(\tau^{\alpha}\right)=X_{t, x}^{\alpha}\left(\tau^{\alpha}\right), \quad Y\left(\tau^{\alpha}\right)=Y_{t, x, y}^{\mathfrak{u}, \alpha}\left(\tau^{\alpha}\right)
$$

We consider the following two cases.

(i) On the set $A \cap\left\{Y\left(\tau^{\alpha}\right) \leq w^{\prime}\left(\tau^{\alpha}, X\left(\tau^{\alpha}\right)\right)\right\}$ : Note that

$$
A \cap\left\{Y\left(\tau^{\alpha}\right) \leq w^{\prime}\left(\tau^{\alpha}, X\left(\tau^{\alpha}\right)\right)\right\} \subset\left\{Y\left(\tau^{\alpha}\right) \leq w\left(\tau^{\alpha}, X\left(\tau^{\alpha}\right)\right)\right\}
$$

The equation above, along with the definitions of $\widetilde{\mathfrak{u}}$ and $\boldsymbol{\rho}$ on $A$ and the fact $w \in \mathbb{U}_{b}^{-}$, implies that

$$
Y(\boldsymbol{\rho}[\alpha]) \leq g(X(\boldsymbol{\rho}[\alpha])) \text { on } A \cap\left\{Y\left(\tau^{\alpha}\right) \leq w^{\prime}\left(\tau^{\alpha}, X\left(\tau^{\alpha}\right)\right)\right\}
$$

(ii) On the set $A^{c} \cap\left\{Y\left(\tau^{\alpha}\right) \leq w^{\prime}\left(\tau^{\alpha}, X\left(\tau^{\alpha}\right)\right)\right\}$ : by (5.4.2),

$$
\left(\tau^{\alpha}, X_{t, x}^{\alpha}\left(\tau^{\alpha}\right)\right) \in \bar{B}_{\varepsilon}\left(t_{0}, x_{0}\right) \text { on } A^{c} \cap\left\{Y\left(\tau^{\alpha}\right) \leq w^{\prime}\left(\tau^{\alpha}, X\left(\tau^{\alpha}\right)\right)\right\}
$$

This implies from the definition of $\boldsymbol{\rho}$ on $A^{c},(5.4 .3)$ and (5.4.4) that

$$
Y_{t, x, y}^{\mathfrak{u}, \alpha}(\boldsymbol{\rho}[\alpha]) \leq w^{\prime}\left(\boldsymbol{\rho}[\alpha], X_{t, x}^{\alpha}(\boldsymbol{\rho}[\alpha])\right) \leq g\left(X_{t, x}^{\alpha}(\boldsymbol{\rho}[\alpha])\right)
$$

on $A^{c} \cap\left\{Y\left(\tau^{\alpha}\right) \leq w^{\prime}\left(\tau^{\alpha}, X\left(\tau^{\alpha}\right)\right)\right\}$. Therefore, (i) and (ii) yield the desired result.

1.2. $-\partial V_{b}^{-}(t, x)+F V_{b}^{-}(t, x) \geq 0$ on $\mathbb{D}_{i}$ in the viscosity sense. Let $\left(t_{0}, x_{0}\right) \in \mathbb{D}_{i}$ and $\varphi \in C^{1,2}(\mathbb{D})$ be such

$$
0=V_{b}^{-}\left(t_{0}, x_{0}\right)-\varphi\left(t_{0}, x_{0}\right)=\min _{(t, x) \in \mathbb{D}_{i}}\left(V_{b}^{-}(t, x)-\varphi(t, x)\right)
$$


Assume by contradiction that

$$
-\partial_{t}\left(t_{0}, x_{0}\right)+F \varphi\left(t_{0}, x_{0}\right)<0 \text {. }
$$

By applying similar arguments to those in Step 1.1 of Theorem 4.3.4's proof, there exist $\varepsilon>0$ and $\delta>0$ such that for all $(t, x, y) \in \mathbb{D} \times \mathbb{R}$ satisfying $(t, x) \in \bar{B}_{\varepsilon}\left(t_{0}, x_{0}\right)$ and $|y-\varphi(t, x)| \leq \delta$

$$
-\partial_{t} \varphi(t, x)+F\left(t, x, y, D \varphi(t, x), D^{2} \varphi(t, x)\right)<0
$$

Since $\varphi<V_{b}^{-}$on the compact set $\mathbb{T}:=\bar{B}_{\varepsilon}\left(t_{0}, x_{0}\right)-B_{\varepsilon / 2}\left(t_{0}, x_{0}\right)$ and $V_{b}^{-}$is LSC, $\varphi<V_{b}^{-}-2 \eta$ on $\mathbb{T}$ for some $\eta>0$. Let $\left\{w_{n}\right\}$ be a sequence in $\mathbb{U}_{b}^{-}$such that $w_{n} \nearrow V_{b}^{-}$. A Dini type argument shows that $\varphi<w_{n}-\eta$ on $\mathbb{T}$ and $\varphi<w_{n}+\delta$ on $\bar{B}_{\varepsilon / 2}\left(t_{0}, x_{0}\right)$ for large enough $n \in \mathbb{N}$. For simplicity, fix such an $n$ and let $w=w_{n}$. For $\kappa \in(0, \eta \wedge \delta \wedge \varepsilon)$, define

$$
w^{\kappa}:=\left\{\begin{array}{l}
(\varphi+\kappa) \vee w \text { on } \bar{B}_{\varepsilon}\left(t_{0}, x_{0}\right), \\
w \text { outside } \bar{B}_{\varepsilon}\left(t_{0}, x_{0}\right) .
\end{array}\right.
$$

Since $w^{\kappa}\left(t_{0}, x_{0}\right)=\varphi\left(t_{0}, x_{0}\right)+\kappa>V_{b}^{-}\left(t_{0}, x_{0}\right)$, we will obtain a contradiction if we can show $w^{\kappa} \in \mathbb{U}_{b}^{-}$. Obviously, $w^{\kappa}$ is continuous, bounded and $w^{\kappa}(T, x) \leq$ $g(x)$ for all $x \in \mathbb{R}^{d}$. Fix $(t, x, y) \in \mathbb{D}_{i} \times \mathbb{R},\left\{\tau^{\alpha}\right\} \in \mathbb{T}_{t}$ and $\mathfrak{u} \in \mathfrak{U}(t)$. Let $\tilde{\mathfrak{u}}^{w,\left\{\tau^{\alpha}\right\}} \in \mathfrak{U}\left(t,\left\{\tau^{\alpha}\right\}\right)$ be the "optimal" strategy in Definition 5.4.1 for $w$ and $A=$ $\left\{w^{\kappa}\left(\tau^{\alpha}, X_{t, x}^{\alpha}\left(\tau^{\alpha}\right)\right)=w\left(\tau^{\alpha}, X_{t, x}^{\alpha}\left(\tau^{\alpha}\right)\right)\right\}$. We construct $\tilde{\mathfrak{u}}$ on $A$ and $A^{c}$ separately:

(i) On $A$ : set $\tilde{\mathfrak{u}}$ to be $\tilde{\mathfrak{u}}^{w,\left\{\tau^{\alpha}\right\}}$.

(ii) On $A^{c}$ : For $\alpha \in \mathcal{A}^{t}$, let $\bar{Y}^{\alpha}$ be the unique strong solution (which is thanks in particular to Assumption 5.2.13) of the equation

$$
\begin{aligned}
\bar{Y}^{\alpha}(l) & =Y_{t, x, y}^{\mathfrak{u}, \alpha}\left(\tau^{\alpha}\right)+\int_{\tau^{\alpha}}^{\tau^{\alpha} \vee l} \sigma_{X}\left(s, X_{t, x}^{\alpha}(s), \alpha_{s}\right) D \varphi\left(s, X_{t, x}^{\alpha}(s)\right) d W_{s} \\
& +\int_{\tau^{\alpha}}^{\tau^{\alpha} \vee l} \mu_{Y}^{\hat{u}}\left(s, X_{t, x}^{\alpha}(s), \bar{Y}^{\alpha}(s), \sigma_{X}\left(s, X_{t, x}^{\alpha}(s), \alpha_{s}\right) D \varphi\left(s, X_{t, x}^{\alpha}(s)\right), \alpha_{s}\right) d s .
\end{aligned}
$$


Note that $\bar{Y}^{\alpha}(s)=Y_{t, x, y}^{\mathfrak{u}, \alpha}(s)$ for $s<\tau^{\alpha}$. Define

$$
\tilde{\mathfrak{u}}_{0}:=\tilde{\mathfrak{u}}_{0}[\alpha](s)=\hat{u}\left(s, X_{t, x}^{\alpha}(s), \bar{Y}^{\alpha}(s), \sigma_{X}\left(s, X_{t, x}^{\alpha}(s), \alpha_{s}\right) D \varphi\left(s, X_{t, x}^{\alpha}(s)\right), \alpha_{s}\right) .
$$

Let $\theta^{\alpha}=\theta_{1}^{\alpha} \wedge \theta_{2}^{\alpha}$, where

$$
\begin{gathered}
\theta_{1}^{\alpha}:=\inf \left\{s \in\left[\tau^{\alpha}, T\right]:\left(s, X_{t, x}^{\alpha}(s)\right) \notin B_{\varepsilon / 2}\left(t_{0}, x_{0}\right)\right\} \wedge T \text { and } \\
\theta_{2}^{\alpha}:=\inf \left\{s \in\left[\tau^{\alpha}, T\right]:\left|\bar{Y}^{\alpha}(s)-\varphi\left(s, X_{t, x}^{\alpha}(s)\right)\right| \geq \delta\right\} \wedge T .
\end{gathered}
$$

Set $\tilde{\mathfrak{u}}$ to be $\tilde{\mathfrak{u}}_{0}$ on $\left[\tau^{\alpha}, \theta^{\alpha}\right)$. Starting at $\theta^{\alpha}$, we will follow the strategy $\mathfrak{u}^{w,\left\{\theta^{\alpha}\right\}} \in$ $\mathfrak{U}\left(t,\left\{\theta^{\alpha}\right\}\right)$ which is "optimal" for $w$. In short,

$$
\tilde{\mathfrak{u}}[\alpha]=\left(\mathbb{1}_{A} \mathfrak{u}^{w,\left\{\tau^{\alpha}\right\}}[\alpha]+\mathbb{1}_{A^{c}}\left(\tilde{\mathfrak{u}}_{0}[\alpha] \mathbb{1}_{\left[t, \theta^{\alpha}\right)}+\mathfrak{u}^{w,\left\{\theta^{\alpha}\right\}}[\alpha] \mathbb{1}_{\left[\theta^{\alpha}, T\right]}\right)\right) \mathbb{1}_{\left[\tau^{\alpha}, T\right]}
$$

We note that $\tilde{\mathfrak{u}}_{0} \in \mathfrak{U}(t)$ by the pathwise uniqueness of $X$ 's, $Y$ 's and $\bar{Y}$ 's equations. Define $\mathfrak{u}^{*}[\alpha]:=\tilde{\mathfrak{u}}_{0}[\alpha] \mathbb{1}_{\left[t, \theta^{\alpha}\right)}+\mathfrak{u}^{w,\left\{\theta^{\alpha}\right\}}[\alpha] \mathbb{1}_{\left[\theta^{\alpha}, T\right]}$. From Lemma 5.2.11, $\mathfrak{u}^{*} \in \mathfrak{U}(t)$. By Definition 5.2.6, it is easy to see that $\tilde{\mathfrak{u}} \in \mathfrak{U}\left(t,\left\{\tau^{\alpha}\right\}\right)$ by the pathwise uniqueness of $X$ 's equation.

We construct $\boldsymbol{\rho}$ as follows. Let $\boldsymbol{\rho}^{w,\left\{\tau^{\alpha}\right\}} \in \mathbf{T}\left(t,\left\{\tau^{\alpha}\right\}\right)$ be the stopping strategy of the controller in Definition 5.4.1 for $w$ corresponding to $\left\{\tau^{\alpha}\right\}$ and $\boldsymbol{\rho}^{w,\left\{\theta^{\alpha}\right\}} \in \mathbf{T}\left(t,\left\{\theta^{\alpha}\right\}\right)$ be the stopping strategy in Definition 5.4.1 for $w$ corresponding to $\left\{\theta^{\alpha}\right\}$. Define

$$
\boldsymbol{\rho}[\alpha]=\mathbb{1}_{A} \boldsymbol{\rho}^{w,\left\{\tau^{\alpha}\right\}}[\alpha]+\mathbb{1}_{A^{c}} \boldsymbol{\rho}^{w,\left\{\theta^{\alpha}\right\}}[\alpha]
$$

Obviously, $\boldsymbol{\rho} \in \mathbf{T}\left(t,\left\{\tau^{\alpha}\right\}\right)$. To check that the constructions of $\tilde{\mathfrak{u}}$ and $\boldsymbol{\rho}$ works, it suffices to show that

$$
Y(\boldsymbol{\rho}[\alpha]) \leq g(X(\boldsymbol{\rho}[\alpha])) \quad \mathbb{P} \text {-a.s. on }\left\{Y\left(\tau^{\alpha}\right) \leq w\left(\tau^{\alpha}, X\left(\tau^{\alpha}\right)\right)\right\}
$$

where

$$
X:=X_{t, x}^{\alpha} \quad \text { and } \quad Y:=Y_{t, x, y}^{\mathfrak{u} \otimes_{\tau} \alpha \tilde{\mathfrak{u}}[\alpha], \alpha}
$$


We will carry out the proof in two steps:

(i) On the set $A \cap\left\{Y\left(\tau^{\alpha}\right) \leq w^{\kappa}\left(\tau^{\alpha}, X\left(\tau^{\alpha}\right)\right)\right\}$ : Note that

$$
A \cap\left\{Y\left(\tau^{\alpha}\right) \leq w^{\kappa}\left(\tau^{\alpha}, X\left(\tau^{\alpha}\right)\right)\right\} \subset\left\{Y\left(\tau^{\alpha}\right) \leq w\left(\tau^{\alpha}, X\left(\tau^{\alpha}\right)\right)\right\}
$$

Therefore, by the definitions of $\boldsymbol{\rho}$ and $\tilde{\mathfrak{u}}$ on $A$ and the fact $w \in \mathbb{U}_{b}^{-}$, we have

$$
Y(\boldsymbol{\rho}[\alpha]) \leq g(X(\boldsymbol{\rho}[\alpha])) \text { on } A \cap\left\{Y\left(\tau^{\alpha}\right) \leq w^{\kappa}\left(\tau^{\alpha}, X\left(\tau^{\alpha}\right)\right)\right\}
$$

(ii) On the set $A^{c} \cap\left\{Y\left(\tau^{\alpha}\right) \leq w^{\kappa}\left(\tau^{\alpha}, X\left(\tau^{\alpha}\right)\right\}\right.$ : Applying Itô's Formula, we get that

$$
\begin{gathered}
Y\left(\cdot \wedge \theta^{\alpha}\right)-\varphi\left(\cdot \wedge \theta^{\alpha}, X\left(\cdot \wedge \theta^{\alpha}\right)\right)=Y\left(\tau^{\alpha}\right)-\varphi\left(\tau^{\alpha}, X\left(\tau^{\alpha}\right)\right)+\int_{\tau^{\alpha}}^{\cdot \wedge \theta^{\alpha}} \gamma(s) d s, \text { where } \\
\gamma(s):=-\partial_{t} \varphi(s, X(s))+L^{\alpha_{s}}\left(s, X(s), Y(s), D \varphi(s, X(s)), D^{2} \varphi(s, X(s))\right) .
\end{gathered}
$$

From (5.4.5), $\gamma<0$ on $\left[\tau^{\alpha}, \theta^{\alpha}\right]$. This implies that $Y\left(\cdot \wedge \theta^{\alpha}\right)-\varphi\left(\cdot \wedge \theta^{\alpha}, X\left(\cdot \wedge \theta^{\alpha}\right)\right)$ is non-increasing on $\left[\tau^{\alpha}, T\right]$. Therefore,

$$
Y\left(\theta^{\alpha}\right)-\varphi\left(\theta^{\alpha}, X\left(\theta^{\alpha}\right)\right)-\kappa \leq Y\left(\tau^{\alpha}\right)-\varphi\left(\tau^{\alpha}, X\left(\tau^{\alpha}\right)\right)-\kappa \leq 0 .
$$

On the one hand, we get from (5.4.7) that

$$
0 \geq Y\left(\theta_{1}^{\alpha}\right)-\varphi\left(\theta_{1}^{\alpha}, X\left(\theta_{1}^{\alpha}\right)+\kappa\right) \geq Y\left(\theta_{1}^{\alpha}\right)-w\left(\theta_{1}^{\alpha}, X\left(\theta_{1}^{\alpha}\right)\right) \text { on }\left\{\theta_{1}^{\alpha}<\theta_{2}^{\alpha}\right\}
$$

On the other hand, due to (5.4.7) and the path continuity of $X$ and $Y$,

$$
Y\left(\theta_{2}^{\alpha}\right)-\varphi\left(\theta_{2}^{\alpha}, X\left(\theta_{2}^{\alpha}\right)\right)=-\delta \text { on }\left\{\theta_{1}^{\alpha} \geq \theta_{2}^{\alpha}\right\}
$$

Since $\varphi<w+\delta$ on $\bar{B}_{\varepsilon / 2}\left(t_{0}, x_{0}\right)$

$$
Y\left(\theta_{2}^{\alpha}\right)-w\left(\theta_{2}^{\alpha}, X\left(\theta_{2}^{\alpha}\right)\right)=\varphi\left(\theta_{2}^{\alpha}, X\left(\theta_{2}^{\alpha}\right)\right)-w\left(\theta_{2}^{\alpha}, X\left(\theta_{2}^{\alpha}\right)\right)-\delta<0 \text { on }\left\{\theta_{1}^{\alpha} \geq \theta_{2}^{\alpha}\right\}
$$

Combining (5.4.8) and (5.4.9), we obtain

$$
Y\left(\theta^{\alpha}\right)-w\left(\theta^{\alpha}, X\left(\theta^{\alpha}\right)\right) \leq 0 \text { on } A^{c} \cap\left\{Y\left(\tau^{\alpha}\right) \leq w^{\kappa}\left(\tau^{\alpha}, X^{\alpha}\right)\right\}
$$


This equation, together with the facts that $\boldsymbol{\rho}=\boldsymbol{\rho}^{w, \theta^{\alpha}}$ and $\tilde{\mathfrak{u}}=\mathfrak{u}^{w,\left\{\theta^{\alpha}\right\}}$ on $A^{c}$, implies that

$$
Y(\boldsymbol{\rho}[\alpha]) \leq g(X(\boldsymbol{\rho}[\alpha])) \text { on } A^{c} \cap\left\{Y\left(\tau^{\alpha}\right) \leq w^{\kappa}\left(\tau^{\alpha}, X^{\alpha}\right)\right\}
$$

\section{Step 2. ( $V_{b}^{+}$is a viscosity sub-solution)}

2.1 The interior super-solution property: Assume, on the contrary, that $\left(t_{0}, x_{0}\right) \in \mathbb{D}_{i}$ and $\varphi \in C^{1,2}(\mathbb{D})$ are such that

$$
0=V_{b}^{+}\left(t_{0}, x_{0}\right)-\varphi\left(t_{0}, x_{0}\right)=\max _{(t, x) \in \mathbb{D}_{i}}\left(V_{b}^{+}(t, x)-\varphi(t, x)\right)
$$

$V_{b}^{+}\left(t_{0}, x_{0}\right)>g\left(x_{0}\right)$ and $-\partial_{t} \varphi\left(t_{0}, x_{0}\right)+F \varphi\left(t_{0}, x_{0}\right)>0$. Then there exists $a_{0} \in A$ such that

$$
-\partial_{t} \varphi\left(t_{0}, x_{0}\right)+L^{u_{0}, a_{0}} \varphi\left(t_{0}, x_{0}\right)>0
$$

where $u_{0}=\hat{u}\left(t_{0}, x_{0}, \varphi\left(t_{0}, x_{0}\right), \sigma_{X}\left(t_{0}, x_{0}, a_{0}\right) D \varphi\left(t_{0}, x_{0}\right), a_{0}\right)$. From the continuity assumption on the coefficients, the upper semi-continuity of $g$ and the continuity of $\hat{u}$ in Assumption 5.2.12, there exist $\varepsilon>0$ and $\delta>0$ such that

$$
\varphi(t, x)>g(x)+\varepsilon \text { for all }(t, x) \in \bar{B}_{\varepsilon}\left(t_{0}, x_{0}\right)
$$

and

$$
\begin{gathered}
-\partial_{t} \varphi(t, x)+L^{u, a_{0}}\left(t, x, y, D \varphi, D^{2} \varphi\right)>0,(t, x, y, u) \in \bar{B}_{\varepsilon}\left(t_{0}, x_{0}\right) \times \mathbb{R} \times U \\
\text { s.t. }|y-\varphi(t, x)| \leq \delta \text { and }\left|\sigma_{Y}\left(t, x, y, u, a_{0}\right)-\sigma_{X}\left(t, x, a_{0}\right) D \varphi(t, x)\right| \leq \delta .
\end{gathered}
$$

Since $\varphi>V_{b}^{+}$on the compact set $\mathbb{T}:=\bar{B}_{\varepsilon}\left(t_{0}, x_{0}\right)-B_{\varepsilon / 2}\left(t_{0}, x_{0}\right)$ and $V_{b}^{+}$is USC, $\varphi>V_{b}^{+}+2 \eta$ on $\mathbb{T}$ for some $\eta>0$. Let $\left\{w_{n}\right\}$ be a sequence in $\mathbb{U}_{b}^{+}$such that $w_{n} \searrow V_{b}^{+}$. A Dini type argument shows that for large enough $n$,

$$
\varphi>w_{n}+\eta \text { on } \mathbb{T}, \varphi>w_{n}-\delta \text { on } \bar{B}_{\varepsilon / 2}\left(t_{0}, x_{0}\right)
$$


Fix such an $n$ and let $w=w_{n}$. For $\kappa \in(0, \eta \wedge \delta \wedge \varepsilon)$, define

$$
w^{\kappa}:=\left\{\begin{array}{l}
(\varphi-\kappa) \wedge w \text { on } \bar{B}_{\varepsilon}\left(t_{0}, x_{0}\right), \\
w \text { outside } \bar{B}_{\varepsilon}\left(t_{0}, x_{0}\right) .
\end{array}\right.
$$

Since $w^{\kappa}\left(t_{0}, x_{0}\right)=\varphi\left(t_{0}, x_{0}\right)+\kappa<V_{b}^{+}\left(t_{0}, x_{0}\right)$, we will obtain a contradiction if we can show $w^{\kappa} \in \mathbb{U}_{b}^{+}$. Obviously, $w^{\kappa}$ is continuous and bounded. By (5.4.11) and $w \in \mathbb{U}_{b}^{+}$, $w^{\kappa}(t, x) \geq g(x)$ for all $(t, x) \in \mathbb{D}$.

$\operatorname{Fix}(t, x, y) \in \mathbb{D}_{i} \times \mathbb{R},\left\{\tau^{\alpha}\right\} \in \mathbb{T}_{t}, \mathfrak{u} \in \mathfrak{U}(t), \boldsymbol{\rho} \in \mathbf{T}\left(t,\left\{\tau^{\alpha}\right\}\right)$ and $\alpha \in \mathcal{A}^{t}$. we will construct an "optimal" control $\widetilde{\alpha} \in \mathcal{A}^{t}$ in Definition 5.4.2 for $w^{\kappa}$. Let $A=$ $\left\{w\left(\tau^{\alpha}, X_{t, x}^{\alpha}\left(\tau^{\alpha}\right)\right)=w^{\kappa}\left(\tau^{\alpha}, X_{t, x}^{\alpha}\left(\tau^{\alpha}\right)\right)\right\}$. We divide the construction into two cases:

(i) On $A$ : Since $w \in \mathbb{U}_{b}^{+}$, there exists $\widetilde{\alpha}^{w, \tau^{\alpha}}$ which is "optimal" for Nature in the sense of Definition 5.4.2 given $\mathfrak{u},\left\{\tau^{\alpha}\right\}, \boldsymbol{\rho}$ and $\alpha$. Set $\widetilde{\alpha}$ to be $\widetilde{\alpha}^{w, \tau^{\alpha}}$ on $A$.

(ii) On $A^{c}$ : Let $\theta^{\alpha}=\theta_{1}^{\alpha} \wedge \theta_{2}^{\alpha}$, where

$$
\begin{gathered}
\theta_{1}^{\alpha}:=\inf \left\{s \in\left[\tau^{\alpha}, T\right]:\left(s, X_{t, x}^{\alpha \otimes_{\tau^{\alpha} a_{0}}}(s)\right) \notin B\left(t_{0}, x_{0}, \varepsilon / 2\right)\right\} \wedge T \text { and } \\
\theta_{2}^{\alpha}:=\inf \left\{s \in\left[\tau^{\alpha}, T\right]:\left|Y_{t, x, y}^{\mathfrak{u}, \alpha \otimes_{\tau^{\alpha} a_{0}}}(s)-\varphi\left(s, X_{t, x}^{\alpha \otimes_{\tau^{\alpha} a_{0}}}(s)\right)\right| \geq \delta\right\} \wedge T .
\end{gathered}
$$

Set $\widetilde{\alpha}=a_{0}$ on $\left[\tau^{\alpha}, \theta^{\alpha}\right)$. Starting from $\theta^{\alpha}$, choose $\widetilde{\alpha}=\widetilde{\alpha}^{w, \theta^{\alpha}}$, where the latter is "optimal" for Nature given $\mathfrak{u},\left\{\tau^{\alpha}\right\}, \boldsymbol{\rho}$ and $\alpha$ this time onward. In short,

$$
\widetilde{\alpha}=\left(\mathbb{1}_{A} \widetilde{\alpha}^{w, \tau^{\alpha}}+\mathbb{1}_{A^{c}}\left(a_{0} \mathbb{1}_{\left[t, \theta^{\alpha}\right)}+\widetilde{\alpha}^{w, \theta^{\alpha}} \mathbb{1}_{\left[\theta^{\alpha}, T\right]}\right)\right) \mathbb{1}_{\left[\tau^{\alpha}, T\right]}
$$

Let us check the construction above works. Set $(X, Y):=\left(X_{t, x}^{\alpha \otimes_{\tau^{\alpha}} \widetilde{\alpha}}, Y_{t, x, y}^{\mathfrak{u}, \alpha \otimes_{\tau} \alpha \widetilde{\alpha}}\right)$. Note that

$$
\begin{aligned}
& X(s)=\mathbb{1}_{A} X_{t, x}^{\alpha \otimes_{\tau^{\alpha}} \widetilde{\alpha}^{w, \tau^{\alpha}}}(s)+\mathbb{1}_{A^{c}} X_{t, x}^{\alpha \otimes_{\tau^{\alpha} a_{0}}}(s) \quad \text { for } \tau^{\alpha} \leq s \leq \theta^{\alpha}, \\
& Y(s)=\mathbb{1}_{A} Y_{t, x, y}^{\mathfrak{u}, \alpha \otimes_{\tau^{\alpha}} \widetilde{\alpha}^{w, \tau^{\alpha}}}(s)+\mathbb{1}_{A^{c}} Y_{t, x, y}^{\mathfrak{u}, \alpha \otimes_{\tau^{\alpha} a_{0}}}(s) \quad \text { for } \tau^{\alpha} \leq s \leq \theta^{\alpha} .
\end{aligned}
$$

Let

$$
\begin{gathered}
E=\left\{Y\left(\tau^{\alpha}\right)>w^{\kappa}\left(\tau^{\alpha}, X\left(\tau^{\alpha}\right)\right)\right\}, \quad E_{0}=E \cap A, \quad E_{1}=E \cap A^{c}, \\
G=\left\{Y(\boldsymbol{\rho}[\alpha])>w^{\kappa}(\boldsymbol{\rho}[\alpha], X(\boldsymbol{\rho}[\alpha])\}, \quad G_{0}=\{Y(\boldsymbol{\rho}[\alpha])>w(\boldsymbol{\rho}[\alpha], X(\boldsymbol{\rho}[\alpha])\} .\right.
\end{gathered}
$$


Observe that $E=E_{0} \cup E_{1}, E_{0} \cap E_{1}=\emptyset$ and $G_{0} \subset G$. We will show in the rest of the step that $P(G \mid B)>0$ for any $\mathcal{F}_{\tau^{\alpha}}^{t}$-measurable, non-null set $B \subset E$. In fact, it suffices to show that $\mathbb{P}(G \cap B)>0$. Noting that $\mathbb{P}(G \cap B)=\mathbb{P}\left(G \cap B \cap E_{0}\right)+\mathbb{P}\left(G \cap B \cap E_{1}\right)$ and $\mathbb{P}(B)=\mathbb{P}\left(B \cap E_{0}\right)+\mathbb{P}\left(B \cap E_{1}\right)$, we divide the proof into two steps which will yield the desired result.

(i) If $\mathbb{P}\left(B \cap E_{0}\right)>0$ : Since $w \in \mathbb{U}_{b}^{-}, B \cap E_{0} \subset\left\{Y\left(\tau^{\alpha}\right)>w\left(\tau^{\alpha}, X\left(\tau^{\alpha}\right)\right)\right\}$ and $B \cap E_{0} \subset A$, it follows from the definition of $\widetilde{\alpha}^{w, \tau^{\alpha}}$ that

$$
\mathbb{P}\left(G_{0} \mid B \cap E_{0}\right)=\mathbb{P}\left(Y_{t, x, y}^{\mathfrak{u}, \alpha \otimes_{\tau^{\alpha}} \widetilde{\alpha}^{w, \tau^{\alpha}}}(\boldsymbol{\rho}[\alpha])>w\left(\boldsymbol{\rho}[\alpha], X_{t, x}^{\alpha \otimes_{\tau^{\alpha}} \widetilde{\alpha}^{w, \tau^{\alpha}}}(\boldsymbol{\rho}[\alpha])\right) \mid B \cap E_{0}\right)>0 .
$$

This further implies that $\mathbb{P}\left(G \cap B \cap E_{0}\right) \geq \mathbb{P}\left(G_{0} \cap B \cap E_{0}\right)>0$.

(ii) If $\mathbb{P}\left(B \cap E_{1}\right)>0$ : From (5.4.13) and $B \cap E_{1} \subset A^{c}$,

$\mathbb{P}\left(Y\left(\theta^{\alpha}\right)>w^{\kappa}\left(\theta^{\alpha}, X\left(\theta^{\alpha}\right)\right) \mid B \cap E_{1}\right)=\mathbb{P}\left(Y_{t, x, y}^{\mathfrak{u}, \alpha \otimes_{\tau} \alpha a_{0}}\left(\theta^{\alpha}\right)>w^{\kappa}\left(\theta^{\alpha}, X_{t, x}^{\alpha \otimes_{\tau} \alpha a_{0}}\left(\theta^{\alpha}\right)\right) \mid B \cap E_{1}\right)$.

The analysis in [6] shows that

$$
\Delta(s)=Y\left(s \wedge \theta^{\alpha}\right)-\varphi\left(s \wedge \theta^{\alpha}, X\left(s \wedge \theta^{\alpha}\right)\right)+\kappa .
$$

is a super-martingale up to a change of measure. We will summarize these arguments here. Let

$$
\begin{aligned}
& \lambda(s):=\sigma_{Y}\left(s, X(s), Y(s), \mathfrak{u}\left[a_{0}\right]_{s}, a_{0}\right)-\sigma_{X}\left(s, X(s), a_{0}\right) D \varphi(s, X(s)), \\
& \beta(s):=L^{\mathfrak{u}\left[a_{0}\right]_{s}, a_{0}}\left(s, X(s), Y(s), D \varphi(s, X(s)), D^{2} \varphi(s, X(s))\right)|\lambda(s)|^{-2} \lambda(s) \mathbb{1}_{\{|\lambda(s)|>\delta\}} .
\end{aligned}
$$

From the definition of $\theta^{\alpha}$ and the regularity and growth conditions in Assumptions 5.2.1 and 5.2.14, we can check that $\beta$ is uniformly bounded on $\left[\tau^{\alpha}, \theta^{\alpha}\right]$. This ensures that the positive exponential local martingale $M$ defined by the SDE

$$
M(\cdot)=1+\int_{\tau^{\alpha}}^{\cdot \wedge \theta^{\alpha}} M(s) \beta_{s}^{\top} d W_{s}
$$


is a true martingale after $\tau^{\alpha}$. From (5.4.12), an application of Itô's formula immediately implies that $M \Delta$ is a local sub-martingale. By similar arguments to those in Step 2.1 of Theorem 4.3.4's proof, we further know that $M \Delta$ is a sub-martingale.

Since $\Delta\left(\tau^{\alpha}\right)>0$ on $B \cap E_{1}$, it follows from the sub-martingale property of $M \Delta$ that there exists a non-null $K \subset B \cap E_{1}, K \in \mathcal{F}_{\tau^{\alpha}}^{t}$ such that $\Delta\left(\theta^{\alpha} \wedge \boldsymbol{\rho}[\alpha]\right)>0$ on $K$. From the decomposition

$$
\begin{aligned}
\Delta\left(\theta^{\alpha} \wedge \boldsymbol{\rho}[\alpha]\right) \mathbb{1}_{K} & =\left(Y\left(\theta_{1}^{\alpha}\right)-\varphi\left(\theta_{1}^{\alpha}, X\left(\theta_{1}^{\alpha}\right)\right)+\kappa\right) \mathbb{1}_{K \cap\left\{\theta_{1}^{\alpha}<\theta_{2}^{\alpha} \wedge \boldsymbol{\rho}[\alpha]\right\}} \\
& +\left(Y\left(\theta_{2}^{\alpha}\right)-\varphi\left(\theta_{2}^{\alpha}, X\left(\theta_{2}^{\alpha}\right)\right)+\kappa\right) \mathbb{1}_{K \cap\left\{\theta_{2}^{\alpha} \leq \theta_{1}^{\alpha} \wedge \boldsymbol{\rho}[\alpha]\right\}} \\
& +(Y(\boldsymbol{\rho}[\alpha])-\varphi(\boldsymbol{\rho}[\alpha], X(\boldsymbol{\rho}[\alpha]))+\kappa) \mathbb{1}_{K \cap\left\{\boldsymbol{\rho}[\alpha]<\theta^{\alpha}\right\}}
\end{aligned}
$$

we get that

$$
\begin{array}{lc}
Y\left(\theta_{1}^{\alpha}\right)-\varphi\left(\theta_{1}^{\alpha}, X\left(\theta_{1}^{\alpha}\right)\right)+\kappa>0 \quad \text { on } \quad K \cap\left\{\theta_{1}^{\alpha}<\theta_{2}^{\alpha} \wedge \boldsymbol{\rho}[\alpha]\right\}, \\
Y\left(\theta_{2}^{\alpha}\right)-\varphi\left(\theta_{2}^{\alpha}, X\left(\theta_{2}^{\alpha}\right)\right)+\kappa>0 \quad \text { on } \quad K \cap\left\{\theta_{2}^{\alpha} \leq \theta_{1}^{\alpha} \wedge \boldsymbol{\rho}[\alpha]\right\}, \\
Y(\boldsymbol{\rho}[\alpha])-\varphi\left(\boldsymbol{\rho}[\alpha], X(\boldsymbol{\rho}[\alpha])+\kappa>0 \quad \text { on } \quad K \cap\left\{\boldsymbol{\rho}[\alpha]<\theta^{\alpha}\right\} .\right.
\end{array}
$$

On the one hand,

$$
\varphi\left(\theta_{1}^{\alpha}, X\left(\theta_{1}^{\alpha}\right)\right)-\kappa>w\left(\theta_{1}^{\alpha}, X\left(\theta_{1}^{\alpha}\right)\right) \text { on } K \cap\left\{\theta_{1}^{\alpha}<\theta_{2}^{\alpha} \wedge \boldsymbol{\rho}[\alpha]\right\}
$$

Then from (5.4.14), the equation above yields that

$$
Y\left(\theta_{1}^{\alpha}\right)>w\left(\theta_{1}^{\alpha}, X\left(\theta_{1}^{\alpha}\right)\right) \quad \text { on } \quad K \cap\left\{\theta_{1}^{\alpha}<\theta_{2}^{\alpha} \wedge \boldsymbol{\rho}[\alpha]\right\}
$$

On the other hand,

$$
Y\left(\theta_{2}^{\alpha}\right)-\varphi\left(\theta_{2}^{\alpha}, X\left(\theta_{2}^{\alpha}\right)\right)=\delta . \text { on } K \cap\left\{\theta_{2}^{\alpha} \leq \theta_{1}^{\alpha} \wedge \rho[\alpha]\right\}
$$

The right-hand-side can not be $-\delta$, otherwise (5.4.15) would be contradicted. Recalling that $\varphi>w-\delta$ on $\bar{B}_{\varepsilon / 2}\left(t_{0}, x_{0}\right)$, this observation gives that

$$
Y\left(\theta_{2}^{\alpha}\right)=\varphi\left(\theta_{2}^{\alpha}, X\left(\theta_{2}^{\alpha}\right)\right)+\delta>w\left(\theta_{2}^{\alpha}, X\left(\theta_{2}^{\alpha}\right)\right) \quad \text { on } \quad K \cap\left\{\theta_{2}^{\alpha} \leq \theta_{1}^{\alpha} \wedge \boldsymbol{\rho}[\alpha]\right\}
$$


Therefore, we obtain in (5.4.17) and (5.4.18) that

$$
Y\left(\theta^{\alpha}\right)>w\left(\theta^{\alpha}, X\left(\theta^{\alpha}\right)\right) \quad \text { on } \quad K \cap\left\{\theta^{\alpha} \leq \boldsymbol{\rho}[\alpha]\right\}
$$

By the definition of stochastic sub-solutions and of $\widetilde{\alpha}^{w, \theta^{\alpha}}$, we have that

$$
\mathbb{P}\left(G_{0} \mid K \cap\left\{\theta^{\alpha} \leq \boldsymbol{\rho}[\alpha]\right\}\right)>0 \quad \text { if } \quad \mathbb{P}\left(K \cap\left\{\theta^{\alpha} \leq \boldsymbol{\rho}[\alpha]\right\}\right)>0
$$

Also, (5.4.16) implies that

$$
\mathbb{P}\left(G \mid K \cap\left\{\theta^{\alpha}>\boldsymbol{\rho}[\alpha]\right\}\right)>0 \quad \text { if } \quad \mathbb{P}\left(K \cap\left\{\theta^{\alpha}>\boldsymbol{\rho}[\alpha]\right\}\right)>0 .
$$

Since $\mathbb{P}(K)>0, G_{0} \subset G$, and $K \subset E_{1} \cap B$, (5.4.19) and (5.4.20) imply

$$
\mathbb{P}\left(G \cap E_{1} \cap B\right)>0 .
$$

2.2 The boundary condition: The boundary condition can be proven by following a similar proof to Step 2.2 in Theorem 4.3.4.

To characterize $V_{b}$ as the unique viscosity solution of (5.2.3), we need a comparison principle.

Proposition 5.4.8. Under Assumption 5.2.1, 5.2.12 and 5.2.13, the comparison principle for (5.2.3) holds. More precisely, if U (resp. W) is a bounded LSC viscosity sub-solution (resp. a bounded USC viscosity super-solution) to (5.2.3) and $U \leq W$ on $\mathbb{D}_{T}$, then $U \leq W$ on $\mathbb{D}$.

Proof. The proposition follows from the arguments in Proposition 4.3.5.

Corollary 5.4.9. Under Assumption 5.2.1, 5.2.12, 5.2.14 and 5.4.4, $V_{b}$ is the unique bounded continuous viscosity solution of (5.2.3).

Proof. This corollary follows from the same arguments in Corollary 4.3.6. 


\subsection{Comparison of $V_{s}$ and $V_{b}$}

Proposition 5.5.1. Let Assumptions 5.2.1, 5.2.12, 5.2.13, 5.2.14 and 5.4.4 hold. Then

1. $V_{s} \geq V_{b}$ on $\mathbb{D}$.

2. If $A=a_{0}$ for some $a_{0}, V_{s}=V_{b}$.

Proof. The second claim is trivial, by the uniqueness of the viscosity solutions to (5.2.2) and the fact that $H=F$ if $A$ is a singleton. The proof of the first claim is similar to that of Proposition 4.3.5. We will only outline the key steps of the proof. Step 1. Without loss of generality, we assume that

$$
\exists \gamma>0 \text { such that } H(t, x, y, p, M)-H\left(t, x, y^{\prime}, p, M\right)>\gamma\left(y-y^{\prime}\right)
$$

for all $y>y^{\prime}$. Otherwise, we could follow the arguments in Step 1 of Proposition 4.3.5.

Step 2. We know from Theorem 5.3.7 that $V_{s}$ is the unique bounded continuous viscosity solution of (5.2.2). We claim that for large enough $\lambda>0, V_{s}^{\delta}$ is a continuous viscosity super-solution of (5.2.2) for any $\delta>0$, where $V_{s}^{\delta}(t, x):=V_{s}(t, x)+\delta e^{-\lambda t}(1+$ $\left.|x|^{2}\right)$ for $(t, x) \in \mathbb{D}$. Such a claim is proved in Step 2 of Proposition 4.3.5.

Step 3. In this step, we show that $V_{b} \leq V_{s}^{\delta}$ on $\mathbb{D}$ for all $\delta>0$. Then by taking $\delta \rightarrow 0$, we get that $V_{b} \leq V_{s}$ on $\mathbb{D}$. The proof of this step follows from a very similar arguments in Step 3 of Proposition 4.3.5.

Remark 5.5.2. As we mentioned, $V_{s}$ and $V_{b}$ are interpreted as hedging prices of American options with model uncertainty. Although they don't compare by definition, the above proposition show that $V_{s} \geq V_{b}$ on $\mathbb{D}$ without proving any duality results. For discussion about hedging under model uncertainty, we refer the readers to [5] and the references therein. 
BIBLIOGRAPHY 


\section{BIBLIOGRAPHY}

[1] Guy Barles and Cyril Imbert. Second-order elliptic integro-differential equations: viscosity solutions' theory revisited. Ann. Inst. H. Poincaré Anal. Non Linéaire, 25(3):567-585, 2008.

[2] E. Bayraktar and Y. Zhang. Stochastic perron's method for the probability of lifetime ruin problem under transaction costs. SIAM Journal on Control and Optimization, 53(1):91-113, 2015.

[3] Erhan Bayraktar, Andrea Cosso, and Huyen Pham. Robust feedback switching control: dynamic programming and viscosity solutions. to appear on SIAM Journal on Control and Optimization., June 2016.

[4] Erhan Bayraktar and Yu-Jui Huang. On the multidimensional controller-and-stopper games. SIAM J. Control Optim., 51(2):1263-1297, 2013.

[5] Erhan Bayraktar, Yu-Jui Huang, and Zhou Zhou. On hedging American options under model uncertainty. SIAM J. Financial Math., 6(1):425-447, 2015.

[6] Erhan Bayraktar and Jiaqi Li. Stochastic Perron for stochastic target games. Ann. Appl. Probab., 26(2):1082-1110, 2016.

[7] Erhan Bayraktar and Jiaqi Li. Stochastic perron for stochastic target problems. Journal of Optimization Theory and Applications, 170(3):1026-1054, 2016.

[8] Erhan Bayraktar and Mihai Sîrbu. Stochastic Perron's method and verification without smoothness using viscosity comparison: the linear case. Proc. Amer. Math. Soc., 140(10):3645$3654,2012$.

[9] Erhan Bayraktar and Mihai Sîrbu. Stochastic Perron's method for Hamilton-Jacobi-Bellman equations. SIAM J. Control Optim., 51(6):4274-4294, 2013.

[10] Erhan Bayraktar and Mihai Sîrbu. Stochastic Perron's method and verification without smoothness using viscosity comparison: obstacle problems and Dynkin games. Proc. Amer. Math. Soc., 142(4):1399-1412, 2014.

[11] Erhan Bayraktar and Virginia R. Young. Proving regularity of the minimal probability of ruin via a game of stopping and control. Finance Stoch., 15(4):785-818, 2011.

[12] Bruno Bouchard. Stochastic targets with mixed diffusion processes and viscosity solutions. Stochastic Process. Appl., 101(2):273-302, 2002.

[13] Bruno Bouchard and Ngoc Minh Dang. Optimal control versus stochastic target problems: an equivalence result. Systems Control Lett., 61(2):343-346, 2012.

[14] Bruno Bouchard, Romuald Elie, and Nizar Touzi. Stochastic target problems with controlled loss. SIAM J. Control Optim., 48(5):3123-3150, 2009/10.

[15] Bruno Bouchard, Ludovic Moreau, and Marcel Nutz. Stochastic target games with controlled loss. Ann. Appl. Probab., 24(3):899-934, 2014. 
[16] Bruno Bouchard and Marcel Nutz. Stochastic target games and dynamic programming via regularized viscosity solutions. Mathematics of Operations Research, 41(1):109-124, 2016.

[17] Rainer Buckdahn and Juan Li. Probabilistic interpretation for systems of Isaacs equations with two reflecting barriers. NoDEA Nonlinear Differential Equations Appl., 16(3):381-420, 2009 .

[18] Michael G. Crandall, Hitoshi Ishii, and Pierre-Louis Lions. User's guide to viscosity solutions of second order partial differential equations. Bull. Amer. Math. Soc. (N.S.), 27(1):1-67, 1992.

[19] Ioannis Karatzas and S. G. Kou. Hedging American contingent claims with constrained portfolios. Finance Stoch., 2(3):215-258, 1998.

[20] Ioannis Karatzas and Steven E. Shreve. Brownian motion and stochastic calculus, volume 113 of Graduate Texts in Mathematics. Springer-Verlag, New York, second edition, 1991.

[21] Ioannis Karatzas and William D. Sudderth. Control and stopping of a diffusion process on an interval. Ann. Appl. Probab., 9(1):188-196, 1999.

[22] Ioannis Karatzas and William D. Sudderth. The controller-and-stopper game for a linear diffusion. Ann. Probab., 29(3):1111-1127, 2001.

[23] Ioannis Karatzas and Ingrid-Mona Zamfirescu. Martingale approach to stochastic control with discretionary stopping. Appl. Math. Optim., 53(2):163-184, 2006.

[24] Ioannis Karatzas and Ingrid-Mona Zamfirescu. Martingale approach to stochastic differential games of control and stopping. Ann. Probab., 36(4):1495-1527, 2008.

[25] Ludovic Moreau. Stochastic target problems with controlled loss in jump diffusion models. SIAM Journal on Control and Optimization, 49(6):2577-2607, 2011.

[26] Huyên Pham. Continuous-time stochastic control and optimization with financial applications, volume 61 of Stochastic Modelling and Applied Probability. Springer-Verlag, Berlin, 2009.

[27] D. B. Rokhlin. Stochastic perron's method for optimal control problems with state constraints. Electron. Commun. Probab., 19:no. 73, 1-15, 2014.

[28] D. B. Rokhlin. Verification by stochastic Perron's method in stochastic exit time control problems. J. Math. Anal. Appl., 419(1):433-446, 2014.

[29] Mihai Sîrbu. A note on the strong formulation of stochastic control problems with model uncertainty. Electron. Commun. Probab., 19:no. 81, 10, 2014.

[30] Mihai Sîrbu. Stochastic Perron's method and elementary strategies for zero-sum differential games. SIAM J. Control Optim., 52(3):1693-1711, 2014.

[31] H. Mete Soner and Nizar Touzi. Superreplication under gamma constraints. SIAM J. Control Optim., 39(1):73-96, 2000.

[32] H. Mete Soner and Nizar Touzi. Dynamic programming for stochastic target problems and geometric flows. J. Eur. Math. Soc. (JEMS), 4(3):201-236, 2002.

[33] H. Mete Soner and Nizar Touzi. Stochastic target problems, dynamic programming, and viscosity solutions. SIAM J. Control Optim., 41(2):404-424, 2002.

[34] Nizar Touzi. Optimal stochastic control, stochastic target problems, and backward SDE, volume 29 of Fields Institute Monographs. Springer, New York; Fields Institute for Research in Mathematical Sciences, Toronto, ON, 2013. With Chapter 13 by Angès Tourin. 